\title{
Production of bioengineered outer membrane vesicles as vaccine platform
}

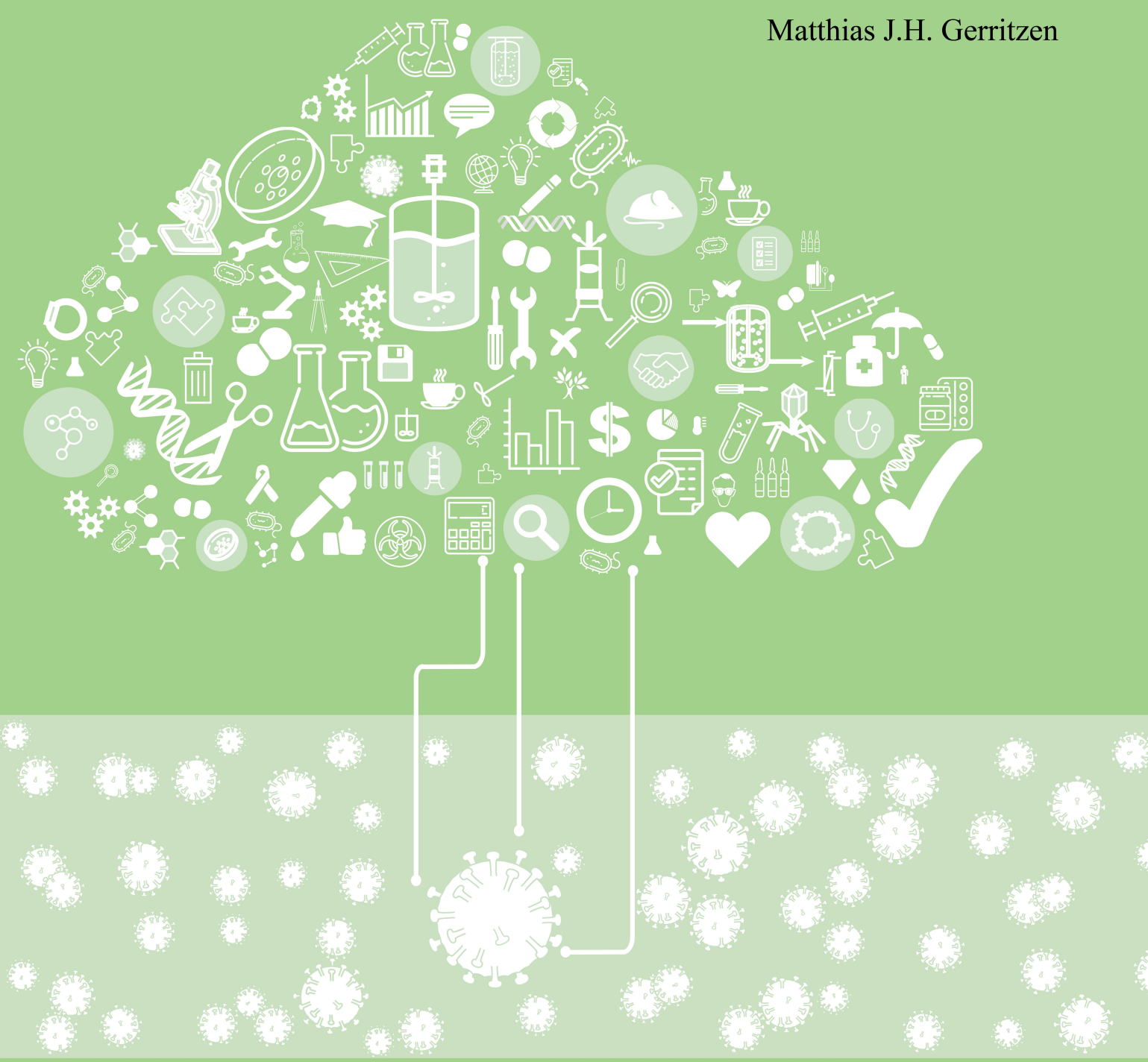




\section{Propositions}

1 Neisseria meningitidis outer membrane vesicle release is triggered by oxidative stress (this thesis)

2 Spontaneous released Neisseria meningitidis outer membrane vesicles are an ideal basis for a vaccine platform (this thesis)

3 A central vaccine control authority should be introduced to prevent fake vaccines entering the market

4 Connecting acid and base to their corresponding bioreactor pumps is an often-overlooked critical process parameter

5 Research and development laboratories must embrace singleuse materials to reduce their carbon footprint

$6 \quad$ Vaccination should be voluntary

Propositions belonging to the thesis, entitled

"Production of bioengineered outer membrane vesicles as a vaccine platform"

Matthias J.H. Gerritzen

Wageningen, 9 April 2019 
Production of bioengineered outer membrane vesicles as a vaccine platform

Matthias J.H. Gerritzen 


\section{Thesis committee}

\section{Promotor}

Prof. Dr R.H. Wijffels

Professor of Bioprocess Engineering

Wageningen University \& Research

\section{Co-promotor}

Dr M. Stork

Head of Process Development Bacterial Vaccines

Institute for Translation Vaccinology (Intravacc), Bilthoven

Dr D.E. Martens

Associate professor of Bioprocess Engineering

Wageningen University \& Research

\section{other members}

Prof. Dr J. Van der Oost, Wageningen University \& Research

Prof. Dr M.P. DeLisa, Cornell University, Ithaca, USA

Dr J. Luirink, VU University Amsterdam

Dr M. Streefland, Kiadis Pharma, Amsterdam

This research was conducted under the auspices of the Graduate School of Advanced Studies in Food Technology, Agrobiotechnology, Nutrition and Health Sciences (VLAG) 


\title{
Production of bioengineered outer membrane vesicles as a vaccine platform
}

\author{
Matthias J.H. Gerritzen
}

Thesis

submitted in fulfillment of the requirements for the degree of doctor at Wageningen University by the authority of the Rector Magnificus

Prof. Dr A.P.J. Mol,

in the presence of the

Thesis Committee appointed by the Academic Board

to be defended in public

on Tuesday 9 April 2019

at 1:30 p.m. in the Aula. 
Matthias J.H. Gerritzen

Production of bioengineered outer membrane vesicles as a vaccine platform 174 pages

PhD thesis, Wageningen University, Wageningen, The Netherlands (2019)

With references and summary

ISBN 978-94-6343-429-4

DOI https://doi.org/10.18174/471396 


\section{Table of contents}

$\begin{array}{ll}\text { Chapter } 1 & 7\end{array}$

Introduction and thesis outline

Chapter 2

Bioengineering bacterial outer membrane vesicles as vaccine platform.

\section{Chapter 3}

High throughput nanoparticle tracking analysis for monitoring outer

membrane vesicle production

\section{Chapter 4}

Sulfate depletion triggers overproduction of phospholipids and the release of outer membrane vesicles by Neisseria meningitidis

\section{Chapter 5}

High dissolved oxygen tension triggers outer membrane vesicle formation by Neisseria meningitidis

\section{Chapter 6}

Spontaneously released Neisseria meningitidis outer membrane vesicles as vaccine platform

\section{Chapter 7}

Continuous production of Neisseria meningitidis outer membrane vesicles

\section{Chapter 8}

General discussion

\section{Addendum}

Summary

Dankwoord

Curriculum vitae

List of publications 

Chapter 1

Introduction and thesis outline 


\section{Vaccines}

Vaccination is a proven method of preventing infectious diseases [1]. Through the administration of antigenic material, vaccines stimulate an individual's immune system and thereby provide protection against subsequent infection. Vaccines that are used to protect healthy individuals against disease in the form of a preventive intervention are called prophylactic vaccines. Prophylactic vaccination has resulted in the eradication of smallpox and rinderpest [2,3] and the eradication of poliomyelitis lays in sight [4]. Because of these successes, there is an increasing demand for new prophylactic vaccines. This increase is due to a variety of reasons. Firstly, infectious diseases show antigenic variation resulting in evasion of the immune responses elicited by existing vaccines, thereby reducing their potency [5]. Secondly, it is becoming increasingly difficult to treat bacterial disease by antibiotics as more bacteria arise that acquired antimicrobial resistance [6]. Vaccination would be an effective method to prevent bacterial infections that are increasingly difficult to treat with antibiotics due to antimicrobial resistance $[7,8]$. Thirdly, the introduction of single serogroup-specific vaccines for a disease can cause another serogroup to emerge [9]. Lastly, prophylactic vaccine development is needed for diseases that were previously not recognized as infectious diseases, such as cervical cancer, which is linked to Human Papiloma Virus, and Type I diabetes, which is linked to Coxsackievirus $[10,11]$.

Besides the growing need for the development of prophylactic vaccines to prevent infectious disease, there is increasing attention for the development of therapeutic vaccines. In contrast to prophylactic vaccines, therapeutic vaccines are used as immunotherapy to treat existing disease. Therapeutic vaccines stimulate the immune system such that the cause of the disease is countered by the immune system. Therapeutic vaccines can be personalized and show a huge potential in for example cancer immunotherapy [12].

\section{Vaccine development and vaccine platforms}

The development of traditional prophylactic vaccines is based on the inactivation or attenuation of the infectious agent. Although proven successful in preventing infectious disease, these traditional methods have drawbacks. Live attenuated vaccines are based on weakened pathogens that could revert to a pathogenic form. Although very rare, this may lead to serious adverse effects. Inactivated vaccines do not hold this risk, but 
require the cultivation of large volumes of the pathogen during the production process instead. Moreover, the development period of traditionally manufactured vaccines is rather long because for each pathogenic candidate a new production method needs to be designed and tested. A very promising concept that can tackle the problems that arise with these traditional methodologies is a vaccine platform. In a vaccine platform, the pathogen is not grown, but a selected protective part of the pathogen (antigen) is produced instead. Importantly, the platform that forms the basis of the vaccine can be maintained and used for the development of any new vaccine (Figure 1). In other words: the production process of new vaccines is essentially the same as the production process of other vaccines based on the vaccine platform. As such, vaccine platforms have the potential to drive the development of safe and effective vaccines in less time and at a lower cost. The initial development of robust and high-yielding production processes is of major importance for vaccine platforms to form a solution to the increasing demand for vaccines.

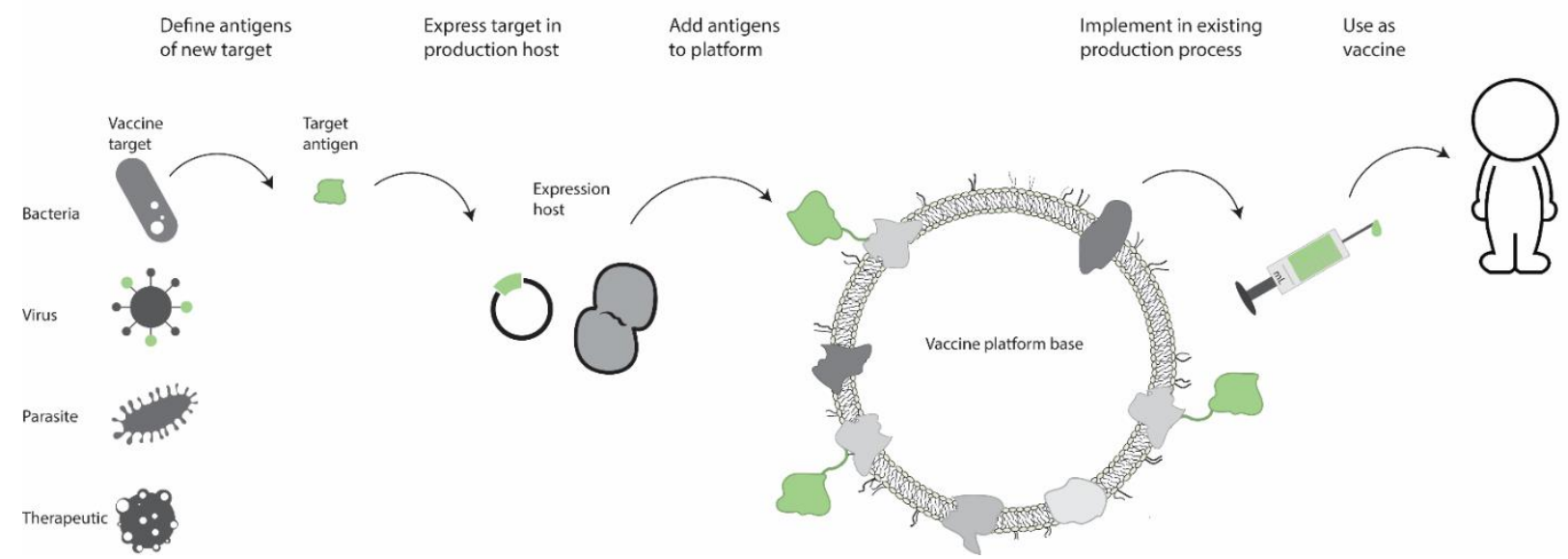

Figure 1. Basic concept of a vaccine platform. Antigens against varying targets can be exchanged on the vaccine platform, in this case an outer membrane vesicle (OMV). This new vaccine can be produced in the well-defined production process of the carrier OMV, since the addition of an antigen should minimally affect this process.

\section{Outer membrane vesicle-based vaccine platforms}

Bacterial outer membrane vesicles (OMVs) are highly suitable as a basis for the development of a vaccine platform. OMVs are 20-200 nm sized vesicles shed by Gramnegative bacteria and consist of a lipid bilayer, outer membrane proteins and OMV cargo [13]. The membrane of the vesicle consists of phospholipids, lipopolysaccharides 
(LPS), and proteins (Figure 2). The lumen of the vesicle has been shown to contain cytoplasmic components as well as ribonucleic acid (RNA), and deoxyribonucleic acid (DNA). Biologically, OMVs are generally thought to be advantageous to the survival chances of the bacterium. OMVs can deliver virulence factors, modulate host immune responses, mediate cell-to-cell communication, or be involved in nutrient acquisition [13-15]. Moreover, OMVs can provide protection for the bacterium against the infection of bacteriophages, which are viruses that infect bacteria. This has been shown for $V$. cholerae where OMVs inhibit bacteriophage infection by forming OMVs as a decoy [16]. OMVs offer a combination of the most important characteristics of vaccines, making these nanoparticles intriguing vaccine-platform candidates. The surface of OMVs is

Outer membrane vesicle

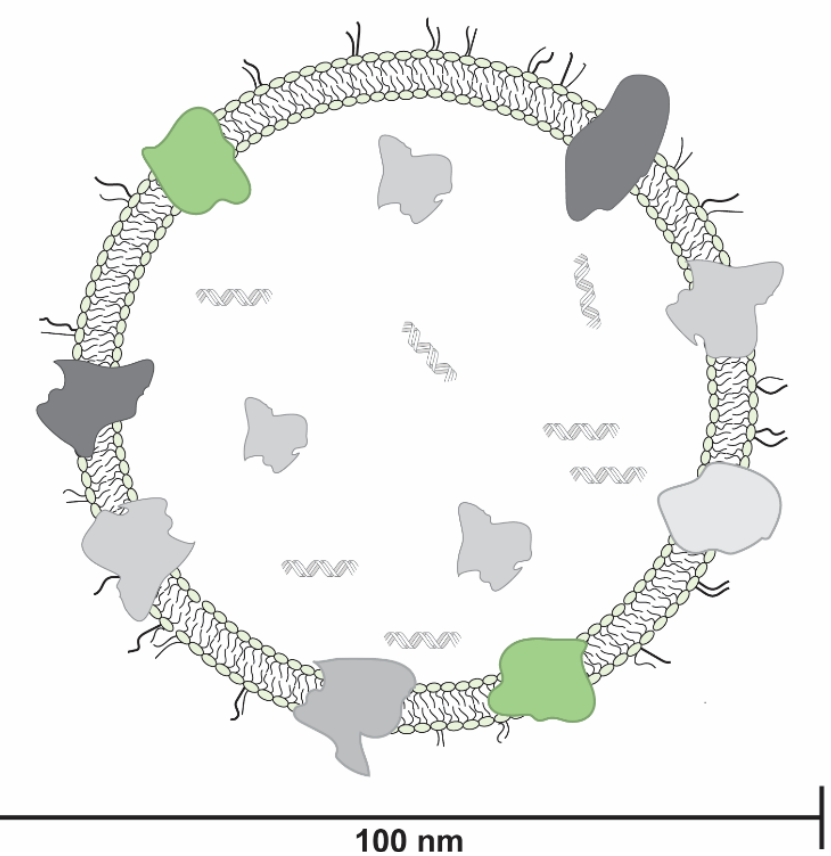

lipopolysaccharide phospholipid outer membrane protein cytosolic protein DNA / RNA

Figure 2. Outer membrane vesicle composition. OMVs are spherical nanoparticles secreted from bacteria. An OMV consists of a membrane with lipopolysaccharides, phospholipids, and outer membrane proteins. The lumen of the vesicle is filled with components as proteins, DNA, and RNA.

filled with immunogenic components of the bacterium from which the vesicles have been derived [17-19], but because OMVs are non-replicative particles, they cannot cause the disease. As such, OMV-based vaccines can be regarded as safe. A second advantage is the particle shape of OMVs in combination with their ability to activate the innate immune system, which makes OMVs self-adjuvating [14]. The OMVs are filled with components that act as pathogen-associated molecular patters (PAMPS), such as LPS and DNA [20]. These components can be recognized by the human immune system 
and induce infection-like innate immune response. The small size and particle shape of OMVs allows for distribution throughout the body, resulting in a systemic innate immune response [21]. Another advantage of OMV-based vaccines is that OMVs are highly stable at varying temperatures [22-24], which facilitates storage and transport of OMV-based vaccines. OMVs have been shown to elicit long-term memory responses [25], which are important for the efficacy of the vaccine. Lastly, OMVs induce both humoral and cellular mediated immune responses against OMV-presented antigens. These immunogenic properties of OMVs distinguish OMV-based vaccines from other vaccine types that have been regarded as highly safe, such as subunit vaccines. Subunit vaccines are a combination of a purified antigen formulated with an adjuvating agent and are regarded as a highly safe due to their high purity. However, the induced immune response of subunit vaccines is often low. Various research is performed to optimize subunit vaccines, by finding a perfect mix of adjuvant agents and formulation for each new vaccine [26]. Expressing antigens on OMVs combines the advantages of the high purity of subunit vaccines with balanced immune responses.

\section{Design of a Neisseria meningitidis OMV-based vaccine platform}

OMVs have been used to control epidemic outbreaks of Neisseria meningitidis (Nm) serogroup B meningitis in Norway, Chile, Cuba, Brazil, and New-Zealand [27-32]. Despite the experience with OMV-based vaccines during outbreaks and over 30 years of research on OMV-based vaccines, only recently the first vaccine containing an OMV, the meningococcal serogroup B vaccine $4 \mathrm{CMenB}$, has been approved by the FDA and EMA for routine use [33].

New vaccines based on a modular OMV-based vaccine platform should express specific disease-associated antigens. A vaccine platform based on the OMVs from the pathogen N. meningitidis rules out possible side-effects against commensal bacteria. In contrast, heterologous antigens can be expressed on the laboratory workhorse E. coli derived OMVs $[34,35]$. Expression of antigens in E. coli is relatively straightforward, although vaccines based on these OMVs could cause unwanted immune responses against commensal strains of E. coli. OMVs derived from Nm have been shown safe and genetic modification of Neisseria spp. is feasible [36].

In this thesis we study Nm serogroup B OMVs as a basis for a vaccine platform. To develop such a vaccine platform, heterologous antigens were produced by expression of 
the heterologous antigen in the OMV producing Nm strain. Surface localization was reached through fusion of the antigen to an $\mathrm{N}$-terminal part of the $\mathrm{Nm}$ factor $\mathrm{H}$ binding protein (fHbp)[37]. In this way, OMVs can be produced with surface exposed heterologous antigens. In addition to the expression of heterologous antigens, multiple modifications are required to develop a vaccine platform base. In this thesis, a $\mathrm{Nm}$ production strain was used based on a Nm serogroup B isolate, which has been the basis of the serogroup B meningococcal vaccines HexaMen $₫$ and NonaMen $₫$. These meningococcal vaccines have been developed at Intravacc (and its predecessors) [38], where the work of this thesis has been performed. This pathogen has been modified in such a way that its OMVs can be used as the basis for a modular vaccine platform. These modifications are made to improve OMV formation, reduce the endotoxicity, modulate the immune response, and express heterologous antigens on the surface of the OMVs. The modifications to the Nm OMV producing strain and the rationale behind them are described below.

1. Increased OMV formation

The OMV productivity was enhanced artificially by stimulating the release of OMVs from the bacterium by reducing the linkage between the outer membrane and the peptidoglycan layer. For Nm, a knock-out mutation of the rmpM gene causes reduced linkage of the outer membrane to the peptidoglycan layer and stimulates OMV release (Figure 3). The knockout of $r m p M$ has resulted in a 2-fold increase in the production of detergent extracted OMV and a 10-fold increase of the production of OMV from the culture supernatant [39]. 

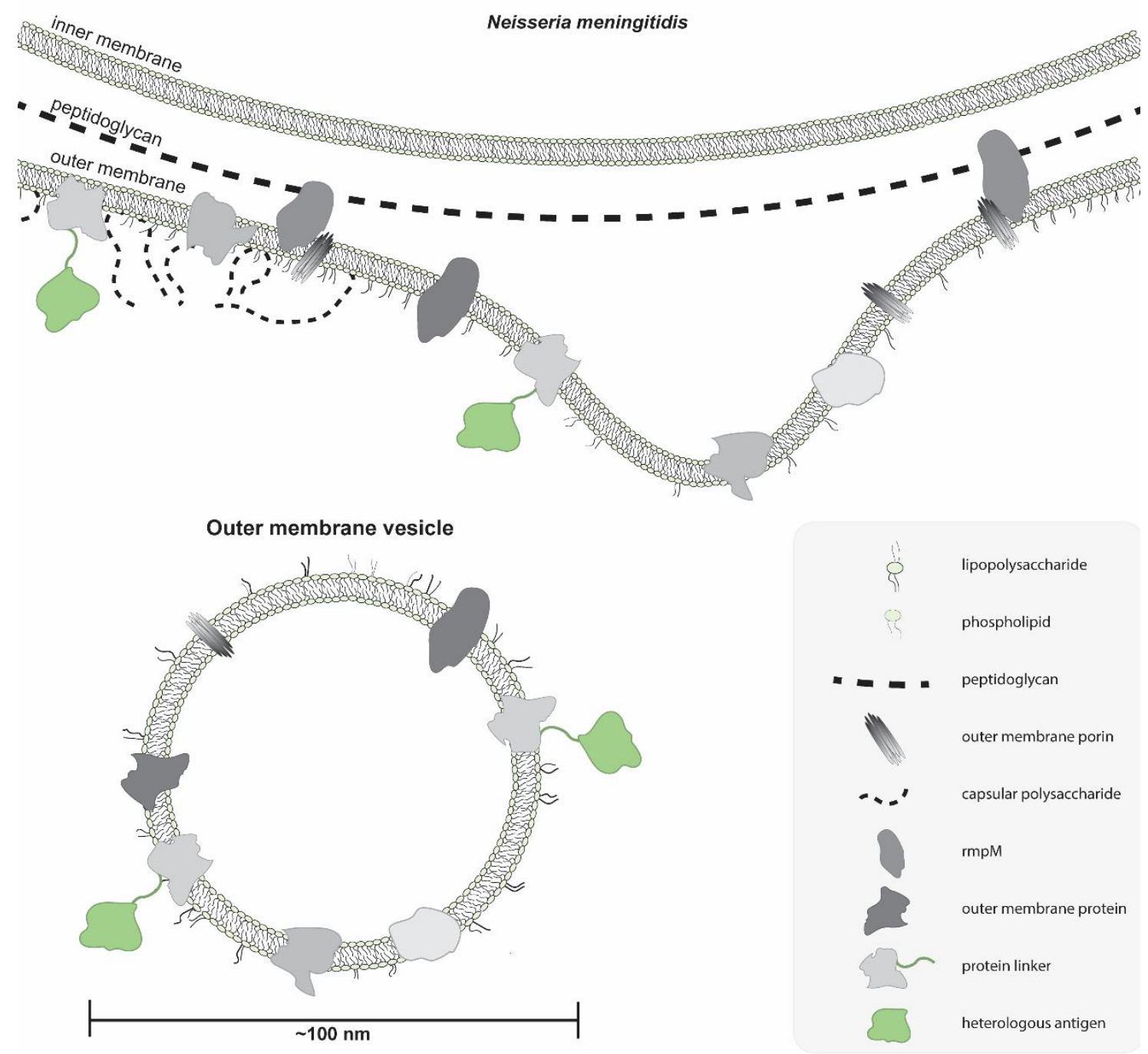

Figure 3. Design of a Neisseria meningitidis OMV-based vaccine platform. Outer membrane vesicles (OMVs) are nanoparticles formed by blebbing of the outer membrane. Heterologous protein antigens are expressed and directed to the outer membrane. Blebbing is induced by reduced linkage of the outer membrane to the peptidoglycan layer to stimulate OMV release (1). LPS is modulated to lower its toxicity and modulate the activation of the innate immune system (2). LPS is further modified to stimulate the induced antigen specific immune response by enhancing the binding to DC-SIGN (3). The capsular polysaccharide is removed for enhanced safety during production (4), Lastly, the Neisserial antigen PorA is removed to direct immune responses against heterologous antigens (5).

\section{Reduced endotoxicity}

A proposed biological role of OMVs is the delivery of bacterial virulence factors to the host [40]. Lipopolysaccharides are an important virulence factor of $\mathrm{Nm}$ that can trigger intense inflammation and result in septic shock [41]. LPS activates the innate immune system by activation of the Toll-like receptor 4 (TLR4) [42], which results in 
proinflammatory cell activation. To be able to use OMVs as vaccine, the LPS needs to be severely reduced or detoxified. Reduction of LPS has been reached by the production of OMVs by detergent extraction of bacteria. Immune modulation by the genetic modification of LPS allows the engineering of the degree of TLR4 activation [43] and is required for the direct use of spontaneous released OMVs. Genetic modification of the IpxL1 gene results in penta-acylated lipid A LPS instead of hexa-acylated LPS [44]. This penta-acylated LPS shows reduced endotoxicity, making detergent extraction to reduce excess LPS reactogenicity obsolete [39].

3. Skewing of T-cell responses

Besides being a potent adjuvant, Nm LPS can selectively induce the desired arm of the immune response. Targeting vaccines directly to dendritic cells, that efficiently take up antigens and stimulate antigen specific immune responses, is a method to enhance the potency of vaccines [45]. LPS-deficient mutants are poorly internalized in dendritic cells and lack the ability to activate dendritic cells [46]. LPS can be further modified to exhibit strong adhesion and internalization properties towards dendritic cells [47]. An lgtB gene knockout results in a truncated oligosaccharide LPS form, which improves the interacting with DC-SIGN, and thus enhancing phagocytosis [48]. OMVs containing LgtB mutated LPS in combination with penta-acylated lipid A showed increased uptake by dendritic cells and maturation of dendritic cells. Alternatively, LPS from N. gonorrhoeae expressing a terminal $\mathrm{N}$-acetylgalactosamine results in skewing towards $\mathrm{T}$-helper type $2\left(\mathrm{~T}_{\mathrm{H}} 2\right)$ responses [49]. Here we use a LgtB LPS mutant of Nm to enhance the antigen specific immune response.

4. Prevention of auto-immune responses

The capsular polysaccharide of $\mathrm{Nm}$ is used as the basis of $\mathrm{Nm}$ serogroup A, C, W, and Y vaccines [50]. The capsular polysaccharide (CPS) of Nm serogroup B consists of $\alpha(2-8)$ linked $\mathrm{N}$-acetylneuraminic acid polymers, that are also found on human neural cells [51]. Although the serogroup B capsule is not immunogenic due to immune tolerance, including serogroup B capsular polysaccharide in a vaccine composition could lead to autoimmunity [52]. Bacterial capsules prevent phagocytosis, thus removing the $\mathrm{Nm}$ capsule results in reduced virulence of the bacteria [53]. Removing the Nm capsule rules out possible autoimmunity and it enhances the safety of the production host, which is a practical advantage in development and production in the laboratory. 
Capsule deficient mutants of Nm can be obtained by deletion of the complete cps locus $[54,55]$, or by knockout mutation of the siaD gene [56]. SiaD is involved in the synthesis of $\alpha(2,8)$ polysialic acids [57]. A SiaD knockout mutant was used as the basis of the Nm OMV platform.

\section{Reduced OMV-backbone antigenicity}

A vaccine platform should have reduced immune responses against the carrier platform to optimize the response against heterologous antigens and to be able to reuse the platform backbone for subsequent vaccines. Nm outer membrane porin A (PorA) is the major protein component of Nm OMVs and identified as a major antigenic protein [58]. Serum bactericidal antibodies directed against PorA correlate with protection against meningococcal disease [59]. Nm serogroup B meningitis vaccines expressing multiple PorA variants have been developed [60-62]. In this thesis a Nm strain without PorA was used to improve the immune response against heterologous antigens.

\section{OMV production processes}

OMVs as the basis of a modular vaccine platform can provide important standardization of the elicited immune responses, as well as the delivery of antigens on nanoparticles. A vaccine platform based on OMVs spontaneously released from the bacteria (sOMVs) is only viable if the production yields are high. Currently, OMVs are regarded as expensive vaccine component to produce $[63,64]$. These OMV production processes have moderate yields as these first-generation OMVs were based on extraction with the detergent deoxycholate to reduce the endotoxic lipopolysaccharide content of OMVs [65]. In the production process, crude yields are high although the detergent extraction reduces the content of important protective lipoprotein antigens and impairs vesicle integrity [63]. This results in aggregation upon cooled storage and subsequent difficulties in sterile filtration of the product. Additionally, deoxycholate used for OMV extraction is a product of animal origin that remains in the vesicle product and is thus undesirable [66]. OMVs spontaneously released by bacteria retain lipoproteins in their vesicles. Purifying OMVs from the supernatant of bacterial cultures would be preferred because they better mimic the natural situation, have improved stability, and have a shortened purification process. Despite these advantages, current Nm sOMV production processes are low yielding. As the release of OMVs by the bacterium mimics their 
biological role, research in the biogenesis of OMVs can result in biologically inspired and improved production processes.

Recently, the proposed mechanisms of OMV biogenesis have been categorized in to three models [67]. One model is based on OMV release through altered lipid asymmetry in the outer membrane, which normally consist of LPS in the outer leaflet and phospholipids in the inner leaflet. Phospholipid accumulation in the outer leaflet of the outer membrane results in OMV release. A second model describes that OMVs are released by accumulation of misfolded and unfolded proteins in the periplasm. The third model describes OMV formation because of LPS modifications that impair the stability of the outer membrane. It remains unclear if the biogenesis of OMVs is shared among bacterial species. For Nm it was previously shown that cysteine depletion results in the release of OMVs in a batch culture [68]. Cysteine depletion caused impaired sulfur supply, the onset of the stationary growth phase, and transcriptome responses of oxidative stress. This state was accompanied with increased OMV release although the exact mechanism of OMV release remained unknown. To improve the yield of Nm OMV production, more understanding of the biogenesis of OMVs is required.

\section{Aim and thesis outline}

The aim of this thesis is to obtain a better understanding of outer membrane vesicle formation by Neisseria meningitidis and OMV quality, and use this to develop improved OMV production processes that can become a cost-effective basis for an OMV-based vaccine platform.

In Chapter 2 we review the possibilities of an OMV-based vaccine platform. We assess the possibilities of introducing heterologous antigens to the OMV and assess the effect of the antigen location on the immune system and production process. To enhance the development of OMV production systems, analytical tools for direct OMV quantification method are required. In Chapter 3, a nanoparticle characterization method was assessed and made suitable for the analysis of OMVs. To improve the volumetric productivity both the biomass concentration and the specific OMV productivity can be increased. This thesis focusses on improving the OMV release of the individual bacterium. Based on the research on cysteine depletion as trigger of OMV release, we hypothesized that sulfur depletion in general causes OMV release. Chapter 4 researches the role of the sulfur source and the effect of sulfur depletion on the release of OMVs. 
Sulfur sources with different oxidation states were used as the intracellular redox state was hypothesized to be involved in the release of OMV. Additionally, oxidative stress responses have been observed previously upon OMV release after cysteine depletion. In Chapter 5, the effect of oxidative stress on the release of OMVs was studied by applying increased dissolved oxygen concentrations directly to the cultures. In Chapter 6, a new production process of OMVs was researched, based on the OMV stimulating triggers found in the previous chapters. The possibility of producing heterologous antigens in this process was tested. The OMVs were characterized to assess whether the vesicles had sufficient quality to be used as vaccines. Further process intensification of OMV production was studied in Chapter 7. The volumetric yield of OMV production in bioreactors was improved by applying triggers of OMV release in a continuous cultivation process. This continuous production approach of OMVs allows the development of vaccines for low-and middle-income countries as well as other low-cost applications of OMVs. Lastly, Chapter 8 discusses the major findings in this thesis and provides an outlook of the future of OMV-based vaccine platforms.

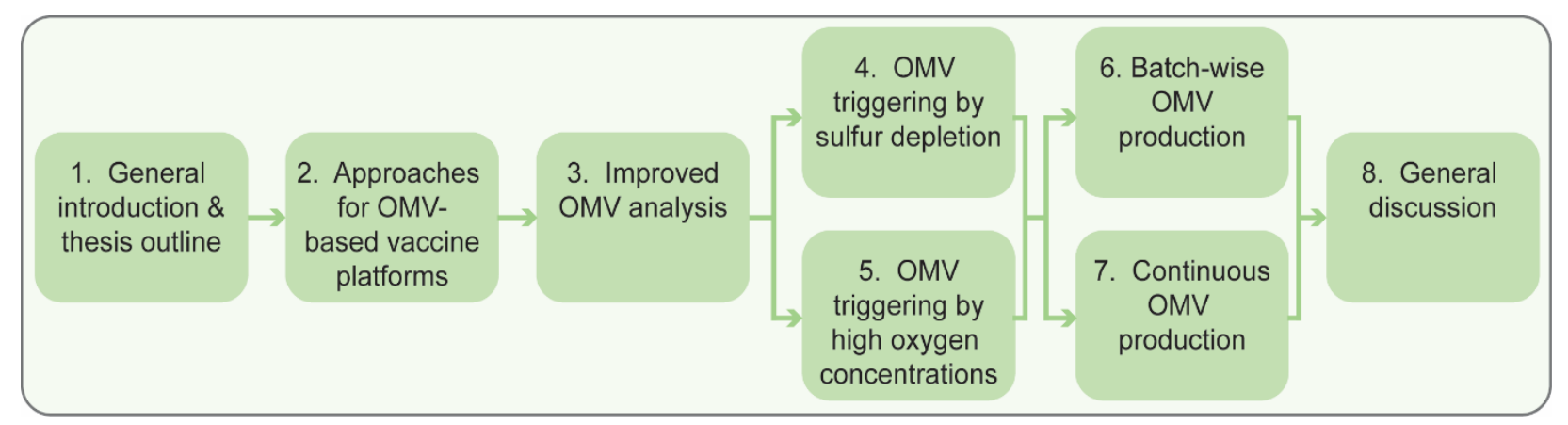

Figure 4: Graphical outline of the chapters in this thesis. An overview of the current state of research on OMV-based vaccine platform is given in Chapter 2. Chapter 3 describes an improved method of OMV quantification that is required for the research in the following chapters. Chapter 4 and 5 research the release of OMVs from bacteria. The triggers found in these chapters are applied in two different approaches of OMV production. Batch-wise OMV production is used to provide a proof of concept for producing heterologous antigens on OMVs in Chapter 6. In Chapter 7, continuous OMV production is researched to further improve the productivity, followed by a general discussion in Chapter 8. 


\section{References}

1. Plotkin's Vaccines. Vol. 7th Edition. 2018.

2. Mariner, J.C., et al., Rinderpest Eradication: Appropriate Technology and Social Innovations. Science, 2012. 337(6100): p. 1309-1312.

3. Henderson, D.A., The eradication of smallpox - An overview of the past, present, and future. Vaccine, 2011. 29: p. D7-D9.

4. Kew, O. and M. Pallansch, Breaking the Last Chains of Poliovirus Transmission: Progress and Challenges in Global Polio Eradication. Annual Review of Virology, 2018. 5(1): p. 427-451.

5. Gambhir, M., et al., A Change in Vaccine Efficacy and Duration of Protection Explains Recent Rises in Pertussis Incidence in the United States. PLOS Computational Biology, 2015. 11(4): p. e1004138.

6. Cohen, M.L., Epidemiology of Drug Resistance: Implications for a Post-Antimicrobial Era. Science, 1992. 257(5073): p. 1050-1055.

7. Lowy, F.D., Antimicrobial resistance: the example of Staphylococcus aureus. The Journal of Clinical Investigation, 2003. 111(9): p. 1265-1273.

8. Rice, L.B., Antimicrobial resistance in gram-positive bacteria. American Journal of Infection Control, 2006. 34(5, Supplement): p. S11-S19.

9. Morens, D.M., G.K. Folkers, and A.S. Fauci, The challenge of emerging and re-emerging infectious diseases. Nature, 2004. 430: p. 242.

10. Schiffman, M., et al., Human papillomavirus and cervical cancer. The Lancet, 2007. 370(9590): p. 890907.

11. Laitinen, O.H., et al., Coxsackievirus B1 Is Associated With Induction of $\beta$-Cell Autoimmunity That Portends Type 1 Diabetes. Diabetes, 2013.

12. Sahin, U. and Ö. Türeci, Personalized vaccines for cancer immunotherapy. Science, 2018. 359(6382): p. 1355.

13. Kulp, A. and M.J. Kuehn, Biological Functions and Biogenesis of Secreted Bacterial Outer Membrane Vesicles. Annual Review of Microbiology, 2010. 64(1): p. 163-184.

14. Kaparakis-Liaskos, M. and R.L. Ferrero, Immune modulation by bacterial outer membrane vesicles. Nature Reviews Immunology, 2015. 15: p. 375.

15. Berleman, J. and M. Auer, The role of bacterial outer membrane vesicles for intra- and interspecies delivery. Environmental Microbiology, 2012. 15(2): p. 347-354.

16. Reyes-Robles, T., et al., Vibrio cholerae outer membrane vesicles inhibit bacteriophage infection. Journal of Bacteriology, 2018.

17. Alaniz, R.C., et al., Membrane Vesicles Are Immunogenic Facsimiles of Salmonella typhimurium That Potently Activate Dendritic Cells, Prime B and T Cell Responses, and Stimulate Protective Immunity In Vivo. The Journal of Immunology, 2007. 179(11): p. 7692.

18. Veith, P.D., et al., Porphyromonas gingivalis Outer Membrane Vesicles Exclusively Contain Outer Membrane and Periplasmic Proteins and Carry a Cargo Enriched with Virulence Factors. Journal of Proteome Research, 2014. 13(5): p. 2420-2432.

19. Tani, C., et al., Quantification by LC-MSE of outer membrane vesicle proteins of the Bexsero® vaccine. Vaccine, 2014. 32(11): p. 1273-1279.

20. Kuehn, M.J. and N.C. Kesty, Bacterial outer membrane vesicles and the host-pathogen interaction. Genes \& Development, 2005. 19(22): p. 2645-2655.

21. Bitto, N.J. and M. Kaparakis-Liaskos, The Therapeutic Benefit of Bacterial Membrane Vesicles. International journal of molecular sciences, 2017. 18(6): p. 1287.

22. Arigita, C., et al., Stability of mono- and trivalent meningococcal outer membrane vesicle vaccines. Vaccine, 2004. 22(5): p. 629-642.

23. Kanojia, G., et al., Development of a thermostable spray dried outer membrane vesicle pertussis vaccine for pulmonary immunization. Journal of Controlled Release, 2018. 286: p. 167-178.

24. Alves, N.J., et al., Protecting enzymatic function through directed packaging into bacterial outer membrane vesicles. Scientific Reports, 2016. 6: p. 24866.

25. Romeu, B., et al., Combined meningococcal serogroup A and W135 outer-membrane vesicles activate cellmediated immunity and long-term memory responses against non-covalent capsular polysaccharide A. Immunologic Research, 2014. 58(1): p. 75-85.

26. Perrie, Y., et al., Vaccine adjuvant systems: Enhancing the efficacy of sub-unit protein antigens. International Journal of Pharmaceutics, 2008. 364(2): p. 272-280.

27. Bjune, G., et al., Effect of outer membrane vesicle vaccine against group B meningococcal disease in Norway. The Lancet, 1991. 338(8775): p. 1093-1096. 
28. Sierra, G.V., et al., Vaccine against group B Neisseria meningitidis: protection trial and mass vaccination results in Cuba. NIPH annals, 1991. 14(2): p. 195-207; discussion 208-10.

29. Cassio de Moraes, J., et al., Protective efficacy of a serogroup B meningococcal vaccine in Sao Paulo, Brazil. The Lancet, 1992. 340(8827): p. 1074-1078.

30. Thornton, V., et al., Safety and immunogenicity of New Zealand strain meningococcal serogroup B OMV vaccine in healthy adults: Beginning of epidemic control. Vaccine, 2006. 24(9): p. 1395-1400.

31. Boslego, J., et al., Efficacy, safety, and immunogenicity of a meningococcal group B (15:P1.3) outer membrane protein vaccine in Iquique, Chile. Vaccine, 1995. 13(9): p. 821-829.

32. Holst, J., et al., Properties and clinical performance of vaccines containing outer membrane vesicles from Neisseria meningitidis. Vaccine, 2009. 27: p. B3-B12.

33. Serruto, D., et al., The new multicomponent vaccine against meningococcal serogroup $B, 4 C M e n B$ : Immunological, functional and structural characterization of the antigens. Vaccine, 2012. 30: p. B87-B97.

34. Kesty, N.C. and M.J. Kuehn, Incorporation of Heterologous Outer Membrane and Periplasmic Proteins into Escherichia coli Outer Membrane Vesicles. Journal of Biological Chemistry, 2004. 279(3): p. 2069-2076.

35. Kim, J.-Y., et al., Engineered bacterial outer membrane vesicles with enhanced functionality. Journal of molecular biology, 2008. 380(1): p. 51-66.

36. Dillard, J.P., Genetic Manipulation of Neisseria gonorrhoeae. Current protocols in microbiology, 2011.0 4: p. Unit4A.2-Unit4A.2.

37. Salverda, M.L.M., et al., Surface display of a borrelial lipoprotein on meningococcal outer membrane vesicles. Vaccine, 2016. 34(8): p. 1025-1033.

38. Intravacc. Factsheet Outer membrane vesicles (OMVs). 2016 July 2016 [cited 2018 25/10]; Available from: www.intravacc.nl/media/1233/20160712-factsheet-omvs.pdf.

39. van de Waterbeemd, B., et al., Improved OMV vaccine against Neisseria meningitidis using genetically engineered strains and a detergent-free purification process. Vaccine, 2010. 28(30): p. 4810-6.

40. Bomberger, J.M., et al., Long-Distance Delivery of Bacterial Virulence Factors by Pseudomonas aeruginosa Outer Membrane Vesicles. PLOS Pathogens, 2009. 5(4): p. e1000382.

41. Raetz, C.R.H. and C. Whitfield, Lipopolysaccharide Endotoxins. Annual Review of Biochemistry, 2002. 71(1): p. 635-700.

42. Takeuchi, O., et al., Differential Roles of TLR2 and TLR4 in Recognition of Gram-Negative and GramPositive Bacterial Cell Wall Components. Immunity, 1999. 11(4): p. 443-451.

43. Zariri, A., et al., Modulating endotoxin activity by combinatorial bioengineering of meningococcal lipopolysaccharide. Scientific Reports, 2016. 6: p. 36575.

44. van der Ley, P., et al., Modification of lipid A biosynthesis in Neisseria meningitidis lpxL mutants: influence on lipopolysaccharide structure, toxicity, and adjuvant activity. Infect Immun, 2001. 69(10): p. 5981-90.

45. Foged, C., A. Sundblad, and L. Hovgaard, Targeting Vaccines to Dendritic Cells. Pharmaceutical Research, 2002. 19(3): p. 229-238.

46. Uronen-Hansson, H., et al., Human dendritic cell activation by Neisseria meningitidis: phagocytosis depends on expression of lipooligosaccharide (LOS) by the bacteria and is required for optimal cytokine production. Cellular Microbiology, 2004. 6(7): p. 625-637.

47. Kurzai, O., et al., Carbohydrate composition of meningococcal lipopolysaccharide modulates the interaction of Neisseria meningitidis with human dendritic cells. Cellular Microbiology, 2005. 7(9): p. 1319-1334.

48. Steeghs, L., et al., Neisseria meningitidis expressing lgtB lipopolysaccharide targets DC-SIGN and modulates dendritic cell function. Cellular Microbiology, 2005. 8(2): p. 316-325.

49. van Vliet, S.J., et al., Variation of Neisseria gonorrhoeae Lipooligosaccharide Directs Dendritic CellInduced T Helper Responses. PLOS Pathogens, 2009. 5(10): p. e1000625.

50. Jennings, H.J. Capsular Polysaccharides as Vaccine Candidates. in Bacterial Capsules. 1990. Berlin, Heidelberg: Springer Berlin Heidelberg.

51. Finne, J., et al., An IgG monoclonal antibody to group B meningococci cross-reacts with developmentally regulated polysialic acid units of glycoproteins in neural and extraneural tissues. The Journal of Immunology, 1987. 138(12): p. 4402-4407.

52. Nedelec, J., et al., Evidence for autoimmune antibodies directed against embryonic neural cell adhesion molecules (N-CAM) in patients with group B meningitis. Journal of Neuroimmunology, 1990. 29(1): p. 4956.

53. Spinosa, M.R., et al., The Neisseria meningitidis Capsule Is Important for Intracellular Survival in Human Cells. Infection and Immunity, 2007. 75(7): p. 3594-3603.

54. Van Der Ley, P. and J.T. Poolman, Construction of a multivalent meningococcal vaccine strain based on the class 1 outer membrane protein. Infection and Immunity, 1992. 60(8): p. 3156-3161. 
55. Bos, M.P. and J. Tommassen, Viability of a Capsule- and Lipopolysaccharide-Deficient Mutant of Neisseria meningitidis. Infection and Immunity, 2005. 73(9): p. 6194-6197.

56. Steeghs, L., et al., Outer membrane composition of a lipopolysaccharide-deficient Neisseria meningitidis mutant. The EMBO Journal, 2001. 20(24): p. 6937-6945.

57. Edwards, U., et al., Molecular analysis of the biosynthesis pathway of the $\alpha-2,8$ polysialic acid capsule by Neisseria meningitidls serogroup B. Molecular Microbiology, 1994. 14(1): p. 141-149.

58. van de Waterbeemd, B., et al., Quantitative Proteomics Reveals Distinct Differences in the Protein Content of Outer Membrane Vesicle Vaccines. Journal of Proteome Research, 2013. 12(4): p. 1898-1908.

59. Rouppe van der Voort, E., et al., Immunogenicity studies with a genetically engineered hexavalent PorA and a wild-type meningococcal group B outer membrane vesicle vaccine in infant cynomolgus monkeys. Vaccine, 2000. 18(14): p. 1334-1343.

60. van der Ley, P., J. van der Biezen, and J.T. Poolman, Construction of Neisseria meningitidis strains carrying multiple chromosomal copies of the porA gene for use in the production of a multivalent outer membrane vesicle vaccine. Vaccine, 1995. 13(4): p. 401-407.

61. Kaaijk, P., et al., Preclinical safety and immunogenicity evaluation of a nonavalent PorA native outer membrane vesicle vaccine against serogroup B meningococcal disease. Vaccine, 2013. 31(7): p. 10651071.

62. van de Waterbeemd, B., et al., Improved Production Process for Native Outer Membrane Vesicle Vaccine against Neisseria meningitidis. PLOS ONE, 2013. 8(5): p. e65157.

63. van der Pol, L., M. Stork, and P. van der Ley, Outer membrane vesicles as platform vaccine technology. Biotechnology Journal, 2015. 10(11): p. 1689-1706.

64. Tan, K., et al., Outer Membrane Vesicles: Current Status and Future Direction of These Novel Vaccine Adjuvants. Frontiers in Microbiology, 2018. 9: p. 783.

65. Frasch, C.E., Vaccines for prevention of meningococcal disease. Clinical Microbiology Reviews, 1989. 2(Suppl): p. S134-S138.

66. Claassen, I., et al., Production, characterization and control of a Neisseria meningitidis hexavalent class 1 outer membrane protein containing vesicle vaccine. Vaccine, 1996. 14(10): p. 1001-1008.

67. Volgers, C., P.H.M. Savelkoul, and F.R.M. Stassen, Gram-negative bacterial membrane vesicle release in response to the host-environment: different threats, same trick? Critical Reviews in Microbiology, 2018. 44(3): p. 258-273.

68. van de Waterbeemd, B., et al., Cysteine depletion causes oxidative stress and triggers outer membrane vesicle release by Neisseria meningitidis; implications for vaccine development. PLoS One, 2013. 8(1): p. e54314. 


\section{Chapter 2}

\section{Bioengineering bacterial outer membrane vesicles as vaccine platform}

Published as:

Gerritzen, M.J.H., D.E. Martens, R.H. Wijffels, L. van der Pol and M. Stork (2017).

"Bioengineering bacterial outer membrane vesicles as vaccine platform."

Biotechnology Advances Volume 35, Issue 5, September 2017, Pages 565-574 


\begin{abstract}
Outer membrane vesicles (OMVs) are naturally non-replicating, highly immunogenic spherical nanoparticles derived from Gram-negative bacteria. OMVs from pathogenic bacteria have been successfully used as vaccines against bacterial meningitis and sepsis among others and the composition of the vesicles can easily be engineered. OMVs can be used as a vaccine platform by engineering heterologous antigens to the vesicles. The major advantages of adding heterologous proteins to the OMV are that the antigens retain their native conformation, the ability of targeting specific immune responses, and a single production process suffices for many vaccines. Several promising vaccine platform concepts have been engineered based on decorating OMVs with heterologous antigens. This review discusses these vaccine concepts and reviews design considerations as the antigen location, the adjuvant function, physiochemical properties, and the immune response
\end{abstract}

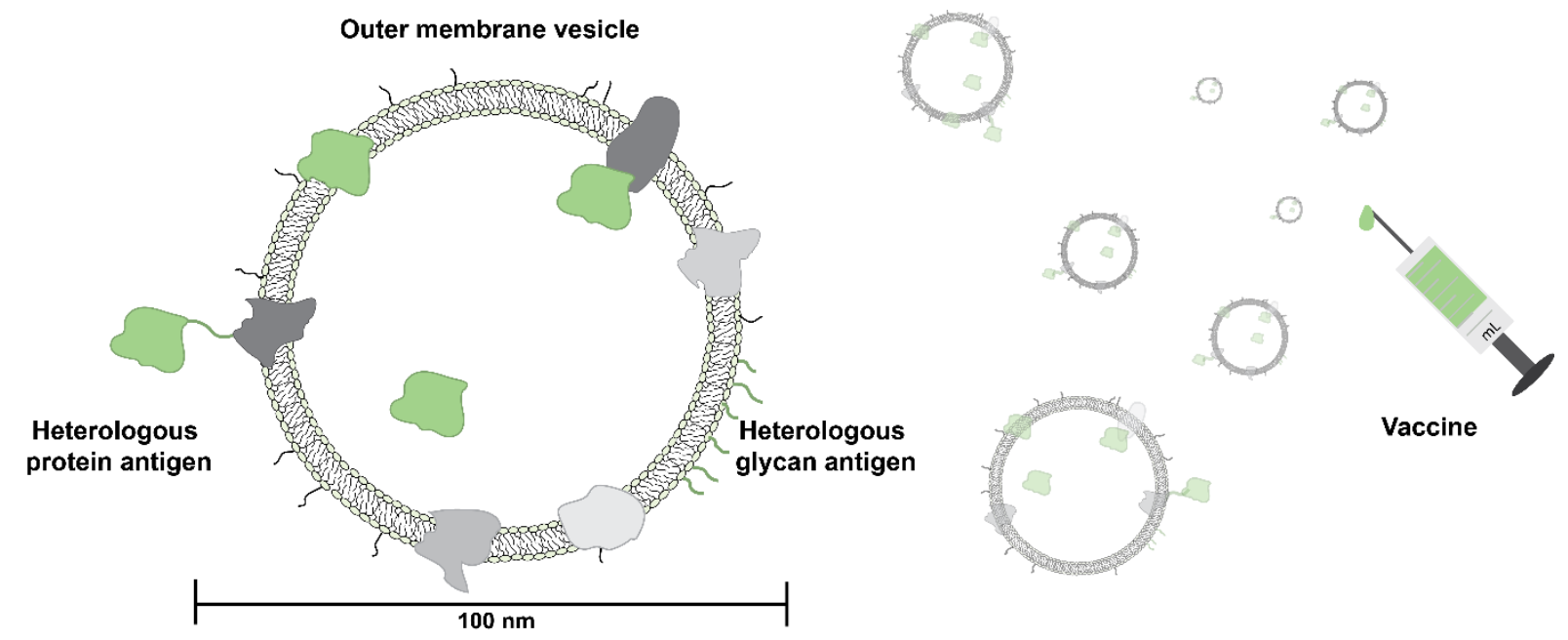




\section{Vaccine platforms}

Many vaccines are developed based on Pasteur's principle: "isolate, inactivate and inject" or by the selection of attenuated strains [6]. Although many very effective vaccines have been developed by these methods, they have often led to adverse effects. Nowadays, vaccines must combine the lowest possible adverse effects with a high efficacy. Vaccine platforms can standardize the provoked immune response with high safety and low adverse effects, while the design allows for easy switching of displayed antigens leading to high efficacy. Protection against different diseases can be addressed by presenting different antigens.

The development of a highly safe and effective vaccine requires a lot of resources and time. At the same time the upcoming post-antibiotic era may require the development of more vaccines that also need to be developed in a short period [7]. The development trajectory can be shortened by vaccine platforms [9], since these platforms provide a blueprint for development of many different vaccines instead of a specific development trajectory for each separate vaccine with all its uncertainties. The safety of a vaccine platform can be established by thorough development of the platform itself. Once the safety and efficacy of a platform has been established, development time can be reduced for new vaccines, since less testing will be required and unexpected failures will occur less. This advantage of vaccine platforms will reduce significantly the time to market, which is notoriously long for new vaccines.

A vaccine platform should provoke a strong specific immune response. This response is triggered by conformationally correct antigen presentation, PAMPs (see glossary) to activate antigen presenting cells, and a nanosized particulate nature. Furthermore, it should be possible to easily add antigens onto the vaccine platform. Platform nanovaccines can be based on many components, for example VLPs, ISCOMs, polymeric nanoparticles, inorganic nanoparticles, liposomes, and emulsions. A component that is often overlooked are OMVs [10]. While many nanoparticles are capable of transferring heterologous antigens to antigen presenting cells, the ability to properly stimulate the immune system is often not natively present [11]. OMVs, however, combine antigen presentation with proper adjuvant properties, making them highly suitable as a vaccine platform. 
OMVs are non-replicative vesicles that are naturally produced by Gram-negative bacteria and contain excellent intrinsic immunostimulatory properties based on their particulate nature and composition [12]. The vesicles consist of phospholipids, lipopolysaccharides (LPS), outer membrane proteins and entrapped periplasmic components [13]. OMVs are ascribed many biological functions such as cell to cell communication, surface modifications and the expulsion of components [13]. Overall, OMVs have been shown to be highly stable even upon elevated temperatures and several chemical treatments [14]. This review addresses the latest state of research with respect to the development of an OMV based vaccine platform. First, we discuss the location of the antigen, which is either inside the OMV or displayed on the OMV surface. Location is important for the provoked type of immune response. Two

approaches of location specific antigen addition are discussed, namely the endogenous addition based on antigen production by the bacterium itself and the exogenous methods that introduce the antigen in a separate process step. The bioengineering of the provoked immune response and the endotoxicity is discussed. Additionally, we discuss the bioengineering of the physiochemical properties of the OMV and the potential of outer membrane vesicles as vaccine platforms. Lastly, we propose a uniform naming of different vesicles based on the origin of the OMVs. 


\section{Designing the OMV: antigen location}

Heterologous antigens on OMVs can be presented with or without surface exposure, attached to the vesicle or non-attached and directly produced by the bacterium or combined in a later production stage. Various possibilities of antigen locations and their production method are schematically shown in Figure 1. At this moment it is unclear what the most preferred setup for an OMV based vaccine platform is. This section describes the impact of the heterologous antigen location, the endogenous loading of antigens to the vesicle lumen and the vesicle surface, and the exogenous loading of antigens to the vesicle lumen and the vesicle surface.

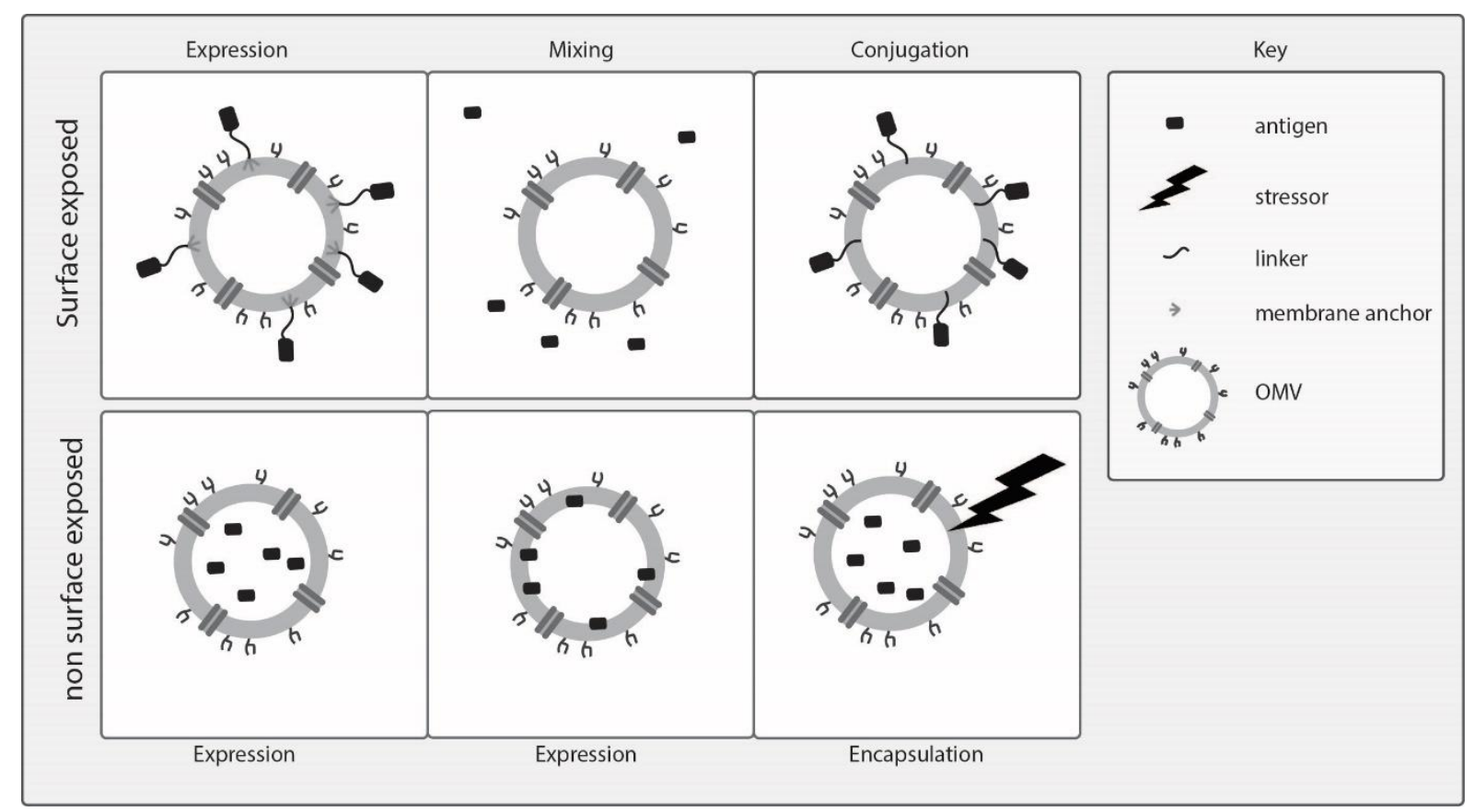

Figure 1. Methods of antigen decoration on OMVs. Top row shows surface exposed antigens on the vesicles, bottom row shows the antigens as luminal cargo of OMVs. Antigens can be produced by the OMV production bacterium (left), while antigen addition to purified vesicles can be divided in mixing, conjugation and encapsulation (middle and right).

Surface exposed antigens are accessible for antigen-specific B cell binding, while the inside of the vesicle is shielded from these cells. Luminal antigens may be skewed towards cytotoxic T-cell responses [15], hence the desired immune response determines the design of the OMV. Many groups have expressed antigens in the lumen of OMVs to develop OMV vaccines (Table 1) [16-21]. Surprisingly, these studies also find antibody-mediated immune responses against the luminal heterologous antigen. 
Muralinath et al. studied the luminal expression of Pneumococcal PspA in Salmonella enterica serovar Typhimurium OMVs [16]. These vesicles triggered minor antibody responses against OMVs and PspA in immunized mice and provided protection in a challenge experiment. OMVs without PspA or purified PspA alone did neither evoke an antibody response nor provide protection. Schild et al. showed minor specific antibody responses against periplasmic alkaline phosphatase (PhoA) from Escherichia coli expressed in Vibrio cholerae OMVs [17]. In addition to the minor antibody titers found in the previous two studies, it was shown by Fantappie et al. that also high functional antibody titers can be obtained by expressing heterologous antigens in their native conformation in the lumen of E. coli OMVs [18]. This systematic study characterized the vesicles by showing incorporation of the antigens and antigen localization. Because of the findings in a previous study that the Chlamydial HtrA protein expressed in OMVs was partially surface exposed, the authors checked the antigen localization (Box 1) by proteinase $\mathrm{K}$ treatment [20]. After all, contamination with surface exposed antigen could be a cause for the observed response. The heterologous antigens in OMVs were found not to be surface exposed. Further analysis of these proteins showed their native conformation in the vesicle lumen that remarkably appears to be sufficient to trigger antibody mediated responses.

Antigens can be presented on OMVs surface with exposure to the environment outside the OMV. We recently studied the expression of the Borrelial surface-exposed lipoprotein OspA in Neisseria meningitidis OMVs [1]. Expression of the protein in meningococci did not result in surface exposure on OMVs. To obtain surface exposure, OspA was fused to a Neisserial lipoprotein. The immunogenicity of this surface exposed fusion construct was compared to that of a luminal expressed OspA in mice. Results showed that only the surface-exposed OspA was able to elicit an OspA-specific antibody response. In a study on Salmonella OMVs by Muralinath et al., higher immune responses against outer membrane proteins and LPS were found than against the heterologous expressed antigen present in the vesicle lumen [16].

It remains unclear whether antigens in the lumen of OMVs provide sufficient antibody responses. The observed antibody responses of some studies may be biased by extracellular antigen or surface attached antigen. On the contrary the lack of an antigen specific antibody response against non-surface exposed OspA may not be predictive for 
other antigens [1]. Antibody responses have been observed for all surface exposed antigens, while for luminal antigens the provoked responses remain ambiguous. Altogether more research is required on the exact effect of the antigen location on efficacy of an OMV vaccine platform.

\section{Box 1: Analyzing the surface exposure of antigens on OMVs}

Analysis of the surface exposed protein expression is important, but challenging. Expression levels of the antigen can be assessed by immunostaining. The heterologous antigen expressed on the bacteria and on the OMV can be stained by a labeled antibody and detected by fluorescent microscopy [1]. Alternative options for the detection of immunostained bacteria or vesicles can be flow cytometry [5], ELISA or nanoparticle tracking analysis. Besides immunostaining, proteins associated to the outside of the outer membrane can be determined by proteinase K digestion of surface exposed proteins followed by SDS-PAGE analysis. Western Blotting can be used to test the proteinase K susceptibility of a specific antigen. This method has been used since the early 1980s for the detection of surface exposed proteins [8]. The protein profile of proteinase $\mathrm{K}$ treated vesicles can be compared to the protein profile of non-treated OMVs to detect proteinase $\mathrm{K}$ susceptibility of certain proteins. As a control, SDS can be added to the treatment to check the digestion of previously shielded proteins. 
Table 1.

Overview of heterologous antigens expressed on OMVs as vaccine concept.

\begin{tabular}{|c|c|c|c|c|c|c|c|}
\hline Type $^{1}$ & Antigen location & Antigen display & Antigen & Target & Backbone & Reference & Year \\
\hline sOMV & Surface exposed & ClyA fusion & Omp22 & Acinetobacter baumannii & Escherichia coli & {$[22]$} & 2016 \\
\hline sOMV & Surface exposed & ClyA fusion & M2e & Influenza A & Escherichia coli & {$[23]$} & 2016 \\
\hline $\begin{array}{l}\text { eOMV \& } \\
\text { sOMV }\end{array}$ & Surface exposed & fHbp fusion & OspA & Lyme's disease & Neisseria meningitidis & [1] (Intravacc) & 2016 \\
\hline glycOMV & Surface exposed & Lipid A glycan & F. tularensis O-PS & Francisella tularensis & Escherichia coli & {$[24]$} & 2016 \\
\hline geOMV & Surface exposed & Lipid A glycan & CPS14 & Pneumococcal disease & Escherichia coli & {$[25,26]$} & 2016 \\
\hline sOMV & Mixing & Mixing & AnAPN1, Pfs48/45 & Malaria & Escherichia coli & [27] & 2016 \\
\hline sOMV & Not determined & Hybrid flagellin FlaA & FliC & ETEC & Vibrio cholerae & {$[28]$} & 2015 \\
\hline sOMV & Surface exposed & Hbp fusion & PspA or Ply fragments & Pneumococcal disease & Salmonella typhimurium & [29] (Abera Biosciences) & 2015 \\
\hline sOMV & Surface exposed & Hbp fusion & $\begin{array}{l}\text { ESAT6, Ag85B fragments, } \\
\text { and Rv2660c }\end{array}$ & Tubercolosis & $\begin{array}{l}\text { Salmonella typhimurium/ } \\
\text { Escherichia coli }\end{array}$ & [30] (Abera Biosciences) & 2014 \\
\hline sOMV & Surface exposed & Hbp fusion & MOMP fragments & Chlamydia & Salmonella typhimurium & [30] (Abera Biosciences) & 2014 \\
\hline sOMV & OMV lumen & OmpA fusion & $\begin{array}{l}\text { SpyCEP, Streptolysin 0, } \\
\text { Spy0269 }\end{array}$ & $\begin{array}{l}\text { Group A Streptococcus } \\
\text { disease }\end{array}$ & Escherichia coli & [18] (Novartis) & 2014 \\
\hline sOMV & OMV lumen & OmpA fusion & SAM_1372 & $\begin{array}{l}\text { Group B Streptococcus } \\
\text { disease }\end{array}$ & Escherichia coli & [18] (Novartis) & 2014 \\
\hline sOMV & Mixing & Mixing & O-antigen & Shigellosis & Shigella spp. & {$[31,32]$} & 2013 \\
\hline
\end{tabular}




\begin{tabular}{|c|c|c|c|c|c|c|c|}
\hline sOMV & OMV lumen & OmpA fusion & HtrA & Chlamydia & Escherichia coli & {$[20,33]$ (Novartis) } & 2012 \\
\hline sOMV & Outer membrane & $\begin{array}{l}\text { OprI fusion } \\
\text { (Pseudomonas } \\
\text { aeruginosa) }\end{array}$ & A104R & African swine fever & Escherichia coli & {$[34]$} & 2012 \\
\hline sOMV & OMV lumen & $\begin{array}{l}\text { Type II secretion signal } \\
\text { from } \beta \text {-lactamase }\end{array}$ & PspA & Pneumococcal disease & $\begin{array}{l}\text { Salmonella enterica } \\
\text { (serovar Typhimurium }\end{array}$ & [16] & 2011 \\
\hline dOMV & Mixing & Mixing & glycoprotein D & Genital herpes & Neisseria meningitidis & {$[35,36]$} & 2010 \\
\hline sOMV & OMV lumen & OmpA fusion & FLAG & Proof of concept & Escherichia coli & {$[21]$} & 2009 \\
\hline sOMV & Surface exposed & AIDA fusion & KMP-11 & Leishmaniosis & Escherichia coli & [37] & 2009 \\
\hline sOMV & Vesicle lumen & Inherent & PhoA & Proof of concept & Vibrio cholerae & [17] & 2009 \\
\hline sOMV & Surface exposed & ClyA fusion & GFP & Proof of concept & Escherichia coli & {$[38,39]$} & 2008 \\
\hline dOMV & Mixing & mixing & $\begin{array}{l}\text { Dermatophagoides siboney } \\
\text { allergens }\end{array}$ & Dust mite allergy & Neisseria meningitidis & [40] & 2006 \\
\hline sOMV & Surface exposed & Inherent & NspA & Neisseria meningitidis & Neisseria flavescens & [41] & 2004 \\
\hline sOMV & Outer membrane & Inherent & Ail & Proof of concept & Escherichia coli & [19] & 2004 \\
\hline sOMV & OMV lumen & Tat signal & GFP & Proof of concept & Escherichia coli & [19] & 2004 \\
\hline
\end{tabular}

${ }^{1}$ See Box 2 for an explanation of the OMV types. 


\subsection{Endogenous loading of surface exposed antigens}

Recombinant expression of proteins in bacteria is a large and ever-growing field [42, 43]. Several approaches have been described to express proteins on the outer membrane of bacteria [44]. However, many of these approaches have a low yield or are only suited for small proteins or parts thereof. One method relies on antigenic proteins that are normally exported beyond the cell surface by proteolytic processing. These antigens can be retained by preventing the proteolysis [45]. Another method relies on targeting the protein to the outer membrane and thus to the OMVs [19]. One of the first concepts of outer membrane targeting for surface exposed antigen expression was based on autotransporters. Autotransporters are transported as unfolded protein over the inner membrane via the Sec pathway. Next, the autotransporter is transported over the outer membrane by a so far unknown mechanism that most likely requires direct involvement of the BamA protein [46]. The rightly folded autotransporter anchors to the outer membrane via a C-terminal $\beta$ domain. Fusion to the autotransporter hemoglobin protease ( $\mathrm{Hbp}$ ) of E. coli was used to express recombinant proteins in an attenuated strain of Salmonella typhimurium [47]. The authors engineered $\mathrm{Hbp}$ to facilitate the surface expression of Mycobacterium tuberculosis proteins and epitopes of the major outer membrane protein MOMP from Chlamydia thrachomatis [30]. This method of antigen display is highly efficient, however it seems limited to smaller protein fragments [48].

To express larger proteins on the surface of the outer membrane, Kim et al. fused several heterologous proteins including GFP with a 5 residue glycine linker to the Cterminus of the pore-forming cytotoxin ClyA [38]. These fusion constructs were efficiently transported across the inner membrane to the outer membrane of E. coli. Recently it was shown by their group that surface expressed M2e antigens on E. coli OMVs provided protection against influenza A infection in a mice study [23]. Besides single M2e-ClyA fusion constructs, the authors also successfully constructed a nearly 50 $\mathrm{kDa}$ multimeric variant containing the serine analogs of human, swine and two avian M2e variants. Apart from incorporation of a single protein on the OMV it was shown in a different study that a three-enzyme cascade could also be successfully engineered on $E$. coli OMVs [49]. The enzyme scaffold was expressed in the bacterium and anchored to the outer membrane by the truncated ice nucleation protein anchor [50]. The enzymes were produced separately from the OMV, which would allow the expression of different 
enzymes on the OMV by simple replacement of these enzymes for different proteins. The enzymes were attached to the protein anchor by using three cohesin-dockerin pairs. Cohesin domains interact with dockerin domains of enzymes to form multiprotein complexes [51]. To decorate the OMV with a three-enzyme cascade, the authors used the unique interaction between each of the three cohesin-dockerin pairs for the sequential attachment of the enzyme complex. The OMVs with the enzyme scaffold had a 23-fold higher conversion rate when compared to the individual enzymes free in solution [49]. Although this example is not related to vaccines it shows the potential of attaching proteins to the outside of the OMV.

Surface exposed expression of heterologous lipoproteins has been achieved by fusion of the heterologous lipoprotein to a membrane anchoring second lipoprotein. In this way, the heterologous lipoprotein signaling is not required to match the hosts signaling to achieve surface exposure. The borrelial lipoprotein OspA was fused via a tether to the $\mathrm{N}$-terminal part of factor $\mathrm{H}$ binding protein that acts as a membrane anchor [1]. The extracted OMV (eOMV) contained surface exposed OspA. With this approach it was possible to purify OMVs with surface exposed heterologous lipoprotein antigens.

\subsection{Endogenous loading of antigens to the OMV lumen}

Differences in protein composition of OMVs and the outer membrane have been described [52-54]. This difference hints towards a regulated protein sorting to OMVs or a regulated $\mathrm{OMV}$ release but the mechanism of protein enrichment remains unclear. Accumulation of misfolded and aggregated proteins in the periplasm is suggested as one of the possible mechanisms of vesicle release [13]. An increase in OMV formation was found in strains lacking the chaperone DegP, resulting in enrichment of misfolded DegP substrates in OMVs [55]. McBroom and Kuehn showed that it was possible to selectively enrich a protein in the OMV lumen by adding a misfolded outer membrane protein sequence to the periplasmic cytochrome $b_{562}$ [56]. A 12-fold enrichment of cytochrome b562 in OMVs was observed based on SDS-PAGE analysis. Although this method provides valuable insights in the method of protein sorting in OMVs, it may not be beneficial for the folding of the antigen. Thus, this method might not be preferred for the addition of antigens that require proper folding in the lumen of OMVs.

Antigens have been bioengineered to be targeted to the lumen of OMVs. Kesty and Kuehn fused GFP to the twin-arginine (Tat) signal sequence to produce E. coli OMVs 
with GFP in their lumen. This Tat pathway transfers folded proteins over the cytoplasmic membrane. To transfer unfolded proteins over the cytoplasmic membrane the Sec pathway could be used. However, GFP and some other proteins are unable to fold correctly in the periplasm. The OMVs containing GFP in their lumen showed to shield GFP from proteinases, which indicates the stability of proteins inside the vesicles [19]. Another method for luminal protein expression in E. coli OMVs is based on the fusion of proteins to the periplasmic side of the abundant outer membrane protein OmpA [21]. A FLAG-tag was attached to the truncated C-terminus of the OmpA protein, which is located at the periplasmic side of the outer membrane [21]. OmpA truncations or deletions are known to result in a blebbing phenotype. This approach combines stimulation of OMV production by the host with a site for antigen addition. This method was successfully used to express fusions of OmpA to several different antigens against Group A Streptococcus, Group B Streptococcus and Chlamydia [18, 20].

Another study has directed the pneumococcal protein PspA to the lumen of Salmonella typhimurium OMVs by fusion of PspA to the $\mathrm{N}$-terminal $\beta$-lactamase signal sequence [16]. This signal sequence allows the transport of $\beta$-lactamase to the periplasm by the type II secretion system, which yields OMVs with PspA in their lumen.

Taken together, heterologous antigens can be expressed in the lumen of OMVs by fusion of the antigen to secretion signals or periplasmic proteins. These fusion based methods are relatively straightforward and have been successfully used to express full size antigens in a conformational correct manner and antibodies have been found against these antigens. While it is unclear how the antigens are processed to eventually elicit antibody mediated immune responses, heterologous expression of antigens in the lumen of OMVs may be a feasible approach for an OMV vaccine platform.

\subsection{Exogenous loading of surface exposed antigens}

Antigens can be added afterwards by introducing affinity to the antigen on the OMV. This method of exogenous loading was used by adding a SpyTag to OmpA [57]. The SpyTag peptide is designed to form a covalent bond with the SpyCatcher protein to ease conjugation of proteins [58]. The SpyTag-OmpA fusion was expressed on OMVs and can be coupled to SpyCatcher fused to any protein. By this method, OMVs can be produced in mass production followed by adding antigens on the OMV. Similarly, this SpyTag based approach has been used to decorate VLPs with antigens as vaccine platform [59]. 


\subsection{Exogenous loading of antigens to the OMV lumen}

Loading of antigens to the vesicle lumen, after the vesicles and antigens have separately been produced in bulk, can be an attractive approach. The concentration of the active components may be better controllable when compared to endogenous loading. To load antigens in their native conformation to the OMV lumen in vitro, the vesicle should be opened and closed again without permanent damage. The exogenous loading of proteins to the vesicle lumen has not been described although several methods of loading smaller molecules into extracellular vesicles (EVs) are explored. EVs can be loaded by simple incubation (passive loading) or by active treatment of the EVs (active loading). Methods of loading EVs by electroporation, saponin-treatment, extrusion or dialysis are well described for EVs and even compared in a side by side study [60]. In this study different porphyrins were loaded in endothelial, stem cell, and cancer derived EVs. Active encapsulation techniques and especially saponin-treatment showed higher porphyrin loading than passive techniques. Active encapsulation by electroporation has been described to load small-molecule drugs and siRNA in to EVs (reviewed in [61]). Active encapsulation of siRNA in to E. coli OMVs by electroporation was applied by Gujrati et al. [62]. This siRNA targets kinesin spindle protein, which is upregulated in tumor and rapidly growing cells. The siRNA was electroporated to the lumen of the OMV resulting in intact siRNA-loaded vesicles. The loaded vesicle was targeted to HER2+ tumor cells by a HER2 antibody fused to ClyA. The authors were successful in reducing tumor growth in mice by treatment with these OMVs. For the loading of vesicles derived from the fungus Neurospora crassa, a single freeze-thaw method has been shown to be very effective [63]. Loading efficiencies of over $80 \%$ were reported and the authors claim the method is suitable for any protein. Interestingly, this method has to our knowledge not been tested for bacterial OMVs.

\subsection{Mixing OMVs}

It is currently unknown whether antigens require attachment to the vesicles. It has been shown that for Adjuvant System 03, a marketed oil in water adjuvant, it is sufficient to inject the antigen at the same location as the adjuvant to obtain an adjuvating effect [64]. In this case, simple mixing of the adjuvant and antigen is sufficient. The mixing of the weak antigen GFP with E. coli OMVs showed however, that immunized mice did not elicit anti-GFP IgG responses [39]. Attachment of GFP to the OMVs showed enhanced responses, comparable to GFP absorbed to the commercial standard adjuvant alum. If 
attachment is desired, the antigens could be conjugated to the OMV. This has been successfully accomplished for Brucella LPS that was conjugated to N. meningitidis OMVs [65]. Along the same line capsular polysaccharides from serogroup C meningococci were conjugated to a $N$. meningitidis serogroup B OMV [66]. Using conjugation, mixing, heterologous expression, or a combination thereof, also multivalent vaccines can be designed using OMVs as a main component. A combination of mixing and recombinant antigen expression has been applied for the eOMV based N. meningitidis serogroup B concept vaccines Hexamen and Nonamen $[67,68]$. Mixing dOMVs from two strains has been applied to produce a combined $N$. meningitidis serogroup A and W concept vaccine [69]. Mixing OMVs has been applied by a recently proposed sOMV based Shigellosis concept vaccine [31]. The authors of this latter study produced sOMV from 6 serogroups of Shigellae that were mixed to form a hexavalent vaccine. This composition was named MOMV (not to be mistaken with modified OMVs or the previous naming of mitochondrial EVs, see box 2 for naming of different types of OMVs) because of the multi-serotype OMV composition [31]. Roier et al. created OMV mixtures of nontypeable H. influenzae strains to obtain a broad covering immunization mixture [70]. The OMV mixture showed a broadly covering protective immune response, although it should be noted that OMVs derived from a single strain also showed cross-protection against other nontypeable $H$. influenzae strains. Mixing of OMVs from enterotoxigenic E. coli and $V$. cholerae was described by Leitner and coworkers [28]. The authors showed an improved protection for the mixed OMVs when compared to the provoked immune response from the individual OMVs alone. Mixing of OMVs can thus be a simple yet very effective method of improving protection.

\section{Designing the OMV: Bioengineering the immune response}

The interaction of the OMV with the immune system can be considerably tuned. Outer membrane vesicles can be directly used as a vaccine, since the vesicles are nonreplicating and contain antigens as well as PAMPs in a particle configuration. Furthermore, OMVs can induce both humoral and cellular immune responses [12]. Recently it was shown by Rosenthal et al. that E. coli OMVs can trigger a Th1-biased immune response [71]. The authors were able to induce both protective humoral and Th1-biased cellular immune responses against the heterologous antigen by using the probiotic E. coli Nissle 1917 bacteria as OMV production host. This probiotic bacterium itself is highly immunosuppressive, and thus not an obvious vaccine candidate, 
nevertheless its OMVs lack this capacity [72]. In contrast to the OMVs from E. coli K12, the probiotic derived OMVs elicit Th1 cellular immune responses, however these responses were not necessarily directed against the heterologous expressed antigens [71]. To improve the immune response against an antigen of interest, the antigen amount per OMV can be increased or multiple antigens can be included. Zhang and coworkers improved the immunogenicity of a trivalent $N$. meningitidis vaccine by genetic engineering a second fHbp protein in the $\operatorname{por} B$ gene, which increased fHbp yields in the OMV and increased antibody responses against fHbp [73].

\section{Box 2: Naming of OMVs}

With the production of extracted OMVs, many prefixes have been used to clarify the origin of the OMVs [2]. The naming of the different types of vesicles has been based on the method of extraction, for example, the detergent deoxycholate (DOC) has been used to extract OMVs from bacteria to develop the first OMV-based vaccines [3]. This harsh extraction method was used to decrease the toxic LPS in the vesicles and the produced vesicles were referred to as detergent OMVs (dOMVs). The use of prefixes in OMV naming starts to become confusing due to the development of more and more methods of extracting vesicles and methods of increased "spontaneous" release of vesicles in the culture supernatant. The term nOMV has been used for both natural OMVs and native OMVs, where native OMVs were extracted by EDTA. It was shown that EDTA-extracted OMVs were similar to natural OMVs and hence the term nOMVs or N-OMVs was used [4]. To distinguish between spontaneous and extracted vesicles we favor to refer to EDTA extracted vesicles as extracted OMV: eOMV. Moreover, spontaneously released OMV in vivo should be distinguished from OMV released in vitro. The in vitro production of spontaneous released OMV during cultivations is known to be induced by many mutations or stressors. The possible difference between these stress released vesicles and vesicles released spontaneously by the bacterium are more difficult to observe and researchers should be careful when comparing differently produced OMV. We suggest to refer to in vitro produced OMVs as spontaneous released OMVs: sOMVs. Overall it has become challenging to apply a uniform naming system of OMVs and thus it should be cared for that the method of producing OMVs is accurately described. We propose to uniform the naming of OMVs as listed in Table 2. 
OMVs can be engineered such that the vesicles contain proteins altered in their interaction with human receptors. Opacity (Opa) proteins of Neisseria sp. are known to interact with human CEACAM receptors [74]. This interaction causes a reduced Opaspecific immune response, however the immune response against the other OMV antigens was not significantly affected [75]. Another hypothesis is that binding of OMV proteins by human complement inhibitors could interfere with the development of the immune response. A reduction in antibody responses was shown due to binding of the antigen with a human protein [76]. Human factor $\mathrm{H}$ could be bound by Neisserial fHbp, NspA and Porin B2. Especially the interaction with fHbp is studied widely due to the recent licensing of two fHbp based $N$. meningitidis serogroup B vaccines $[77,78]$. It was shown that partial inhibition of $\mathrm{fH}$ binding did not enhance the immunity in a mouse model [79]. Human Factor H transgenic mice were immunized with low fH binding fHbp and showed to elicit higher serum bactericidal antibody responses than when immunized with fHbp capable of binding fH [80]. Furthermore, immunizations with a low $\mathrm{fH}$ binding recombinant $\mathrm{fHbp}$ in an infant rhesus macaques model showed antibody responses directed to the region of fHbp that binds human factor $\mathrm{H}$ [81]. Antibody responses against these host protein binding antigens can be improved by low-binding mutants, although these show no alteration in the antibody responses against other proteins present on the OMV.

Table 2. Overview of the proposed naming of OMVs

\begin{tabular}{l|l}
\hline Abbreviation & $\begin{array}{l}\text { Type of OMV } \\
\text { dOMV }\end{array}$ \\
mOMV & $\begin{array}{l}\text { Detergent extracted OMVs from in vitro cultured bacteria } \\
\text { All non-detergent extracted OMV from in vitro cultured bacteria } \\
\text { Preferably not to be used due to its ambiguity (modified OMV, } \\
\text { multivalent OMV, mitochondrial OMV) } \\
\text { nOMV }\end{array}$ \\
SOMV & $\begin{array}{l}\text { Sreferably not to be used due to its ambiguity (native OMV or } \\
\text { natural OMV) } \\
\text { Spontaneous released OMV, typically obtained from the }\end{array}$ \\
\end{tabular}


OMVs contain by nature many immunogenic components that could cause a reaction against the OMV backbone. Currently it is unknown whether this reaction causes undesired effects. The possibility of this effect affects the choice of backbones (Table 1). The use of $E$. coli as backbone has the advantage of being extensively studied $[18,19$, $21-25,33,34,37,38,82]$, however the immune response directed against $E$. coli could negatively influence commensal populations. This negative effect could be prevented by the use of a pathogenic bacterium as backbone. Pathogenic backbones as $N$.

meningitidis, S. typhimurium and V. cholerae have been described $[1,17,30]$, although it is unknown what the effect is on the spread of the production pathogen itself. Another aspect is the possible response against the backbone during the repetitive use of the OMV platform against different diseases. Alternatively, a stealth backbone could be developed. Cross reactivity of the backbone with closely related species was described for $N$. meningitidis $[83,84]$ illustrating the possible broader effect of immune responses against the OMV backbone. Currently it is unknown to what extent minor crossreactivity impacts a vaccine platform or if a complete stealth OMV backbone is required.

\section{Designing the OMV: Bioengineering toxicity and adverse effects}

As a very potent activator of the immune system, LPS can induce severe side effects and must be detoxified. Detoxification by reducing the amount of LPS has been performed by a detergent extraction process yielding dOMV [85]. To use native or spontaneous OMVs, most vaccine concepts are based on genetically detoxified LPS, with potent activation of the innate immune system by TLR4. The TLR4 activation can be altered by bioengineering the LPS [86], since too potent TLR4 reactivity causes endotoxicity [87]. The endotoxin activity of LPS was modified by $m s b A$ deletion in E. coli or IpxL1 deletion in N. meningitidis, resulting in lipid A without the secondary acyl chain [88, 89]. These modifications strongly alter the endotoxicity by reducing the TLR4/MD-2 activation by the LPS. Further bioengineering of $N$. meningitidis LPS showed that a broad range of TLR4 activation can be obtained [86]. Furthermore, a broad range of differential cytokine inducing properties was observed by the different bioengineered LPS species. LPS bioengineering to reduce LPS reactivity has been applied on many different species [90]. Besides OMVs from bioengineered N. meningitidis, vesicles with genetically detoxified LPS have also been produced from $E$. coli and $V$. cholarae $[28,91]$. Recently, $E$. coli OMVs containing only the lipid IVa of LPS instead of the full LPS have been produced 
[92]. These OMVs retained sufficient capabilities of eliciting immune responses against the included heterologous antigens.

Besides targeted attempts to alter the endotoxicity of the OMV, it is important to take into account that the production method has severe impact on the endotoxicity. It was shown that different OMVs (sOMV, eOMV and dOMV, see Box 2) derived from the same strain have differences in TLR4 and TLR2 activation [93]. To improve the OMV productivity of $H$. pylori, Tol-Pal knockouts were constructed [94]. However, the downside of the increased vesicle production was the more potent induction of inflammation of these OMVs, by significantly higher IL-8 levels, in host cells than wildtype OMVs. This indicates once more the complexity involved in the design of an OMV based vaccine.

Recently a novel type of vaccine platform based on OMVs was described by two groups, based on heterologous expressed glycan antigens instead of antigenic proteins [24-26]. These glyco-engineered OMVs (geOMVs) designed by Price et al. are based on the similarity between the polysaccharide translocation in the expression host and the vaccine target [25]. The capsular polysaccharide of $S$. pneumoniae was expressed in an E. coli strain lacking its own 0-antigen yielding attachment of the S. pneumoniae capsular polysaccharides to the E. coli lipid A core. In another study, Chen et al. produced glycosylated OMVs that provided protection against $F$. tularensis challenge in mice, by expression recombinant O-antigen polysaccharide on E. coli OMVs [24]. Furthermore, several lipid A modifications were made resulting in lowered TLR4 activation similarly to the attempts of detoxifying OMV associated LPS. The ease of adding heterologous glycans on OMVs emphasizes the potential of an OMV based vaccine platform for polysaccharide vaccines.

\section{Designing the OMV: physiochemical properties}

The size of OMVs is an overlooked part of OMV vaccine research that is, however, very relevant since the size influences the uptake by antigen presenting cells (APCs). Macrophages preferentially take up larger particles (Figure 2) where dendritic cells (DCs) take up particles in the size range of OMVs and VLPs [95]. OMVs range in size from 20-250nm in diameter [13] and size is assumed to be homogeneous and similar between different bacterial species. However, minor differences are reported in the size distributions of SOMV, eOMV and dOMV from the same bacterium in similar production 
processes [4]. The size of OMVs is important for the design of the production process [96]. For example, sterile filtration is only possible for smaller sized OMVs. Moreover, a minimum size of OMVs is required for adequate analysis of OMV quality. Conventional flow cytometry, for example, is not suitable for the analysis of particles smaller than 300 $\mathrm{nm}$ [97]. Improvements on flow cytometry have been made improving the detection limit [98] and methods focused on nanoparticles, such as Nanoparticle Tracking Analysis (NTA)[99] and Tunable Resistive Pulse Sensing [100], have been developed. With these improved methods a size limit of around $20 \mathrm{~nm}$ remains for appropriate detection of nanoparticles.

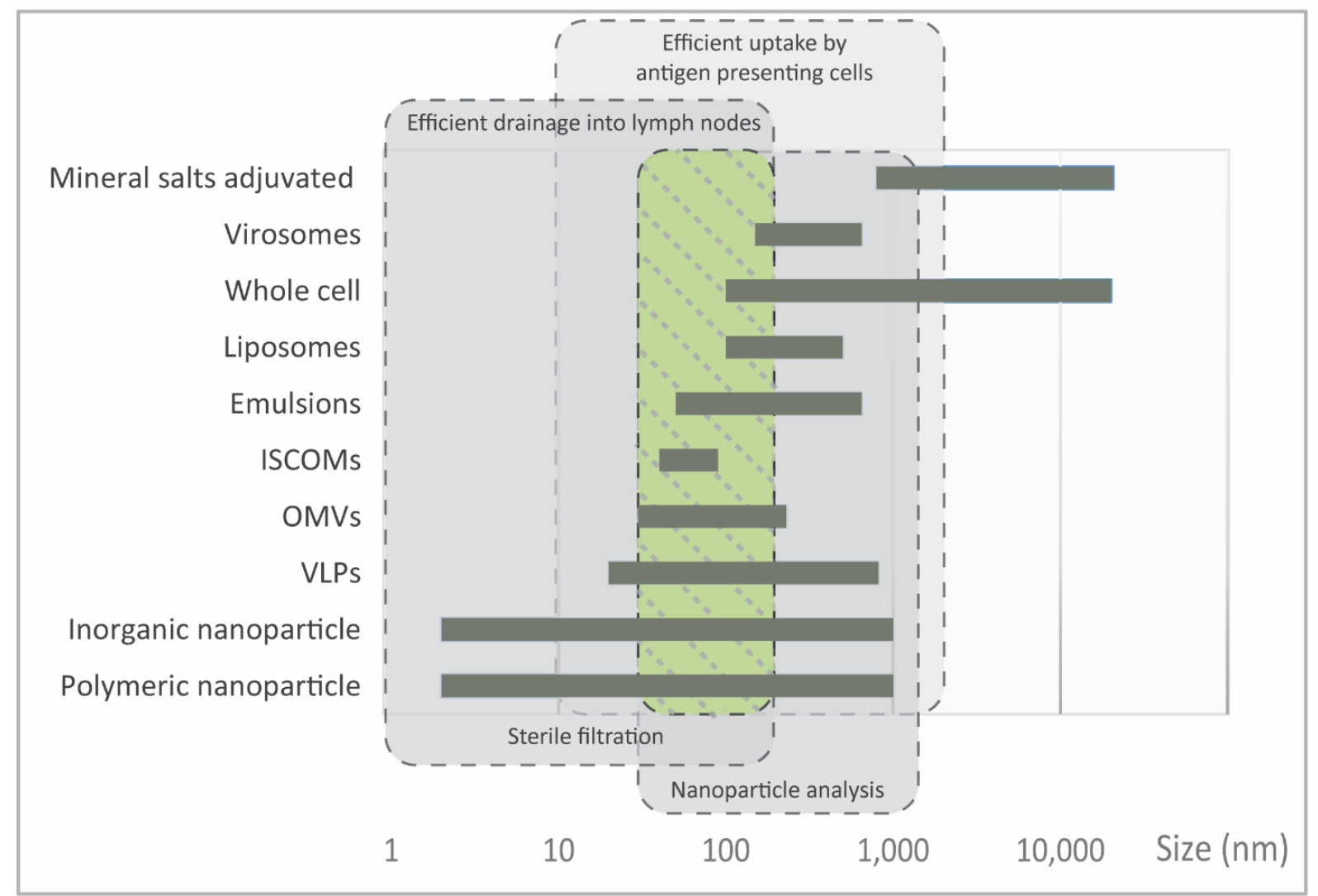

Figure 2. The size of vaccine concepts and the required size range for accessible production compared to the interaction with the immune system. The possibility of sterile filtration simplifies the design of the production process, while the analysis of nanoparticle size and the number of nanoparticles is essential for quality control. The green box highlights the overall preferred size window for vaccine production.

The size and shape are important parameters in the field of synthetic nanoparticles, and these physiochemical properties are highly tunable. Recently Gao and co-workers made an interesting combination of bacterial outer membrane vesicles and synthetic gold nanoparticles (AuNPs) [101]. OMVs were coated with mechanical force on gold 
nanoparticles to produce $30 \mathrm{~nm}$ sized membrane-coated AuNPs. Mice vaccinated subcutaneously with these nanoparticles showed rapid activation of DCs in lymph nodes, while vaccination with $90 \mathrm{~nm}$ sized membrane coated nanoparticles showed reduced accumulation of DCs. The membrane coated AuNPs of $30 \mathrm{~nm}$ showed higher induction of specific antibody responses, IFN-gamma production, and IL-17 production when compared to OMVs alone. The IL-4 production was low for all groups indicating a strong Th1- and Th17-biased cellular response. Another study compared ovalbumin loaded styrene nanoparticles with different size and shape, concluding that the smallest (193 nm) spherical particles elicit the strongest Th1 and Th2 immune responses [102]. This smaller spherical particle showed a Th1-biased response while the larger (1530 $\mathrm{nm}$ ) rod-shaped particle elicits a Th2-biased immune response. Smaller nanoparticles $(10-200 \mathrm{~nm})$ are able to drain freely to the lymph nodes, while larger nanoparticles $(500 \mathrm{~nm}-2000 \mathrm{~nm})$ require dendritic cells [103]. The larger particles are thus unable to target cells residing in the lymph nodes. Size of the vesicles is thus an important parameter in navigating the immune response by the efficient uptake by antigen presenting cells and efficient entry in to lymph nodes.

\section{Concluding Remarks and Future Perspectives}

OMVs have been proven to be a flexible vaccine production platform. Heterologous proteins and glycan antigens can be easily added to the vesicle, the immune stimulating properties of the vesicle can be engineered, and the toxicity can be reduced. Future research should address the immune response against the platform backbone, the heterogeneity of the vesicle product and the relatively underexplored effects of luminal OMV components. Besides the use of OMVs as prophylactic vaccines, vast possibilities of OMVs as therapeutic vaccines exist. With the approval of human use of several OMV based meningococcal vaccines, the future of OMV vaccines is bright, paving the way to designer and tailor made OMV vaccines. The use of OMV based vaccine platforms will facilitate enhanced vaccine design and will speed up the introduction of new much needed vaccines. 


\section{References}

1. Salverda, M.L., et al., Surface display of a borrelial lipoprotein on meningococcal outer membrane vesicles. Vaccine, 2016. 34(8): p. 1025-33.

2. van der Pol, L., M. Stork, and P. van der Ley, Outer membrane vesicles as platform vaccine technology. Biotechnology Journal, 2015. 10(11): p. 1689-1706.

3. Sierra, G.V., et al., Vaccine against group B Neisseria meningitidis: protection trial and mass vaccination results in Cuba. NIPH Ann, 1991. 14(2): p. 195-207; discussion 208-10.

4. van de Waterbeemd, B., et al., Improved OMV vaccine against Neisseria meningitidis using genetically engineered strains and a detergent-free purification process. Vaccine, 2010. 28(30): p. 4810-6.

5. Rioux, S., et al., Localization of surface immunogenic protein on group B streptococcus. Infect Immun, 2001. 69(8): p. 5162-5.

6. Rappuoli, R., From Pasteur to genomics: progress and challenges in infectious diseases. Nat Med, 2004. 10(11): p. 1177-1185.

7. WHO, W.H.O., Antimicrobial resistance: global report on surveillance. WHO press, 2014.

8. Freudl, R., et al., Cell surface exposure of the outer membrane protein OmpA of Escherichia coli K-12. J Mol Biol, 1986. 188(3): p. 491-4.

9. Kushnir, N., S.J. Streatfield, and V. Yusibov, Virus-like particles as a highly efficient vaccine platform: diversity of targets and production systems and advances in clinical development. Vaccine, 2012. 31(1): p. 58-83.

10. Zhao, L., et al., Nanoparticle vaccines. Vaccine, 2014. 32(3): p. 327-337.

11. Singh, M., A. Chakrapani, and D. O'Hagan, Nanoparticles and microparticles as vaccine-delivery systems. Expert Review of Vaccines, 2007. 6(5): p. 797-808.

12. Ellis, T.N. and M.J. Kuehn, Virulence and immunomodulatory roles of bacterial outer membrane vesicles. Microbiol Mol Biol Rev, 2010. 74(1): p. 81-94.

13. Kulp, A. and M.J. Kuehn, Biological functions and biogenesis of secreted bacterial outer membrane vesicles. Annu Rev Microbiol, 2010. 64: p. 163-84.

14. Arigita, C., et al., Stability of mono- and trivalent meningococcal outer membrane vesicle vaccines. Vaccine, 2004. 22(5-6): p. 629-42.

15. Galen, J.E. and R. Curtiss 3rd, The delicate balance in genetically engineering live vaccines. Vaccine, 2014. 32(35): p. 4376-4385.

16. Muralinath, M., et al., Immunization with Salmonella enterica serovar Typhimurium-derived outer membrane vesicles delivering the pneumococcal protein PspA confers protection against challenge with Streptococcus pneumoniae. Infect Immun, 2011. 79(2): p. 887-94.

17. Schild, S., et al., Characterization of Vibrio cholerae outer membrane vesicles as a candidate vaccine for cholera. Infect Immun, 2009. 77(1): p. 472-84

18. Fantappie, L., et al., Antibody-mediated immunity induced by engineered Escherichia coli OMVs carrying heterologous antigens in their lumen. J Extracell Vesicles, 2014. 3.

19. Kesty, N.C. and M.J. Kuehn, Incorporation of heterologous outer membrane and periplasmic proteins into Escherichia coli outer membrane vesicles. J Biol Chem, 2004. 279(3): p. 2069-76.

20. Bartolini, E., et al., Recombinant outer membrane vesicles carrying Chlamydia muridarum HtrA induce antibodies that neutralize chlamydial infection in vitro. J Extracell Vesicles, 2013. 2.

21. Kim, S.H., et al., Structural modifications of outer membrane vesicles to refine them as vaccine delivery vehicles. Biochim Biophys Acta, 2009. 1788(10): p. 2150-9.

22. Huang, W., et al., Employing Escherichia coli-derived outer membrane vesicles as an antigen delivery platform elicits protective immunity against Acinetobacter baumannii infection. Sci Rep, 2016. 6: p. 37242.

23. Rappazzo, C.G., et al., Recombinant M2e outer membrane vesicle vaccines protect against lethal influenza A challenge in BALB/c mice. Vaccine, 2016. 34(10): p. 1252-1258.

24. Chen, L., et al., Outer membrane vesicles displaying engineered glycotopes elicit protective antibodies. Proc Natl Acad Sci U S A, 2016. 113(26): p. E3609-18.

25. Price, N.L., et al., Glycoengineered Outer Membrane Vesicles: A Novel Platform for Bacterial Vaccines. Scientific Reports, 2016. 6: p. 24931.

26. Feldman, M., et al., Glycoengineered outer membrane vesicles and use thereof as vaccines. 2013, The governors of the Univeristy of Alberta.

27. Pritsch, M., et al., Comparison of Intranasal Outer Membrane Vesicles with Cholera Toxin and Injected MF59C.1 as Adjuvants for Malaria Transmission Blocking Antigens AnAPN1 and Pfs48/45. J Immunol Res, 2016. 2016: p. 3576028. 
28. Leitner, D.R., et al., A combined vaccine approach against Vibrio cholerae and ETEC based on outer membrane vesicles. Front Microbiol, 2015. 6: p. 823.

29. Kuipers, K., et al., Salmonella outer membrane vesicles displaying high densities of pneumococcal antigen at the surface offer protection against colonization. Vaccine, 2015. 33(17): p. 2022-9.

30. Daleke-Schermerhorn, M.H., et al., Decoration of outer membrane vesicles with multiple antigens by using an autotransporter approach. Appl Environ Microbiol, 2014. 80(18): p. 5854-65.

31. Mitra, S., M.K. Chakrabarti, and H. Koley, Multi-serotype outer membrane vesicles of Shigellae confer passive protection to the neonatal mice against shigellosis. Vaccine, 2013. 31(31): p. 3163-73.

32. Koley, H., et al., A multi-serotype outer membrane vesicles (MOMV) of Shigellae as a novel candidate vaccine. 2014, Indian Council Of Medical Research.

33. Bartolini, E., et al., Omv (outer membrane vesicle) vaccines. 2012, Novartis AG.

34. Basto, A.P., et al., A new cloning system based on the Oprl lipoprotein for the production of recombinant bacterial cell wall-derived immunogenic formulations. J Biotechnol, 2012. 157(1): p. 50-63.

35. Del Campo, J., et al., Intranasal immunization with a proteoliposome-derived cochleate containing recombinant gD protein confers protective immunity against genital herpes in mice. Vaccine, 2010. 28(5): p. 1193-200.

36. Romeu, B., et al., Combined meningococcal serogroup A and W135 outer-membrane vesicles activate cell-mediated immunity and long-term memory responses against non-covalent capsular polysaccharide A. Immunol Res, 2014. 58(1): p. 75-85.

37. Schroeder, J. and T. Aebischer, Recombinant outer membrane vesicles to augment antigen-specific live vaccine responses. Vaccine, 2009. 27(48): p. 6748-54.

38. Kim, J.Y., et al., Engineered bacterial outer membrane vesicles with enhanced functionality. J Mol Biol, 2008. 380(1): p. 51-66.

39. Chen, D.J., et al., Delivery of foreign antigens by engineered outer membrane vesicle vaccines. Proc Natl Acad Sci U S A, 2010. 107(7): p. 3099-104.

40. Lastre, M., et al., Bacterial derived proteoliposome for allergy vaccines. Vaccine, 2006. 24 Suppl 2: p. S2-34-5.

41. O'Dwyer C, A., et al., Expression of heterologous antigens in commensal Neisseria spp.: preservation of conformational epitopes with vaccine potential. Infect Immun, 2004. 72(11): p. 6511-8.

42. Baneyx, F. and M. Mujacic, Recombinant protein folding and misfolding in Escherichia coli. Nat Biotech, 2004. 22(11): p. 1399-1408.

43. Rosano, G.L. and E.A. Ceccarelli, Recombinant protein expression in Escherichia coli: advances and challenges. Frontiers in Microbiology, 2014. 5: p. 172.

44. van Bloois, E., et al., Decorating microbes: surface display of proteins on Escherichia coli. Trends Biotechnol, 2011. 29(2): p. 79-86.

45. Georgiou, G. and L. Segatori, Preparative expression of secreted proteins in bacteria: status report and future prospects. Curr Opin Biotechnol, 2005. 16(5): p. 538-45.

46. Sauri, A., et al., The Bam (Omp85) complex is involved in secretion of the autotransporter haemoglobin protease. Microbiology, 2009. 155(Pt 12): p. 3982-91.

47. Jong, W.S.P., et al., An autotransporter display platform for the development of multivalent recombinant bacterial vector vaccines. Microbial Cell Factories, 2014. 13: p. 162.

48. Luirink, J. and W.S.P. Jong, Fusion protein for secretory protein expression. 2016, Abera Bioscience AB.

49. Park, M., et al., Positional assembly of enzymes on bacterial outer membrane vesicles for cascade reactions. PLoS One, 2014. 9(5): p. e97103.

50. Shimazu, M., et al., Cell surface display of organophosphorus hydrolase in Pseudomonas putida using an ice-nucleation protein anchor. Biotechnol Prog, 2003. 19(5): p. 1612-4.

51. Carvalho, A.L., et al., Cellulosome assembly revealed by the crystal structure of the cohesin-dockerin complex. Proc Natl Acad Sci U S A, 2003. 100(24): p. 13809-14.

52. Lappann, M., et al., Comparative proteome analysis of spontaneous outer membrane vesicles and purified outer membranes of Neisseria meningitidis. J Bacteriol, 2013. 195(19): p. 4425-35.

53. Haurat, M.F., et al., Selective sorting of cargo proteins into bacterial membrane vesicles. J Biol Chem, 2011. 286(2): p. 1269-76.

54. Veith, P.D., et al., Porphyromonas gingivalis Outer Membrane Vesicles Exclusively Contain Outer Membrane and Periplasmic Proteins and Carry a Cargo Enriched with Virulence Factors. Journal of Proteome Research, 2014. 13(5): p. 2420-2432.

55. Schwechheimer, C. and M.J. Kuehn, Synthetic effect between envelope stress and lack of outer membrane vesicle production in Escherichia coli. J Bacteriol, 2013. 195(18): p. 4161-73.

56. McBroom, A.J. and M.J. Kuehn, Release of outer membrane vesicles by Gram-negative bacteria is a novel envelope stress response. Mol Microbiol, 2007. 63(2): p. 545-58. 
57. Alves, N.J., et al., Bacterial Nanobioreactors--Directing Enzyme Packaging into Bacterial Outer Membrane Vesicles. ACS Appl Mater Interfaces, 2015. 7(44): p. 24963-72.

58. Zakeri, B., et al., Peptide tag forming a rapid covalent bond to a protein, through engineering a bacterial adhesin. Proc Natl Acad Sci U S A, 2012. 109(12): p. E690-7.

59. Brune, K.D., et al., Plug-and-Display: decoration of Virus-Like Particles via isopeptide bonds for modular immunization. Sci Rep, 2016. 6: p. 19234.

60. Fuhrmann, G., et al., Active loading into extracellular vesicles significantly improves the cellular uptake and photodynamic effect of porphyrins. Journal of Controlled Release, 2015. 205: p. 35-44.

61. Lamichhane, T.N., R.S. Raiker, and S.M. Jay, Exogenous DNA Loading into Extracellular Vesicles via Electroporation is Size-Dependent and Enables Limited Gene Delivery. Mol Pharm, 2015. 12(10): p. 3650-7.

62. Gujrati, V., et al., Bioengineered bacterial outer membrane vesicles as cell-specific drug-delivery vehicles for cancer therapy. ACS Nano, 2014. 8(2): p. 1525-37.

63. Mayer, A., et al., Purified and protein-loaded mitochondrial outer membrane vesicles for functional analysis of preprotein transport. Methods Enzymol, 1995. 260: p. 252-63.

64. Morel, S., et al., Adjuvant System AS03 containing $\alpha$-tocopherol modulates innate immune response and leads to improved adaptive immunity. Vaccine, 2011. 29(13): p. 2461-2473.

65. Siadat, S.D., et al., Preparation and Evaluation of a New Lipopolysaccharide-based Conjugate as a Vaccine Candidate for Brucellosis. Osong Public Health and Research Perspectives, 2015. 6(1): p. 9-13.

66. Fukasawa, L.O., et al., Optimization of the conjugation method for a serogroup B/C meningococcal vaccine. Biotechnol Appl Biochem, 2006. 45(Pt 3): p. 141-6.

67. Claassen, I., et al., Production, characterization and control of a Neisseria meningitidis hexavalent class 1 outer membrane protein containing vesicle vaccine. Vaccine, 1996. 14(10): p. 1001-1008.

68. Kaaijk, P., et al., Preclinical safety and immunogenicity evaluation of a nonavalent PorA native outer membrane vesicle vaccine against serogroup B meningococcal disease. Vaccine, 2013. 31(7): p. 106571.

69. Tunheim, G., et al., Preclinical immunogenicity and functional activity studies of an A+W meningococcal outer membrane vesicle (OMV) vaccine and comparisons with existing meningococcal conjugate- and polysaccharide vaccines. Vaccine, 2013. 31(51): p. 6097-106.

70. Roier, S., et al., Intranasal Immunization with Nontypeable Haemophilus influenzae Outer Membrane Vesicles Induces Cross-Protective Immunity in Mice. PLoS ONE, 2012. 7(8): p. e42664.

71. Rosenthal, J.A., et al., Mechanistic insight into the TH1-biased immune response to recombinant subunit vaccines delivered by probiotic bacteria-derived outer membrane vesicles. PLoS One, 2014. 9(11): p. e112802.

72. Kim, O.Y., et al., Immunization with Escherichia coli outer membrane vesicles protects bacteria-induced lethality via Th1 and Th17 cell responses. J Immunol, 2013. 190(8): p. 4092-102.

73. Zhang, L., et al., Improving the immunogenicity of a trivalent Neisseria meningitidis native outer membrane vesicle vaccine by genetic modification. Vaccine, 2016. 34(35): p. 4250-4256.

74. Billker, 0., et al., The structural basis of CEACAM-receptor targeting by neisserial Opa proteins. Trends Microbiol, 2000. 8(6): p. 258-260.

75. Zariri, A., et al., Expression of human CEACAM1 in transgenic mice limits the Opa-specific immune response against meningococcal outer membrane vesicles. Vaccine, 2013. 31(47): p. 5585-93.

76. Costa, I., R. Pajon, and D.M. Granoff, Human factor H (FH) impairs protective meningococcal anti-FHbp antibody responses and the antibodies enhance FH binding. MBio, 2014. 5(5): p. e01625-14.

77. Serruto, D., et al., The new multicomponent vaccine against meningococcal serogroup B, 4CMenB: immunological, functional and structural characterization of the antigens. Vaccine, 2012. 30 Suppl 2: p. B87-97.

78. Marshall, H.S., et al., A phase 2 open-label safety and immunogenicity study of a meningococcal B bivalent rLP2086 vaccine in healthy adults. Vaccine, 2013. 31(12): p. 1569-1575.

79. Daniels-Treffandier, H., et al., Impact of Reducing Complement Inhibitor Binding on the Immunogenicity of Native Neisseria meningitidis Outer Membrane Vesicles. PLoS One, 2016. 11(2): p. e0148840.

80. Rossi, R., D.M. Granoff, and P.T. Beernink, Meningococcal factor H-binding protein vaccines with decreased binding to human complement factor $\mathrm{H}$ have enhanced immunogenicity in human factor $\mathrm{H}$ transgenic mice. Vaccine, 2013. 31(46): p. 5451-7.

81. Granoff, D.M., et al., Enhanced protective antibody to a mutant meningococcal factor H-binding protein with low-factor H binding. JCI Insight, 2016. 1(14): p. e88907.

82. Pritsch, M., et al., Comparison of Intranasal Outer Membrane Vesicles with Cholera Toxin and Injected MF59C.1 as Adjuvants for Malaria Transmission Blocking Antigens AnAPN1 and Pfs48/45. Journal of Immunology Research, 2016. 2016: p. 11. 
83. Sanchez, S., et al., Evaluation of cross-reactive antigens as determinants of cross-bactericidal activity in pathogenic and commensal Neisseria. Vaccine, 2001. 19(25-26): p. 3390-8.

84. Oliver, K.J., et al., Neisseria lactamica protects against experimental meningococcal infection. Infect Immun, 2002. 70(7): p. 3621-6.

85. Zollinger, W.D., et al., Safety and immunogenicity of a Neisseria meningitidis type 2 protein vaccine in animals and humans. J Infect Dis, 1978. 137(6): p. 728-39.

86. Zariri, A., et al., Modulating endotoxin activity by combinatorial bioengineering of meningococcal lipopolysaccharide. Sci Rep, 2016. 6: p. 36575.

87. Fitzgerald, K.A., D.C. Rowe, and D.T. Golenbock, Endotoxin recognition and signal transduction by the TLR4/MD2-complex. Microbes Infect, 2004. 6(15): p. 1361-7.

88. Somerville, J.E., Jr., et al., A novel Escherichia coli lipid A mutant that produces an antiinflammatory lipopolysaccharide. J Clin Invest, 1996. 97(2): p. 359-65.

89. van der Ley, P., et al., Modification of lipid A biosynthesis in Neisseria meningitidis lpxL mutants: influence on lipopolysaccharide structure, toxicity, and adjuvant activity. Infect Immun, 2001. 69(10): p. 5981-90.

90. Zariri, A. and P. van der Ley, Biosynthetically engineered lipopolysaccharide as vaccine adjuvant. Expert Rev Vaccines, 2015. 14(6): p. 861-76.

91. Leitner, D.R., et al., Lipopolysaccharide modifications of a cholera vaccine candidate based on outer membrane vesicles reduce endotoxicity and reveal the major protective antigen. Infect Immun, 2013. 81(7): p. 2379-93.

92. Watkins, H.C., et al., Safe Recombinant Outer Membrane Vesicles that Display M2e Elicit Heterologous Influenza Protection. Molecular Therapy, 2017. 25(4): p. 989-1002.

93. Zariri, A., et al., Meningococcal outer membrane vesicle composition-dependent activation of the innate immune response. Infection and Immunity, 2016.

94. Turner, L., et al., Increased Outer Membrane Vesicle Formation in a Helicobacter pylori tolB Mutant. Helicobacter, 2015. 20(4): p. 269-283.

95. Xiang, S.D., et al., Pathogen recognition and development of particulate vaccines: does size matter? Methods, 2006. 40(1): p. 1-9.

96. Klimentova, J. and J. Stulik, Methods of isolation and purification of outer membrane vesicles from gram-negative bacteria. Microbiol Res, 2015. 170: p. 1-9.

97. Steen, H.B., Flow cytometer for measurement of the light scattering of viral and other submicroscopic particles. Cytometry A, 2004. 57(2): p. 94-9.

98. van der Vlist, E.J., et al., Fluorescent labeling of nano-sized vesicles released by cells and subsequent quantitative and qualitative analysis by high-resolution flow cytometry. Nat Protoc, 2012. 7(7): p. 1311-26.

99. Malloy, A. and B. Carr, NanoParticle Tracking Analysis - The Halo ${ }^{\mathrm{TM}}$ System. Particle \& Particle Systems Characterization, 2006. 23(2): p. 197-204.

100. Vogel, R., et al., Quantitative sizing of nano/microparticles with a tunable elastomeric pore sensor. Anal Chem, 2011. 83(9): p. 3499-506.

101. Gao, W., et al., Modulating antibacterial immunity via bacterial membrane-coated nanoparticles. Nano Lett, 2015. 15(2): p. 1403-9.

102. Kumar, S., et al., Shape and size-dependent immune response to antigen-carrying nanoparticles. Journal of Controlled Release, 2015. 220, Part A: p. 141-148.

103. Manolova, V., et al., Nanoparticles target distinct dendritic cell populations according to their size. Eur J Immunol, 2008. 38(5): p. 1404-13. 


\section{Chapter 3}

\section{High throughput nanoparticle tracking analysis for monitoring outer membrane vesicle production}

Published as:

Gerritzen, M.J.H., D.E. Martens, R.H. Wijffels and M. Stork (2017). "High throughput nanoparticle tracking analysis for monitoring outer membrane vesicle production." Lournal of Extracellular Vesicles, 6:1, 1333883 


\begin{abstract}
Outer membrane vesicles (OMVs) are spherical membrane nanoparticles released by Gram-negative bacteria. OMVs can been quantified in complex matrices by nanoparticle tracking analysis (NTA). NTA can be performed in static mode or with continuous sample flow that results in analysis of more particles in a smaller time-frame. Flow measurements must be performed manually despite the availability of a sample changer on the NanoSight system. Here we present a method for automated measurements of OMVs in flow mode. OMV quantification in flow mode showed a mean particle concentration (coefficient of variation (CV) of $6 \%$ CV static measurements of $14 \%$ ). Sizing of OMVs was expected to be less favorable in flow mode due to the increased movement of the particles. However, we observed a CV of $3 \%$ in flow mode and a CV of $8 \%$ in static measurements. Flow rates of up to $5 \mu \mathrm{L} / \mathrm{min}$ displayed correct size and particle measurements, however, particle concentration was slightly lower than in static measurements. The automated method was used to assess OMV release of batch cultures of Neisseria meningitidis. The bacteria released more OMVs in stationary growth phase, while the size of the vesicles remained constant throughout the culture. Taken together, this study shows that automated measurements in flow mode can be established with advanced scripting to reduce the workload for the user.
\end{abstract}




\section{Introduction}

Bacterial derived nanoparticles, known as outer membrane vesicles (OMVs), have gained more and more attention in the development of bacterial vaccines [1, 2]. These OMVs are 20-200 nm spherical particles that resemble the outside of the bacterium [3]. OMVs are complex nanoparticles consisting of proteins, lipopolysaccharides, phospholipids and DNA that are involved in cellular communication, toxin delivery, surface modifications, removal of undesired components, and polysaccharide degradation [4-7].

The quantification of OMVs has been challenging since indirect methods had to be used for detection of the particles. Often, the total protein concentration of the OMV sample was used as a measure of the number of vesicles [8-12]. Another indirect method was based on the integration of a hydrophobic dye in vesicle membrane, resulting in a measurable change in absorbance [13]. These indirect methods can be largely influenced by impurities and are thus not suited to measure unpurified and intermediate samples.

Direct measurement of OMVs by nanoparticle tracking analysis (NTA) can be used for the quantification and sizing of OMVs [14]. Besides measurement of the number and size of the particles, the refractive index of individual particles can be calculated [15]. NTA can be performed in static mode by measuring the particles trapped in the measurement chamber or in flow mode by slowly flowing the nanoparticle sample through this measurement chamber. Static measurements allow the analysis of single nanoparticles in time, for example the study of nanoparticle aggregation by elevated temperatures [16]. Flow mode NTA measurements for nanoparticle quantification are advantageous over static measurements since more particles can be measured in a smaller time-frame resulting in less variance in particle quantitation [17]. NTA can be used to measure specifically fluorescent labelled particles [18]. In this fluorescence measurement, continuous measurement is advantageous since the sample flow prevents photobleaching of the fluorescent label [19].

Despite the availability of both a syringe pump and a sample changer on the NanoSight NS500, there is no option for automated measurements in flow mode available. As a result, the analysis of samples under flow mode require more time than automated static measurements. In this work we describe a method that combines the 
measurement of samples in flow mode with the sample changer to allow high throughput measurement under flow. For this, we have compared the accuracy of NTA measurements of OMVs in static measurements and in flow mode. The influence of the flow rate on the NTA analysis has been assessed. Lastly, we applied the automated measurement method to quickly assess a large set of samples to research the OMV formation in batch cultivations.

\section{Material and methods}

\section{Preparation of outer membrane vesicle stock}

A highly purified Neisseria meningitidis spontaneous released OMV (sOMV) investigational vaccine was prepared from a batch culture of Neisseria meningitidis. From this culture broth, the vesicles were separated from the bacteria by tangential flow filtration using a $0.2 \mu \mathrm{m}$ cutoff mPES hollow fiber module (Spectrum Labs, The Netherlands). Vesicles were concentrated by tangential flow filtration using a $100 \mathrm{kDa}$ cutoff mPES hollow fiber module (Spectrum Labs, The Netherlands), and the sOMVs were subsequently purified by preparative size exclusion chromatography using Sepharose ${ }^{\mathrm{TM}} 6$ Fast Flow resin (GE Healthcare Life Sciences, USA). Lastly, a dead-end sterile filtration step was performed to ensure sterility of the sOMV product before it was stored at $4^{\circ} \mathrm{C}$. To assess the total protein content of the OMV stock, a Lowry protein assay with Peterson's modification was used according to the manufacturer's protocol (Sigma-Aldrich, The Netherlands). The sOMVs were diluted in freshly tapped MilliQ water up to a concentration of approximately $8.5 \times 10^{8}$ particles $/ \mathrm{mL}$. This concentration was chosen so that the NTA measurements yield 40 to 50 particles per frame to ensure measurements are in the linear range of NTA measurements [20].

\section{Nanoparticle tracking analysis}

A NanoSight NS500 with a $488 \mathrm{~nm}$ laser module and sCMOS camera module was used for all NTA measurements (Malvern Instruments, UK). Additionally, a Gilson 223 sample changer and a Harvard Apparatus syringe pump (Catalog No. 98-4730) were connected to the NS500 by a 4-way connector as described in the results section. The syringe pump was equipped with a $500 \mu \mathrm{L}$ glass syringe (Hamilton Model $1750 \mathrm{RN}$ ). Static measurements were obtained by capturing 10 measurements of 60 -seconds of a sample loaded in the measurement chamber. Measurements in flow mode were performed with a flow rate of $25(\sim 2.6 \mu \mathrm{L} / \mathrm{min})$, yielding a y-drift of 4.0 pixels per frame. Like the static 
measurements, these flow measurements consisted of 10 measurements of 60-second, but with an additional 30-second delay between measurements. The method for automated measurements in flow mode are described in the results section. The script file used for these automated measurements can be found in the Supplemental File 1. All measurements were performed with temperature controlled at $25^{\circ} \mathrm{C}$, and the captured data of both static and flow measurements was analyzed using NTA 3.2 software build 3.2.16. The capture settings and the analysis settings of the NTA software are shown in Table 1. The machine was calibrated by the NanoSight NTA concentration Measurement upgrade. The NS500 is cleaned with Decon 90 monthly, according to the recommendations described in the manual. Before all sample measurements, we confirmed that the MilliQ diluent contained less than 1.0 particle per frame by measuring the MilliQ diluent for 60 seconds in static mode.

Table 1. NTA software settings

\begin{tabular}{ll}
\hline \multicolumn{2}{c}{ Capture settings } \\
\hline CAMERASHUTTER & 1206 \\
CAMERAGAIN & 366 \\
CAMERALEVEL & 15 \\
CAMERAHILIM & 3294 \\
CAMERALOLIM & 0 \\
STAGE & -20376 \\
FOCUS & 27 \\
\multicolumn{2}{c}{ Analysis settings } \\
\hline DETECTTHRESHOLD & 3 \\
AUTOBLUR & ON \\
AUTOMINTRACKLENGHT & ON \\
\hline
\end{tabular}

\section{Statistics}

Statistics of the static and flow NTA measurements were performed in RStudio [21], version 0.99.903. Significance of the static and flow NTA measurements was calculated by an exact, unpaired Mann-Whitney $U$ test by using the exactRankTests package version 0.8-28 [22]. 


\section{Dynamic light scattering}

DLS measurements were performed by using a Zatasizer Nano-ZS (Malvern Instruments, UK). Measurements were made in disposable polystyrene semi-micro cuvettes (Greiner bio-one, 613101) using the Zetasizer 7.11 software. A standard operating procedure (SOP) was used for the measurements with sample set as protein with a refractive index of 1.450 and an absorption of 0.001 . Water was used as dispersant with a viscosity of $0.8872 \mathrm{cP}$ and refractive index of 1.330 . Three measurements were performed using a measurement angle of $173^{\circ}$ (backscatter), auto measurement duration and "seek for optimal position" as positioning setting. Data processing was performed with the general purpose (normal resolution) analysis model.

\section{Bioreactor cultivations}

Batch cultivation for OMV stock production was performed in a 3L working volume dished bottom bioreactors with a H/D ratio of 1.0 based on working volume (Applikon Biotechnology, The Netherlands). Cultures were controlled at $35 \pm 0.5^{\circ} \mathrm{C}, \mathrm{pH} 7.2 \pm 0.05$ with $1 \mathrm{M} \mathrm{HCl}$ and $1 \mathrm{M} \mathrm{NaOH}$, and a dissolved oxygen tension of $30 \%$ using a Tryton ${ }^{\mathrm{i}}$ controller (Pierre Guerin, France). Dissolved oxygen tension was controlled by the agitation rate (300 - 1000 RPM) and the concentration of oxygen in the headspace gas flow of $1 \mathrm{NL} / \mathrm{min}$. Culture samples were sterile filtered with a disposable syringe filter with $0.22 \mu \mathrm{m}$ cutoff and stored at $4^{\circ} \mathrm{C}$. 


\section{Results}

\section{Static vs. flow measurements}

NTA can be performed by static measurements or by measurements in flow mode.

Sample flow allows more particles to be analyzed in a single capture, but the software has to correct for the particle drift in the measurement. To assess the most accurate method for OMV enumeration and OMV sizing, we compared static and continuous flow NTA measurements. From a N. meningitidis OMV stock, 100 measurements were obtained in both static and in flow-mode, by manually capturing 10 videos of 60 seconds of 10 replicate samples.

OMV quantification of the 100 static measurements showed a mean particle concentration of $9.07 * 10^{8}$ particles/mL with a coefficient of variation of $14 \%$ (Figure 1 ). Flow measurements showed a mean concentration of $8.63^{*} 10^{8}$ particles $/ \mathrm{mL}$ with a coefficient of variation (CV) of 6\%. Sizing of the particles showed a mean size of $77.6 \mathrm{~nm}$ $(\mathrm{CV}=8 \%)$ and $78.2 \mathrm{~nm}(\mathrm{CV}=3 \%)$ for resp. static and continuous flow measurements. The lower measurement variation in for both particle size and particle number in the flow measurements can be explained by the increased number of particles analyzed due
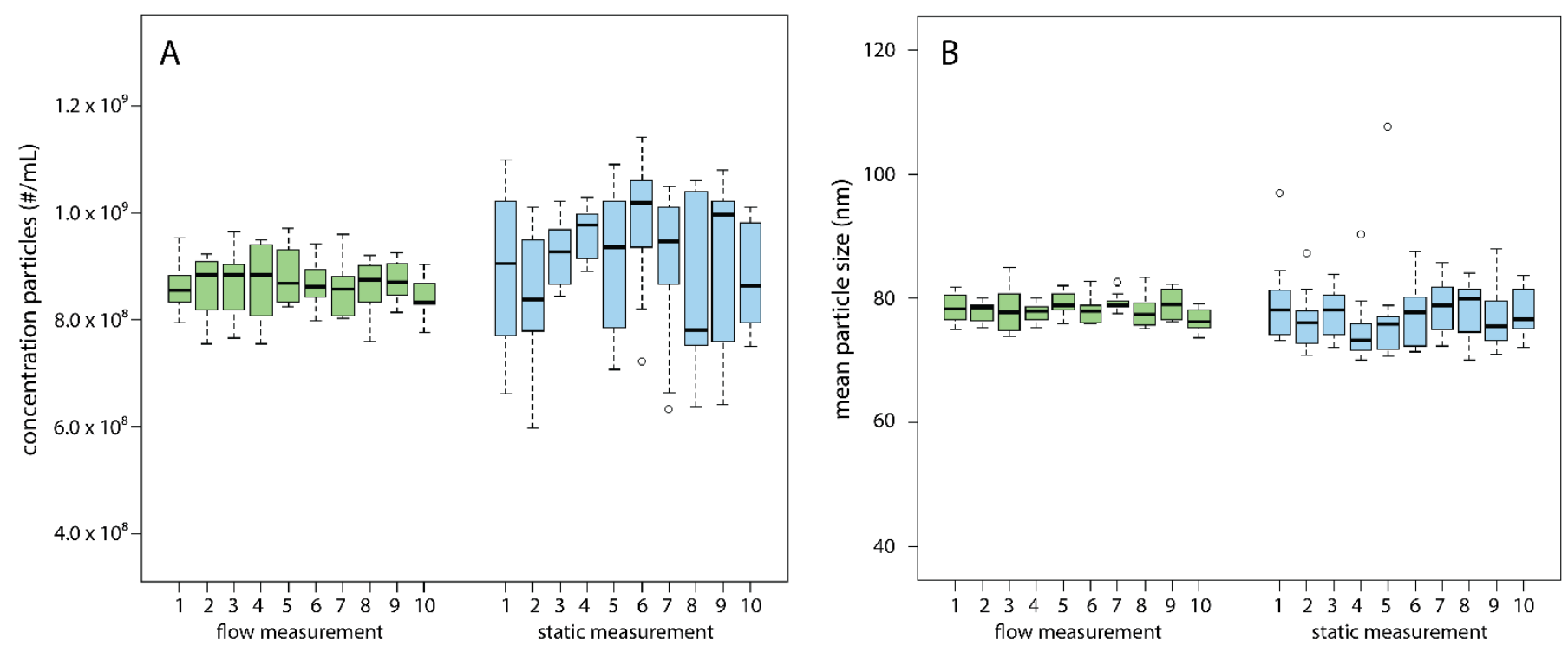

Figure 1. Static (blue) and flow (green) measurements of outer membrane vesicles. Boxplot A shows the result of NTA particle concentration measurement and plot B of NTA particle sizing. Boxes represent 10 measurements of 60 seconds. Outliers represent measurements 1.5 times the interquartile range below the lower quartile or above the upper quartile. 
to the increased observation volume. The reduced variance in the size measurement under flow shows that the software is capable of correcting for the particle drift. Based on the lower variation in the continuous flow measurement, the number of captures per sample measurement could be reduced and we used this method of NTA for the automated quantification of OMVs.

\section{Automated flow measurements}

To automate the sample measurement in flow mode, we connected both the sample changer and the syringe pump to a NanoSight NS500 (Figure 2). The connection was made with a 4-way valve allowing the sample to be loaded in to the syringe pump before the sample was measured. The flow path through the 4 -way valve was controlled by the two pumps on the NanoSight and the syringe-pump. All pumps block their flow path when switched off so that the flow path can be directed by rational programming of the pumps. To realize the flow from the autosampler to the syringe pump, which crosses two pumps, the syringe pump was calibrated with the integrated pump of the NanoSight. The approach of this programming is described in this section and the developed script for automated continuous flow measurements can be found in Supplemental File 1.

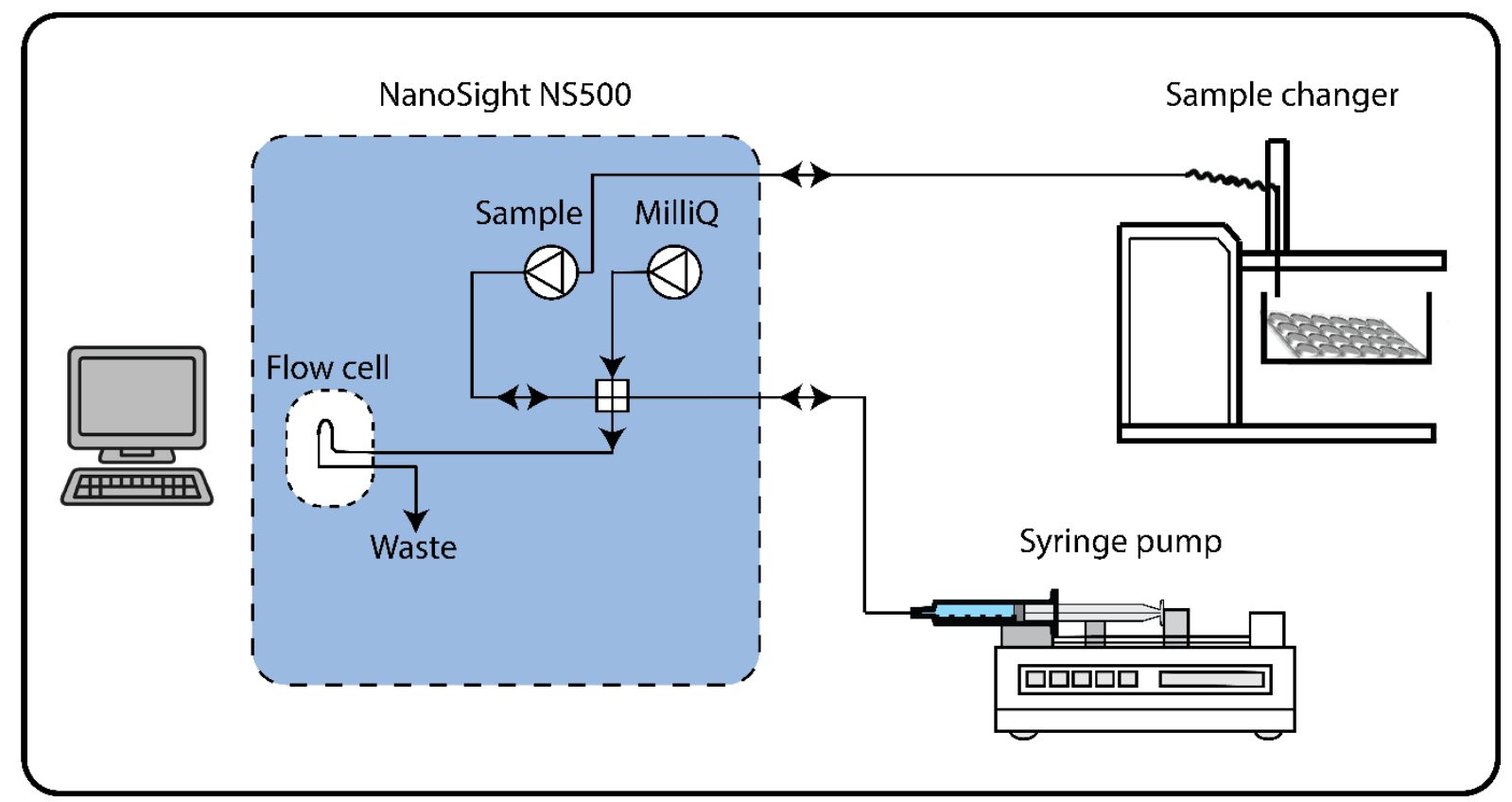

Figure 2. The schematic setup of the NanoSight setup for automated flow measurements. Both the sample changer and the syringe pump are connected to the NanoSight by a 4-way valve. 
Automated continuous flow measurements start with an initialization phase, followed by the measurement phase and end by a finalization phase (Table 2). In the initialization phase (INIT) the syringe pump, sample changer and the measurement cell are flushed with MilliQ. The MilliQ is measured to confirm the absence of particles in the MilliQ supply. During the measurement phase (MEAS) the number of measurements can be set by a loop from MEAS1 to MEAS7. Within this loop the sample is transferred from the sample changer to the syringe pump, before the sample is loaded in to the measurement chamber. A second loop is programmed in MEAS3 which determines the number of captures per sample. After each measurement, sample changer, the syringe, and the measurement chamber are washed with MilliQ water before a new sample is measured. In the finalization phase (FINA) the captures are processed and the result can be exported.

Table 2. Schematic overview of the script for automated measurement in flow mode

\begin{tabular}{ll} 
Step & Action \\
\hline INIT1 & Flush syringe with MilliQ \\
INIT2 & Flush sample changer with MilliQ \\
INIT3 & Measure particle background of MilliQ stock \\
INIT4 & $\begin{array}{l}\text { Set the number and positions of the samples } \\
\text { in the sample changer }\end{array}$ \\
MEAS1 & Load the syringe with sample \\
MEAS2 & Load measurement chamber with sample \\
MEAS3 & Measurement of the sample \\
MEAS4 & Emptying the syringe \\
MEAS5 & Washing the syringe \\
MEAS6 & Wash sample changer \\
MEAS7 & Processing of measurement \\
FINA2 & Exporting results \\
FINA3 & Shutdown
\end{tabular}

For a measurement in flow mode, the sample was loaded from the sample changer to the syringe in the syringe pump. We ensured the purity of the sample by setting the 
sample pump at a slightly higher rate than the syringe pump. As a result, a small fraction of the sample was directly flushed through the measurement chamber to the waste. Similarly, this methodology was used to flush the syringe pump and the measurement chamber with MilliQ.

Washing of the syringe pump was required to prevent cross contamination between samples. A single flush cycle consists of emptying the syringe and loading the syringe with MilliQ. To complete the wash cycle, the syringe was emptied. Because of the inevitable holdup volume of the tubing and in the connection with the syringe, sample cross contamination could be detected. After 1 flush cycle, sample carryover of approximately $5 \%$ was observed. The cross contamination for up to 5 wash cycli was tested (Figure 3). In the first two flushes particles derived from the measured OMV stock could be detected. The particle concentration after 3 flushes showed a similar background particle concentration as the MilliQ water. An excess of 5 flush cycles of the syringe was programmed in the script for automated continuous flow measurements to minimize the possibility of cross contamination between samples.

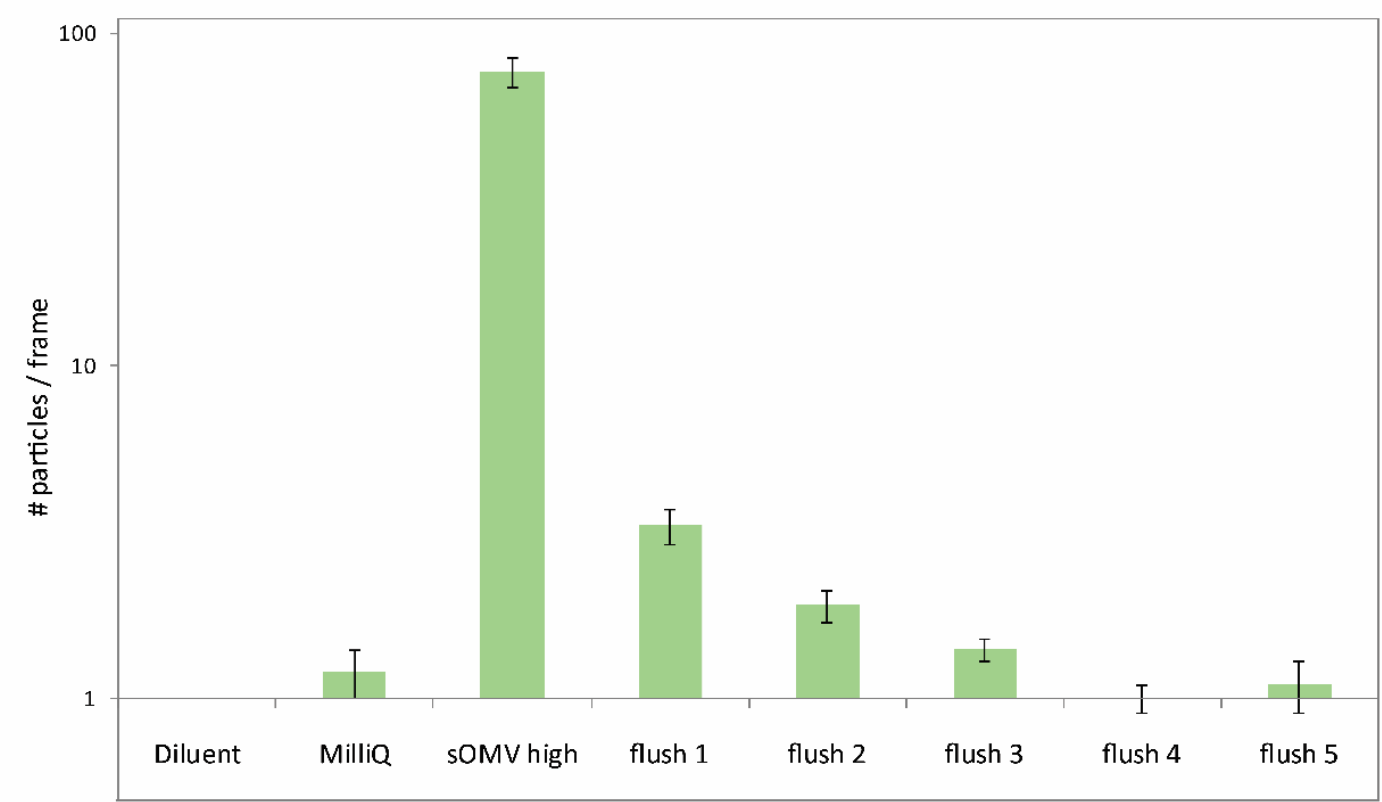

Figure 3. Automated cleaning of the syringe pump. NTA measurements were made from the diluent MilliQ water and MilliQ water loaded in the syringe to assess background particles. A OMV stock yielding 80 particles per frame was prepared and measured by recording a single 60 second capture. Next the syringe was emptied and filled with MilliQ and measured again (Flush 1). Flushes were repeated to assess the carry-over of particles. 


\section{Influence of the sample flow rate on NTA}

NTA measurements in flow mode showed a significant lower $(\mathrm{p}=0.0003)$ particle concentration of 5\% compared to static measurements (Figure 1). It is unknown which value resembles the exact value of the OMV stock solution the closest. To assess the possible influence of the flow rate on the particle quantification, we have measured the OMV stock at different flow rates.

The OMV stock was measured in static mode and at flow rates up to 200 units of the NTA software. This maximum flow rate corresponds, in combination with the $500 \mu \mathrm{L}$ syringe, to a liquid flow of $21 \mu \mathrm{L} / \mathrm{min}$ (Figure 4, A). NTA analysis showed that the detected particle drift linearly correlates to the flow rate up to a flow of $15 \mu \mathrm{L} / \mathrm{min}$ (Figure 4, B). The tracking of particles and the calculated particle concentration shows to be constant at flow rates up to $5 \mu \mathrm{L} / \mathrm{min}$ (Figure $4, \mathrm{C}-\mathrm{D}$ ). Flow rates of $10 \mu \mathrm{L} / \mathrm{min}$ and higher showed to result in unrealistic low particle concentrations. The mean size of the OMVs analyzed in flow measurements up to $5 \mu \mathrm{L} / \mathrm{min}$ was comparable to measurements by DLS. Similar to concentration measurements, size measurements were not accurate at flow measurements of $10 \mu \mathrm{L} / \mathrm{min}$ and higher (Figure $4, \mathrm{E}-\mathrm{F}$ ). The flow rate of $2.6 \mu \mathrm{L} / \mathrm{min}$ showed to be in the proper range for both size and concentration measurements and is therefore used in the script for automated measurements in flow mode. 

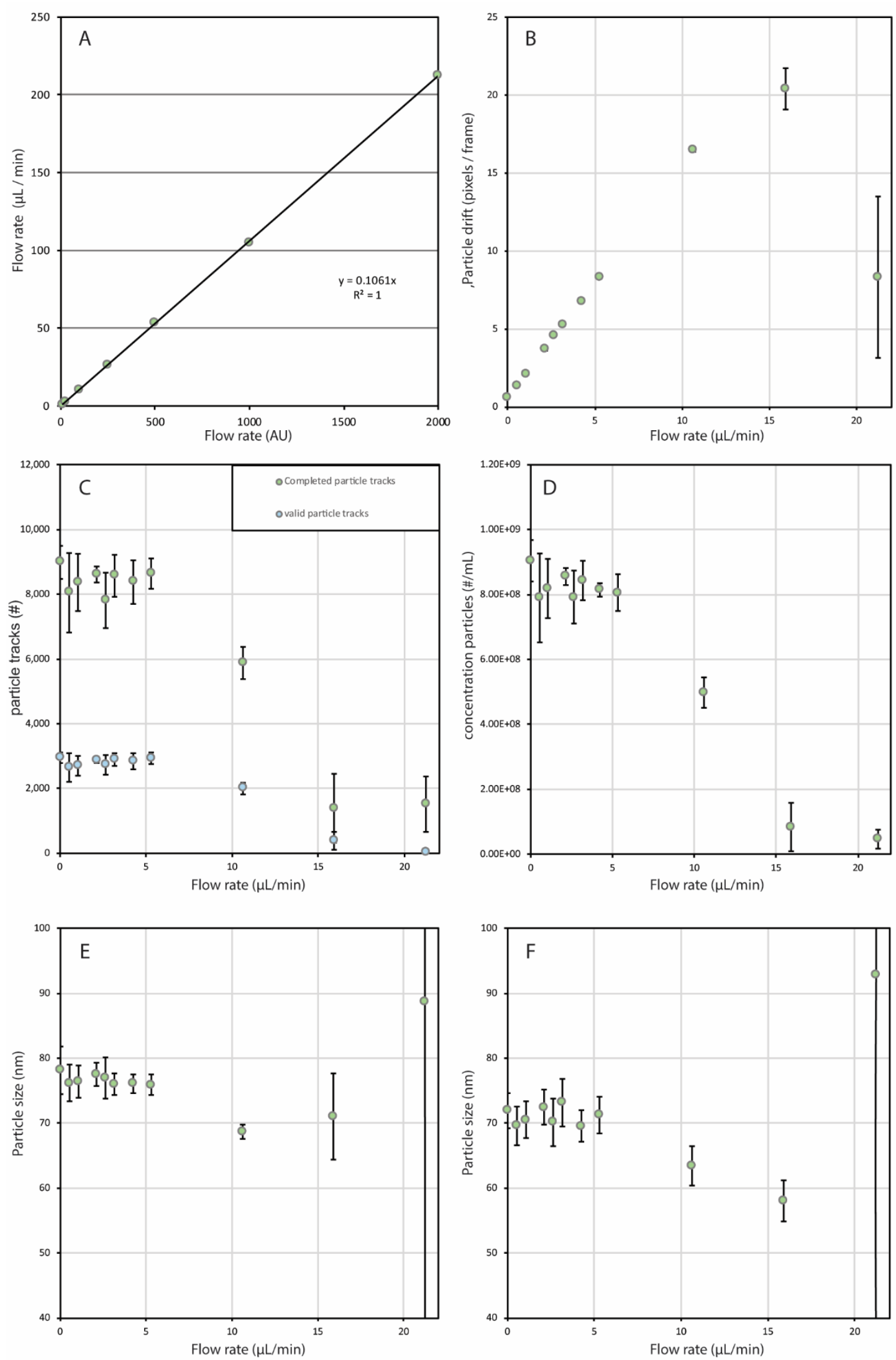

Figure 4. The influence of sample flow rate on NTA. The artificial flow rate of the NTA software has been calibrated using a $500 \mu \mathrm{L}$ syringe (A). OMV stock was measured at different flow rates. The observed particle drift (B), total and valid particle tracks (C), concentration (D), mean size (E) and mode size (F) are plotted against the sample flow rate 


\section{OMV release by Neisseria meningitidis}

The script for automated measurements in flow mode was used to assess the OMV release during bacterial growth. Three $N$. meningitidis cultures were grown in benchtop bioreactors and supernatant samples were analyzed by NTA (Figure 5). Between $2 \mathrm{~h}$ and $8 \mathrm{~h}$ the growth is exponential and the observed release of vesicles is low. The OMV release shows an increase in the stationary phase, $10-18 \mathrm{~h}$. Interestingly the size of the vesicles remains similar during the whole duration of the cultivation (Figure 5).

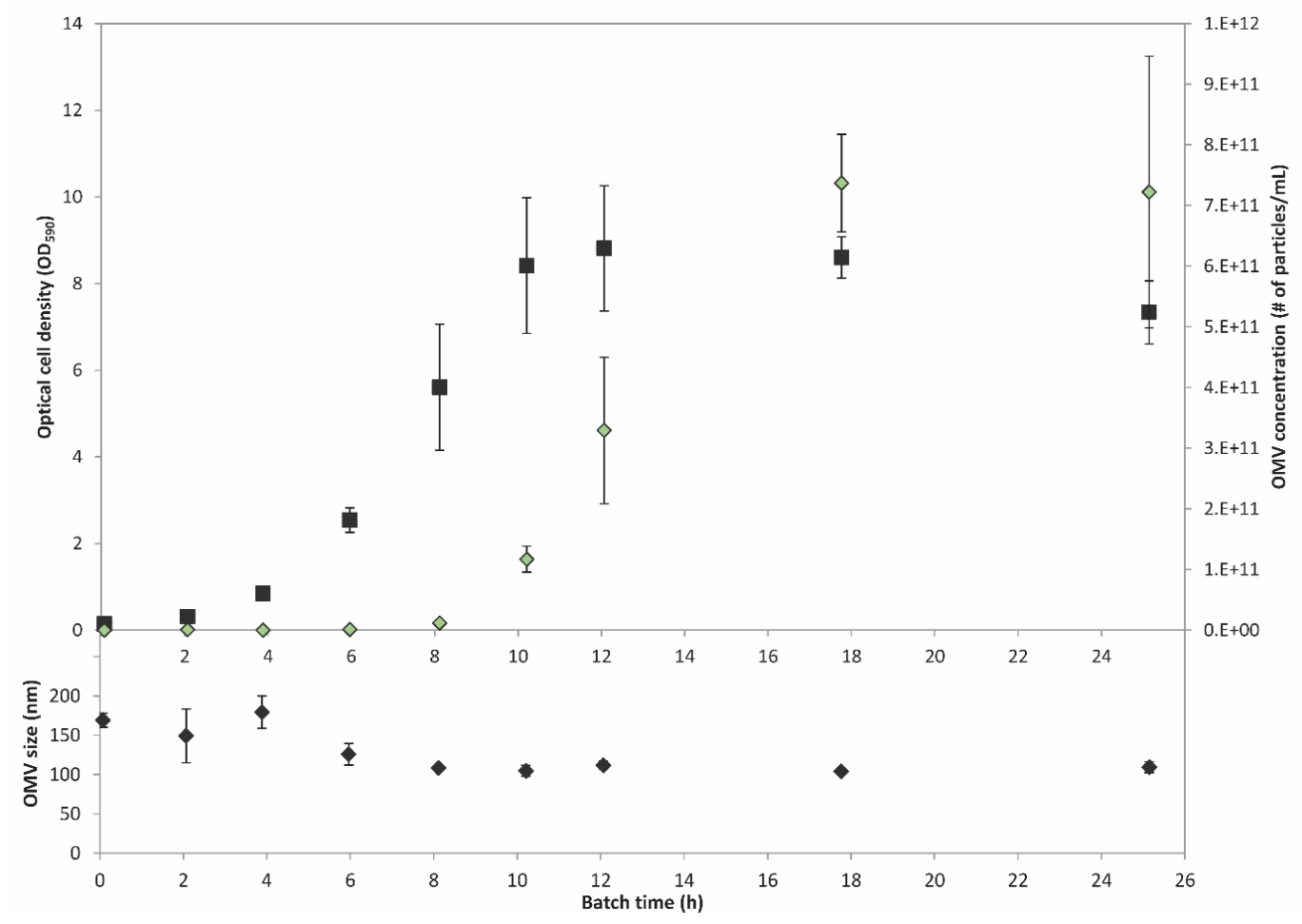

Figure 5. Growth of Neisseria meningitidis in a benchtop batch cultivation. Upper panel shows the growth pattern of the bacteria (black) and shows that OMVs (green) are increasingly produced after the exponential growth phase. The lower panel shows the size of OMVs. The size of OMVs released in the exponential growth phase is similar to the size of OMVs produced in the stationary phase. The increased size of OMVs in the first 6 hours of the cultivations deviates, possibly caused by the increased measurement error due to concentrations in the range of the lower limit of detection.

\section{Discussion}

Continuous flow NTA measurements were automated using the sample changer in combination with the scripting possibilities of the NTA software. NTA measurements of an OMV stock showed that continuous flow measurements are advantageous over static measurements in quantifying and sizing of OMVs. The automated method severely reduced the workload per measurement. Steppert et al. report that static NTA measurements on a NanoSight LM10 requires 30 to 45 min time per sample [23], which 
corresponds to our experience with static NTA on the NS500. A small fraction of this time is required for sample preparation, which makes it advantageous to automate the measurement. Automated NTA can also be used for samples of unknown concentration whereas serial dilutions can be processed by the automated method described here. Cross-contamination of too-concentrated samples is prevented by the number of syringe wash steps in the automated measurement. Overall the workload for analysing 24 samples by the automated method is roughly 2 hours. Depending on the chosen capture settings, the required measurement time of the equipment is around 30 minutes per sample. The automated method is not limited to OMV samples and theoretically the method can be applied to all samples analysed by flow-mode NTA.

NTA at different flow rates showed that flow measurements applying flows of 10 $\mu \mathrm{L} / \mathrm{min}$ or higher altered the measured particle concentration and the measured size for the N. meningitidis OMV stock. This effect has also been shown by NanoSight for the analysis of $100 \mathrm{~nm}$ polystyrene latex beads [24]. In their measurement a higher flow rate of 20 to $50 \mathrm{AU}$ (in combination with a $1 \mathrm{~mL}$ syringe, corresponding to approximately 4 to $11 \mu \mathrm{L} / \mathrm{min}$ ) showed to be ideal [17, 24]. The difference between these two measurements are the particles used and their refractive index, although the refractive index is not expected to influence the concentration measurement. Recent studies suggest that flow measurements alter the observed concentration of various extracellular vesicles already at low flow rates [25-27]. Here we also observed a minor difference between static and flow measurements. This study showed that flow rates below $10 \mu \mathrm{L} / \mathrm{min}$ are suited for OMV quantification and sizing.

The batch-wise cultivation of $N$. meningitidis showed an increased OMV release in the stationary growth phase. This pattern of release was similar to the previously described OMV release that was analysed by the hydrophobic dye Synaptored C2 [28]. By using NTA, we showed that the size of OMVs remains constant during the cultivation, although in the first hours of the cultivation the size shows to be increased. This is probably caused by particle concentration near the limit of detection although it could be possible that the observed switch in the culture could affect the size of the produced OMVs. Gene regulation may be of possible influence to OMV size since it has been previously shown that OMV size differs between mutants with deletion of genes possibly involved in the OMV biogenesis [29]. 
Alternative methods for combined OMV quantification and sizing are high-resolution flow cytometry (hFC) [30] and tunable resistive pulse sensing (TRPS) [31, 32]. These two techniques have recently been compared to static NTA for the analysis of extracellular vesicles by two groups $[33,34]$. Both groups conclude that $h F C$, TRPS and NTA are suited to quantifying and sizing extracellular vesicles. The reduction in workload required for NTA measurements can be an important consideration in the choice of analysis techniques, whereas automated sample measurement is not available for TRPS.

The automated method of NTA reduced the workload, but several practical implications should be noted. The automated method can be used for large numbers of samples, but when samples were applied in open (Eppendorf) tubes, we observed increased concentrations of particles. It appeared that the increased concentration was caused by evaporation before the sample was loaded in the measurement chamber. The use of sample vials with a septum solved this issue. With this approach, diluted samples can be in the sample changer for extended periods of time prior to measurement. OMVs are highly stable, however less stable samples could benefit by automated dilutions directly prior to measurement. The current hardware could be compatible with automated sample dilutions and adding this feature will be an improvement to the NanoSight system. Furthermore, the extended time-span of using the equipment may cause gas bubbles in the measurement chamber. These bubbles can interfere with the measurement by expelling sample from the observation volume or by disturbing the liquid flow in the measurement chamber. Gas bubble formation was avoided by degassing the MilliQ water bottle by vacuum degasification. A last practical adjustment to the equipment was made by replacing the plastic syringe for a glass version to prevent particles from sticking to the syringe and to reduce the number of flushes required between the measurement of samples.

In conclusion, the automation of continuous flow measurements can be applied with the described script on all NanoSight equipment when connected to both a sample changer and a syringe pump. This method can improve both the quality of the measurement while the workload is reduced simultaneously. Furthermore, the script secures the measurement settings, which further standardizes the use of NTA. 


\title{
Acknowledgements
}

\author{
We thank Patrick Hole for his constructive feedback on the particle concentration \\ measurements at different flow rates. This work was supported by the Dutch Ministry \\ of Health, Welfare and Sport.
}

\section{References}

1. Acevedo, R., et al., Bacterial outer membrane vesicles and vaccine applications. Front Immunol, 2014. 5: p. 121.

2. van der Pol, L., M. Stork, and P. van der Ley, Outer membrane vesicles as platform vaccine technology. Biotechnol J, 2015. 10(11): p. 1689-706.

3. Kulp, A. and M.J. Kuehn, Biological functions and biogenesis of secreted bacterial outer membrane vesicles. Annu Rev Microbiol, 2010. 64: p. 163-84.

4. Haurat, M.F., W. Elhenawy, and M.F. Feldman, Prokaryotic membrane vesicles: new insights on biogenesis and biological roles. Biol Chem, 2015. 396(2): p. 95-109.

5. Schwechheimer, C. and M.J. Kuehn, Outer-membrane vesicles from Gram-negative bacteria: biogenesis and functions. Nat Rev Microbiol, 2015. 13(10): p. 605-19.

6. Mashburn, L.M. and M. Whiteley, Membrane vesicles traffic signals and facilitate group activities in a prokaryote. Nature, 2005. 437(7057): p. 422-5.

7. McBroom, A.J. and M.J. Kuehn, Release of outer membrane vesicles by Gram-negative bacteria is a novel envelope stress response. Mol Microbiol, 2007. 63(2): p. 545-58.

8. van de Waterbeemd, B., et al., Improved production process for native outer membrane vesicle vaccine against Neisseria meningitidis. PLoS One, 2013. 8(5): p. e65157.

9. Serruto, D., et al., The new multicomponent vaccine against meningococcal serogroup B, 4CMenB: immunological, functional and structural characterization of the antigens. Vaccine, 2012. 30 Suppl 2: p. B87-97.

10. Salverda, M.L.M., et al., Surface display of a borrelial lipoprotein on meningococcal outer membrane vesicles. in prep, 2015.

11. Fantappie, L., et al., Antibody-mediated immunity induced by engineered Escherichia coli OMVs carrying heterologous antigens in their lumen. J Extracell Vesicles, 2014. 3.

12. Daleke-Schermerhorn, M.H., et al., Decoration of outer membrane vesicles with multiple antigens by using an autotransporter approach. Appl Environ Microbiol, 2014. 80(18): p. 5854-65.

13. McBroom, A.J., et al., Outer membrane vesicle production by Escherichia coli is independent of membrane instability. J Bacteriol, 2006. 188(15): p. 5385-92.

14. Malloy, A. and B. Carr, NanoParticle Tracking Analysis - The Halo ${ }^{\text {TM }}$ System. Particle \& Particle Systems Characterization, 2006. 23(2): p. 197-204.

15. Gardiner, C., et al., Measurement of refractive index by nanoparticle tracking analysis reveals heterogeneity in extracellular vesicles. Journal of Extracellular Vesicles; Vol 3 (2014) incl supplements, 2014.

16. Filipe, V., A. Hawe, and W. Jiskoot, Critical Evaluation of Nanoparticle Tracking Analysis (NTA) by NanoSight for the Measurement of Nanoparticles and Protein Aggregates. Pharmaceutical Research, 2010. 27(5): p. 796-810.

17. MalvernInstruments, NanoSight NTA Concentration Measurement Upgrade, in TN150515. 2015.

18. Malloy, A., Count, size and visualize nanoparticles. Materials Today, 2011. 14(4): p. 170-173.

19. Gardiner, C., et al., Extracellular vesicle sizing and enumeration by nanoparticle tracking analysis. Journal of Extracellular Vesicles; Vol 2 (2013) incl Supplements, 2013.

20. Dragovic, R.A., et al., Sizing and phenotyping of cellular vesicles using Nanoparticle Tracking Analysis. Nanomedicine, 2011. 7(6): p. 780-8.

21. RStudio, T. RStudio: integrated development for R. RStudio, Inc., Boston, MA 2015; Available from: http://www.rstudio.com.

22. T. Hothorn and K. Hornik. exactRankTests: exact distributions for rank and permutation tests. $R$ package version 0.8-28. . 2015; Available from: http://CRAN.R-project.org/package=exactRankTests.

23. Steppert, P., et al., Quantification and characterization of virus-like particles by size-exclusion chromatography and nanoparticle tracking analysis. Journal of Chromatography A, 2017. 1487: p. 8999. 
24. MalvernInstruments, Enhancing NTA accuracy and repeatability with flow mode measurements. TN160808, 2016.

25. Xu, T., et al., Efficient Genome Editing in Clostridium cellulolyticum via CRISPR-Cas9 Nickase. Appl Environ Microbiol, 2015. 81(13): p. 4423-31.

26. Krueger, A.B., P. Carnell, and J.F. Carpenter, Characterization of Factors Affecting Nanoparticle Tracking Analysis Results With Synthetic and Protein Nanoparticles. Journal of Pharmaceutical Sciences, 2016. 105(4): p. 1434-1443.

27. Tong, M., et al., Flow speed alters the apparent size and concentration of particles measured using NanoSight nanoparticle tracking analysis. Placenta, 2016. 38: p. 29-32.

28. van de Waterbeemd, B., et al., Cysteine depletion causes oxidative stress and triggers outer membrane vesicle release by Neisseria meningitidis; implications for vaccine development. PLoS One, 2013. 8(1): p. e54314.

29. Deatherage, B.L., et al., Biogenesis of bacterial membrane vesicles. Mol Microbiol, 2009. 72(6): p. 1395407.

30. van der Vlist, E.J., et al., Fluorescent labeling of nano-sized vesicles released by cells and subsequent quantitative and qualitative analysis by high-resolution flow cytometry. Nat. Protocols, 2012. 7(7): p. 1311-1326.

31. Vogel, R., et al., Quantitative sizing of nano/microparticles with a tunable elastomeric pore sensor. Anal Chem, 2011. 83(9): p. 3499-506.

32. Coumans, F.A.W., et al., Reproducible extracellular vesicle size and concentration determination with tunable resistive pulse sensing. Journal of Extracellular Vesicles; Vol 3 (2014) incl supplements, 2014.

33. van der Pol, E., et al., Particle size distribution of exosomes and microvesicles determined by transmission electron microscopy, flow cytometry, nanoparticle tracking analysis, and resistive pulse sensing. J Thromb Haemost, 2014. 12(7): p. 1182-92.

34. Maas, S.L., et al., Possibilities and limitations of current technologies for quantification of biological extracellular vesicles and synthetic mimics. J Control Release, 2015. 200: p. 87-96.

\section{Supplemental File 1}

The "script automated NTA in flow mode.txt" is available as supporting information from:

https://www.tandfonline.com/doi/suppl/10.1080/20013078.2017.1333883/suppl fil e/zjev a 1333883 sm9625.txt 


\section{Chapter 4}

\section{Sulfate depletion triggers overproduction of phospholipids and the release of outer membrane vesicles by \\ Neisseria meningitidis}

Accepted for publication:

Gerritzen, M.J.H., D.E. Martens, J.P. Uittenbogaard, R.H. Wijffels and M. Stork. "Sulfate depletion triggers overproduction of phospholipids and the release of outer membrane vesicles by Neisseria meningitidis."

$\underline{\text { Scientific Reports (2019) }}$ 


\begin{abstract}
Outer membrane vesicles (OMVs) produced by bacteria are interesting vaccine candidates. OMVs are nanoparticles that contain many immunogenic components, are self-adjuvating, and non-replicative. Despite recent insights in the biogenesis of OMVs, there is no consensus on a conserved mechanism of OMV release and the OMV yield from bacterial cultures remains low. For Neisseria meningitidis, a Gram-negative human pathogen causing meningitis and sepsis, a feasible OMV production method based on triggering OMV release by cysteine depletion has been described. In this study, we investigated the mechanism behind this external trigger for OMV release to improve the production process. Since enhanced OMV release upon cysteine depletion was associated with oxidative stress and redox responses, we investigate the influence of more oxidized sulfur sources on OMV release. We show that $N$. meningitidis grows similarly on sulfate, the most oxidized sulfur source, and OMV release is triggered by sulfur depletion in general. Sulfate depletion induced increased release of OMVs over cysteine depletion. Proteomics showed that sulfur depletion resulted in oxidative stress responses and upregulated phospholipid and LPS biosynthesis. Furthermore, OMVs produced by sulfur depletion were enriched in phospholipids. Mechanistically, we hypothesize that sulfur depletion results in overproduction of phospholipids causing increased bulging of the outer membrane and subsequent OMV release.
\end{abstract}




\section{Introduction}

Outer membrane vesicles (OMVs) are spherical nanoparticles that contain the natural components of the bacterial outer membrane, including antigenic components [1]. OMVs have been effectively used as vaccines [2-6]. The OMV-based vaccines are currently not based on OMVs that are spontaneously released by the bacteria (sOMVs), but on vesicles extracted by a detergent (dOMVs). This extraction method was required to remove endotoxic lipopolysaccharides (LPS) from the OMVs, to make them suitable as vaccine. However, detergent extraction also caused a reduction in lipoproteins, reduced vesicle stability, and increased amounts of cytoplasmic components, all of which lower the vaccine quality [7]. Recently, LPS has been genetically engineered to reduce the toxicity of LPS by altering the LPS structure $[8,9]$. This approach of genetic LPS detoxification makes the detergent extraction obsolete and allows much milder detergent-free extraction methods. These milder OMV extraction methods can be used to produce detergent-free extracted OMVs (eOMVs) with improved yield, stability and immunogenicity as compared to dOMVs $[10,11]$. Alternatively, genetic detoxification of LPS also allows the use of sOMVs as vaccines.

Using sOMV over eOMVs simplifies the vesicle production process. OMV extraction requires several additional unit operations while sOMVs could be directly obtained from the culture supernatant. Another advantage of using spontaneous vesicles as vaccines is the enhanced immunogenicity of the vesicles [10]. Compared to both $\mathrm{Nm}$ eOMVs and dOMVs, the evoked immune response of sOMVs from Neisseria meningitidis $(\mathrm{Nm})$ is higher and shows a broader cross-protection against PorA types that are not included in the vesicles itself [10]. sOMV production however, is challenging since typical sOMV productivity is low. The release of OMVs has been proposed as a regulated mechanism based on the observation that OMVs differ in protein composition from the bacterial outer membrane [12-16]. Many biological functions have been ascribed to OMVs, that are advantageous for the survival chances of the bacterium by for example delivery of virulence factors or modulation of host immune response $[1,17]$. Progression in understanding OMV biogenesis has been made, however the mechanism of OMV formation remains unclear and even the question remains whether a conserved mechanism exists $[7,18]$. The biogenesis of OMVs has been categorized in three models [19]. In a first model, OMV release is based on the maintenance of lipid asymmetry in the outer membrane, that is based on increased phospholipid content in the outer 
leaflet of the outer membrane instead of LPS. A second model proposes that OMV release is induced by misfolded and unfolded proteins in the periplasm. In a third model, OMV formation is induced by alterations in the stability of the outer membrane due to LPS modifications.

Since the exact biogenesis of OMVs remains unknown, engineering approaches were applied to improve OMV productivity. One approach is based on the observation of increased vesicle release by strains with a reduced linkage of the outer membrane to the peptidoglycan [1]. It has been shown for several bacterial species that reducing the OM-peptidoglycan linkage, by knockout mutation of membrane anchoring proteins, increases the release of OMVs [10, 20-23]. In this work, we use the knockout of the membrane anchoring RmpM protein to increase the sOMV release of Nm. Additionally, we showed previously that cysteine depletion could be used as external trigger of $\mathrm{Nm}$ OMV release in a batch culture [24]. Cysteine depletion resulted in impaired sulfur supply, the onset of the stationary growth phase, oxidative stress as shown by upregulation of genes involved in oxidative stress responses, and in an increased OMV release. However, the mechanism of this increased vesicle formation remains unknown. Based on the fact that oxidative stress responses seem to be involved in OMV release, we hypothesize that the oxidation state of the sulfur source affects the release of OMVs. Sulfur is an essential component of proteins and the sulfur metabolism of $\mathrm{Nm}$ has been extensively studied [25-28]. Originally it was described that reduced sulfur (cysteine or cystine) is necessary for growth $[25,26]$, and Catlin described an absolute requirement of cysteine for growth of a few Nm strains [29]. However, growth of Neisseria on the oxidized sulfur source sulfate has been shown [27, 28], indicating that Nm metabolism is capable of sulfate reduction. The cysteine biosynthesis in Neisseria species has been recently reviewed [30]. For the assimilation of cysteine from sulfate, sulfate is converted to adenosine 5'-phosphosulfate (APS) and via phosphoadenosine-5'-phosphosulfate (PAPS) the sulfur is reduced to sulfite [31]. After further reduction of sulfite to hydrogen sulfide the sulfur is incorporated in cysteine. It has been proposed that the Nm pathway may differ slightly from the described classical cysteine assimilatory pathway, since APS could be directly reduced to sulfite without PAPS as intermediate [31]. Besides sulfate, thiosulfate can be used as well for the assimilation of sulfur source since the presence of thiosulfate reductase has been shown [28]. Growth of Nm on oxidized sulfur sources is 
thus possible and the availability of multiple sulfur-acquisition routes illustrates the importance of sulfur in the metabolism of meningococci.

This study aims to increase the understanding of OMV formation in Nm triggered by sulfur depletion by looking at the influence of different sulfur sources on OMV release upon sulfur depletion. First, we assess growth of $\mathrm{Nm}$ on sulfate as the sole sulfur source in a chemically defined medium. Next, the effect of cysteine and sulfate depletion is assessed by proteomics. Lastly, to gain further insight in the mechanism of OMV biogenesis upon sulfur-source depletion, the biochemical composition of OMVs produced in different growth phases and upon both cysteine and sulfate depletion was assessed.

\section{Materials and Methods}

\section{Shaker flask cultures}

A recombinant derivate of the Neisseria meningitidis serogroup B isolate H44/76 [48] was used in this study. This strain had a non-encapsulated phenotype due to the siaD knockout, IpxL1 deletion to attenuate LPS-toxicity, rmpM deletion to improve vesicle formation and $\operatorname{lgt} B$ was mutated to skew towards dendritic cells [10, 49]. The selected strain was a PorA lacking derivate of the H44/76 isolate. This strain was stored in a two-tiered seedlot system containing glycerol at $-135^{\circ} \mathrm{C}$. All cultivations were performed with chemically defined growth medium [50]. Growth on sulfate, sulfite and thiosulfate was performed with adapted strains since cysteine is the preferred sulfur source. Adaptation was performed by subculturing the strain in shaker flasks in medium without cysteine (Supplemental Fig. 1). Initial growth was observed, possibly caused by carryover of cysteine, followed by an adaptation phase of 26 hours. After exponential growth on sulfate was observed, the culture was subcultured on medium with sulfate as the only sulfur source and cryopreserved for further experiments. Shaker flask cultivations of $150 \mathrm{~mL}$ were performed in $500 \mathrm{~mL}$ baffled shaker flasks incubated at $200 \mathrm{RPM}$ at $35^{\circ} \mathrm{C}$. Cultures were inoculated from a shaker flask culture in the exponential growth phase. Samples with a fixed volume of $2.0 \mathrm{~mL}$ were taken for optical density measurements (590 nm), pH measurements and used for sOMV concentration measurements after sterile filtration $(0.22 \mu \mathrm{m})$ and storage at $4^{\circ} \mathrm{C}$. 


\section{Bioreactor cultures}

Batch cultivations were performed in 5 liter dished bottom Applikon bioreactors with an H/D ratio of 1.6 based on total volume. Cultivations with a 3 liter working volume

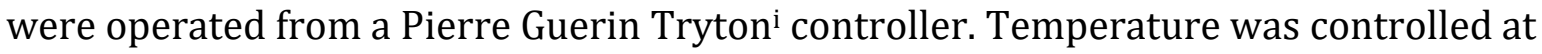
$35 \pm 0.5^{\circ} \mathrm{C}$ and $\mathrm{pH}$ was controlled at $\mathrm{pH} 7.2 \pm 0.05$ using $1 \mathrm{M} \mathrm{HCl}$ and $1 \mathrm{M} \mathrm{NaOH}$ as titrant. Dissolved oxygen tension (DOT) was controlled at 30\%. In the first phase of the cultivation, DOT is controlled by the stirrer (300 - 1000 RPM) and next the fraction of oxygen is increased in the headspace aeration $(1 \mathrm{NL} / \mathrm{min})$. Samples were taken for optical density measurements and used for nutrient and sOMV concentration measurements after sterile filtration $(0.22 \mu \mathrm{m})$ and storage at $4^{\circ} \mathrm{C}$. Offgas composition was measured by a Thermo Scientific Prima $\delta b$ process mass spectrometer.

\section{Proteomics}

Protein Digestion: bacterial suspensions were denaturated at $100^{\circ} \mathrm{C}$ for $30 \mathrm{~min}$ in a potassium phosphate buffer $(100 \mathrm{mM}, \mathrm{pH} 7.8)$ containing $0.1 \%$ Rapigest (Waters) at a protein concentration $0.2 \mathrm{mg} / \mathrm{mL}$. Proteins were digested with $0.25 \mu \mathrm{g}$ endoproteinase Lys-C (Roche) for $4 \mathrm{~h}$ at $37^{\circ} \mathrm{C}$ followed by overnight digestion with $1 \mu \mathrm{g}$ trypsin (Promega) at $37^{\circ} \mathrm{C}$.

Peptide Labeling: relative quantification of proteins in each protein sample was performed using dimethyl labeling of peptides and a common reference as internal standard as described previously [51]. Individual protein digests were incubated with native formaldehyde ( $\mathrm{CH}_{2} \mathrm{O}$; Sigma-Aldrich) and $\mathrm{NaCNBH}_{3}$ (Sigma-Aldrich) in final concentrations of $50 \mathrm{mM}$. A common reference was prepared by mixing thoroughly 20 $\mu \mathrm{g}$ of each unlabeled digest. The common reference was incubated with deuteriumlabeled formaldehyde ( $\mathrm{CD}_{2} \mathrm{O}$; Sigma-Aldrich) and $\mathrm{NaCNBH}_{3}$ in final concentrations of 50 $\mathrm{mM}$. The individual dimethylated $\left(\mathrm{C}_{1} \mathrm{H}_{3}\right)_{2}$ peptide samples were mixed with the deuterium dimethylated $\left(\mathrm{C}_{1} \mathrm{D}_{2} \mathrm{H}_{1}\right)_{2}$ common reference in equal amounts. Analytes were purified by solid phase extraction (SPE) using $1 \mathrm{~mL}$ Sep-Pack C18 cartridges (Waters) according to the manufacturer's protocol. Peptides were recovered from the SPE using $60 \%$ acetonitrile and $0.1 \%$ formic acid and dried using a vacuum centrifuge (Eppendorf). Differentially labeled peptide mixtures were dissolved in $100 \mu \mathrm{L}$ water/DMSO/formic acid (94.9/5/0.1\%) and diluted 1:20 in water/DMSO/Formic acid $(94.9 / 5 / 0.1 \%)$. 
Peptide Identification by LC-MS/MS Analysis: the peptide samples were analyzed by nanoscale reversed-phase liquid chromatography electrospray mass spectrometry using an Orbitrap Fusion Lumos (Thermo Scientific, USA) and Agilent 1290 (Agilent Technologies, USA), as previously described [52]. A survey scan was performed in the Orbitrap with a resolution of 120000 (fwhm) (m/z 300-1500) followed by collisioninduced dissociation (CID) in the ion trap of the twenty most abundant precursor ions. The threshold value for these precursor ions was set at 25000 counts. The normalized collision energy was set at 35\%, the isolation width at $1.6 \mathrm{Da}$ and the isolation offset at 0.25 Da. Proteome Discoverer 2.2 software was used for processing the raw MS data. MS/MS scans were searched against the protein database of $N$. meningitidis H44/76 (NCBI 909420) with trypsin enzyme specificity and mass tolerance of precursor ions and fragment ions set to $5 \mathrm{ppm}$ and $0.4 \mathrm{Da}$, respectively. Asparagine deamidation and methionine oxidation were set as variable modifications and lysine dimethylation and $\mathrm{N}$-terminal dimethylation as a fixed modification. Peptides were filtered to $<1 \%$ FDR using the Percolator algorithm (Thermo Scientific). Abundance ratios of the proteins were determined with the default dimethyl quantification workflow of Proteome Discoverer using the dimethyl $\left(\mathrm{C}_{1} \mathrm{H}_{3}\right)_{2}(+28.03130)$ and $\left(\mathrm{C}_{1} \mathrm{D}_{2} \mathrm{H}_{1}\right)_{2}(+32.05641)$ channels.

The protein abundance ratios of the biological triplicates were excluded from the dataset if the protein was detected in less than two out of three replicates. This resulted in a dataset of 621 proteins that were detected in all time-points of the samples. The abundance ratios of the proteins were log2 transformed to obtain fold changes and heat maps were generated.

\section{OMV purification}

From the bioreactor cultures, $50 \mathrm{~mL}$ samples were obtained. Samples were centrifuged for 20 minutes at $3000 \times g, 4^{\circ} \mathrm{C}$, to separate bacteria from the OMV containing supernatant. Next, the supernatant was filtered over a $0.2 \mu \mathrm{m}$ filter (Nalgene Rapid Flow, PES) and the sterile OMV fraction was concentrated and washed with one volume of $0.01 \mathrm{M}$ Tris buffer pH 7.4 containing 3\% sucrose on 100 kDa MWCO Amicon Ultra-15 Centrifugal Filter Units according to manufacturer's protocol (Merck Millipore). Lastly, the OMVs were pelleted by ultracentrifugation $\left(125,000 \times g, 2 \mathrm{~h}, 4^{\circ} \mathrm{C}\right)$ and the OMV pellet was resuspended in a $0.01 \mathrm{M}$ Tris buffer $\mathrm{pH} 7.4$ containing $3 \%$ sucrose. 


\section{OMV composition}

Phospholipid and LPS content was analyzed by fatty acid composition measurement using a modified gas chromatography method as described previously [10]. LPS quantification was based on the peak area of C14:30H (two molecules per LPS moiety) using C12:0-30H as internal standard. Phospholipid was quantified by the sum of the peak areas C16:0, C16:1, C18:0 and C18:1 using C15:0 as the internal standard.

\section{Nanoparticle tracking analysis}

To analyze purified sOMVs, a NanoSight NS500 (Malvern Instruments) with $488 \mathrm{~nm}$ wavelength laser module and SCMOS camera was used for nanoparticle tracking analysis (NTA)[53]. A sample was measured in static mode by capturing 10 movies of 30 -seconds with temperature control at $25^{\circ} \mathrm{C}$. Movies were analyzed in the NTA 3.2 software build 3.2.16. The NS500 was calibrated by the NanoSight NTA concentration Measurement upgrade. The sample changer was used to increase the throughput of samples measured [54]. The NS500 is cleaned monthly with Decon 90, following the manufacturers recommendations. Before every set of sample measurements, we confirmed the absence of particles in the MilliQ diluent by measuring the MilliQ diluent for 60 seconds in static mode. 


\section{Results}

\section{Influence of sulfur depletion on SOMV release}

After initial adaptation of $\mathrm{Nm}$ on sulfate, growth on other oxidized sulfur sources such as sulfite and thiosulfate was also possible without the requirement of an additional adaptation phase. Growth in shaker flasks on oxidized sulfur sources was comparable to growth on cysteine (Data not shown). Growth on sulfate, the most oxidized sulfur source was compared to growth on the preferred (reduced) sulfur source cysteine in benchtop bioreactor cultures. The dissolved oxygen tension of the cultures was controlled at $30 \%$ air saturation. The growth pattern of $\mathrm{Nm}$ on sulfate shows exponential growth with an average specific growth rate of $0.47 \mathrm{~h}^{-1}$ until the maximum optical density in this medium is reached (Fig. 1A). The average specific growth rate on cysteine is similar, $0.46 \mathrm{~h}^{-1}$. The carbon dioxide production pattern of the cultivation on both sulfur sources shows the typical pattern of a batch culture, with a respiratory quotient of 1.0 throughout the culture. Previously it was shown that cysteine depletion causes growth arrest [24] and with similar biomass yields on sulfate we assume sulfate limitation is the cause of the onset of the stationary phase of the sulfate based culture. At this point in both cultures, other nutrients like the carbon, nitrogen, and phosphate source are still available in abundance (data not shown). Both cultures show minimal OMV release during the exponential growth phase (Fig. 1B). In the stationary phase both cultures produce sOMVs. However, the sOMV release upon sulfate depletion showed higher than during cysteine depletion.
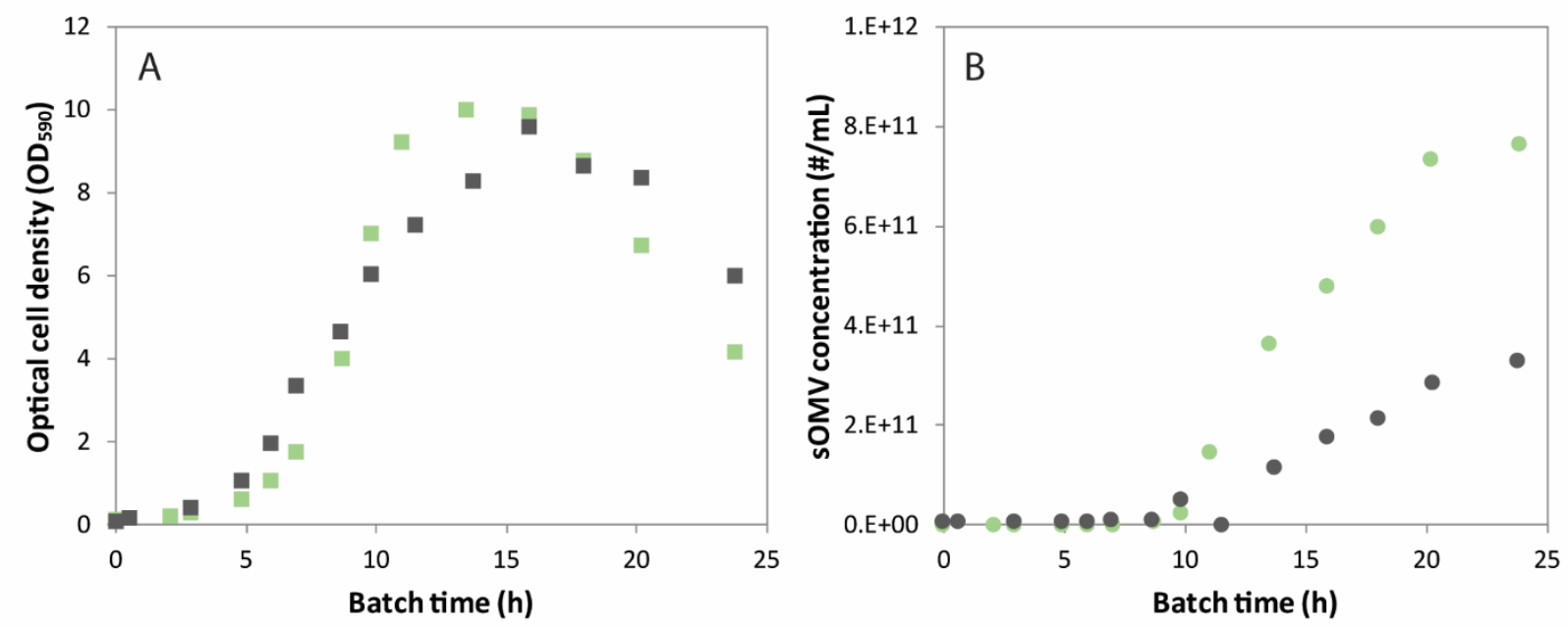

Figure 1 Growth and sOMV release of Nm during sulfur source depletion. Nm cultures are grown on cysteine (black) and sulfate (green) as sulfur source. The growth curve based on optical density is shown in graph $\mathbf{A}$, and the concentration of OMV is given in graph $\mathbf{B}$. Graphs are the overlay of two replicate cultures to practically allow for sufficient data points covering $24 \mathrm{~h}$. 


\section{Proteome analysis of cysteine and sulfate depleted batch cultures}

To understand how the bacterium copes with the onset of sulfur depletion during cultivation the proteome was examined. Triplicate bioreactor cultures with either cysteine or sulfate as sole sulfur source were analyzed at different time-points during the cultivation (Fig. 2A). Culture supernatants showed depletion of the corresponding sulfur source at 7h (Fig. 2A, time-point t2). Sulfur depletion results in increased OMV concentrations in the culture (Fig. 2B), confirming the previous results (Fig. 1). During growth in the presence of cysteine (t1), the proteome of $\mathrm{Nm}$ shows no upregulation of the cysteine biosynthesis pathway as expected as cysteine is sufficiently available (Fig. 2C, Supplemental File 1 for the complete dataset). The pathway is also not upregulated

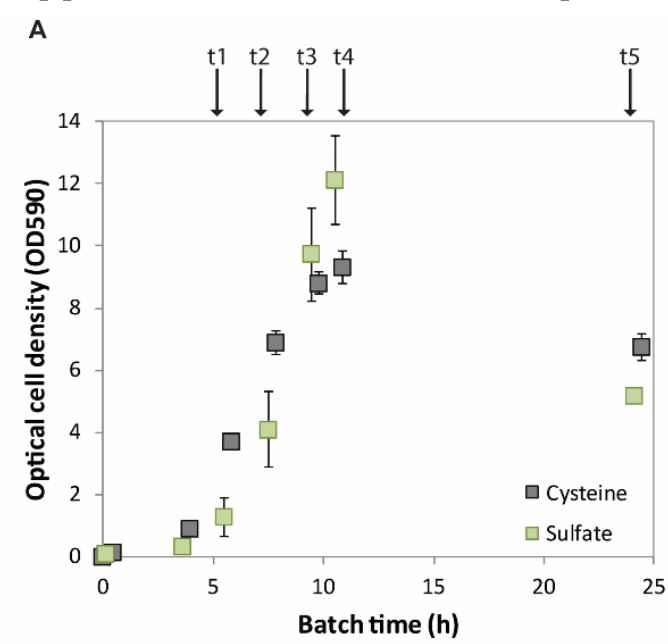

B
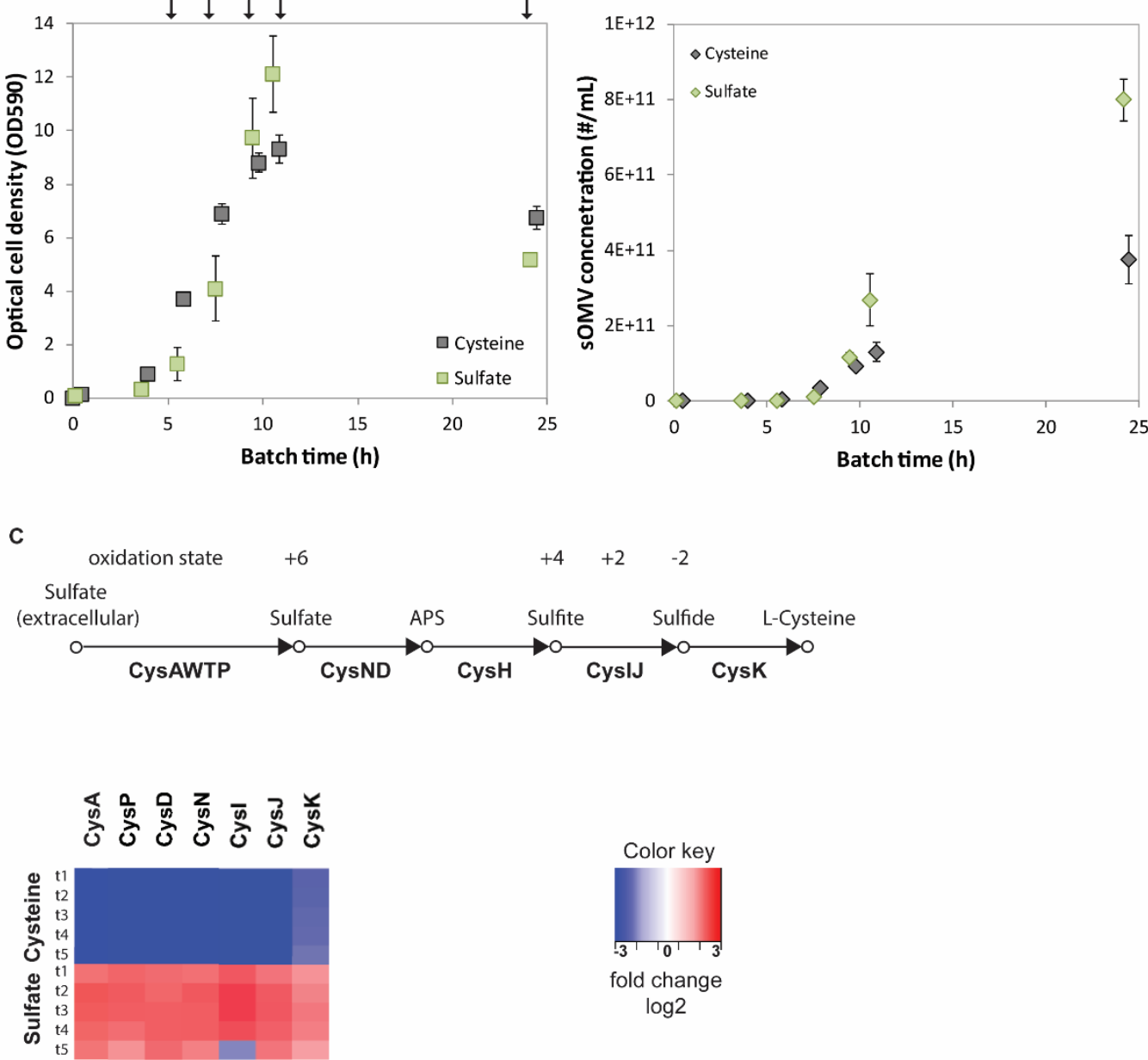

Figure 2. Experimental approach for proteomics. Triplicate $\mathrm{Nm}$ batch cultures on cysteine or sulfate as the sole sulfur source were sampled during exponential growth $(\mathrm{t} 1, \mathrm{t} 2)$, onset stationary phase $(\mathrm{t} 3, \mathrm{t} 4)$, and late stationary phase (t5)(A). The OMV concentration was measured by NTA throughout the cultivation (B). Error bars indicate standard deviation from the mean. The proteome is assessed for the cysteine assimilation pathway as shown in the heat map (C). 
when cysteine becomes limiting ( $\mathrm{t} 3-\mathrm{t} 5)$, possibly because no sulfur is available for the generation of new proteins, or since no other sulfur source is available. CysW, CysT and CysH were below the detection limit of the LC-MS/MS analysis. During growth on sulfate the cysteine biosynthesis pathway was upregulated throughout the cultivation as expected.

We then performed gene ontology enrichment on the proteome data. Proteins categorized in the gene ontology antioxidant activity (G0:16209) were found to be upregulated upon cysteine depletion (time points $\mathrm{t} 3$, t4) (Fig. 3). This agrees to the previously described antioxidant and redox-stress responses found in the transcriptome upon cysteine depletion [24]. Upon sulfate depletion proteins with antioxidant activity are upregulated as well. Upon cysteine depletion, AhpD and AhpC are upregulated. Upon sulfate depletion these appear not to be regulated, while another protein with ascribed alkylhydroxyperoxidase activity (ahpD, E6MUC5) was observed to be regulated on sulfate as sulfur source. Proteins involved in phospholipid (G0:8654), fatty acid and LPS production (G0:9103) were upregulated after both cysteine depletion and sulfate depletion, simultaneously with increased OMV release. Both phospholipid biosynthesis and fatty acid biosynthesis proteins were more upregulated after sulfate depletion than after cysteine depletion. This upregulation is consistent with the increased release of OMV observed after sulfate depletion. Interestingly, the VacJ/Yrb ABC (ATP-binding cassette) transporter, which has a proposed role in maintaining lipid asymmetry in the outer membrane by transport of phospholipids from the outer leaflet to the inner leaflet, is strongly upregulated upon growth on sulfate as sulfur source and is not regulated upon cysteine depletion.

\section{Sulfur depletion results in phospholipid enriched OMVs}

During OMV production as a result of sulfur depletion, increased phospholipid production takes place, which could result in OMVs enriched in phospholipid content. 
Antioxidant activity

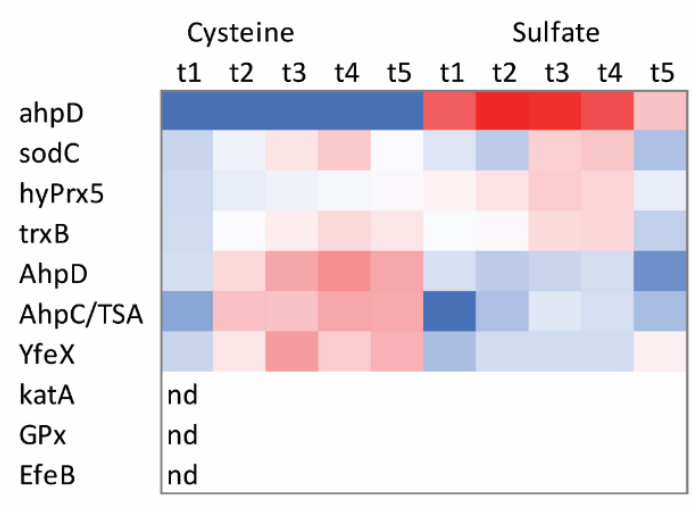

Fatty acid biosynthetic process Cysteine Sulfate

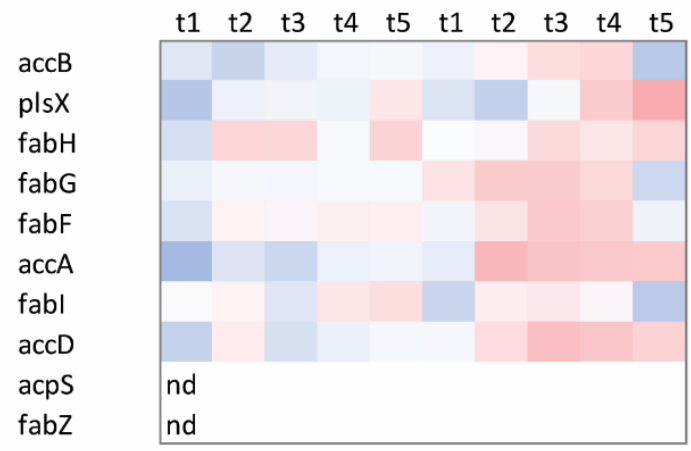

LPS biosynthetic process

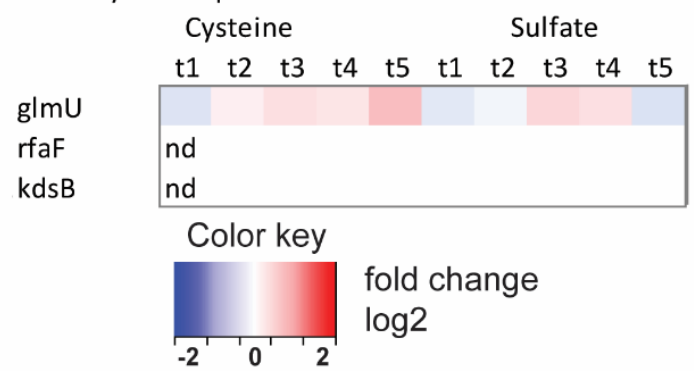

phospholipid biosynthetic process

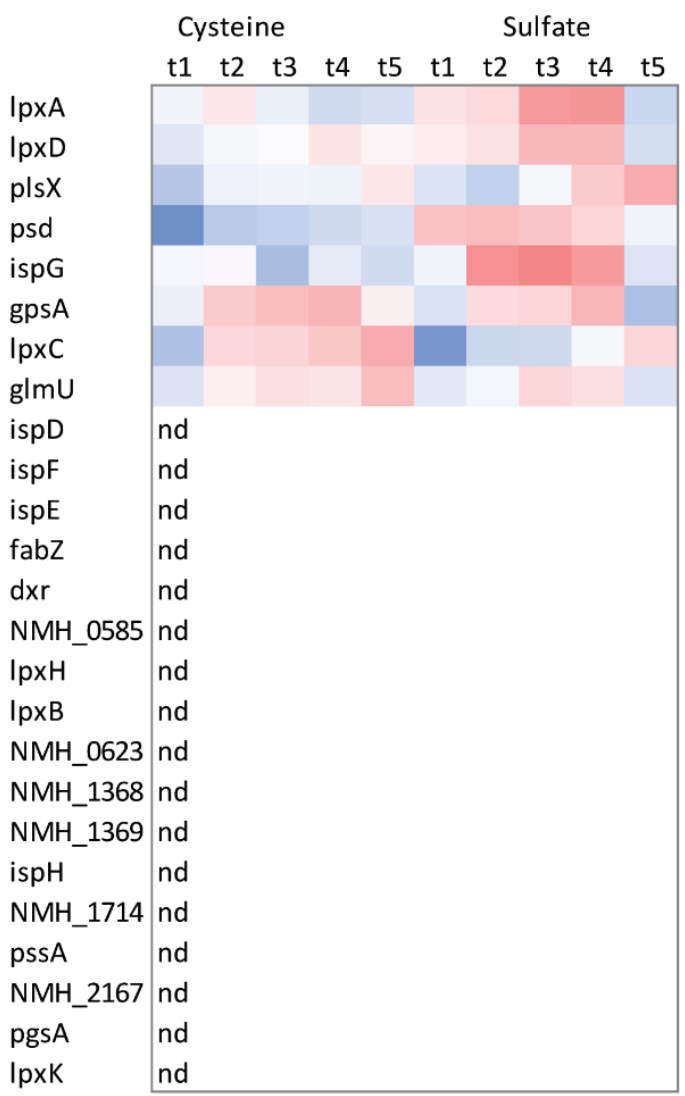

VacJ/Yrb ABC transport system

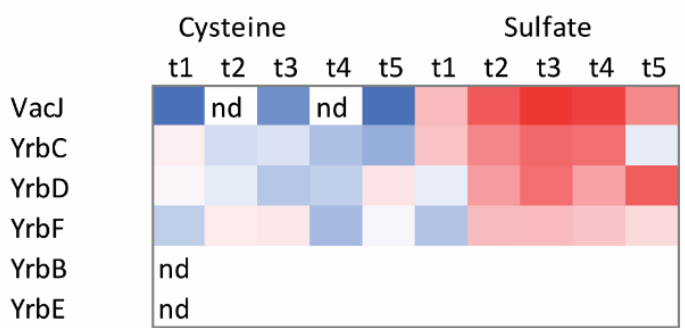

Figure 3. Heat maps based on gene ontology. Heat maps for specific gene ontology groups from the proteome dataset. The gene ontologies for antioxidant activity (G0:16209), Fatty acid biosynthesis (G0:6633), LPS biosynthesis (G0:9103), and phospholipid biosynthesis (G0:8654) are shown. Additionally, the expression level is shown of the VacJ/Yrb ABC transport system. VacJ was included although it was only detected in 8 out of 10 time-points, since the VacJ levels were near the detection limit in the cysteine culture. Samples indicated by "nd" were not detected.

This changed composition of the OMVs could also affect their size. To test this, OMVs were isolated from batch cultures containing either sulfate or cysteine, during the exponential growth phase, onset of the stationary phase and in the stationary phase. The size of OMVs was measured by NTA and was found to be similar throughout the cultivation and between sulfate depletion and cysteine depletion (Fig. 4A). A small trend 
in increased OMV size upon sulfur depletion was observed, although non-significant. The phospholipid content of OMVs increased upon sulfate depletion, while upon cysteine depletion the phospholipid content only shows a minor increase (Fig. 4B). The relative content of LPS, based on fatty acid analysis, decreased similarly for both sulfate and cysteine depletion (Fig. 4C).

\section{Discussion}

This study shows that not cysteine depletion specific, but sulfur depletion in general induces OMV release in Nm cultures. Growth of Nm on sulfate was comparable to growth on cysteine as sulfur source. Upon sulfur depletion, the proteome showed increases in antioxidant activity, phospholipid biosynthesis and LPS biosynthesis. OMVs released after sulfur depletion were enriched in phospholipids. The most interesting finding was that sulfate depleted cultures showed increased OMV release over cysteine depleted cultures.

OMV formation upon sulfur depletion can be the result of increased phospholipid biosynthesis. Especially OMVs produced after sulfate depletion showed to be enriched in phospholipids. This finding was supported by the increased phospholipid biosynthesis observed in the proteome. Recently, an important role was shown for the
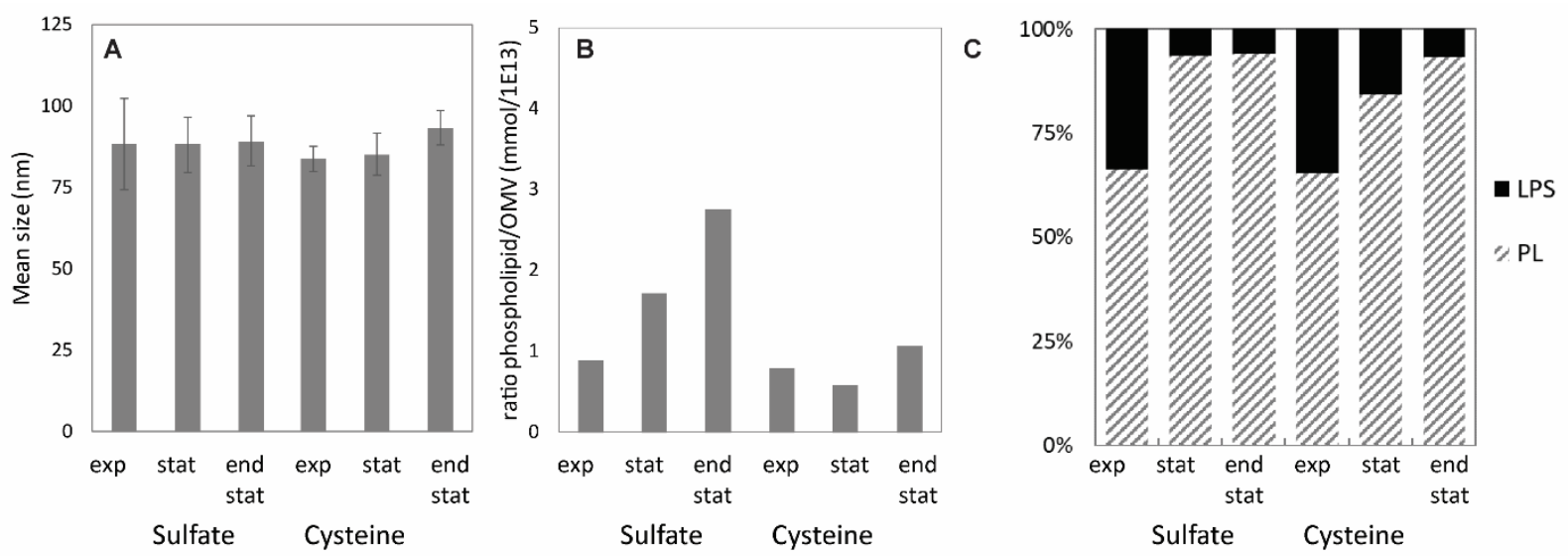

Figure 4. OMV characteristics upon sulfur depletion. OMV size by Nanoparticle Tracking Analysis of purified sOMV at different growth stages of the batch cultures on cysteine and sulfate (A). Error bars indicate standard deviation of the mean of 10 measurements. The biochemical composition of OMVs produced upon sulfur depletion show increased phospholipid/OMV ratio (B). The fatty acid distribution between phospholipid and LPS is shown upon sulfur source depletion (C). 
Vac]/Yrb ABC (ATP-binding cassette) transporter in OMV biogenesis [16]. This transporter has a proposed role in maintaining lipid asymmetry in the outer membranes as phospholipid transporter [32], by transporting phospholipids from the outer leaflet of the membrane to the inner leaflet, and is annotated in the Nm genome. It was shown by Roier et al. that the VacJ/Yrb ABC transporter was downregulated by the ferric uptake regulator (FUR) upon iron limitation in H. influenzae, V. cholerae and E. coli [16]. Disruptions of the VacJ/Yrb ABC transport system resulted in increased OMV formation, due to phospholipid accumulation in the outer membrane. This increase in OMV production in $H$. influenzae was not accompanied with increased fatty acid biosynthesis. In this study we show that sulfur depletion causes increased release of OMVs that are enriched in phospholipids. It should be noted that we used a strain with reduced linkage between the peptidoglycan and the outer membrane ( $r m p M$ knockout mutant). Interestingly, our Nm proteomics data upon sOMV formation by sulfate depletion showed simultaneous upregulation of the VacJ/Yrb ABC transporter and phospholipid biosynthesis. Possibly, this transporter is upregulated to counteract the accumulation of phospholipids in the outer membrane due to the increased synthesis and stop of growth. The overproduction of OMVs could be a method to dispose of the excess phospholipids in the outer membrane because the transporter has insufficient capacity. This suggests that upon sulfur depletion, OMV release is triggered in a different manner than downregulation of the VacJ/Yrb ABC transport system. Although it does support the OMV biogenesis model of phospholipid accumulation based OMV release [19].

The increased phospholipid production upon growth on sulfate can be a result of the altered redox state. During cysteine assimilation from sulfate, NADPH is required to reduce the oxidized sulfur source [30]. NADPH replenishment is thus higher during growth on sulfate, than for growth on cysteine. We hypothesize that upon sulfate depletion the high NADPH replenishment causes a surplus of NADPH since no NADPH is used for cysteine assimilation anymore. Phospholipid production, which also requires $\mathrm{NAPDH}$, is then increased as a sink for the NAPDH that is produced in excess. As NADPH is one of the key cofactors in the metabolic network and influenced by many reactions [33] it can be argued whether a short perturbation causes a prolonged state of OMV release. After sulfur depletion, increased levels of serine could be expected since no sulfide is available anymore to produce cysteine. Besides conversion to cysteine, serine 
can be used for the production of phospholipids by the conversion to phosphatidylserine. Engineering the redox metabolism is a method for enhanced lipid production in microbes [34]. For example, lipid production in Yarrowia lipolytica has been boosted by introduction of pathways that convert NADH to NADPH [35]. Another important role of NADPH is as cofactor of enzymes involved in oxidative stress responses as catalase, superoxide dismutase and glutathione peroxidase [36]. Alternatively, the increased phospholipid production can be required to maintain the membrane integrity because of the increased OMV release. Moreover, sulfur depletion could stimulate OMV release through a secondary mechanism such as oxidative stress. Sulfur is an important component of major oxidative stress responses [37] and sulfur starvation may induce OMV release by affecting these oxidative stress responses. Oxidative stress responses were observed in the gene expression profile after cysteine depletion previously [38]. As sulfur was unavailable, glutathione biogenesis and ironsulfur protein biogenesis was impaired. Biologically, Nm encounters sudden oxidative stress in the oxidative burst that phagocytes apply to eliminate invading pathogens [37, 39]. Increased OMV release upon oxidative stress can be a method to release oxidative stress or a method to enhance survival upon oxidative bursts.

OMV formation as a result of nutrient limitation has been posed as an evolutionary trigger for OMV release as survival is enhanced through nutrient scavenging [40]. Recently, amino acid deprivation was found to stimulate OMV and tubular OMV structures in Francisella tularensis [41]. Nm OMVs have been found associated with iron-scavenging proteins [42] and Bacteroides succinogenes OMVs have been found to contain xylanases or cellulases that can assist in nutrient acquisition [43]. OMV formation upon nutrient limitation may thus assist in nutrient acquisition and improve bacterial survival.

The size of OMVs was similar for OMVs produced after depletion of sulfate and cysteine, while OMV size can be highly subjectable to changes. The induction of OMV release of Pseudomonas aeruginosa by gentamycin yields enlarged OMVs [15, 44]. Mitomycin C treatment of Shigella dysenteriae induces the release of larger OMVs [45]. The size of OMVs can affect both the production process of OMVs, as it influences purification and analysis of OMVs, and the immunogenicity as the size influences the uptake by antigen presenting cells [46]. The observed similarity in size distribution of OMVs in this study 
is somewhat surprising as the vesicles produced upon sulfate depletion where enriched in phospholipids. This results in a lower content of LPS, which could affect the use of these OMVs as vaccines. The biological activity of native Nm LPS is high, as characterized by its activation of the TLR4/MD-2 complex. LPS can be engineered such, by changes in lipid A acylation and phosphorylation, that these LPS derivates have various strengths of TLR4/MD-2 activation [47]. Future work should tune the required LPS activity of these spontaneously released OMVs with reduced LPS content to optimize the amount of immune activation while limiting toxic side effects for an optimal vaccine composition.

Taken together, the findings of this study expand the knowledge on OMV release by $\mathrm{Nm}$. Sulfur depletion can be used as general mechanism to trigger vesicle formation in $\mathrm{Nm}$ and the use of sulfate as sulfur source improves the OMV productivity of $\mathrm{Nm}$ batch cultures. Sulfur depletion caused overproduction of phospholipids and especially sulfate depletion resulted in OMVs enriched in phospholipid content. OMV biogenesis remains a complicated and poorly understood process that leaves many research questions remaining. Refined understanding of OMV biogenesis will boost production processes of OMVs released in the supernatant of bacterial cultures and will ultimately make OMV extraction processes obsolete for the production of OMV-based vaccines.

\section{Acknowledgements}

The authors thank Tim Bindels for the genetic modification of the Nm strains, Alex de Haan and Martin Hamzink for GC measurements and performing nutrient analysis, and René Raeven and Ronald Maas for the helpful discussions. This work has been funded by the Ministry for Health, Welfare and Sports (The Netherlands).

\section{References}

1. Kulp, A. and M.J. Kuehn, Biological functions and biogenesis of secreted bacterial outer membrane vesicles. Annu Rev Microbiol, 2010. 64: p. 163-84.

2. Holst, J., et al., Properties and clinical performance of vaccines containing outer membrane vesicles from Neisseria meningitidis. Vaccine, 2009. 27 Suppl 2: p. B3-12.

3. Bjune, G., et al., Effect of outer membrane vesicle vaccine against group B meningococcal disease in Norway. Lancet, 1991. 338(8775): p. 1093-6.

4. Sierra, G.V., et al., Vaccine against group B Neisseria meningitidis: protection trial and mass vaccination results in Cuba. NIPH Ann, 1991. 14(2): p. 195-207; discussion 208-10.

5. Thornton, V., et al., Safety and immunogenicity of New Zealand strain meningococcal serogroup B OMV vaccine in healthy adults: beginning of epidemic control. Vaccine, 2006. 24(9): p. 1395-400. 
6. Donnelly, J., et al., Qualitative and quantitative assessment of meningococcal antigens to evaluate the potential strain coverage of protein-based vaccines. Proc Natl Acad Sci U S A, 2010. 107(45): p. 194905.

7. van der Pol, L., M. Stork, and P. van der Ley, Outer membrane vesicles as platform vaccine technology. Biotechnol J, 2015. 10(11): p. 1689-706.

8. van der Ley, P., et al., Modification of lipid A biosynthesis in Neisseria meningitidis lpxL mutants: influence on lipopolysaccharide structure, toxicity, and adjuvant activity. Infect Immun, 2001. 69(10): p. 5981-90.

9. Zariri, A. and P. van der Ley, Biosynthetically engineered lipopolysaccharide as vaccine adjuvant. Expert Rev Vaccines, 2015. 14(6): p. 861-76.

10. van de Waterbeemd, B., et al., Improved $\mathrm{OMV}$ vaccine against Neisseria meningitidis using genetically engineered strains and a detergent-free purification process. Vaccine, 2010. 28(30): p. 4810-6.

11. van de Waterbeemd, B., et al., Identification and optimization of critical process parameters for the production of NOMV vaccine against Neisseria meningitidis. Vaccine, 2012. 30(24): p. 3683-90.

12. Lee, E.Y., et al., Global proteomic profiling of native outer membrane vesicles derived from Escherichia coli. Proteomics, 2007. 7(17): p. 3143-53.

13. McBroom, A.J. and M.J. Kuehn, Release of outer membrane vesicles by Gram-negative bacteria is a novel envelope stress response. Mol Microbiol, 2007. 63(2): p. 545-58.

14. Haurat, M.F., et al., Selective sorting of cargo proteins into bacterial membrane vesicles. J Biol Chem, 2011. 286(2): p. 1269-76.

15. Kadurugamuwa, J.L. and T.J. Beveridge, Virulence factors are released from Pseudomonas aeruginosa in association with membrane vesicles during normal growth and exposure to gentamicin: a novel mechanism of enzyme secretion. J Bacteriol, 1995. 177(14): p. 3998-4008.

16. Roier, S., et al., A novel mechanism for the biogenesis of outer membrane vesicles in Gram-negative bacteria. Nat Commun, 2016. 7: p. 10515.

17. Kaparakis-Liaskos, M. and R.L. Ferrero, Immune modulation by bacterial outer membrane vesicles. Nat Rev Immunol, 2015. 15(6): p. 375-87.

18. Kulp, A.J., et al., Genome-Wide Assessment of Outer Membrane Vesicle Production in Escherichia coli. PLoS One, 2015. 10(9): p. e0139200.

19. Volgers, C., P.H.M. Savelkoul, and F.R.M. Stassen, Gram-negative bacterial membrane vesicle release in response to the host-environment: different threats, same trick? Critical Reviews in Microbiology, 2018. 44(3): p. 258-273.

20. Bernadac, A., et al., Escherichia coli tol-pal mutants form outer membrane vesicles. J Bacteriol, 1998. 180(18): p. 4872-8.

21. Sonntag, I., et al., Cell envelope and shape of Escherichia coli: multiple mutants missing the outer membrane lipoprotein and other major outer membrane proteins. J Bacteriol, 1978. 136(1): p. 280-5.

22. Steeghs, L., et al., Expression of foreign LpxA acyltransferases in Neisseria meningitidis results in modified lipid A with reduced toxicity and retained adjuvant activity. Cell Microbiol, 2002. 4(9): p. 599611.

23. Deatherage, B.L., et al., Biogenesis of bacterial membrane vesicles. Mol Microbiol, 2009. 72(6): p. 1395407.

24. van de Waterbeemd, B., et al., Cysteine depletion causes oxidative stress and triggers outer membrane vesicle release by Neisseria meningitidis; implications for vaccine development. PLoS One, 2013. 8(1): p. e54314.

25. Frantz, I.D., Growth Requirements of the Meningococcus. J Bacteriol, 1942. 43(6): p. 757-61.

26. Grossowicz, N., Growth Requirements and Metabolism of Neisseria intracellularis. J Bacteriol, 1945. 50(1): p. 109-15.

27. Jyssum, K., Assimilation of nitrogen in meningococci grown with the ammonium ion as sole nitrogen source. Acta Pathol Microbiol Scand, 1959. 46: p. 320-32.

28. Port, J.L., I.W. DeVoe, and F.S. Archibald, Sulphur acquisition by Neisseria meningitidis. Can J Microbiol, 1984. 30(12): p. 1453-7.

29. Wesley Catlin, B., Nutritional Profiles of Neisseria gonorrhoeae, Neisseria meningitidis, and Neisseria lactamica in Chemically Defined Media and the Use of Growth Requirements for Gonococcal Typing. The Journal of Infectious Diseases, 1973. 128(2): p. 178-194.

30. Hicks, J.L. and C.V. Mullholland, Cysteine biosynthesis in Neisseria species. Microbiology, 2018.

31. Rusniok, C., et al., NeMeSys: a biological resource for narrowing the gap between sequence and function in the human pathogen Neisseria meningitidis. Genome Biol, 2009. 10(10): p. R110. 
32. Malinverni, J.C. and T.J. Silhavy, An ABC transport system that maintains lipid asymmetry in the Gramnegative outer membrane. Proceedings of the National Academy of Sciences, 2009. 106(19): p. 80098014.

33. Xu, J.-Z., H.-K. Yang, and W.-G. Zhang, NADPH metabolism: a survey of its theoretical characteristics and manipulation strategies in amino acid biosynthesis. Critical Reviews in Biotechnology, 2018. 38(7): p. 1061-1076.

34. Marella, E.R., et al., Engineering microbial fatty acid metabolism for biofuels and biochemicals. Current Opinion in Biotechnology, 2018. 50: p. 39-46.

35. Qiao, K., et al., Lipid production in Yarrowia lipolytica is maximized by engineering cytosolic redox metabolism. Nature Biotechnology, 2017. 35: p. 173.

36. Ying, W., NAD+/NADH and NADP+/NADPH in cellular functions and cell death: regulation and biological consequences. Antioxid Redox Signal, 2008. 10(2): p. 179-206.

37. Seib, K.L., et al., Defenses against oxidative stress in Neisseria gonorrhoeae and Neisseria meningitidis: distinctive systems for different lifestyles. J Infect Dis, 2004. 190(1): p. 136-47.

38. !!! INVALID CITATION !!! \{\}.

39. Moslen, M.T., Reactive oxygen species in normal physiology, cell injury and phagocytosis. Advances in experimental medicine and biology, 1994. 366: p. 17-27.

40. Ellis, T.N. and M.J. Kuehn, Virulence and Immunomodulatory Roles of Bacterial Outer Membrane Vesicles. Microbiology and Molecular Biology Reviews, 2010. 74(1): p. 81-94.

41. Sampath, V., W.D. McCaig, and D.G. Thanassi, Amino acid deprivation and central carbon metabolism regulate the production of outer membrane vesicles and tubes by Francisella. Molecular Microbiology, 2017. 107(4): p. 523-541.

42. Lappann, M., et al., Comparative proteome analysis of spontaneous outer membrane vesicles and purified outer membranes of Neisseria meningitidis. Journal of Bacteriology, 2013.

43. Forsberg, C.W., T.J. Beveridge, and A. Hellstrom, Cellulase and Xylanase Release from Bacteroides succinogenes and Its Importance in the Rumen Environment. Applied and Environmental Microbiology, 1981. 42(5): p. 886-896.

44. Sabra, W., H. Lunsdorf, and A.P. Zeng, Alterations in the formation of lipopolysaccharide and membrane vesicles on the surface of Pseudomonas aeruginosa PA01 under oxygen stress conditions. Microbiology, 2003. 149(Pt 10): p. 2789-95.

45. Dutta, S., et al., Release of Shiga Toxin by Membrane Vesicles in Shigella dysenteriae Serotype 1 Strains and In Vitro Effects of Antimicrobials on Toxin Production and Release. Microbiology and Immunology, 2004. 48(12): p. 965-969.

46. Gerritzen, M.J.H., et al., Bioengineering bacterial outer membrane vesicles as vaccine platform. Biotechnol Adv, 2017. 35(5): p. 565-574.

47. Zariri, A., et al., Modulating endotoxin activity by combinatorial bioengineering of meningococcal lipopolysaccharide. Scientific Reports, 2016. 6: p. 36575.

48. Holten, E., Serotypes of Neisseria meningitidis isolated from patients in Norway during the first six months of 1978. J Clin Microbiol, 1979. 9(2): p. 186-8.

49. Steeghs, L., et al., Neisseria meningitidis expressing lgtB lipopolysaccharide targets DC-SIGN and modulates dendritic cell function. Cell Microbiol, 2006. 8(2): p. 316-25.

50. Baart, G.J., et al., Modeling Neisseria meningitidis metabolism: from genome to metabolic fluxes. Genome Biol, 2007. 8(7): p. R136.

51. van de Waterbeemd, B., et al., Quantitative Proteomics Reveals Distinct Differences in the Protein Content of Outer Membrane Vesicle Vaccines. Journal of Proteome Research, 2013. 12(4): p. 18981908.

52. Meiring, H.D., et al., Nanoscale LC-MS(n): technical design and applications to peptide and protein analysis. Journal of Separation Science, 2002. 25(9): p. 557-568.

53. Malloy, A. and B. Carr, NanoParticle Tracking Analysis - The Halo ${ }^{\mathrm{Tm}}$ System. Particle \& Particle Systems Characterization, 2006. 23(2): p. 197-204.

54. Gerritzen, M.J.H., et al., High throughput nanoparticle tracking analysis for monitoring outer membrane vesicle production. Journal of Extracellular Vesicles, 2017. 6(1): p. 1333883. 


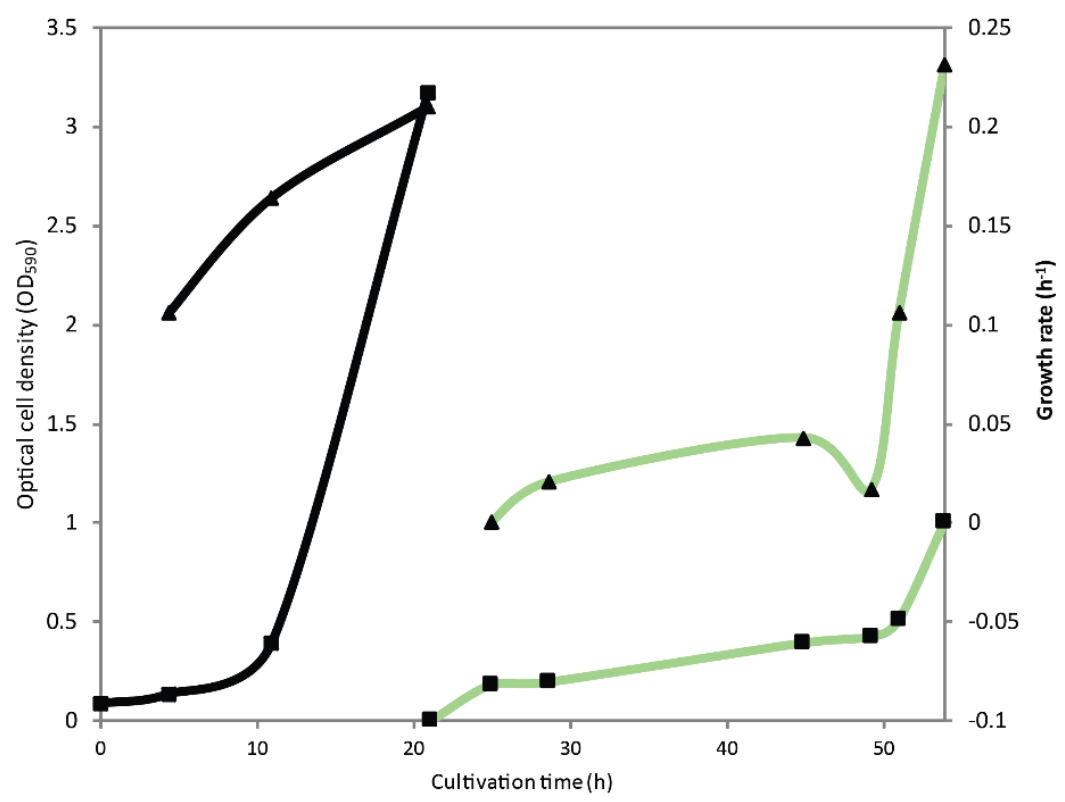

Supplemental Figure 1. Adaptation of Neisseria meningitidis from cysteine to sulfate as sulfur source. Optical density is shown by the square markers, and the growth rate by the triangular markers. Growth on cysteine medium is shown in black and growth on sulfate medium in green. A culture inoculated from a frozen seed was grown on cysteine containing medium $(0 \mathrm{~h}-20 \mathrm{~h})$. Next this culture was subcultured to medium with sulfate. A period of adaptation $(22 \mathrm{~h}-48 \mathrm{~h})$ was required until growth is observed.

\section{Supplemental File 1 Dataset proteomics (Dataset proteomics.xlsx)}




\section{Chapter 5}

\section{High dissolved oxygen tension triggers outer membrane vesicle formation by Neisseria meningitidis}

Published as:

Gerritzen, M.J.H., R.H.W. Maas, J. van den IJssel, L. van Keulen, D.E. Martens, R.H. Wijffels and M. Stork (2018). "High dissolved oxygen tension triggers outer membrane vesicle formation by Neisseria meningitidis."

Microbial Cell Factories, 2018. 17(1): p. 157. 


\begin{abstract}
Outer membrane vesicles (OMVs) are nanoparticles released by Gram-negative bacteria and can be used as vaccines. Often, detergents are used to promote release of OMVs and to remove the toxic lipopolysaccharides. Lipopolysaccharides can be detoxified by genetic modification such that vesicles spontaneously produced by bacteria can be directly used as vaccines. The use of spontaneous OMVs has the advantage that no separate extraction step is required in the purification process. However, the productivity of spontaneous OMVs by bacteria at optimal growth conditions is low. One of many methods for increasing OMV formation is to reduce the linkage of the outer membrane to the peptidoglycan layer by knocking out the rmpM gene. A previous study showed that for Neisseria meningitidis this resulted in release of more OMVs. Furthermore, cysteine depletion was found to trigger OMV release and at the same time cause reduced growth and oxidative stress responses. Here we study the effect of growth rate and oxidative stress on OMV release. First, we identified using chemostat and accelerostat cultures of $N$. meningitidis that increasing the growth rate from $0.03 \mathrm{~h}^{-1}$ to $0.18 \mathrm{~h}^{-1}$ has a limited effect on OMV productivity. Thus, we hypothesized that oxidative stress is the trigger for OMV release and that oxidative stress can be introduced directly by increasing the dissolved oxygen tension of bacterial cultures. Slowly increasing oxygen concentrations in a N. meningitidis changestat showed that an increase from $30 \%$ to $150 \%$ air saturation improved OMV productivity four-fold. Batch cultures controlled at $100 \%$ air saturation increased OMV productivity three-fold over batch cultures controlled at $30 \%$ air saturation. Increased dissolved oxygen tension induces the release of outer membrane vesicles in $N$. meningitidis cultures. Since oxygen concentration is a well-controlled process parameter of bacterial cultures, this trigger can be applied as a convenient process parameter to induce OMV release in bacterial cultures. Improved productivity of OMVs not only improves the production costs of OMVs as vaccines, it also facilitates the use of OMVs as adjuvants, enzyme carriers, or cell-specific drug delivery vehicles.
\end{abstract}




\section{Introduction}

Outer membrane vesicles (OMVs) are naturally produced by Gram-negative bacteria and play a role in pathogenesis, cell-to-cell communication and stress responses [1]. OMVs are spherical nanoparticles that consist of a phospholipid bilayer with proteins and lipopolysaccharides (LPS) [1]. The lumen of the vesicle contains DNA and periplasmic components of the bacterium [2, 3]. Membrane vesicle formation has been shown recently in Gram-positive bacteria and archaea as well [4, 5].

OMVs are highly similar to the outer membrane of the bacteria, are non-replicating, and characteristically are full of pathogen associated molecular patterns. With this they fulfill major criteria for vaccine design and have been successfully used as such [6, 7]. These vaccines have been produced by extraction of vesicles from the bacterial outer membrane using detergents. In this way, vesicles are artificially formed and the amount of toxic LPS could be reduced $[8,9]$. However, extraction of vesicles is disadvantageous since the proteome of extracted OMVs (eOMVs) shows a lowered amount of possible immunogenic proteins over spontaneously released OMVs (sOMVs) [10,11].

Furthermore, extraction methods are not required anymore for LPS removal since the introduction of genetically modified low toxicity LPS $[12,13]$, which forms the basis for the use of spontaneously released OMV. Thus, the use of spontaneous released vesicles simplifies the purification of OMVs since it obsoletes the extraction step in the downstream processing of the vaccine product $[10,11]$. Sera from mice immunized with spontaneous OMVs show immunity against a broader range of serotypes than mice immunized with detergent extracted OMVs [14]. Furthermore, omission of detergent also preserves vesicle integrity, yielding a more uniform vaccine product.

Feasible sOMV production has not been straightforward since sOMV productivity at optimal growth conditions is low. Despite the research on OMV biogenesis over the past 4 decades, the exact mechanism triggering the release of OMVs by a bacterium remains unknown. Because the composition of OMVs differs from the outer membrane of the bacteria, it is generally thought that the release of vesicles is not a random process [15]. Biogenesis of OMVs has been described by several models although it remains unclear whether a shared mechanism exists [16]. OMV biogenesis is hypothesized to be based on peptidoglycan fragments accumulation in the periplasm, less anchoring of the outer membrane to the peptidoglycan layer, or O-antigen charge repulsion. These models are 
reviewed in [17] and [2]. Recently, Roier et al. suggested a novel mechanism based on phospholipid accumulation that is conserved among Gram-negative bacteria [18]. The proposed phospholipid transporter VacJ/Yrb ATP-binding cassette was shown to be involved in OMV production and could be part of regulated OMV release. Increasing the OMV production by Neisseria meningitidis is possible by deleting the rmpM gene, the product of which anchors the outer membrane to the peptidoglycan layer [14].

Reducing the linkage between the outer membrane and the peptidoglycan layer results in so-called blebbing mutants of bacteria that show increased release of OMVs in the supernatant. This was found not only for $N$. meningitidis, but also for E. coli [19].

External triggers for OMV release could be a convenient way to enhance production in bioreactor cultures. Van de Waterbeemd et al. showed that cysteine depletion can be used as a trigger to stimulate the release of vesicles in N. meningitidis cultures [20]. Simultaneously with cysteine depletion, the growth rate is reduced and oxidative stress responses were observed in the transcriptome of the bacterium. It is unknown whether cysteine directly triggers OMV formation or works indirectly through a reduction in growth rate and/or increase in oxidative stress. Furthermore, increased release of vesicles under hydrogen peroxide addition has been shown [20]. The method of hydrogen peroxide addition, however, is not feasible for scalable production processes of OMV since local hydrogen peroxide addition to a bacterial culture will result in significant cell death and lysis. In this study we hypothesize that extracellular oxidative stress is directly induced by high concentrations of dissolved oxygen, which is one of the controlled parameters in bioreactor cultivations. The dissolved oxygen tension is typically kept low, to minimize the stress from hyperoxia and to prevent oxygen inhibition [21]. Especially for a facultative anaerobic pathogen it is obvious to design the cultivation with low oxygen concentration [22]. For example, N. meningitidis cultivation for both the vaccine concepts HexaMen and NonaMen has been designed with levels of $30 \%$ air saturation $[23,24]$.

The aim of this study is to obtain more insight in the role of growth rate and oxidative stress in the release of OMVs. The first section of this paper will examine whether a decrease in growth rate can trigger OMV release by using accelerostat experiments. Next, oxidative stress is introduced in continuous cultures by increasing the dissolved 
oxygen tension. Lastly, increasing the dissolved oxygen concentration is tested on batch cultures.

\section{Material and methods}

\section{Bacterial strains}

A recombinant derivate of the Neisseria meningitidis serogroup B isolate H44/76 [25] was used in this study. The selected strain was a PorA lacking derivate of the H44/76 isolate [26]. This strain has a non-encapsulated phenotype due to the siaD knockout, IpxL1 deletion to attenuate LPS-toxicity, rmpM deletion to improve vesicle formation (unless indicated otherwise) and $\lg t B$ mutation to promote interactions with dendritic cells $[14,27]$. This strain was stored in glycerol as working seedlots. All cultivations were performed in chemically defined growth medium [28].

For the cultivation with Escherichia coli strain JC8031 (TolRA) was used [29]. A shaker flask culture was started by adding $10 \mu \mathrm{L}$ of frozen glycerol stock $\left(-80^{\circ} \mathrm{C}\right)$ to $100 \mathrm{~mL} \mathrm{LB}$ medium (Large Capsules: tryptone $10 \mathrm{~g} / \mathrm{L}$, yeast extract $5 \mathrm{~g} / \mathrm{L}, \mathrm{NaCl} 10 \mathrm{~g} / \mathrm{L}, \mathrm{MP}$ Biomedicals) and incubating the shaker flask at $37^{\circ} \mathrm{C}$ for 16 hours. Bioreactor cultivations were performed on LB medium without antifoam with a maximum stirrer speed of $600 \mathrm{RPM}$ at $37^{\circ} \mathrm{C}$.

\section{Bioreactor cultivations}

Batch cultivations were performed in 5-liter dished bottom Applikon bioreactors with an H/D ratio of 1.6 based on total volume. Cultivations were operated with 3 liter working volume on a Pierre Guerin Tryton ${ }^{i}$ controller. Temperature was controlled at $35 \pm 0.5^{\circ} \mathrm{C}$ and $\mathrm{pH}$ was controlled at $\mathrm{pH} 7.2 \pm 0.05$ using $1 \mathrm{M} \mathrm{HCl}$ and $1 \mathrm{M} \mathrm{NaOH}$. Dissolved oxygen tension was controlled at 30\% unless indicated otherwise. The membrane covered polarographic oxygen sensor (InPro 6850i, Mettler Toledo) was calibrated at $100 \%$ in air-saturated sterile growth medium of $35^{\circ} \mathrm{C}$. In the first phase of the cultivation, the dissolved oxygen tension is controlled by increasing the agitation rate (300 - $1000 \mathrm{RPM}$ ) followed by increasing the fraction of oxygen in the headspace aeration $(1 \mathrm{~L} / \mathrm{min})$ by the addition of pure oxygen. The agitation rate of the $100 \%$ air saturation cultures was fixed at 1000 RPM directly after inoculation after which the oxygen tension was controlled by the addition of pure oxygen in the headspace. Samples were taken for optical density measurements and used for nutrient and sOMV 
measurements after sterile filtration $(0.22 \mu \mathrm{m}$ pore-size $)$ and storage at $4{ }^{\circ} \mathrm{C}$. Off-gas composition was analyzed by a Thermo Prima $\delta b$ process mass spectrometer.

\section{Chemostat cultivations}

Continuous cultivations were performed in a similar setup as the batch cultivation setup. The working volume of the 5-liter bioreactor was decreased from 3.0 liter to 2.0 liter to reduce the feed medium required for the experiments. The vessel was equipped with a medium inlet and two outlet pipes, one submerged in the cultivation broth at the height of the stirrer and one directly at the liquid-gas interphase. The latter allowed the control of the working volume to be exactly 2.0 liter at a fixed maximum stirrer speed, independent of foaming. The weight of the bioreactor, the feed medium and the $\mathrm{pH}$ titrant solutions was measured by balances and used for verification of the dilution rate. Samples were taken for optical density measurements and off-gas analysis was similar to the batch cultivation. The bioreactor was controlled with the same control loops as used in the batch cultivations. After 8 hours of growth the feed and the bleed pumps were started to initiate a continuous culture. Steady state of the culture was assumed based on stable bacterial density values and stable carbon dioxide emission for at least 3 dilutions of the bioreactor volume.

\section{Accelerostat and dissolved oxygen changestat cultivation}

An accelerostat was started from a chemostat fermentation in steady state at D $=0.03 \mathrm{~h}^{-}$ 1 , operated as described in the previous section, by increasing the dilution rate linearly with $\mathrm{a}_{\mathrm{D}}=0.0055 \mathrm{~h}^{-2}$. The dilution rate was changed by increasing the medium inflow rate and equally increasing the broth outflow rate. From the culture broth, $50 \mathrm{~mL}$ samples were drawn to purify sOMVs. The samples were centrifuged at $4000 \times g$ for 30 $\min$ at $4{ }^{\circ} \mathrm{C}$ and the sterile filtered supernatants (Nalgene RapidFlow $0.2 \mu \mathrm{m}$ pore-size PES filter unit) were concentrated on $100 \mathrm{kDa}$ cut-off spin filters. The concentrated sOMVs were washed with 3\% sucrose buffered by $10 \mathrm{mM}$ TrisHCl (pH 7.4) to wash out contaminating proteins. Next, the diafiltrated sOMVs were centrifuged at $125.000 \times g$ for $2 \mathrm{~h}$. The sOMV containing pellet was dissolved in $1 \mathrm{~mL} 10 \mathrm{mM}$ TrisHCl (pH 7.4) with $3 \%$ sucrose.

The dissolved oxygen tension changestat was started from a chemostat culture. For this, a continuous culture in steady state with $\mu=0.04 \mathrm{~h}^{-1}$ was obtained as described previously. During this steady state, the oxygen concentration was controlled at $30 \%$ air 
saturation, the starting point for the changestat. From the start of the changestat, the oxygen concentration was increased linearly with aDoT $=1.0 \% / \mathrm{h}$.

Escherichia coli JC8031 (TolRA) was used for the dissolved oxygen tension changestat of E. coli [29]. A shaker flask culture was started by adding $10 \mu \mathrm{L}$ of frozen glycerol stock ($80^{\circ} \mathrm{C}$ ) to $100 \mathrm{~mL} \mathrm{LB}$ medium (Large Capsules: tryptone $10 \mathrm{~g} / \mathrm{L}$, yeast extract $5 \mathrm{~g} / \mathrm{L}, \mathrm{NaCl}$ $10 \mathrm{~g} / \mathrm{L}, \mathrm{MP}$ Biomedicals) and incubating the shaker flask at $37^{\circ} \mathrm{C}$ for 16 hours.

Bioreactor cultivations were performed on LB medium without antifoam with a maximum stirrer speed of $600 \mathrm{RPM}$ at $37^{\circ} \mathrm{C}$.

\section{Quantification of sOMVs}

Culture samples were sterile filtered $(0.22 \mu \mathrm{m}$ pore-size $)$ before the sOMV were measured. sOMV concentration was measured with a phospholipid specific probe FM 464 (SynaptoRed C2, Biotium) by mixing $50 \mu \mathrm{L}$ of 2 to 50 -fold diluted samples with $50 \mu \mathrm{L}$ of dye solution (0.05 mM FM 4-64). Fluorescence was measured directly after mixing this solution using a plate fluorometer (Synergy MX, Biotek ex480, em650). The concentration of SOMV in the culture supernatants was calculated from a calibration curve which was based on the responses of the standards (sOMV corresponding with 0 $-2.5 \mathrm{mg} / \mathrm{L}$ total protein). In the changestat experiments, nanoparticle tracking analysis [30] was used for sOMV quantification. Static measurements (10 captures of 30seconds) were made on a NanoSight NS500 with $488 \mathrm{~nm}$ laser module and sCMOS camera, that was calibrated with the concentration upgrade [31]. Temperature was controlled at $25^{\circ} \mathrm{C}$ and captures were analyzed with the NTA 3.2 software build 3.2.16. Automated flow measurements were made as described previously [32].

OMV size was assessed by dynamic light scattering in a Zetasizer Nano-ZS with Zetasizer 7.11 software (Malvern Instruments). Measurements were performed using a SOP that takes three measurements in backscatter mode, with auto measurement duration and "seek for optimal position" as positioning setting. The sample was assumed to be protein with a refractive index of 1.450 and 0.001 absorption, in water as dispersant with a viscosity of $0.8872 \mathrm{cP}$ and refractive index of 1.330 . Data was processed with the normal analysis model. 


\section{SDS-PAGE}

Purified OMVs were assessed for total protein content by the Lowry protein assay using Peterson's modification. OMVs corresponding to $4 \mu \mathrm{g}$ of protein were loaded on a precast polyacrylamide gel (Lonza) to perform SDS-gel electrophoresis. The gel was stained with InstantBlue protein stain (Expedeon). 


\section{Results}

\section{Neisseria meningitidis SOMV release at reduced growth rate}

The increased productivity of OMVs during the stationary phase of a batch cultivation [20] raised the question what the direct influence of the growth rate on the sOMV release was. Here we assessed the influence of growth rate on sOMV release in three chemostat cultures in steady state at different dilution rates, and in an accelerostat, by slowly increasing the dilution rate of a chemostat culture of $N$. meningitidis. The slow change in dilution rate $\left(a_{D}\right)$ should keep the culture in steady state in this approach [33]. In this accelerostat an acceleration rate $\left(a_{D}\right)$ of $0.0055 \mathrm{~h}^{-2}$ was used (Figure 1B). The carbon dioxide evolution rate (CER) and the optical density increased simultaneously with the dilution rate (Figure 1A). In the accelerostat, OMVs were produced during the whole culture and were also similar in size and protein composition throughout the culture (Figure 1C-D). The specific sOMV production was constant throughout the culture with the growth rate ranging from $0.03 \mathrm{~h}^{-1}$ to $0.18 \mathrm{~h}^{-1}$ (Figure 1B). Chemostat cultures at three different growth rates showed comparable productivity to the accelerostat at a growth rate of $0.18 \mathrm{~h}^{-1}$, while the two lower growth rates show a minor increase in OMV productivity. From these results, we conclude that reducing the growth rate from $0.18 \mathrm{~h}^{-1}$ to $0.03 \mathrm{~h}^{-1}$ is not an important trigger for sOMV release.
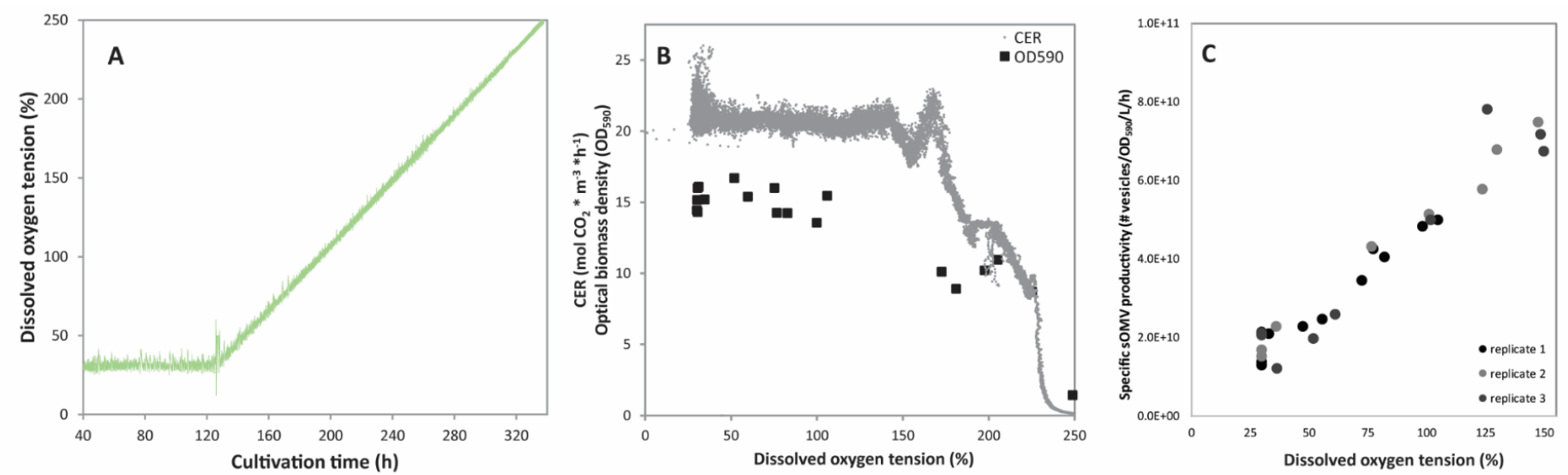

Figure 1. Influence of the growth rate on $\mathrm{OMV}$ release in a $\boldsymbol{N}$. meningitidis accelerostat. Graph A shows the optical density (black squares) and the carbon dioxide evolution rate of the accelerostat culture (grey line). Graph B shows the increase of the dilution rate (black line, $a_{D}$ of $0.0055 \mathrm{~h}^{-2}$ ), the actual measured dilution rate (diamonds). Graph C shows the resulting specific OMV productivity (mg of total protein (TP) per liter culture of $\mathrm{OD}_{590}=1$ per hour) at different dilution rates for the accelerostat (solid circles) and chemostats (open circles). Vesicles were purified from the accelerostat at different dilution rates and the size of the purified OMVs is shown in Graph D. Error bars represent the standard deviation of the measurement. The protein composition of the OMVs is analysed by SDS-PAGE (Graph E). Lane 1 contains a molecular weight marker and lane 2-8 contain sOMVs purified at increasing dilution rates. 
steady state culture (Figure 2A). N. meningitidis is capable of growth up to $150 \%$ air saturation without significant impact on the carbon dioxide evolution rate (Figure 2B). The release of sOMVs is linearly linked to the concentration of oxygen in the culture broth (Figure 2C). sOMV production can be increased by a factor 4 at high oxygen concentration, while preserving growth of the bacteria. Between dissolved oxygen concentrations of $150 \%$ and $220 \%$ air saturation, bacterial growth is affected, illustrated by the declined carbon dioxide production rate and lower biomass concentration (Figure 2B). Production of OMVs at these levels is not preferred.
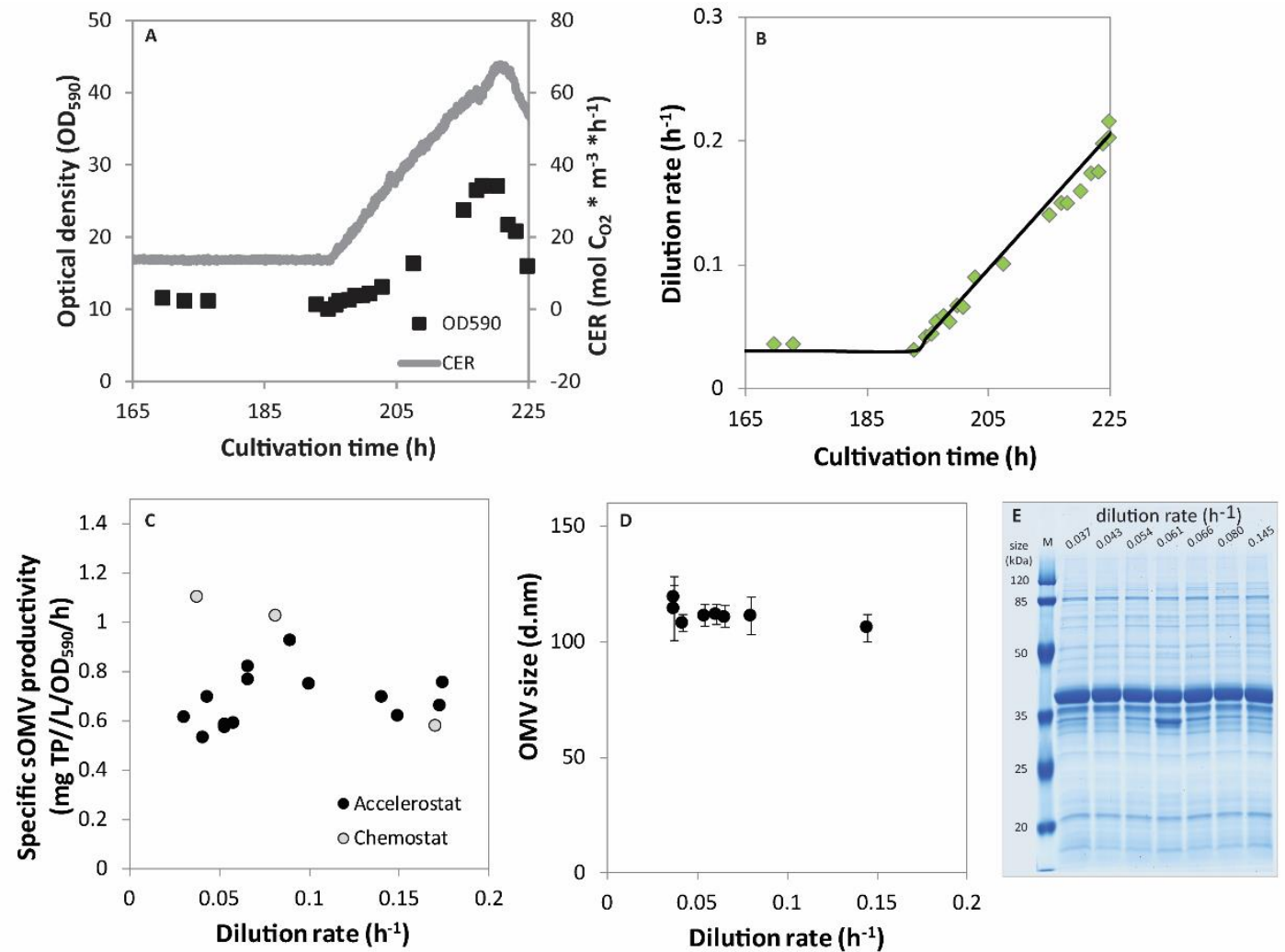

Figure 2. The influence of increased dissolved oxygen tension on growth and OMV productivity.

Graph A shows the control of the dissolved oxygen concentration in the changestat, where the dissolved oxygen tension was increased by $1 \%$ per hour. The effect of the elevated oxygen concentration on the growth is shown in Graph B. The carbon dioxide evolution rate (grey) and bacterial biomass density (black squares) is similar for oxygen concentrations up to $150 \%$ air saturation. Graph C shows the specific productivity of sOMVs as a function of DO for three replicate cultures. The results for replicate 1 as shown in graph A and B are representative for the two other replicates. 
Next, the changestat was repeated until a dissolved oxygen concentration of $150 \%$ after which the setpoint was maintained constant at this value. The culture showed a steady state productivity at a similar level as at the corresponding oxygen concentration during the changestat (Figure 3A), confirming that the accelerating factor of the changestat was sufficiently low to keep the culture in steady state. Last, a third changestat culture was done where after reaching a dissolved oxygen concentration of $150 \%$ the setpoint was returned to the starting value of $30 \%$. The specific productivity returned to the level at the start of the changestats indicating that the changestat culture did not induce changes to the bacteria (Figure 3B). Furthermore, the increased oxygen concentrations also showed to trigger sOMV release in an E. coli dissolved oxygen changestat (Figure S1). In summary, it is shown that increased oxygen tension triggers OMV formation.
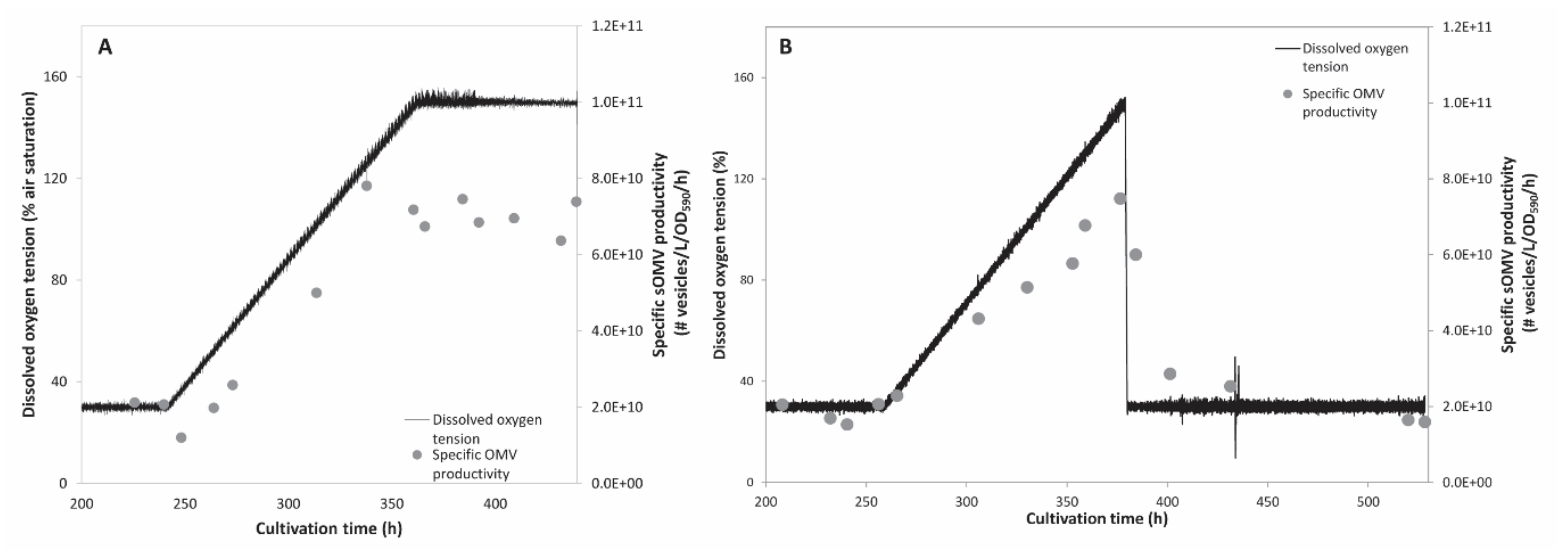

Figure 3. Verification of the dissolved oxygen changestat cultures. One replicate of the $N$. meningitidis dissolved oxygen changestat was maintained at $150 \%$ air saturation upon reaching this value (A). The specific SOMV productivity remained at $7 \times 10^{10}$ sOMVs per liter culture $0_{590}=1$ per hour, confirming the measurements in the changestats. Another replicate was returned to a steady state at $30 \%$ air saturation (B). During 5 dilutions, wash out of the OMVs produced at increased oxygen concentrations during the changestat was observed, resulting in a steady with similar productivity $\left(1.6 \times 10^{10} \mathrm{sOMVs}\right.$ per liter culture $\mathrm{OD}_{590}=1$ per hour $)$ to the beginning of the changestat $\left(1.7 \times 10^{10}\right.$ sOMVs per liter culture $0 D_{590}=1$ per hour).

\section{Improved productivity of batch cultures at increased oxygen concentrations}

The high dissolved oxygen concentration was applied to a N. meningitidis batch cultivation to assess the feasibility of increased sOMV production. A dissolved oxygen tension of $100 \%$ air saturation was used since this value showed increased OMV release while maintaining similar growth characteristics as at 30\% air saturation in the 
changestat (Figure 2B). Bacteria were grown in chemically defined medium that results in SOMV release from the onset of the stationary phase. The bacterial growth profile was similar for the batch cultures at 30\% and 100\% air saturation, showing the capability of N. meningitidis to deal with higher oxygen concentrations (Figure 4A). The higher oxygen concentration triggered an increased release of vesicles resulting in a three-fold higher productivity at the end of the culture compared to the standard level of $30 \%$ (Figure 4B). The size of OMVs remains constant throughout the culture and is similar between the two oxygen concentrations (Figure 4C). High dissolved oxygen levels are therefore a convenient method for increasing sOMV production in batch cultures.
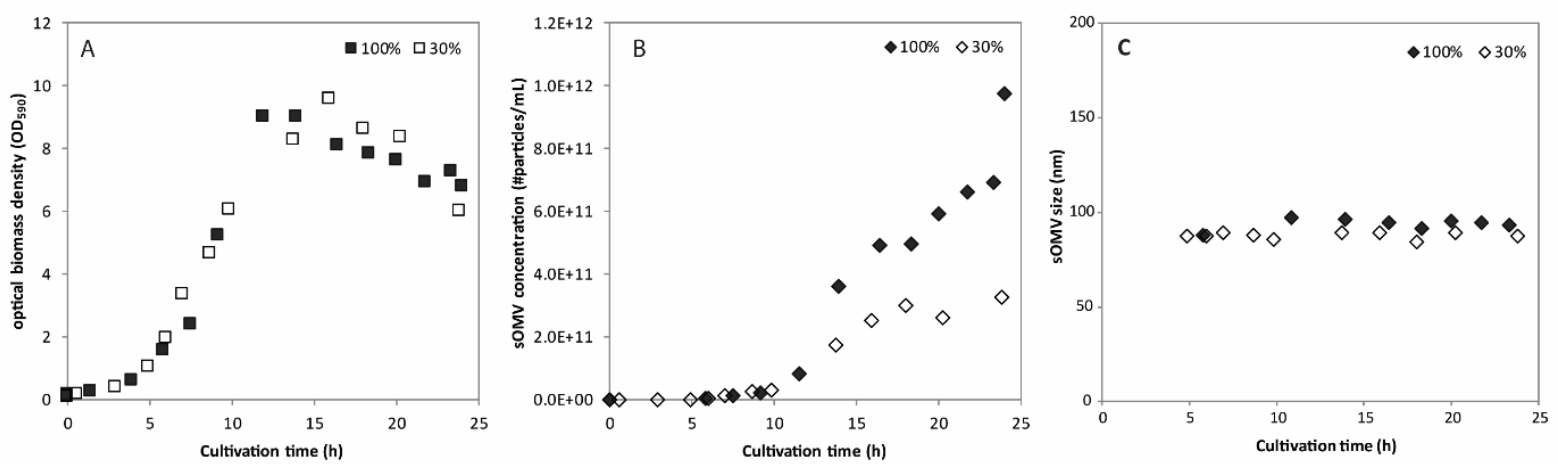

Figure 4. High dissolved oxygen tension induces OMV release in $\boldsymbol{N}$. meningitidis batch cultures. Growth curves of N. meningitidis batch cultures controlled at 30\% (open symbols) and 100\% air saturation (solid symbols) show similar growth (Graph A). The increased oxygen concentration showed to induce a higher level of vesicle release (Graph B). Graphs are the overlay of two replicate cultures to practically allow for sufficient data points covering $24 \mathrm{~h}$. The first replicate consists of data points at $0 \mathrm{~h}$ to $12 \mathrm{~h}$ cultivation and at $24 \mathrm{~h}$ cultivation, and the second replicate at $0 \mathrm{~h}$ and $15 \mathrm{~h}$ to $22 \mathrm{~h}$. Graph $\mathrm{C}$ shows the mode size of sOMV particles as measured by NTA in the supernatants from a N. meningitidis batch cultivation at two dissolved oxygen concentrations (30\% air saturation and $100 \%$ air saturation).

\section{Discussion}

In this study, we investigated reduced growth rate and oxidative stress as triggers to induce sOMV formation in N. meningitidis. In the accelerostat experiment, the growth rate increases linearly with the CER up to a growth rate of $0.18 \mathrm{~h}^{-1}$. At higher dilution rates, a reduction in CER was observed and the experiment was stopped. The maximum specific growth rate of $N$. meningitidis on this medium is $0.5 \mathrm{~h}^{-1}$ [14] and wash-out is thus not expected at this dilution rate. A change in limiting substrate could explain the 
results at growth rates over $0.18 \mathrm{~h}^{-1}$. All chemostats showed depletion of the carbon sources glucose and glutamate and the cultures were likely carbon limited. A lowered bacterial density was observed at reduced bacterial growth rates, that could be explained by the increased energy requirement for maintenance. The biomass yield on substrate $\left(\mathrm{Y}_{\mathrm{xs}}\right)$ and the maintenance coefficient $\left(\mathrm{m}_{\mathrm{s}}\right)$ were 0.43 gbiomass. $_{\text {gglucose }}{ }^{-1}$ and 0.08 gglucose. gbiomass $^{-1} \cdot \mathrm{h}^{-1}$, calculated using the maintenance model of Pirt [34]. These values were in line to the values of aerobic glucose limited chemostat cultures reported by Baart et al. (0.44 gbiomass.gglucose ${ }^{-1}$ and 0.04 gglucose.gbiomass $^{-1} \cdot \mathrm{h}^{-1}$ for resp. $\mathrm{Y}_{\mathrm{xs}}$ and $\mathrm{m}_{\mathrm{s}}$ ) [28]. For the chemostats there seems to be an increase in specific productivity when the growth rate is lowered, where the point at the lowest growth rate significantly deviates from the accelerostat measurement. Since the lowest growth rate is the starting point of the accelerostat, which is certainly in steady state, this difference is not due to a too high acceleration rate in the accelerostat. Together, this data shows that reducing the growth rate from $0.18 \mathrm{~h}^{-1}$ to $0.03 \mathrm{~h}^{-1}$ does not have a large effect on OMV productivity, although due to the contrasting results it is not clear if a minor increase in productivity is associated with lowered growth rates.

The effect of oxidative stress was assessed by changestat cultures with increasing dissolved oxygen tensions. $N$. meningitidis showed to be capable of handling dissolved oxygen concentrations of up to $220 \%$ air saturation. OMV productivity was increased four-fold in a changestat culture at oxygen concentrations elevated to $150 \%$ air saturation and three-fold in a batch culture controlled at $100 \%$ air saturation in comparison to cultures at 30\% air saturation. Applying increased dissolved oxygen tension on E. coli resulted in a similar increase in SOMV release. The production of sOMVs by oxidative stress could be triggered in the bioreactor by controlling the oxygen concentration in the culture broth, although the exact route of OMV induction remains to be elucidated. Oxidative stress triggers SOMV release on top of the known genetic mutations that increase OMV formation $[14,19,35]$. These mutations reduce the linkage between the outer membrane and the peptidoglycan layer. Here we show the effect of oxidative stress on a rmpM knockout strain of $N$. meningitidis and on a Tol-Pal mutant strain of E. coli. Oxidative stress may be a general mechanism to induce sOMV release. Applying increased oxygen concentrations on a batch culture showed enhanced release of OMVs of similar size to OMVs produced in a batch culture with sulfur depletion alone, which is an indication that oxidative stress is the underlying trigger in OMV release 
triggered by sulfur depletion. Moreover, Sabra et al. showed by electron micrographs that Pseudomonas aeruginosa seldom forms membrane vesicles under anoxic conditions ( $\sim 0 \%$ of air saturation), while under extreme oxidative stress conditions (350\% of air saturation) membrane vesicles were observed [36]. Biologically Neisseria spp. encounter oxidative stress upon oxidative bursts of phagocytes [37, 38]. Lappann et al. showed that OMVs of $N$. meningitidis serve as a decoy for the bacteria to circumvent binding of the bacteria to neutrophil extracellular traps (NETs) by binding of OMVs to the NETs [39]. The response of forming OMVs by the bacterium could thus enhance bacterial survival by avoiding phagocytosis and NET-mediated killing. During infection, sOMV release probably contributes to disease progression and the severity of fulminant meningococcal sepsis $[40,41]$. The biological role of OMVs in the interaction with phagocytes should gain more interest. Another explanation of OMV release under oxidative stress conditions would be that the OMVs alleviates stress of the bacterium. This method of stress release could be in the form of eliminating misfolded and unfolded proteins, as shown for Pseudomonas aeruginosa[42]. For E. coli, OMV formation has been shown advantageous to bacterial survival as response to periplasmic protein accumulation, and periplasmic peptidoglycan and LPS fragment accumulation was found to be associated with increased OMV release $[43,44]$. Increased OMV release as response to increased oxidative stress could be advantageous to the bacterium in a similar manner.

During exponential growth in the batch culture, only minor OMV production is observed and high dissolved oxygen tension does not induce OMV release in this phase. A probable explanation is that $N$. meningitidis can handle the increased oxygen concentrations by their metabolism during unlimited growth. Production of oxidative stress is a characteristic of aerobic bacterial growth as components of the respiratory chain are oxidized [45]. Neisseria spp. are oxidase positive pathogens containing a mitochondrial like respiratory chain [46] and typically show high levels of respiration [47]. The $N$. meningitidis genome encodes multiple small c-type cytochromes and a single terminal cytochrome oxidase of the cbb3 type [48-51]. Li et al. hypothesized that the high respiratory capacity of Neisseria spp. and the excess capacity for oxygen reduction acts as defense against endogenous reactive oxygen species (ROS) [50]. SodA and MntC are the major effectors involved in the Neisseria spp. oxidative stress response $[52,53]$. Upon cysteine depletion in batch cultures, high oxygen concentrations enhance 
the production of sOMVs. This increased release may be caused by a reduction in capacity of handling oxidative stress by the oxidative stress defense mechanisms due to cysteine limitation.

Our initial results show that OMV size was not affected although oxidative stress can cause damage to bacteria. In general, increased oxygen concentrations could affect bacterial growth and the production of biological compounds [54], as was observed in the changestat culture at concentrations over $150 \%$ air saturation. Neisseria spp. are adapted to ROS production, since reactive oxygen species accumulate as byproducts of the aerobic respiration $[55,56]$. They thus contain several methods to handle ROS $[49$, 52]. The changestat experiments showed that increased oxygen concentrations can be controlled such that growth remains possible. Future work should ensure the quality of OMVs produced under oxidative stress remains consistent. Promising applications such as the additions and stabilization of enzymes on OMVs [57-59], the study of proteins in their native membrane environment [60], or the delivery of drugs packed in OMVs to specific cells [61] could also benefit from this production method.

\section{Conclusion}

In summary, this study shows that the dissolved oxygen tension of $N$. meningitidis cultivations could be used to stimulate OMV release by the introduction of oxidative stress. Increasing the dissolved oxygen concentration of batch cultures from $30 \%$ to $150 \%$ resulted in a factor 4 increased specific productivity. The dissolved oxygen tension is a well-controlled process parameter to induce outer membrane vesicle formation. With this approach, OMV production can be improved reducing the production costs of OMV-based vaccines and facilitating the use of OMVs for other applications 


\section{List of abbreviations}

OMV Outer membrane vesicle

sOMV Spontaneously released OMV

eOMV Extracted OMV

dOMV Detergent extracted OMV

LPS Lipopolysaccharides

D Dilution rate

$a_{D} \quad$ Acceleration rate of the dilution rate

$a_{D O T}$ Acceleration rate of the dissolved oxygen tension

CER Carbon dioxide evolution rate

OUR Oxygen uptake rate

RQ Respiratory quotient

DO Dissolved oxygen

ROS Reactive oxygen species

TP Total protein

NTA Nanoparticle tracking analysis

NET Neutrophil extracellular trap

$\mathrm{Y}_{\mathrm{px}} \quad$ OMV yield per biomass

$\mathrm{Y}_{\mathrm{xs}} \quad$ Biomass yield on substrate

$\mathrm{m}_{\mathrm{s}} \quad$ Maintenance coefficient

OD590 Optical density at 590nm

\section{Acknowledgements}

The authors thank Leo van der Pol, Peter van der Ley, and Sven van der Kooi for constructive discussions. E. coli strain JC8031 was a kind gift of Roland Lloubes, supplied by Wouter de Jong and Joen Luirink. This work has been funded by the Ministry for Health, Welfare and Sports (The Netherlands).

\section{References}

1. Kulp, A. and M.J. Kuehn, Biological functions and biogenesis of secreted bacterial outer membrane vesicles. Annu Rev Microbiol, 2010. 64: p. 163-84.

2. Schwechheimer, C. and M.J. Kuehn, Outer-membrane vesicles from Gram-negative bacteria: biogenesis and functions. Nat Rev Microbiol, 2015. 13(10): p. 605-19.

3. Dorward, D.W. and C.F. Garon, DNA Is Packaged within Membrane-Derived Vesicles of Gram-Negative but Not Gram-Positive Bacteria. Appl Environ Microbiol, 1990. 56(6): p. 1960-2.

4. Rivera, J., et al., Bacillus anthracis produces membrane-derived vesicles containing biologically active toxins. Proc Natl Acad Sci U S A, 2010. 107(44): p. 19002-7. 
5. Ellen, A.F., et al., Proteomic analysis of secreted membrane vesicles of archaeal Sulfolobus species reveals the presence of endosome sorting complex components. Extremophiles, 2009. 13(1): p. 67-79.

6. Holst, J., et al., Properties and clinical performance of vaccines containing outer membrane vesicles from Neisseria meningitidis. Vaccine, 2009. 27 Suppl 2: p. B3-12.

7. Gorringe, A.R. and R. Pajon, Bexsero: a multicomponent vaccine for prevention of meningococcal disease. Hum Vaccin Immunother, 2012. 8(2): p. 174-83.

8. Fredriksen, J.H., et al., Production, characterization and control of MenB-vaccine "Folkehelsa": an outer membrane vesicle vaccine against group B meningococcal disease. NIPH Ann, 1991. 14(2): p. 67-79; discussion 79-80.

9. Zollinger, W.D., et al., Complex of meningococcal group B polysaccharide and type 2 outer membrane protein immunogenic in man. J Clin Invest, 1979. 63(5): p. 836-48.

10. Lappann, M., et al., Comparative proteome analysis of spontaneous outer membrane vesicles and purified outer membranes of Neisseria meningitidis. J Bacteriol, 2013. 195(19): p. 4425-35.

11. van de Waterbeemd, B., et al., Quantitative proteomics reveals distinct differences in the protein content of outer membrane vesicle vaccines. J Proteome Res, 2013. 12(4): p. 1898-908.

12. van der Ley, P., et al., Modification of lipid A biosynthesis in Neisseria meningitidis lpxL mutants: influence on lipopolysaccharide structure, toxicity, and adjuvant activity. Infect Immun, 2001. 69(10): p. 5981-90.

13. Zariri, A. and P. van der Ley, Biosynthetically engineered lipopolysaccharide as vaccine adjuvant. Expert Rev Vaccines, 2015. 14(6): p. 861-76.

14. van de Waterbeemd, B., et al., Improved OMV vaccine against Neisseria meningitidis using genetically engineered strains and a detergent-free purification process. Vaccine, 2010. 28(30): p. 4810-6.

15. Schwechheimer, C., C.J. Sullivan, and M.J. Kuehn, Envelope control of outer membrane vesicle production in Gram-negative bacteria. Biochemistry, 2013. 52(18): p. 3031-40.

16. Pathirana, R.D. and M. Kaparakis-Liaskos, Bacterial membrane vesicles: Biogenesis, immune regulation and pathogenesis. Cell Microbiol, 2016. 18(11): p. 1518-1524.

17. Haurat, M.F., W. Elhenawy, and M.F. Feldman, Prokaryotic membrane vesicles: new insights on biogenesis and biological roles. Biol Chem, 2015. 396(2): p. 95-109.

18. Roier, S., et al., A novel mechanism for the biogenesis of outer membrane vesicles in Gram-negative bacteria. Nat Commun, 2016. 7: p. 10515.

19. Bernadac, A., et al., Escherichia coli tol-pal mutants form outer membrane vesicles. J Bacteriol, 1998. 180(18): p. 4872-8.

20. van de Waterbeemd, B., et al., Cysteine depletion causes oxidative stress and triggers outer membrane vesicle release by Neisseria meningitidis; implications for vaccine development. PLoS One, 2013. 8(1): p. e54314.

21. Haugaard, N., Cellular mechanisms of oxygen toxicity. Physiol Rev, 1968. 48(2): p. 311-73.

22. Hewitt, C.J., et al., Studies related to the scale-up of high-cell-density E. coli fed-batch fermentations using multiparameter flow cytometry: effect of a changing microenvironment with respect to glucose and dissolved oxygen concentration. Biotechnol Bioeng, 2000. 70(4): p. 381-90.

23. Claassen, I., et al., Production, characterization and control of a Neisseria meningitidis hexavalent class 1 outer membrane protein containing vesicle vaccine. Vaccine, 1996. 14(10): p. 1001-1008.

24. Baart, G.J., et al., Scale-up for bulk production of vaccine against meningococcal disease. Vaccine, 2007. 25(34): p. 6399-408.

25. Holten, E., Serotypes of Neisseria meningitidis isolated from patients in Norway during the first six months of 1978. J Clin Microbiol, 1979. 9(2): p. 186-8.

26. Tommassen, J., et al., Isolation of Neisseria meningitidis mutants deficient in class 1 (porA) and class 3 (porB) outer membrane proteins. Infect Immun, 1990. 58(5): p. 1355-9.

27. Steeghs, L., et al., Neisseria meningitidis expressing lgtB lipopolysaccharide targets DC-SIGN and modulates dendritic cell function. Cell Microbiol, 2006. 8(2): p. 316-25.

28. Baart, G.J., et al., Modeling Neisseria meningitidis metabolism: from genome to metabolic fluxes. Genome Biol, 2007. 8(7): p. R136.

29. Espesset, D., et al., The colicin A pore-forming domain fused to mitochondrial intermembrane space sorting signals can be functionally inserted into the Escherichia coli plasma membrane by a mechanism that bypasses the Tol proteins. Molecular Microbiology, 1994. 13(6): p. 1121-1131.

30. Malloy, A. and B. Carr, NanoParticle Tracking Analysis - The Halo ${ }^{\text {TM }}$ System. Particle \& Particle Systems Characterization, 2006. 23(2): p. 197-204.

31. MalvernInstruments, NanoSight NTA Concentration Measurement Upgrade, in TN150515. 2015.

32. Gerritzen, M.J.H., et al., High throughput nanoparticle tracking analysis for monitoring outer membrane vesicle production. J Extracell Vesicles, 2017. 6(1): p. 1333883. 
33. Paalme, T., et al., The computer-controlled continuous culture of Escherichia coli with smooth change of dilution rate (A-stat). Journal of Microbiological Methods, 1995. 24(2): p. 145-153.

34. Pirt, S.J., Maintenance energy: a general model for energy-limited and energy-sufficient growth. Archives of Microbiology, 1982. 133(4): p. 300-302.

35. Deatherage, B.L., et al., Biogenesis of bacterial membrane vesicles. Mol Microbiol, 2009. 72(6): p. 1395407.

36. Sabra, W., H. Lunsdorf, and A.P. Zeng, Alterations in the formation of lipopolysaccharide and membrane vesicles on the surface of Pseudomonas aeruginosa PA01 under oxygen stress conditions. Microbiology, 2003. 149(Pt 10): p. 2789-95.

37. Moslen, M.T., Reactive oxygen species in normal physiology, cell injury and phagocytosis. Adv Exp Med Biol, 1994. 366: p. 17-27.

38. Ng, V.H., et al., Role of KatG catalase-peroxidase in mycobacterial pathogenesis: countering the phagocyte oxidative burst. Mol Microbiol, 2004. 52(5): p. 1291-302.

39. Lappann, M., et al., In vitro resistance mechanisms of Neisseria meningitidis against neutrophil extracellular traps. Mol Microbiol, 2013. 89(3): p. 433-49.

40. van Deuren, M., et al., Correlation between proinflammatory cytokines and antiinflammatory mediators and the severity of disease in meningococcal infections. J Infect Dis, 1995. 172(2): p. 433-9.

41. Brandtzaeg, P., et al., Plasma endotoxin as a predictor of multiple organ failure and death in systemic meningococcal disease. J Infect Dis, 1989. 159(2): p. 195-204.

42. Baumgarten, T., et al., Membrane Vesicle Formation as a Multiple-Stress Response Mechanism Enhances Pseudomonas putida DOT-T1E Cell Surface Hydrophobicity and Biofilm Formation. Applied and Environmental Microbiology, 2012. 78(17): p. 6217-6224.

43. McBroom, A.J. and M.J. Kuehn, Release of outer membrane vesicles by Gram-negative bacteria is a novel envelope stress response. Molecular Microbiology, 2007. 63(2): p. 545-558.

44. Schwechheimer, C., A. Kulp, and M.J. Kuehn, Modulation of bacterial outer membrane vesicle production by envelope structure and content. BMC Microbiology, 2014. 14: p. 324.

45. Storz, G. and J.A. Imlay, Oxidative stress. Curr Opin Microbiol, 1999. 2(2): p. 188-94.

46. Bøvre, K., Neisseriaceae Prevot 1933, in Bergey's manual of systemic bacteriology, N.R.Krieg\&J.G.Hold, Editor. 1984, Williams and Wilkin: Baltimore, MD.

47. Archibald, F.S. and M.N. Duong, Superoxide dismutase and oxygen toxicity defenses in the genus Neisseria. Infect Immun, 1986. 51(2): p. 631-41.

48. Aspholm, M., et al., Structural alterations in a component of cytochrome c oxidase and molecular evolution of pathogenic Neisseria in humans. PLoS Pathog, 2010. 6(8): p. e1001055.

49. Seib, K.L., et al., Defenses against oxidative stress in Neisseria gonorrhoeae: a system tailored for a challenging environment. Microbiol Mol Biol Rev, 2006. 70(2): p. 344-61.

50. Li, Y., et al., Organization of the electron transfer chain to oxygen in the obligate human pathogen Neisseria gonorrhoeae: roles for cytochromes $c 4$ and c5, but not cytochrome $c 2$, in oxygen reduction. J Bacteriol, 2010. 192(9): p. 2395-406.

51. Deeudom, M., M. Koomey, and J.W. Moir, Roles of c-type cytochromes in respiration in Neisseria meningitidis. Microbiology, 2008. 154(Pt 9): p. 2857-64.

52. Seib, K.L., et al., Defenses against oxidative stress in Neisseria gonorrhoeae and Neisseria meningitidis: distinctive systems for different lifestyles. J Infect Dis, 2004. 190(1): p. 136-47.

53. Tseng, H.J., et al., Accumulation of manganese in Neisseria gonorrhoeae correlates with resistance to oxidative killing by superoxide anion and is independent of superoxide dismutase activity. Mol Microbiol, 2001. 40(5): p. 1175-86.

54. Baez, A. and J. Shiloach, Effect of elevated oxygen concentration on bacteria, yeasts, and cells propagated for production of biological compounds. Microb Cell Fact, 2014. 13(1): p. 181.

55. Korshunov, S. and J.A. Imlay, Detection and quantification of superoxide formed within the periplasm of Escherichia coli. J Bacteriol, 2006. 188(17): p. 6326-34.

56. Imlay, J.A., Cellular defenses against superoxide and hydrogen peroxide. Annu Rev Biochem, 2008. 77: p. 755-76.

57. Alves, N.J., et al., Bacterial Nanobioreactors--Directing Enzyme Packaging into Bacterial Outer Membrane Vesicles. ACS Appl Mater Interfaces, 2015. 7(44): p. 24963-72.

58. Su, F.H., et al., Decorating outer membrane vesicles with organophosphorus hydrolase and cellulose binding domain for organophosphate pesticide degradation. Chemical Engineering Journal, 2017. 308: p. 1-7.

59. Alves, N.J., et al., Environmental Decontamination of a Chemical Warfare Simulant Utilizing a Membrane Vesicle-Encapsulated Phosphotriesterase. ACS Applied Materials \& Interfaces, 2018. 10(18): p. 1571215719. 
60. Thoma, J., et al., Protein-enriched outer membrane vesicles as a native platform for outer membrane protein studies. Communications Biology, 2018. 1(1): p. 23.

61. Gujrati, V., et al., Bioengineered Bacterial Outer Membrane Vesicles as Cell-Specific Drug-Delivery Vehicles for Cancer Therapy. ACS Nano, 2014. 8(2): p. 1525-1537.

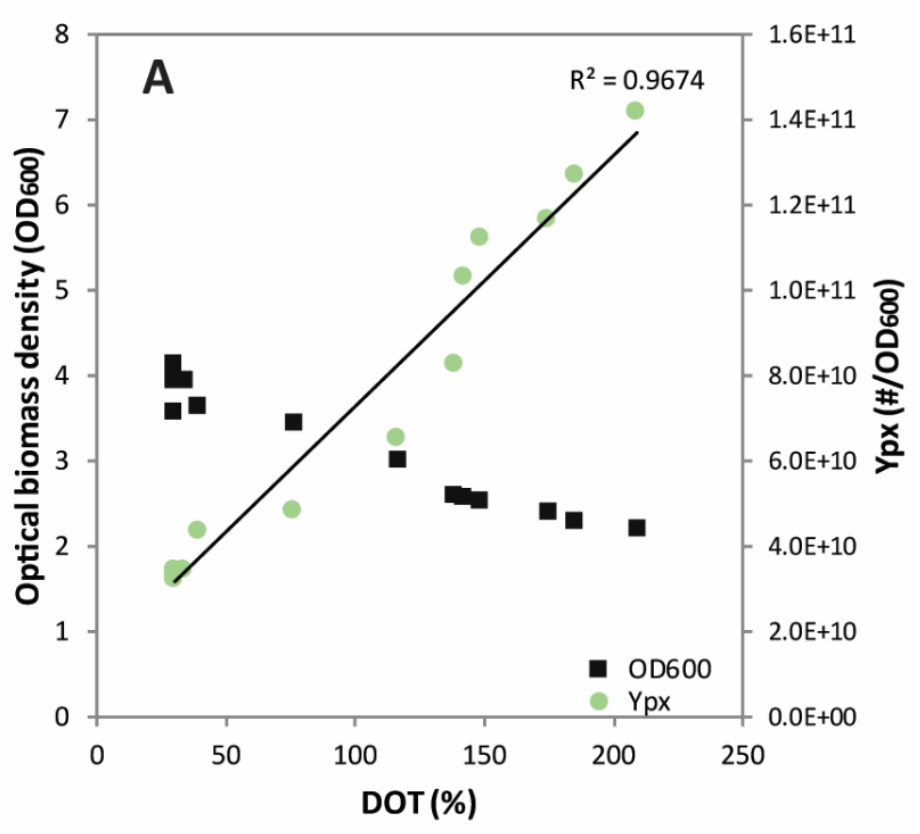

\section{Supplemental Figure 1. Increased} dissolved oxygen tension triggers

OMV release in E. coli. Changestat of $E$. coli (A) shows growth at dissolved oxygen tensions up to $200 \%$ air saturation in a changestat with арот = $1.5 \% / \mathrm{h}$. OMV release is directly related to the increased oxygen concentration. 


\section{Chapter 6}

\section{Spontaneously released Neisseria meningitidis outer membrane vesicles as vaccine platform}

Accepted for publication:

Gerritzen, M.J.H., M.L.M. Salverda, D.E. Martens, R.H. Wijffels and M. Stork (2019). "Spontaneously released Neisseria meningitidis outer membrane vesicles as vaccine platform: Production and purification."

Vaccine (2019) 


\begin{abstract}
Outer membrane vesicles (OMVs) are nanoparticles produced by Gram-negative bacteria that can be used as vaccines. The application of OMVs as vaccine component can be expanded by expressing heterologous antigens on OMVs, creating an OMV-based vaccine platform. This study aims to develop a production process for such OMV-based vaccines and studies the production method based on meningococcal OMVs that express heterologous antigens on their surface. As a proof of concept, the B. burgdorferi antigens OspA and OspC were expressed on Neisseria meningitidis OMVs to create a concept antiLyme disease vaccine. Production of OMVs released in the culture supernatant was induced by high dissolved oxygen concentrations and purification was based on scalable unit operations. A crude recovery of $90 \mathrm{mg}$ OMV protein could be obtained per liter culture. Expressing heterologous antigens on the OMVs did result in minor reduction of bacterial growth, while OMV production remained constant. The antigen expression did not alter the OMV characteristics. This study shows that production of well characterized OMVs containing heterologous antigens is possible with high yields by combining high oxygen concentrations with an optimized purification process. It is concluded that heterologous OMVs show potential as a vaccine platform.
\end{abstract}




\section{Introduction}

Vaccination is one of the most successful inventions as vaccines have saved many lives by preventing infectious diseases. Despite the successes, infectious diseases remain a major source of mortality worldwide. The development of new vaccines is continuously required as new infectious diseases emerge and there is an increasing demand for therapeutic vaccines. There is thus a need for new vaccine production technologies that enable more rapid production of vaccines. Vaccine platforms can provide enhanced safety, productivity and simplicity in vaccine development by thorough initial development of the platform. A vaccine platform for prophylactic vaccines aims at eliciting high and lasting antibody responses against specific antigens, while evoking a standard supportive innate immune response. The design of the vaccine platform should be such that protection against different infectious diseases can be induced by displaying different antigens. Bacterial outer membrane vesicles (OMVs) are suitable candidates for vaccine platforms. OMVs are nanoparticles derived from Gram-negative bacteria that are highly immunogenic while non-infective and non-replicating [1]. However, not all Gram-negative pathogens are suitable OMV vaccine producers because of their high pathogenicity, low growth rate, complex media requirements, lack of genetic accessibility, or low OMV productivity. Adding heterologous antigens to OMVs creates an OMV-based vaccine platform. Bacteria have been engineered so that they produce heterologous antigens in OMVs [2,3]. Alternatively, the antigen can be produced separately and added to the OMV [4-6]. Besides targeting infectious disease targets, OMV-based vaccine platforms can be designed as therapeutic vaccines [7-9]. Gram-negative bacteria produce spontaneously released OMVs (sOMVs) that are secreted in the culture medium. However, the amount of sOMVs produced per amount of biomass is generally low and therefore other methods of OMV production have been developed, like extraction of vesicles from the bacterial cells by detergents (dOMVs) or by EDTA or other detergent-free methods (eOMVs) [10]. Detergent extraction has been used in the production of OMVs since the detergent extraction reduces the lipopolysaccharide (LPS) content of the OMVs resulting in safer to use vaccine compositions. However, with the development of genetically detoxified LPS variants there remains no necessity of detergent extraction to reduce the LPS toxicity of OMVs [11]. 
OMVs from Neisseria meningitidis (Nm) are well studied, used to control outbreaks of serogroup B meningococcal disease, and Nm dOMVs are included in the 4CMenB/Bexsero vaccine [12]. Here we test the expression of heterologous antigens on both Nm eOMV and Nm sOMV. Outer surface protein A (OspA) and Outer surface protein C (OspC) of Borrelia burgdorferi were chosen as model antigens. B. burgdorferi causes Lyme disease and is the most common tick-borne illness in the Northern hemisphere $[13,14]$. Recombinant OspA has been shown safe and effective in randomize controlled trials and has been the basis of novel vaccine concepts [15-17]. These model antigens are surface expressed because we hypothesized that surface exposure of antigens in an OMV vaccine platform is advantageous. However, the induction of antibody responses has also been shown for antigens expressed in the OMV lumen [18-21]. The heterologous antigens are surface expressed by fusion of the proteins to short N-terminal part of the Nm lipoprotein fHbp [22]. Spontaneous OMV production was triggered in batch cultures by cysteine depletion of the culture media [23], which is associated with oxidative stress responses. Additionally, high dissolved oxygen concentrations were used during cultivation to enhance OMV production [24].

This study aims to develop a production platform for Nm OMVs expressing heterologous antigens. First a purification process for sOMVs is designed on the available unit operations of the eOMV purification process. Optimization of the separation of sOMVs from the bacterial cells was required to achieve sufficient yields. Next, the impact of expressing heterologous antigens on the growth of the bacterial culture the quality of OMVs expressing heterologous antigens are assessed. Furthermore, the characteristics of eOMVs and sOMVs are compared.

\section{Methods}

\section{Bacterial strains}

The H44/76 isolate of Neisseria meningitidis serogroup B [25] was used as OMV production host. In this strain the $r m p M$ gene was knocked out to induce vesicle formation [26]. Furthermore, the strain is non-encapsulated by siaD knockout, has

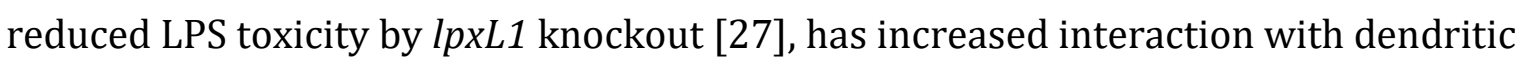
cells by $\lg t B$ knockout [28], and lacks the major outer membrane protein and immunodominant antigen porA [29]. Outer surface protein A (OspA) and Outer surface protein $\mathrm{C}(\mathrm{OspC})$ from Borrelia burgdorferi were expressed in $\mathrm{Nm}$ as heterologous 
antigens. The OspA and OspC genes were codon optimized for the codon usage of $\mathrm{Nm}$ and expressed by placement after a hybride porA-nadA promotor [30]. To ensure surface exposure, OspA and OspC were linked to fHbp (fHbp-OspA, fHbp-OspC) as based on the method described previously [22]. An overview of the strains used in this study is given in Table 1.

Table 3. Overview of bacterial strains used in this study. The heterologous antigens Outer surface protein A (OspA) and Outer surface protein C (OspC) from B. burgdorferi were expressed in $N$. meningitidis ( $\mathrm{Nm}$ ) by insertion of the gene in either the rmpM gene of $\mathrm{Nm}$ or the lpxL1 gene of Nm, disrupting the function of the native Nm gene.

\begin{tabular}{l|ccc} 
Strain & Knockout mutations & $\begin{array}{c}\text { Heterologous antigen } \\
\text { OspA }\end{array}$ & OspC \\
\hline Nm & rmpM, siaD, lgtB, & - & - \\
Nm OspA & $r m p M, \operatorname{sia}$, lgtB, & in $r m p M$ & - \\
Nm OspC & rmpM, siaD, lgtB, & - & in $r m p M$ \\
Nm OspA+OspC & rrmpM, siaD, lgtB, & in lpxL1 & in $r m p M$
\end{tabular}

\section{Batch cultivations}

Pre-cultures were started by adding $10 \mathrm{~mL}$ of frozen working seedlot in to a shaker flask containing $155 \mathrm{~mL}$ chemically defined growth medium [31]. The shaker flask was incubated in a shaker incubator at $35^{\circ} \mathrm{C}, 200 \mathrm{RPM}$ and after growth it was used to inoculate a second shaker flask. At $\mathrm{OD}_{590}=1.5 \pm 0.5$ this was transferred to a bioreactor containing $3 \mathrm{~L}$ medium to start the batch culture. The bioreactor (Pierre Guerin Tryton ${ }^{\mathrm{i}}$ ) controlled the temperature at $35^{\circ} \mathrm{C}, \mathrm{pH}$ at 7.2 . The dissolved oxygen tension was controlled at 30\% air saturation by increased agitation rate (300-1000 RPM) followed by addition of oxygen in the overlay aeration. The growth medium was designed such that cysteine depletion causes growth arrest and triggers sOMV release [23]. sOMV release can be further induced by oxidative stress from high dissolved oxygen concentrations in the culture. These cultures were controlled at $100 \%$ air saturation, further referred to as high dissolved oxygen. Filtered off-gas ( $0.22 \mu \mathrm{m}$ cut-off) was measured by a Thermo Prima $\delta$ b mass spectrometer to monitor oxygen consumption and carbon dioxide production. 


\section{Downstream processing of sOMVs}

The harvested culture broth was cooled to $20^{\circ} \mathrm{C}$ and transferred to a tangential flow microfiltration setup (Spectrum MiniKros pilot or Spectrum KR2i) with a $20 \mathrm{~cm}$ mPES hollow fiber module. The hollow fiber was used according to the manufacturer's instructions (Spectrum Labs). Transmembrane pressure scouting (TMP-scouting) and flow excursion were performed using the harvest of two $3 \mathrm{~L}$ batch cultures grown at $100 \%$ DOT as described above. For the TMP-scouting, $3 \mathrm{~L}$ harvest was cooled to $20^{\circ} \mathrm{C}$ and transferred to a tangential flow filtration (TFF) setup using the Spectrum KR2i and Spectrum $85 \mathrm{~cm}^{2}, 0.65 \mu \mathrm{m}$ pore size hollow fiber module with $0.7 \mathrm{~mm}$ lumen diameter. The permeate flowed back in to the feed. TMP was controlled by the automatic backpressure valve. For the flow excursion, a similar setup was used with a new culture harvest, using a new $85 \mathrm{~cm}^{2} 0.65 \mu \mathrm{m}$ pore size membrane module without backpressure valve. The permeate flow was controlled by the KRJ pump of the KR2i. To produce sOMVs, a $520 \mathrm{~cm}^{2} 0.65 \mu \mathrm{m}$ pore size mPES hollow fiber module was used on a KroFlo MiniKros Pilot TFF system (Spectrum). The cooled harvest was concentrated 6-fold by processing $16.000 \mathrm{~s}^{-1}$ shear rate with a constant flux rate of 15 liter per $\mathrm{m}^{2}$ surface area per hour (LMH). Next, the remaining sOMVs were washed out by 2 volumes diafiltration by constant volume diafiltration performed at the same operating settings, using a 10 mM Tris- $\mathrm{HCl} 3 \%$ sucrose buffer of $\mathrm{pH}$ 7.2. Next, sOMVs were purified starting with the addition of $300 \mathrm{U} / \mathrm{L}$ Benzonase (Merck) to the crude sOMVs to digest DNA. The sOMVs were concentrated using a $790 \mathrm{~cm}^{2}, 100 \mathrm{kDa}$ cut-off mPES membrane, followed by 3 volume diafiltration with buffer (10 mM Tris- $\mathrm{HCl} 3 \%$ sucrose buffer of $\mathrm{pH}$ 7.2). The concentrated OMVs were clarified by dead-end microfiltration ( $1.2 \mu \mathrm{m}-0.5 \mu \mathrm{m}$ cut off $)$ before group separation by size-exclusion chromatography on a Sepharose 6 Fast Flow column (GE Life Sciences). Lastly, sOMVs were sterile filtered using a $0.2 \mu \mathrm{m}$ cut-off dead-end filter (Pall).

\section{Downstream processing of eOMVs}

Nm eOMVs were produced based on the process described by Van de Waterbeemd et al. [32]. In brief, the biomass of the cooled culture harvest was concentrated 5-fold using a $790 \mathrm{~cm}^{2} \mathrm{mPES}$ hollow fiber module with $0.2 \mu \mathrm{m}$ pore size and $0.5 \mathrm{~mm}$ lumen diameter (Spectrum Labs), followed by 2 volumes diafiltration in a buffer suited for OMV extraction (100mM Tris- $\mathrm{HCl}$ pH 8.6). eOMVs were separated from residual bacterial 
cells by 10 volumes diafiltration. Next, purification of eOMVs was identical to the purification of sOMVs described above.

\section{Analytical methods}

OMV samples were quantified by Nanoparticle Tracking Analysis (NTA) [33]. Culture samples were filtered $(0.22 \mu \mathrm{m}$ pore size) before measurement. NTA was performed on a calibrated NanoSight NS500 by capturing 10 captures of 30 seconds at $25^{\circ} \mathrm{C}$. Purified water was used to prepare correct sample dilutions. Automated flow-measurements were obtained as described previously [34]. Captures were analyzed using the NTA 3.2 software build 3.2.16. Total protein content of purified OMV samples was assessed using Lowry's protein assay with Peterson's modification and Bovine Serum Albumin as protein standard. The assay was performed according to manufacturer's protocol (Sigma-Aldrich). LPS content was measured by a modified gas chromatography method $[35,36]$. In brief, the LPS was quantified based on the peak area of C14:0-30H using C12:0-2OH as internal standard [31]. Protein composition of OMVs was assessed by SDS-PAGE by loading OMVs with a total protein content of $4 \mu \mathrm{g}$ on a precast polyacrylamide gel (Lonza) to perform SDS-gel electrophoresis. The gel was stained with InstantBlue protein stain (Expedeon).

For LC-MS/MS analysis, OMVs were denaturated at $100^{\circ} \mathrm{C}$ for $30 \mathrm{~min}$ in potassium phosphate buffer (100 mM, pH 7.8), also containing $100 \mathrm{mM}$ Rapigest (Waters), at a protein concentration of $200 \mu \mathrm{g} / \mathrm{mL}$. Reduction and alkylation of the proteins were performed by subsequent incubations with TCEP $\left(1\right.$ hour at $\left.55^{\circ} \mathrm{C}\right)$ and iodoacetamide (30 minutes at ambient temperature in the dark). Proteins were digested with LysC (0.4 $\mu \mathrm{g}$, Roche) and trypsin ( $1 \mu \mathrm{g}$, Promega) by overnight incubation at $37^{\circ} \mathrm{C}$. Solid-phase extraction was performed to remove excess reagents using 1-mL C18 Sep-pack cartridges (Waters) according to the manufacturer's protocol. The peptide fraction was dried in a vacuum concentrator and reconstituted in $1 \mathrm{~mL}$ of water containing $5 \%(\mathrm{v} / \mathrm{v})$ DMSO and 0.1\% (v/v) formic acid for LC-MS/MS analysis. Nanoscale reversed-phase liquid chromatography was used for peptide separation [37]. Briefly, $10 \mu \mathrm{l}$ of the reconstituted digest sample was loaded on a C18-trapping column $(20 \mathrm{~mm} \mathrm{~L} \mathrm{x} 100 \mu \mathrm{m}$ I.D., made in-house) with solvent $A(0.1 \%(\mathrm{v} / \mathrm{v})$ formic acid in water) in $10 \mathrm{~min}$ at 5 $\mu \mathrm{L} / \mathrm{min}$. The analytes were separated on a C18-analytical column $(27.5 \mathrm{~cm} \mathrm{~L} \mathrm{x} 50 \mu \mathrm{m}$ I.D., made in-house) at a flow rate of $125 \mathrm{~nL} / \mathrm{min}$ with a nonlinear gradient of solvent $\mathrm{B}$ 
$(0.1 \%(\mathrm{v} / \mathrm{v})$ formic acid in acetonitrile): $8 \%$ to $29 \%$ in $140 \mathrm{~min}, 29 \%$ to $60 \%$ in $15 \mathrm{~min}$ and a direct step to $85 \%$ (hold for $10 \mathrm{~min}$ ). The ElectroSpray Ionization interface comprised of a fused silica tapered tip ( $25 \mu \mathrm{m}$ I.D., TipID=3.5 $\mu \mathrm{m}$, made in-house), operated at $2100 \mathrm{~V}$. The peptides were measured in an Orbitrap Fusion Lumos mass spectrometer (Thermo Scientific) utilizing data-dependent scanning: the MS-scan (m/z $300-1500)$ with an orbitrap readout $(120,000$ FWHM). Precursor ions with +2 to +7 charge states (intensity threshold 25,000 counts) were selected for collision-induced dissociation (CID) with an ion trap readout using default settings. Proteome Discoverer 2.1 software (Thermo Scientific) was used for peptide identification and quantification (based on the High-3 method, i.e. the average peak areas of the top 3 peptides for each protein). Identification of peptides was performed against the protein database of $N$. meningitidis H44/76 (NCBI 909420) (2406 entries) and the databases of the fHbp-OspA and fHbp-OspC constructs. Asparagine deamidation and methionine oxidation were set as dynamic modifications while cysteine carbamylation was set as static modification. The data were searched with full trypsin cleavage specificity, allowing 2 missed cleavages. Precursor ion and MS/MS tolerances were set to $5 \mathrm{ppm}$ and $0.4 \mathrm{Da}$, respectively. Peptides were filtered using Perculator (1\% FDR). Reported protein areas were multiplied with their respective molecular masses and used to calculate the percentage of the protein abundance for each individual protein relative to the total sum of all identified proteins. Protein localization was predicted using PSORTb v3.0 [38].

\section{Stability of OspA expression during cultivation}

A bioreactor culture was subcultured in shaker flasks from $\mathrm{OD}_{590}=0.1$ to $\mathrm{OD}_{590}=3.0 \pm 0.5$ for over 20 generations after the end of a batch bioreactor culture. Culture samples from the shaker flasks as well as from the frozen seedlots, the preculture and the bioreactor culture were analyzed for the presence of OspA by Western blot analysis. As primary antibody a polyclonal rabbit anti-OspA (Rockland) was used with goat-antirabbit IgG-AP (Southern BioTech) as secondary antibody. 


\section{Results}

\section{Overall process design for SOMV production}

A production process for Nm sOMVs was designed based on the scalable unit operations of the Nm detergent-free extracted OMV (eOMV) production process (Figure 1). The production of biomass starts from a frozen working cell-bank, by growth in a shaker flask (1.1). After growth, this is subcultured to an inoculum culture (1.2) that is subsequently used to start a bioreactor production culture (1.3). The culture is cooled to reduce bacterial activity (1.4) during OMV purification. The biomass is concentrated (2.0) for eOMV production using tangential flow filtration (TFF). Next, the bacteria are diafiltrated to extraction buffer on the same hollow fiber module (2.1). eOMVs are extracted by incubation with EDTA (2.2) and separated from the residual bacterial biomass by collecting the filtrate (3.0). Next, eOMVs are purified by DNA degradation (4.0) and concentrated on a $100 \mathrm{kDa}$ cut-off hollow fiber module (4.1). The concentrated OMVs are changed to a different buffer (4.2) and clarified (5.1) before separation from soluble proteins by size-exclusion chromatography (6.1). Lastly, the purified eOMVs are filtered to obtain sterile OMVs (7.1). For sOMVs, purification starts by separation of the SOMVs from the bacterial biomass by tangential flow filtration (2.0). Further purification of SOMVs is equivalent to the eOMV process, omitting the extraction process (2.1-3.0). In the following sections the sOMV separation process from the biomass is further discussed, followed by a proof of concept of OMV production of Nm OMVs containing heterologous antigens and analysis of their quality.

\section{Optimizing tangential flow microfiltration-based recovery of OMVs}

Tangential flow microfiltration was used to separate sOMVs from the bacterial cells. A process was tested based on the process of biomass concentration used previously in eOMV production. Where for eOMV production tangential flow microfiltration is used for concentrating the biomass (step 2.0), for sOMV production, the same step is used to remove bacterial cells. A cooled Nm culture was processed by operating an $0.2 \mu \mathrm{m}$ pore size hollow fiber filter in constant transmembrane pressure (TMP) mode. The production of $\mathrm{Nm}$ sOMVs yielded a total of $4 \times 10^{14}$ particles in the bacteria-free OMV fraction, which corresponds to a recovery of $77 \%$ of the $5 \times 10^{14}$ OMVs produced in a $3 \mathrm{~L}$ culture (Figure 2A). The productivity of another culture was improved four-fold to $2.0 \times 10^{15}$ sOMVs in 3L culture broth by increasing the dissolved oxygen concentration to 
$100 \%$ air saturation. Surprisingly, the recovery of sOMVs in step 2.0 (TFF

microfiltration) from this culture was reduced to 1.0\% (Figure 2A).

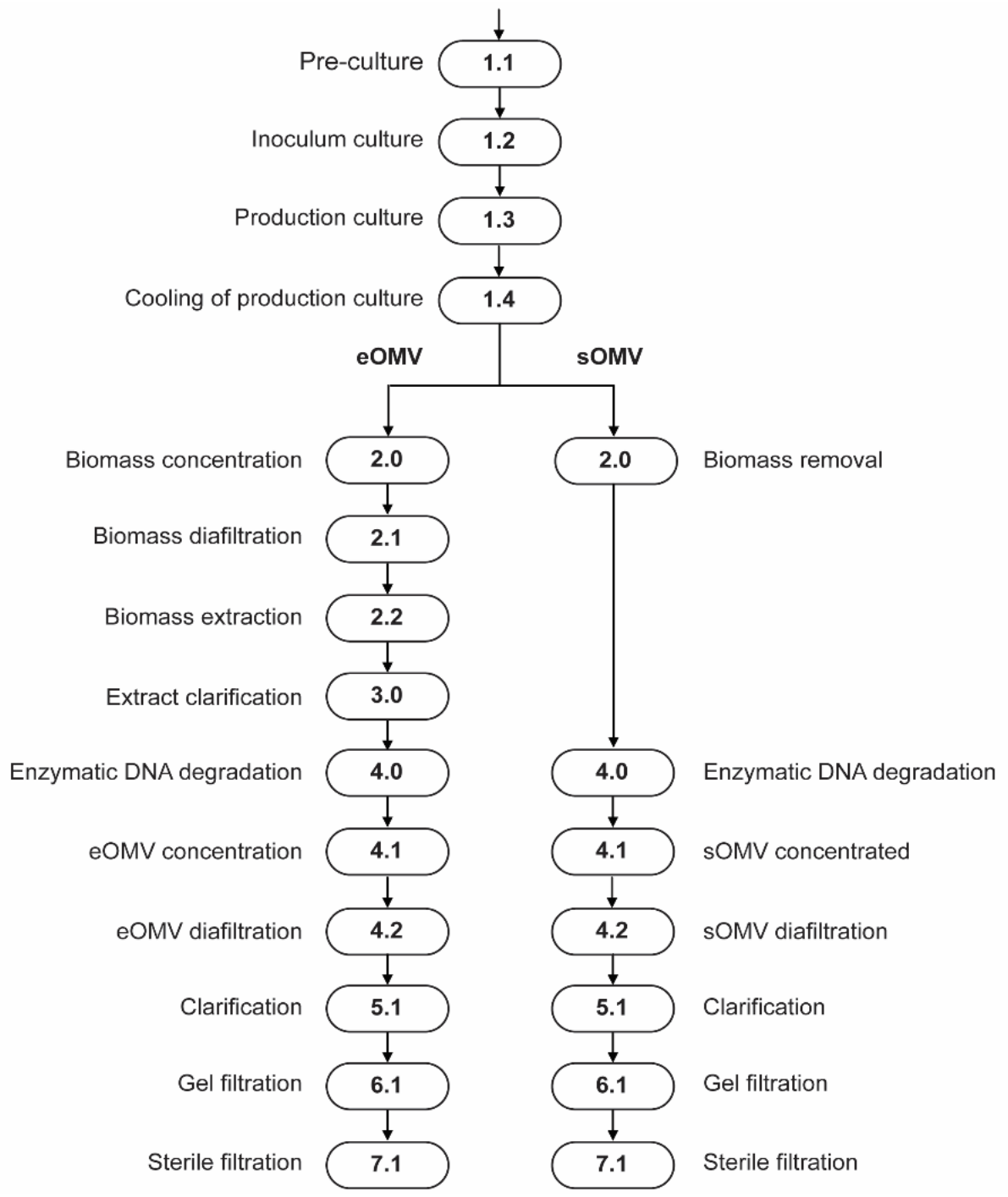

Figure 1. Schematic production process of eOMVs and sOMVs. Production of both $\mathrm{Nm}$ eOMV as Nm sOMV starts by batch cultivation of $N$. meningitidis on chemically defined growth medium. After bacterial growth, sOMVs can be purified from the culture supernatant directly, while the production of eOMVs requires an extraction process of the concentrated biomass under specific conditions. Further down-stream processing of both types of OMVs consists of the same process steps: DNA removal, vesicle concentration, buffer exchange, removal of residual proteins, and sterile filtration. The unit operations are explained in the main text.

OMV induction by high dissolved oxygen concentration showed severely reduced recovery of sOMVs using the $0.2 \mu \mathrm{m}$ pore size hollow fiber filter. Since the size of the OMVs $(100 \mathrm{~nm})$ is close to the cutoff of the $0.2 \mu \mathrm{m}$ pore size membrane, transmission can be affected by small increases in OMV size or slight fouling of the filter pores. Since we found previously that the size of OMVs was not affected by the increased oxygen levels, we hypothesized that the mild stress may have caused a decrease in pore size due to DNA or other cell debris. We assessed the performance of a hollow fiber membrane filter with increased pore size to restore the recovery of sOMVs. A pore size of $0.65 \mu \mathrm{m}$ resulted in complete retention of bacteria, which are $0.6-1.0 \mu \mathrm{m}$ in diameter, and OMV recovery increased to 29\% (Figure 2B). Additionally, it was tested whether DNA degradation by DNAse before filtration would improve the transmission, but the transmission was similar (31\%) to processing without DNA degradation (Figure 2B). 

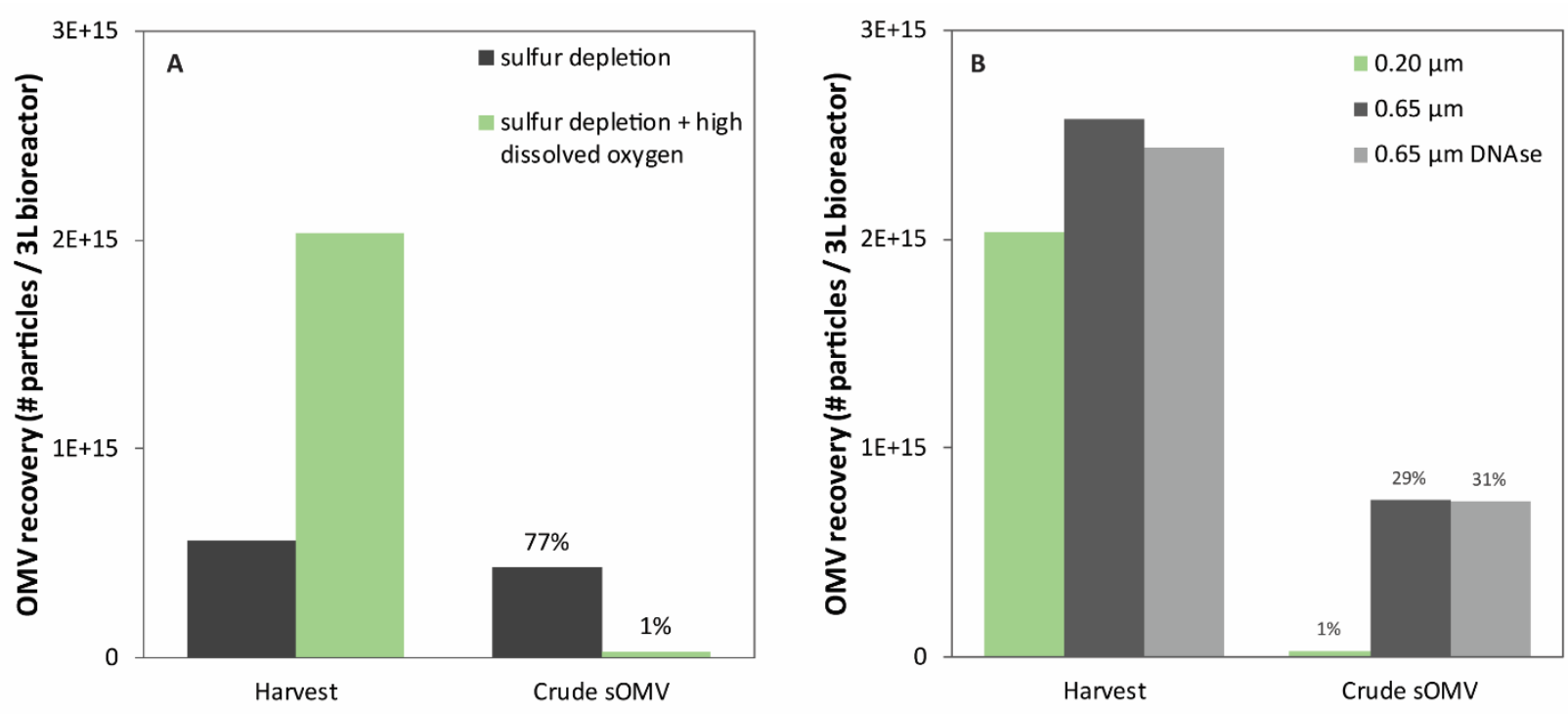

Figure 2. N. meningitidis biomass removal and sOMV recovery by tangential flow filtration.

Recovery of sOMVs by tangential flow filtration at constant TMP from a bioreactor culture (harvest) (A). Nm sOMVs produced in a sulfur-source (cysteine) depleted culture (black) showed $77 \%$ recovery, while a sulfur-source depleted culture combined with a high dissolved oxygen concentration (green) showed $1 \%$ recovery. Recovery of sOMVs from a culture with high oxygen concentration by tangential flow filtration at constant TMP is optimized using an increased pore size membrane and DNAse treatment (B)

Further analysis of the filtration performance showed that initial transmission of OMVs over the $0.65 \mu \mathrm{m}$ pore size membrane was high, but reduced quickly, indicating fouling of the filter membrane. To explore the possibilities of improving the recovery of sOMVs induced by the high dissolved oxygen concentration, TMP scouting was performed. By measuring the flux at different transmembrane pressures, an optimum flux can in princicple be found at increased shear rates and increased transmembrane pressures (Figure 3A). Interestingly, we observed that the passage of OMVs through the filter was highly reduced at increasing transmembrane pressures (Figure 3B), while the transmission of total protein remained unaffected for all samples (data not shown). Especially at a moderate shear rate of $5000 \mathrm{~s}^{-1}$, the cross flow along the membrane was apparently not strong enough to clean the surface. If only a minor amount of fouling occurs, it reduces the possibility of the relatively large OMVs to pass the pores of the membrane. Operating the membrane at higher TMPs will thus cause a reduced recovery of OMVs. 

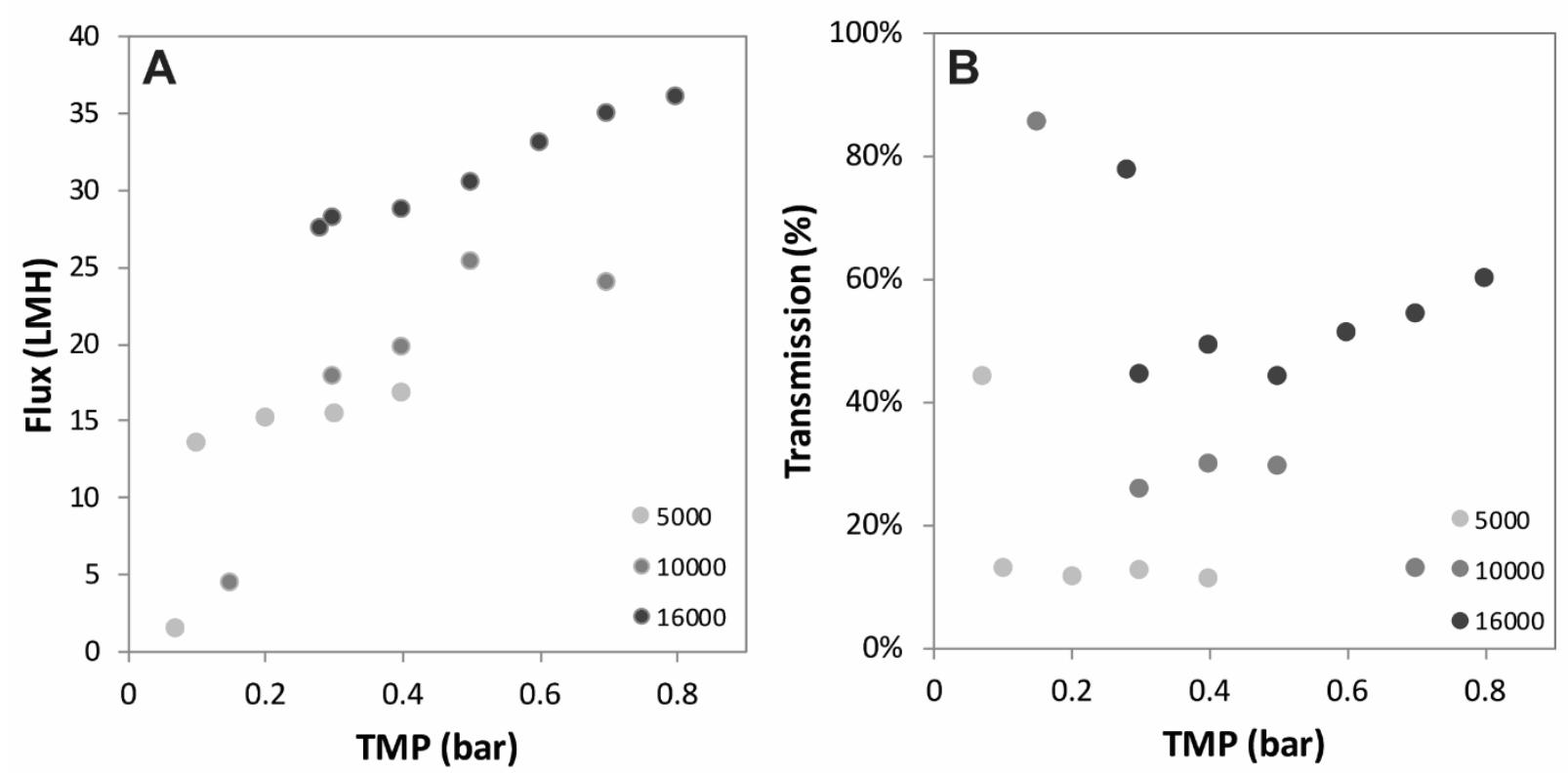

Figure 3. TMP scouting of the TFF of a dissolved oxygen induced sOMV harvest. Filter performance at varying shear rates and increasing transmembrane pressures using a $N$. meningitidis high dissolved oxygen induced sOMV harvest. The resulting permeate flux in liter per $\mathrm{m}^{2}$ surface area per hour (LMH) is shown at increasing transmembrane pressures (A). The transmission of sOMVs at increasing transmembrane pressures is given for crossflow rates at 5,000 s-1 shear, $10,000 \mathrm{~s}^{-1}$ shear, and $16,000 \mathrm{~s}^{-1}$ shear (B).

Minimization of membrane fouling by this highly fouling culture harvest can be achieved by operating with a constant permeate flow, instead of at constant TMP. This constant flux method ensures the lowest fouling conditions since the flow through the pores of the membranes is limited by the permeate pump, while the cleaning action of the flow along the membrane is maintained. To determine the operating conditions for this mode, the critical flux was determined at three different shear rates (Figure 4). The shear rate of 5,000 s-1 showed the lowest critical flux (10 LMH), while the shear rates $10,000 \mathrm{~s}^{-1}$ and $16,000 \mathrm{~s}^{-1}$ showed higher critical fluxes (22 LMH). Transmission of the OMVs through the membrane was constant up to the critical flux. For the higher shear rates transmission was constant at 70\%. Processing the culture harvest at high shear rates and a flux below the critical flux should allow the processing of the entire harvest volume with $70 \%$ transmission. By processing $5 / 6^{\text {th }}$ of the volume, followed by 2 volumes diafiltration, theoretically $93 \%$ of OMVs could be recovered. 


\section{Production of meningococcal sOMVs containing heterologous antigens}

The process with optimized microfiltration (step 2.0) was used to produce Nm sOMVs, and $\mathrm{Nm}$ sOMVs containing heterologous antigens. Heterologous antigens were added to sOMVs by genetical linkage to an outer membrane linking protein of $N$. meningitidis to ensure surface exposure [22]. Fusion constructs of fHbp to Outer Surface Protein A (OspA) and Outer Surface Protein C (OspC) of Borrelia burgdorferi were expressed individually in $\mathrm{Nm}(\mathrm{Nm}$ OspA, $\mathrm{Nm}$ OspC) as well as combined ( $\mathrm{Nm}$ OspA + OspC). The stability of the heterologous expression of antigens was analyzed for the OspA
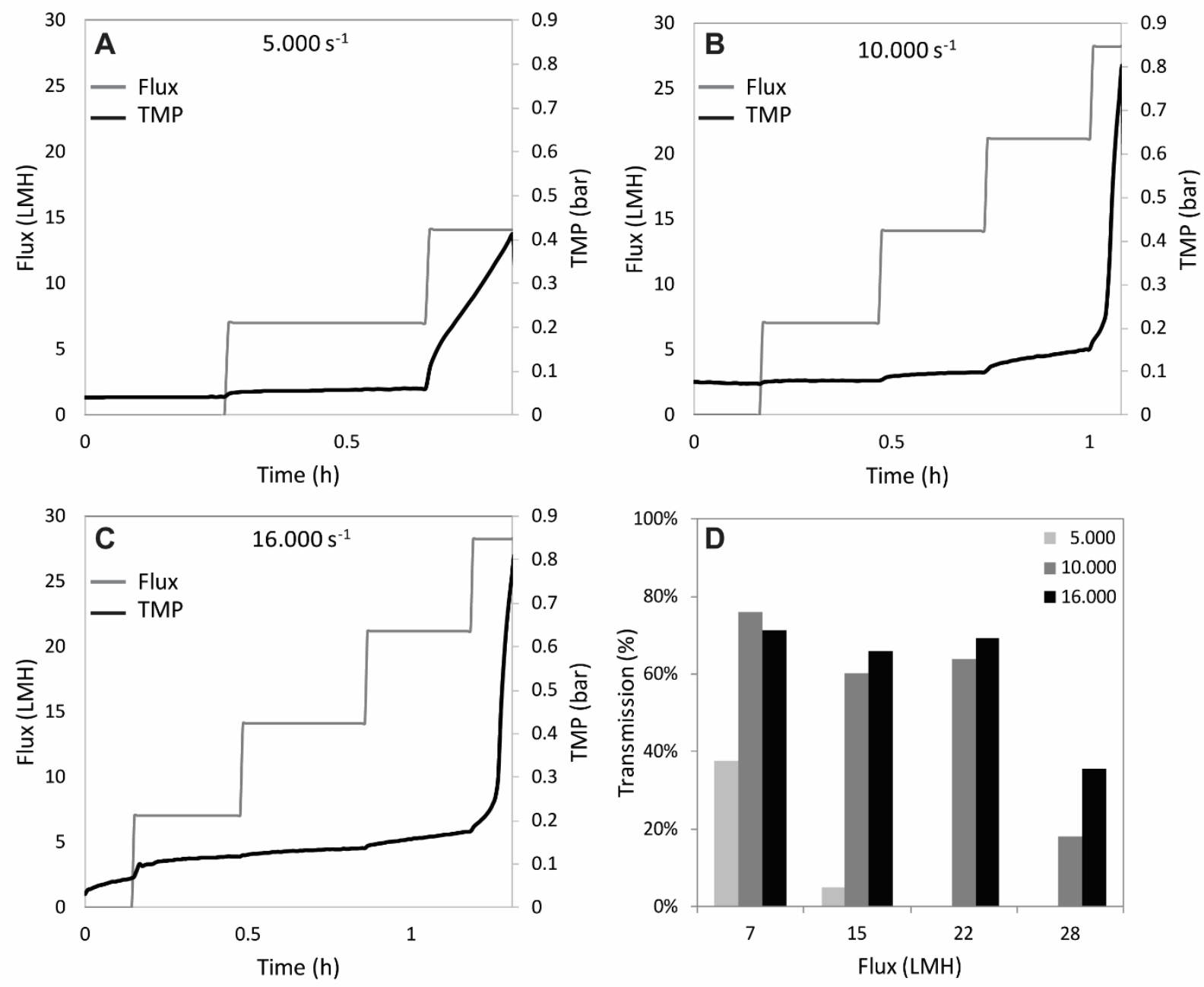

Figure 4. Flux excursion of the TFF of a dissolved oxygen induced OMV harvest. The performance of constant flux-mode processing of a high dissolved oxygen induced sOMV harvest was tested. The permeate flux (grey line) at increasing transmembrane pressures (black line) was monitored at shear rates of 5,000 s-1 (A), 10,000 s-1 (B), and 16,000 s-1 (C). The resulting sOMV transmission is shown (D).

expressing Nm strain. Western blot analysis showed that OspA was expressed for at least 20 generations after the end of the batch production culture (supplemental Figure 
1). All three strains, and the control strain without OspA and OspC, were grown in a batch culture with cysteine depletion in combination with high dissolved oxygen concentrations to trigger SOMV release. The growth of the four cultures was similar during the first 7 hours of cultivation (Figure 5). Then, a reduction in growth rate for the strains expressing heterologous antigens was observed resulting in a shift of $4 \mathrm{~h}(\mathrm{Nm}$ OspA, $\mathrm{Nm} \mathrm{OspC})$ and $7 \mathrm{~h}(\mathrm{Nm} \mathrm{OspA}+\mathrm{C})$ before reaching the maximum carbon dioxide evolution rate. Cultures were harvested $6 \pm 1 \mathrm{~h}$ after reaching the maximum carbon dioxide evolution rate, resulting in an average harvest of $2.0 \times 10^{15}$ sOMVs in $3 \mathrm{~L}$ culture. The optimized microfiltration process results in an average recovery of $90 \pm 17 \%$ of sOMVs. Next, the sOMVs were further purified using the same unit operations as the detergent-free purification process (data not shown). In brief, sOMVs were treated with DNAse, concentrated and diafiltrated. After clarification, the sOMVs were further purified using size-exclusion chromatography and filter sterilized.
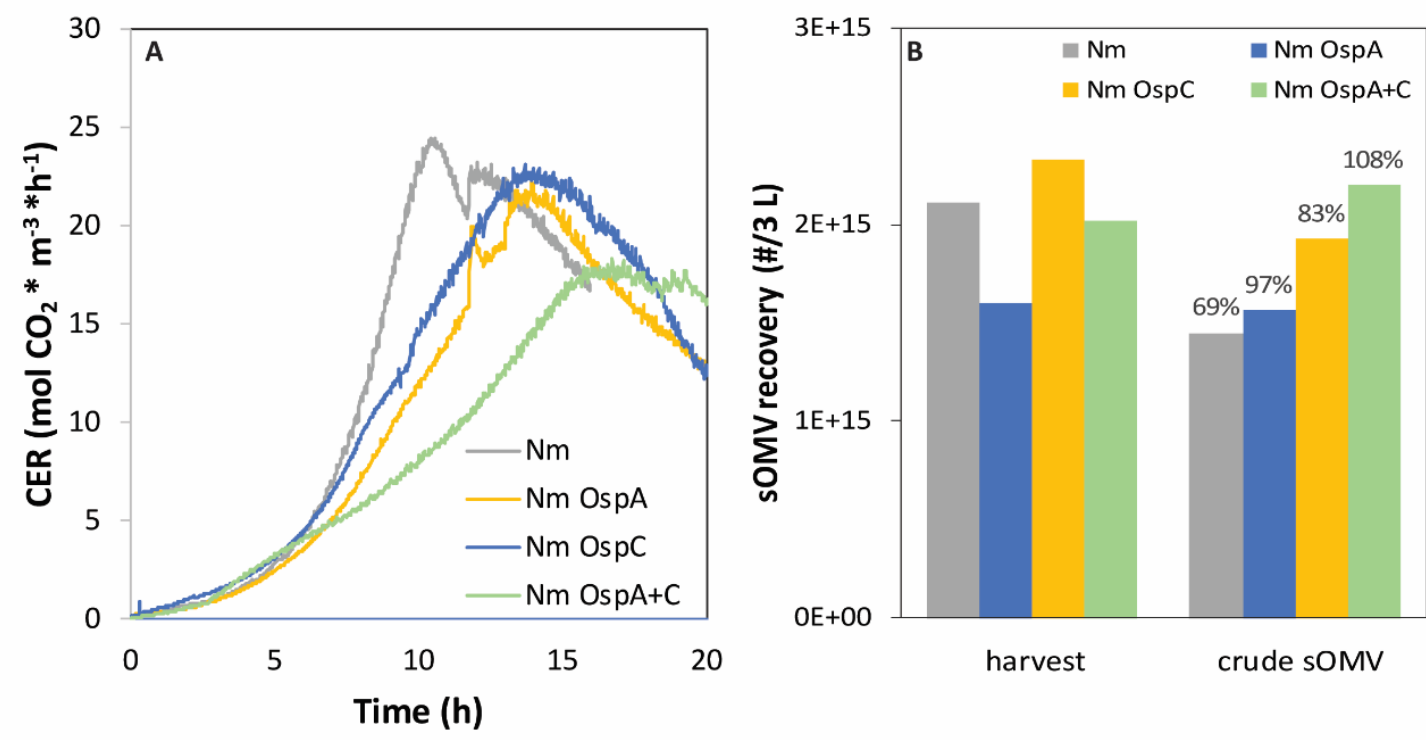

Figure 5. Bacterial growth and sOMV production of $N$. meningitidis expressing $B$. burgdorferi antigens. Bacterial growth of $\mathrm{Nm}$ strains expressing heterologous antigens in batch cultures at high oxygen concentrations is plotted (A) by the carbon dioxide evolution rate (CER). The respiratory quotient remained $1.0 \pm 0.05$ throughout the cultivation for all cultures (data not shown). The resulting amounts of sOMVs in the harvested culture (harvest) and after removal of bacterial cells (crude sOMV) are shown in panel B.

\section{sOMV characteristics}

The optimized sOMV production process was used to produce sOMVs and the effect of introducing different antigens on the characteristics of sOMVs was assessed. Purified 
sOMVs were highly similar in size distribution with a mean particle size of $105 \mathrm{~nm}$.

(Figure 6A). The average composition of purified OMVs also showed high similarities for the SOMVs containing heterologous antigens. The ratio of protein per vesicle and the ratio of LPS per vesicle was determined by combining concentration measurements of protein and LPS to the concentration of particles (Figure 6B). The protein pattern on an SDS-PAGE gel is also very similar (Figure 6C). The most abundant protein in all sOMVs is PorB (33.8 kDa). A minor amount of OspA (29.1 kDa) can be observed in the Nm OspA and Nm OspA + OspC OMVs. The fusion construct of OspC to fHbp (10.8 kDa) was not observed at the expected size in the Nm OspC sOMVs or the Nm OspA \& OspC sOMVs. However, analysis of the protein content by LC-MS/MS showed that OspC was nevertheless present in OMVs, although due to its small size and low concentration it was below the detection limit of SDS-PAGE. Based on LC-MS/MS, an estimated relative protein content of $0.04 \%(\mathrm{w} / \mathrm{w})$ and $0.07 \%(\mathrm{w} / \mathrm{w})$ OspC was retrieved for the $\mathrm{Nm}$ OspC and Nm OspA + OspC OMVs. The estimated relative content of OspA in Nm OspA and Nm OspA + OspC OMVs was $2.2 \%(w / w)$ and $0.31 \%(w / w)$. Since repetitive epitope presentation is required in proper vaccine function [39], we estimated the average number of OspA and OspC antigens per vesicle based on the particle and protein concentration and the relative abundance of the antigens. The strains expressing single antigens contain 55 and 2 molecules of OspA and OspC per OMV, respectively. Simultaneous expression yields 10 molecules of OspA and 4 molecules of OspC per OMV. Besides OspA and OspC, the overall protein composition of OMVs produced by the different production methods (eOMV, sOMV and sOMV by increased oxygen concentration) were compared. Based on the LC-MS/MS data the subcellular protein location of the OMV proteome was predicted by PSORTb. The sOMVs produced at high dissolved oxygen concentrations using the improved purification process are mainly composed of outer membrane proteins (55\%) and cytoplasmic proteins (25\%). The sOMVs produced at standard oxygen concentration show a comparable composition. However, eOMVs contain more cytoplasmic proteins (39\%) than outer membrane proteins (37\%) and are also enriched in cytoplasmic membrane associated proteins. Overall, eOMVs show a different protein composition to sOMVs. Expressing OspA or OspC on the surface of sOMVs does not impair the tested characteristics of the sOMVs. Furthermore, the results show that sOMVs formed at high dissolved oxygen 
concentrations have similar protein compositions to sOMVs produced at standard oxygen concentration.

\section{Discussion}

In this study, Nm sOMVs containing B. burgdorferi antigens were produced in a batch process by a purification process with scalable unit-operations. These heterologous antigens were expressed on the outer surface of the bacterium and were found in spontaneously released OMVs. SOMVs are advantageous over eOMVs production wise, since no extraction step is required in the production process. Here we applied high dissolved oxygen concentrations to bacterial cultures to stimulate sOMV production. Applying high dissolved oxygen concentrations to trigger SOMV release resulted in increased productivity but was lowering the recovery during microfiltration.

Microfiltration optimization showed that at constant TMP, sOMV transmission, but not protein transmission, is reduced in slightly fouling conditions. Using a larger pore size membrane and operating the tangential flow filtration at a constant permeate flux of $50 \%$ of the critical flux, improved the recovery to $90 \%$. sOMV quality attributes were highly consistent between the different antigen expressing strains, and highly comparable to sOMVs produced at standard oxygen concentrations. Compared to detergent-free extracted eOMVs, the sOMVs were enriched in outer membrane proteins. The amount of Nm sOMVs recovered in this study reached $2^{*} 10^{15}$ OMVs for a $3 \mathrm{~L}$ culture, corresponding to approximately $90 \mathrm{mg}$ of OMV associated protein per liter culture (mg/L). This is an improvement over $N$. meningitidis eOMV production, where 20 to $50 \mathrm{mg} / \mathrm{L}$ was reached [32], and N. meningitidis dOMV production where up to 22 $\mathrm{mg} / \mathrm{L}$ was reached $[40,41]$. It should be noted that the strains used in the other studies harbored different mutations than this strain. The parental strain, however, is the same. Other sOMV production described in literature include the Shigella sonnei sOMV yields of $140 \mathrm{mg} / \mathrm{L}$ [42], and 68 to $80 \mathrm{mg} / \mathrm{L}$ [43]. These yields were obtained from high-cell density cultures (OD600 35 to OD600 45), while this study is based on cultivation of up to 4 gram dry weight per liter culture (gdw/L). Interestingly a high cell density $N$. meningitidis perfusion cultivation has been described to reach a biomass concentration of $58 \mathrm{gdw} / \mathrm{L}$ [44], indicating that there is a possibility of process intensification if enhanced productivities are desired. 
Expressing heterologous antigens in $N$. meningitidis resulted in cultures with a lowered growth rate, however, production of sOMVs was maintained. More research is needed to assess the repeatability, the effect of additional antigens, and possibly the screening of clones or the use of an inducible production system could further improve the productivity of the heterologous antigens.

The number of antigens present on OMVs reported here are between 2 and 55 antigenic proteins per vesicle. These values were based on semi-quantitative LC-MS/MS protein composition measurements combined with nanoparticle tracking analysis of the $\mathrm{OMV}$ concentration and should thus be interpreted with some caution. Furthermore, the level of expression could have been influenced by the genomic location of the heterologous antigen as $O s p A$ was expressed by insertion in either rmpM or IpxL1. Hypothetically more antigens per vesicle would increase the potency of the composition, but it is
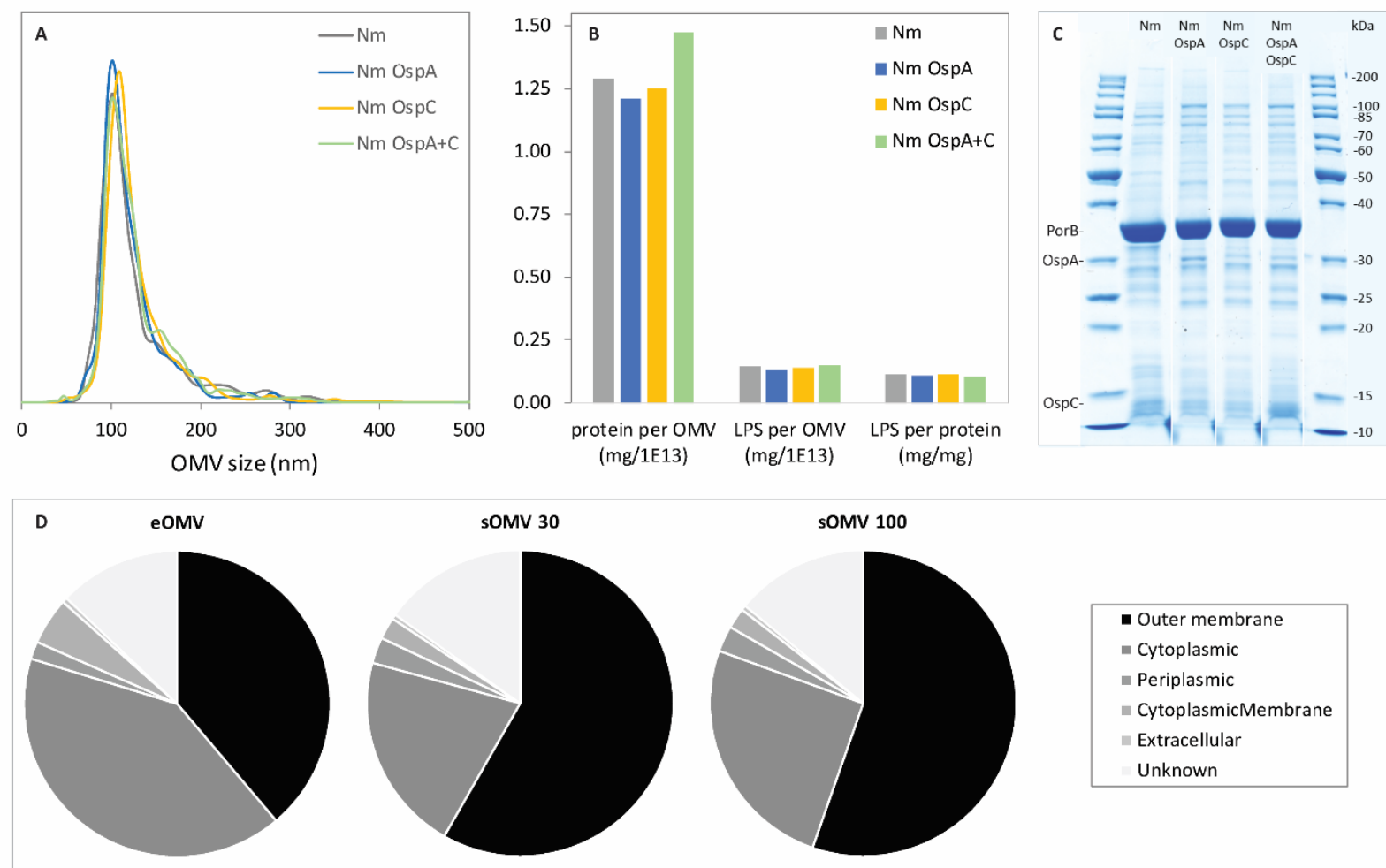

Figure 6 Characteristics of the OMVs. Nm sOMVs produced at high oxygen concentrations using the improved purification method were characterized. The size distribution of Nm sOMVs containing heterologous antigens shows a mode size of $105 \mathrm{~nm}(\mathrm{~A})$. The composition of the sOMVs is shown in amount of protein per OMV, amount of LPS per OMV and amount of LPS per protein (B). Protein composition of the sOMVs is analyzed by SDS-PAGE (C). Proteins from OMVs with different production methods (eOMV, sOMV by cysteine depletion (sOMV 30), sOMV by high dissolved oxygen (sOMV 100)) were measured by LC-MS/MS and their subcellular location was assessed by PSORTb (D). 
unknown what an optimum composition would be. Together, these design criteria are important aspects of future research.

In designing an OMV production process, SOMVs are advantageous over eOMVs from a process perspective. Here we show that the composition of eOMV differs from sOMV, but it is unknown what the effect of OMV type is on the immunogenicity for a vaccine platform. sOMVs are enriched in outer membrane proteins, which are usually immunogenic proteins. For a nonavalent PorA based Nm serogroup B vaccine, the crossprotection against PorA not included in the strain were assessed for $\mathrm{dOMV}$, eOMV and sOMV [26]. dOMVs did not show bactericidal titers against strains not included in the vaccine, whereas eOMVs showed some cross-protection against strains expressing PorA not included in the vaccine. SOMVs did elicit broad cross protection against all tested strains and a minor response against a strain lacking PorA. sOMVs are thus advantageous over eOMV and dOMV for vaccine compositions providing protection against the OMV-producing strain. However, further research is required to elucidate the differences between the type of OMV on the efficacy of an OMV-based vaccine platform.

In this platform design we use a pathogenic bacterium as production host, in contrast to common production bacteria that are closely related to commensal bacteria. This approach ensures that the vaccine platform does not cause unwanted responses against commensal bacteria. Another concern of vaccine platforms is the possible response against the platform itself, possibly causing immune dominance of the carrier. Currently it is unknown if reduction of the antigenicity of the OMV-backbone is required to yield an effective vaccine platform. Here we aimed to reduce the antigenic content of the backbone by removing the capsular polysaccharide, LPS and the antigen outer membrane porin A (PorA). However, the OMVs showed to be enriched in another porin, PorB. Nm PorB is a TLR-2 ligand [45], that may be advantageous because of its adjuvating function [46]. The observation of a large amount of PorB in sOMVs is contrary to the proteome of Nm sOMVs described by Lappann et al., where sOMVs were found to be reduced in PorA and PorB [47], which bind to the periplasmic protein RmpM [48]. Here we used a Nm strain without RmpM to enhance OMV release, which could explain the increase of PorB, since linkage of PorB with RmpM and the peptidoglycan is not possible in this strain. 
Overall, this study demonstrates the feasibility of the production of an OMV-based vaccine platform based on the expression of heterologous antigens on N. meningitidis sOMV. We showed that the addition of two Lyme disease antigens affected the growth, but yielded similar amounts of OMVs with similar characteristics as the Nm OMV control. Purification of sOMVs was possible by optimizing the purification process. By applying high oxygen concentrations to the bacteria, yields of $90 \mathrm{mg} / \mathrm{L}$ highly pure OMVs were obtained. With this process it becomes feasible to produce sufficient $N$. meningitidis OMVs with various heterologous antigens and study their potential as future vaccines.

\section{Acknowledgements}

The authors thank Lilli Stangowez and Lonneke van Keulen for their help in performing the sOMV productions, Joost Uittenbogaard and Alex de Haan for the Mass Spectroscopy and phospholipid analysis, and Gideon Kersten and Leo van der Pol for critical reading of the manuscript. This work has been funded by the Ministry for Health, Welfare and Sports (The Netherlands).

\section{References}

1. Ellis, T.N. and M.J. Kuehn, Virulence and Immunomodulatory Roles of Bacterial Outer Membrane Vesicles. Microbiology and Molecular Biology Reviews, 2010. 74(1): p. 81-94.

2. Kesty, N.C. and M.J. Kuehn, Incorporation of Heterologous Outer Membrane and Periplasmic Proteins into Escherichia coli Outer Membrane Vesicles. The Journal of biological chemistry, 2004. 279(3): p. 20692076.

3. Gnopo, Y.M.D., et al., Designer outer membrane vesicles as immunomodulatory systems - Reprogramming bacteria for vaccine delivery. Advanced Drug Delivery Reviews, 2017. 114: p. 132-142.

4. Pritsch, M., et al., Comparison of Intranasal Outer Membrane Vesicles with Cholera Toxin and Injected MF59C.1 as Adjuvants for Malaria Transmission Blocking Antigens AnAPN1 and Pfs48/45. J Immunol Res, 2016. 2016: p. 3576028.

5. Lastre, M., et al., Bacterial derived proteoliposome for allergy vaccines. Vaccine, 2006. 24 Suppl 2: p. S234-5.

6. Mitra, S., M.K. Chakrabarti, and H. Koley, Multi-serotype outer membrane vesicles of Shigellae confer passive protection to the neonatal mice against shigellosis. Vaccine, 2013. 31(31): p. 3163-73.

7. Grandi, A., et al., Synergistic Protective Activity of Tumor-Specific Epitopes Engineered in Bacterial Outer Membrane Vesicles. Front Oncol, 2017. 7: p. 253.

8. Kaparakis-Liaskos, M. and R.L. Ferrero, Immune modulation by bacterial outer membrane vesicles. Nat Rev Immunol, 2015. 15(6): p. 375-87.

9. Tang, B., et al., Effect of outer membrane vesicles derived from Escherichia coli on proliferation, apoptosis and migration of human neuroblastoma SK-N-SH cells in vitro. Nan Fang Yi Ke Da Xue Xue Bao, 2018. 38(3): p. 334-339.

10. Gerritzen, M.J.H., et al., Bioengineering bacterial outer membrane vesicles as vaccine platform. Biotechnology Advances, 2017. 35(5): p. 565-574.

11. Zariri, A. and P. van der Ley, Biosynthetically engineered lipopolysaccharide as vaccine adjuvant. Expert Rev Vaccines, 2015. 14(6): p. 861-76.

12. Serruto, D., et al., The new multicomponent vaccine against meningococcal serogroup $B$, 4CMenB: immunological, functional and structural characterization of the antigens. Vaccine, 2012. 30 Suppl 2: p. B87-97. 
13. Bacon, R.M., et al., Surveillance for Lyme disease--United States, 1992-2006. MMWR Surveill Summ, 2008. 57(10): p. 1-9.

14. Steere, A.C., 34 - Lyme Disease Vaccines A2 - Plotkin, Stanley A, in Plotkin's Vaccines (Seventh Edition), W.A. Orenstein, P.A. Offit, and K.M. Edwards, Editors. 2018, Elsevier. p. 549-560.e5.

15. Comstedt, P., et al., The novel Lyme borreliosis vaccine VLA15 shows broad protection against Borrelia species expressing six different OspA serotypes. PLoS One, 2017. 12(9): p. e0184357.

16. Wressnigg, N., et al., Safety and immunogenicity of a novel multivalent OspA vaccine against Lyme borreliosis in healthy adults: a double-blind, randomised, dose-escalation phase 1/2 trial. Lancet Infect Dis, 2013. 13(8): p. 680-9.

17. Sikand, V.K., et al., Safety and immunogenicity of a recombinant Borrelia burgdorferi outer surface protein A vaccine against lyme disease in healthy children and adolescents: a randomized controlled trial. Pediatrics, 2001. 108(1): p. 123-8.

18. Fantappiè, L., et al., Antibody-mediated immunity induced by engineered Escherichia coli OMVs carrying heterologous antigens in their lumen. Journal of Extracellular Vesicles, 2014. 3: p. 10.3402/jev.v3.24015.

19. Muralinath, M., et al., Immunization with Salmonella enterica Serovar Typhimurium-Derived Outer Membrane Vesicles Delivering the Pneumococcal Protein PspA Confers Protection against Challenge with Streptococcus pneumoniae. Infection and Immunity, 2011. 79(2): p. 887-894.

20. Schild, S., et al., Characterization of Vibrio cholerae Outer Membrane Vesicles as a Candidate Vaccine for Cholera. Infection and Immunity, 2009. 77(1): p. 472-484.

21. Bartolini, E., et al., Recombinant outer membrane vesicles carrying Chlamydia muridarum HtrA induce antibodies that neutralize chlamydial infection in vitro. Journal of Extracellular Vesicles, 2013. 2: p. 10.3402/jev.v2i0.20181.

22. Salverda, M.L.M., et al., Surface display of a borrelial lipoprotein on meningococcal outer membrane vesicles. Vaccine, 2016. 34(8): p. 1025-1033.

23. van de Waterbeemd, B., et al., Cysteine depletion causes oxidative stress and triggers outer membrane vesicle release by Neisseria meningitidis; implications for vaccine development. PLoS One, 2013. 8(1): p. e54314.

24. Gerritzen, M.J.H., et al., High dissolved oxygen tension triggers outer membrane vesicle formation by Neisseria meningitidis. Manuscript submitted for publication, 2018.

25. Holten, E., Serotypes of Neisseria meningitidis isolated from patients in Norway during the first six months of 1978. J Clin Microbiol, 1979. 9(2): p. 186-8.

26. van de Waterbeemd, B., et al., Improved OMV vaccine against Neisseria meningitidis using genetically engineered strains and a detergent-free purification process. Vaccine, 2010. 28(30): p. 4810-6.

27. van der Ley, P., et al., Modification of lipid A biosynthesis in Neisseria meningitidis lpxL mutants: influence on lipopolysaccharide structure, toxicity, and adjuvant activity. Infect Immun, 2001. 69(10): p. 5981-90.

28. Steeghs, L., et al., Neisseria meningitidis expressing lgtB lipopolysaccharide targets DC-SIGN and modulates dendritic cell function. Cell Microbiol, 2006. 8(2): p. 316-25.

29. Tommassen, J., et al., Isolation of Neisseria meningitidis mutants deficient in class 1 (porA) and class 3 (porB) outer membrane proteins. Infect Immun, 1990. 58(5): p. 1355-9.

30. Pajon, R., A.M. Fergus, and D.M. Granoff, Mutant Native Outer Membrane Vesicles Combined with a Serogroup A Polysaccharide Conjugate Vaccine for Prevention of Meningococcal Epidemics in Africa. PLoS One, 2013. 8(6): p. e66536.

31. Baart, G.J., et al., Modeling Neisseria meningitidis metabolism: from genome to metabolic fluxes. Genome Biol, 2007. 8(7): p. R136.

32. van de Waterbeemd, B., et al., Improved production process for native outer membrane vesicle vaccine against Neisseria meningitidis. PLoS One, 2013. 8(5): p. e65157.

33. Malloy, A. and B. Carr, NanoParticle Tracking Analysis - The Halo ${ }^{\text {TM }}$ System. Particle \& Particle Systems Characterization, 2006. 23(2): p. 197-204.

34. Gerritzen, M.J.H., et al., High throughput nanoparticle tracking analysis for monitoring outer membrane vesicle production. Journal of Extracellular Vesicles, 2017. 6(1): p. 1333883.

35. Jantzen, E., T. Bergan, and K. Bovre, Gas chromatography of bacterial whole cell methanolysates; VI. Fatty acid composition of strains within Micrococcaceae. Acta Pathol Microbiol Scand B Microbiol Immunol, 1974. 82(6): p. 785-98.

36. Welch, D.F., Applications of cellular fatty acid analysis. Clinical Microbiology Reviews, 1991. 4(4): p. 422438.

37. Meiring, H.D., et al., Nanoscale LC-MS(n): technical design and applications to peptide and protein analysis. Journal of Separation Science, 2002. 25(9): p. 557-568.

38. Yu, N.Y., et al., PSORTb 3.0: improved protein subcellular localization prediction with refined localization subcategories and predictive capabilities for all prokaryotes. Bioinformatics, 2010. 26(13): p. 1608-15. 
39. Hinton, H.J., A. Jegerlehner, and M.F. Bachmann, Pattern Recognition by B Cells: The Role of Antigen Repetitiveness Versus Toll-Like Receptors, in Specialization and Complementation of Humoral Immune Responses to Infection, T. Manser, Editor. 2008, Springer Berlin Heidelberg: Berlin, Heidelberg. p. 1-15.

40. Fredriksen, J.H., et al., Production, characterization and control of MenB-vaccine "Folkehelsa": an outer membrane vesicle vaccine against group B meningococcal disease. NIPH Ann, 1991. 14(2): p. 67-79; discussion 79-80.

41. Claassen, I., et al., Production, characterization and control of a Neisseria meningitidis hexavalent class 1 outer membrane protein containing vesicle vaccine. Vaccine, 1996. 14(10): p. 1001-1008.

42. Scorza, F.B., et al., High Yield Production Process for Shigella Outer Membrane Particles. PLOS ONE, 2012. 7(6): p. e35616.

43. Gerke, C., et al., Production of a Shigella sonnei vaccine based on generalized modules for membrane antigens (GMMA), 1790GAHB. PloS one, 2015. 10(8): p. e0134478.

44. Dehottay, P.M.H., et al., Fermentation of fastidious bacterial strain in perfusion suspension culture. 2014, GLAXOSMITHKLINE BIOLOGICALS S.A.

45. Singleton, T.E., P. Massari, and L.M. Wetzler, Neisserial porin-induced dendritic cell activation is MyD88 and TLR2 dependent. J Immunol, 2005. 174(6): p. 3545-50.

46. Reiser, M.L., et al., The TLR2 Binding Neisserial Porin PorB Enhances Antigen Presenting Cell Trafficking and Cross-presentation. Scientific Reports, 2017. 7(1): p. 736.

47. Lappann, M., et al., Comparative proteome analysis of spontaneous outer membrane vesicles and purified outer membranes of Neisseria meningitidis. Journal of Bacteriology, 2013.

48. Maharjan, S., et al., Dissection of the function of the RmpM periplasmic protein from Neisseria meningitidis. Microbiology, 2016. 162(2): p. 364-375.

Supplemental Figure 1. Stability of heterologous expression OspA on $\mathbf{N}$. meningitidis. The genetic stability of expressing a heterologous antigen in Nm OMVs was assessed. Bacterial culture samples (whole cells) show by Western blot analysis the presence of OspA in the master frozen seedlot (MSL), working frozen seedlot (WSL), preculture (PC), production culture (BR), and subcultures after the end of the batch production culture (SF3-SF6). The OspA construct was detected in all samples at the expected size (29.1 kDa), proving the stability of the expression for at least 20 generations after the production culture. The amount of sample loaded varied between samples causing differences in the intensity of the OspA-band.

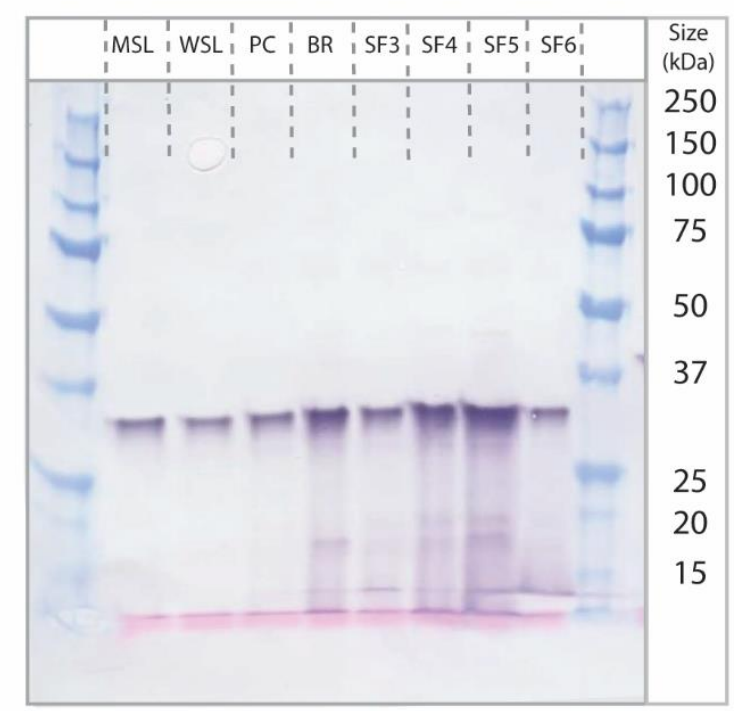




\section{Chapter 7}

\section{Continuous production of Neisseria meningitidis outer membrane vesicles}

Submitted for publication:

Gerritzen, M.J.H., L. Stangowez. B. van de Waterbeemd, D.E. Martens, R.H. Wijffels and M. Stork. " Continuous production of Neisseria meningitidis outer membrane vesicles." 


\begin{abstract}
Outer membrane vesicles (OMVs) are nanoparticles secreted by Gram-negative bacteria that can be used for diverse biotechnological applications. Interesting applications have been developed, where OMVs are the basis of drug delivery, enzyme carriers, adjuvants, and vaccines. Historically, OMV research has mainly focused on vaccines. Therefore, current OMV production processes have been based on batch processes. The production of OMVs in batch mode is characterized by relatively low yields and high costs. Transition of OMV production processes from batch to continuous processes could increase the volumetric productivity, reduce the production and capital costs, and result in a higher quality product. Here we study the continuous production of $N$. meningitidis OMVs to improve volumetric productivity. Continuous cultivation of $N$. meningitidis resulted in a steady state with similar high OMV concentrations as are reached in current batch processes. The steady state was reproducible and could be maintained for at least 600 hours. The volumetric productivity of a continuous culture reached $4.0 \times 10^{14}$ OMVs per liter culture per day, based on a dilution rate of $1 /$ day. The tested characteristics of the OMVs did not change during the experiments showing feasibility of a continuous production process for the production of OMVs for any application.
\end{abstract}




\section{Introduction}

Outer membrane vesicles (OMVs) are nanoparticles produced by Gram-negative bacteria. OMVs are non-replicative and consist of lipopolysaccharides (LPS), phospholipids, and periplasmic and membrane-bound proteins [1]. Biologically, OMVs play roles in intercellular communication, competition, virulence, and nutrient acquisition [2]. In biotechnology, OMVs can be used as enzyme carriers [3], as drug delivery vehicles [4], as vaccines [5], or as adjuvants [6].

Most research on OMVs has focused on vaccine development. Especially the development of OMV-based vaccines for serogroup B Neisseria meningitidis (Nm) has been researched extensively [7,8]. Nm OMVs have been successfully used in outbreaks of meningococcal disease [9-11]. Moreover, OMVs are included in the currently available serogroup B vaccine Bexsero [12]. Traditionally, the production processes of Nm OMV vaccines have been based on the extraction of vesicles from biomass using detergents (dOMV). This was necessary to reduce the endotoxicity of Nm LPS. Genetic detoxification of Nm LPS allowed the use of detergent-free extracted OMVs as well as spontaneously released OMVs (sOMVs) [13]. sOMVs are released by $N$. meningitidis, and other bacteria, during normal growth, without the use of any detergents as used for dOMVs or chelating agents as used for extracted OMVs (eOMVs). Compared to both dOMVs and eOMVs, the sOMVs have a different biochemical composition and superior immunogenicity [14]. However, the yield of sOMV production processes is currently too low for feasible vaccine production. If the sOMV yield could be improved, sOMVs are the preferred basis for vaccine development, instead of dOMVs or eOMVs. Additionally, sOMVs can be purified with less unit operations as the vesicles can be directly purified from the supernatants of bacterial cultures without any extraction steps. Besides the use as vaccines, high-yield sOMV production processes would allow the use of sOMVs for other biotechnological applications.

Recently, we showed high yields of sOMV from a batch cultivation [15]. This was reached by using a production strain with reduced linkage of the outer membrane to the peptidoglycan in combination with sulfur source depletion [16], and high dissolved oxygen levels [17]. Cysteine depletion triggers OMV release [16, 18]. However, this mild stress also results in growth arrest and accumulation of undesired components, like 
DNA that complicates filtration and ammonium that inhibits the nuclease required for DNA removal [18].

Despite the recent developments for improved OMV production processes, higher yields are required to facilitate the use of OMVs as low-cost vaccines, adjuvant, enzyme carrier or drug delivery vehicle. Continuous bioprocessing can be a solution for high yield OMV production. Continuous bioprocessing can result in higher equipment utilization rates, reduced cycle times, and smaller facility footprints [19], which in turn results in lower production and investment costs. Conversion of batch processes to continuous processes has already shown to be an improvement in many industries [20]. The potential of continuous bioprocessing for the production of biopharmaceuticals shows great potential and has been recognized as a paradigm shift in biologicals production [20]. Additionally, development of continuous biopharmaceutical production has been encouraged by the FDA $[21,22]$. The aim of this study is to assess the use of steady-state chemostat cultures as an upstream process for continuous processing to obtain a high volumetric productivity of Nm sOMVs. We first assess the production of OMVs in a continuous culture. Then, we further characterize the pre-steady-state phase of the culture and the reproducibility of OMV production was assessed by comparing five replicate steady state continuous cultures. Optimization of the volumetric productivity was assessed by testing different dilution rates. Lastly, we compare continuous OMV production to the production of OMVs in batch and fed-batch cultures.

\section{Methods}

\section{Bacterial strain}

A derivative of the H44/76 isolate of Neisseria meningitidis serogroup B [23] was used as described previously [24]. In brief, the strain was non-encapsulated due to a siaD knockout [25], and has reduced LPS-toxicity from an IpxL1 deletion. This strain has further improved vesicle formation due to the rmpM deletion, lacks the major abundant outer membrane protein PorA [26], and has improved interaction with dendritic cells by $\lg t B$ deletion [27].

\section{Chemostat cultures}

Continuous cultures with a working volume of 2 liter were performed in 5-liter benchtop bioreactors (Applikon) with an H/D ratio of 1.6 based on total volume. The culture medium was chemically defined without animal derived components containing 
glucose, amino acids, salts, iron, and trace elements [28]. The reactors were controlled using a Tryton ${ }^{\mathrm{i}}$ (Pierre Guerin), that controlled the temperature at $35 \pm 0.5^{\circ} \mathrm{C}$ and $\mathrm{pH}$ at pH $7.2 \pm 0.05$ using $1 \mathrm{M} \mathrm{HCl}$ and $1 \mathrm{M} \mathrm{NaOH}$. Dissolved oxygen tension was measured using polarographic oxygen sensors (InPro 6850i, Mettler Toledo) that were calibrated at $100 \%$ in air-saturated medium of $35^{\circ} \mathrm{C}$. The cultivations were controlled at $30 \%$ air saturation by increasing agitation rate in the batch phase of the cultivation (300-1000 RPM) and mixing of oxygen and air in the headspace aeration (fixed flow rate of 1 $\mathrm{L} / \mathrm{min}$ ). The off-gas composition was measured by a mass-spectrometer (Prima $\delta \mathrm{b}$, Thermo Scientific). Feed and bleed pumps were started after $8 \pm 2$ hours of growth to initiate a continuous culture. The bioreactor weight, the feed medium weight and the $\mathrm{pH}$ titrant solutions were measured by balances to verify the dilution rate. The dilution rate was set to $0.04 \mathrm{~h}^{-1}$ unless indicated otherwise. Culture samples were analyzed for biomass density by measuring the optical density at $590 \mathrm{~nm}$. Steady state was assumed after 3 dilutions based on steady bacterial density measurements and carbon dioxide emission.

\section{Analytical}

Filtered culture samples $(0.22 \mu \mathrm{m}$ pore-size $)$ were measured by Nanoparticle tracking analysis on a NanoSight NS500 with $488 \mathrm{~nm}$ laser module and sCMOS camera [29]. Temperature was controlled at $25^{\circ} \mathrm{C}$ and measurements (10 captures of 30 -seconds) were analyzed with the NTA 3.2 software build 3.2.16. Measurements were taken under flow using the automated script described previously [24]. Residual genomic DNA was determined with a dsDNA assay based on fluorescence. In brief, sterile filtered culture samples were incubated with Quant-iT PicoGreen dsDNA reagent (Invitrogen), and fluorescence was measured to quantify the DNA concentration based on a calibration curve with salmon sperm DNA standard (Invitrogen).

\section{Nutrient and metabolite analysis}

Amino acids in culture supernatants were measured using the method based on derivatization by orthophtalic anhydride and High Performance Liquid Chromatography (HPLC) as described in $[14,30]$. Organic acids were analyzed by HPLC on a Waters Acquity Class-H (Waters) HPLC system that was equipped with an Acclaim Organic acid guard column ( 3 x $10 \mathrm{~mm}, 5 \mu \mathrm{m}$, Thermo Scientific) and a Luna ${ }^{\circledR}$ Omega 3 um polar C18 $100 \AA 4.6 \mathrm{~mm}$ x $150 \mathrm{~mm}$ LC column (Phenomenex). The eluent was 0.1 
$\mu \mathrm{m}$ sterile filtered $20 \mathrm{mM}$ Potassium phosphate pH $2.520 \mathrm{mM}$, set to $\mathrm{pH} 2.5$ using $4 \mathrm{M}$ $\mathrm{HCl}$. Isocratic elution was performed at $1 \mathrm{~mL} / \mathrm{min}$ followed by UV (210 $\mathrm{nm})$ and refractive index detection. Samples from bacterial culture supernatants, standards and controls were first $0.22 \mu \mathrm{m}$ filtered, to the filtered supernatant (284 $\mu \mathrm{L})$ concentrated phosphoric acid (10 $\mu \mathrm{L}, 14.7 \mathrm{M}$ ) containing propionic acid as ISTD was added. Samples were mixed vigorously and subsequently centrifuged $10 \mathrm{~min}, 15.000 \mathrm{xg}$ (Thermo Scientific MicroCL 21R) at ambient temperature. Supernatants were collected and analyzed for organic acid content. Organic acid contents were compared to a standard mixture containing L-Glutamic acid, D-(-)-Tartaric acid, D-(+)-Malic acid, L-(+)-Lactic acid, Citric acid, Acetic acid, Succinic acid, Fumaric acid of which a calibration curve was constructed. All chromatographic parameters were calculated using the Chromeleon software (v. 7.2, SR 8, Thermo Fisher Scientific). Ammonium was measured in sterile filtered samples using the BioProfile 100 plus (Nova Biomedical). Glucose concentration was determined by ${ }^{1} \mathrm{H}-\mathrm{NMR}$ as previously described [31].

\section{SDS-PAGE}

For the protein analysis, OMVs were purified from culture samples by initial removal of biomass by centrifugation at $3000 \mathrm{xg}$ for 20 minutes at $4^{\circ} \mathrm{C}$. Next, the supernatant was sterile filtered using a Nalgene ${ }^{\mathrm{TM}}$ Rapid-Flow ${ }^{\mathrm{TM}}$ filter unit (Thermo Fisher Scientific) containing a PES membrane with a $0.2 \mu \mathrm{m}$ cut-off. Then, the sterile filtrate pool was concentrated 10 - 15 times using Amicon Ultra-15 Centrifugal filter units (Merck Millipore) at 4000xg for 40 minutes at room temperature. Finally, the concentrated filtrate was diafiltrated, in the same unit, with 2 volumes of buffer (100 mM Tris- $\mathrm{HCl}, \mathrm{pH}$ 8.6) by centrifugation (4000xg, 15 minutes, room temperature). Purified OMVs were assessed for total protein content by the Lowry protein assay using Peterson's modification. OMVs corresponding to $4 \mu \mathrm{g}$ of protein were loaded on a precast polyacrylamide gel (Lonza) to perform SDS-gel electrophoresis. The electrophoresis was run at $140 \mathrm{~V}$ for 90 minutes with Accugene 1x Tris-Glycine SDS buffer. To determine the molecular weight a Pierce ${ }^{\mathrm{TM}}$ pre-stained protein weight marker (Thermo Fisher Scientific) was used. The staining of the gel was performed by InstantBlue protein stain (Expedeon) for 1 hour. 


\section{Results}

\section{Continuous cultivation of $N$. meningitidis}

A chemostat culture for the continuous production of Nm sOMVs was started as a batch culture. When the mid exponential growth phase was reached ( 8 hours), the dilution was started at a rate of 1/day. A steady state was reached after 100 hours, which equals 4 dilutions of the culture (Figure 1). This steady state could be maintained for at least 600 hours. The steady state biomass concentration was $0 D_{590 \mathrm{~nm}}=14 \pm 2$. Interestingly, steady state OMV concentrations reached $4 \times 10^{11} / \mathrm{mL}$, which is similar to the maximum sOMV concentration reached in batch cultures in which OMV release was triggered by cysteine depletion $[17,32]$.

A

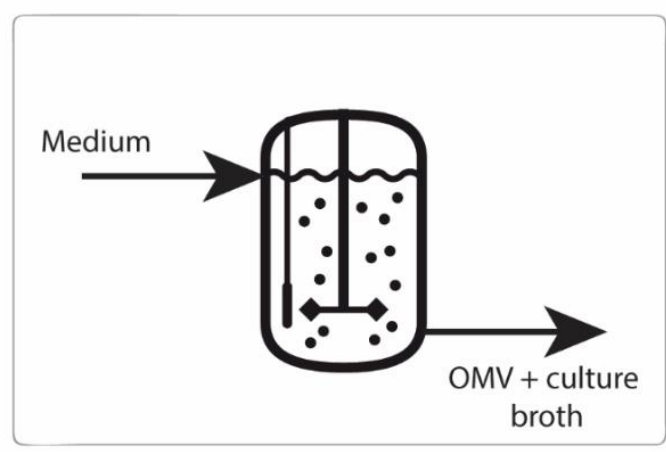

C

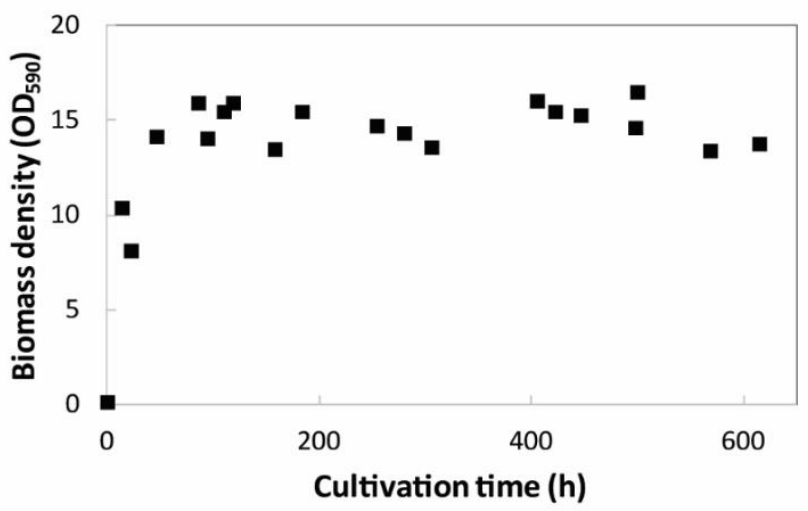

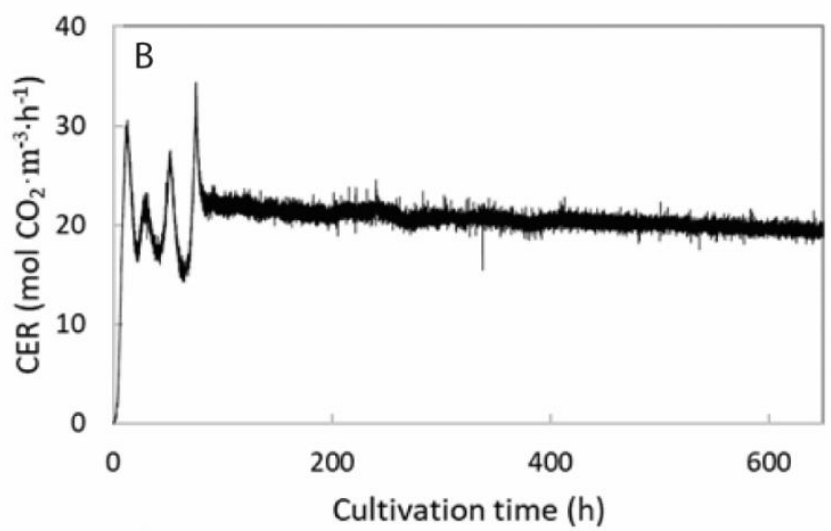

$\mathrm{D}$

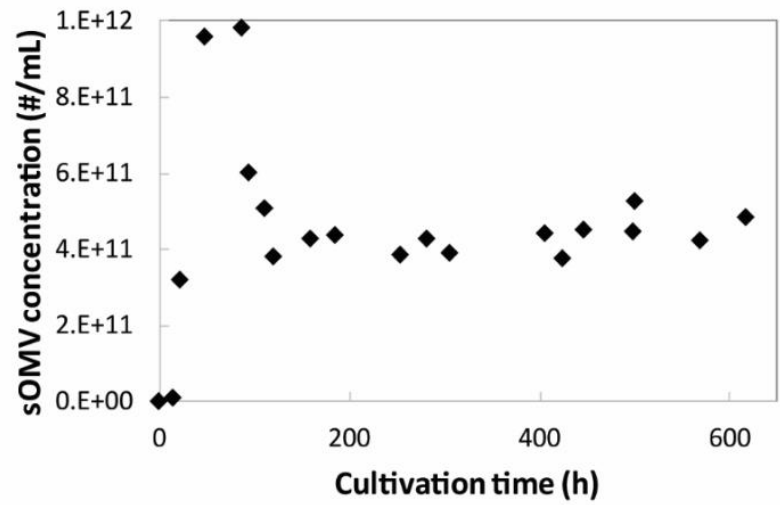

Figure 1 Continuous production of $N$. meningitidis OMVs in a chemostat at a dilution rate of $0.04 \mathrm{~h}^{-}$

1. A: schematic presentation of the chemostat setup showing continuous dilution of the bioreactor with fresh culture medium (graph A). Steady state was reached after 100 hours, as shown by the steady carbon dioxide evolution rate (CER) (graph B), biomass density (graph C), and the OMV concentration (graph D). The culture shown is representative for the 5 replicates that were done. 
The time to reach the steady state of the culture was characterized by periods of faster and slower growth. We hypothesize that this is a result of the complexity of the growth medium that contains glucose, glutamate, arginine, cysteine, and sulfate. The culture was fed a balanced medium composition that has been designed for batch cultivations [31]. We assume that the periods of slower and faster growth correlate with certain substrates becoming limiting and an induction period to switch to alternative substrates. Duplicate continuous cultures were performed to analyze nutrient utilization during the pre-steady state period. The cultures reached steady state based on carbon dioxide emission after approximately 4 dilutions (100 h) of the reactor (Figure 2). The growth of the replicates and the obtained steady state was similar to each other and to the culture shown in Figure 1. After 8 hours of exponential growth, dilution of the culture was started. At this point, cysteine is the first nutrient to be depleted (Supplemental Figure 1). In the next phase, sulfate was sufficiently available and presumably used for biosynthesis of cysteine. Additionally, arginine was depleted, after which $\mathrm{Nm}$ is capable of arginine biosynthesis from glutamate. Next, the glutamate concentrations decrease below $20 \mathrm{mM}$ (16 h), after which glutamate concentrations stabilized as well as the carbon dioxide emission. From this moment, significant amounts of malic acid, tartaric acid and acetic acid have accumulated. After 36 hours the carbon dioxide emission increased again and after 50 hours of cultivation glucose was depleted. Upon glucose limitation the residual glutamate was consumed and a steady state was reached. In this steady state the biomass yield on glucose and on glutamate is respectively $0.42 \pm 0.04 \mathrm{gdw}_{\mathrm{dw}} / \mathrm{gglucose}$ and $0.37 \pm 0.05 \mathrm{~g}_{\mathrm{dw}} / \mathrm{g}_{\text {glutamate. }}$. Based on the glucose and glutamate depletion we assumed that the steady state was limited by the carbonsource. To assess this limitation, a steady state culture was supplemented with a bolus feed of glucose to increase the glucose concentration in the reactor with $20 \mathrm{mM}$ (supplemental Figure 2). In the first hour after addition, a decrease in bacterial density was observed that is caused by the volume of the addition. After this, an increase in bacterial growth was observed by an increasing optical density and increased carbon dioxide emission after this addition, indicating that the steady state culture is glucoselimited. 

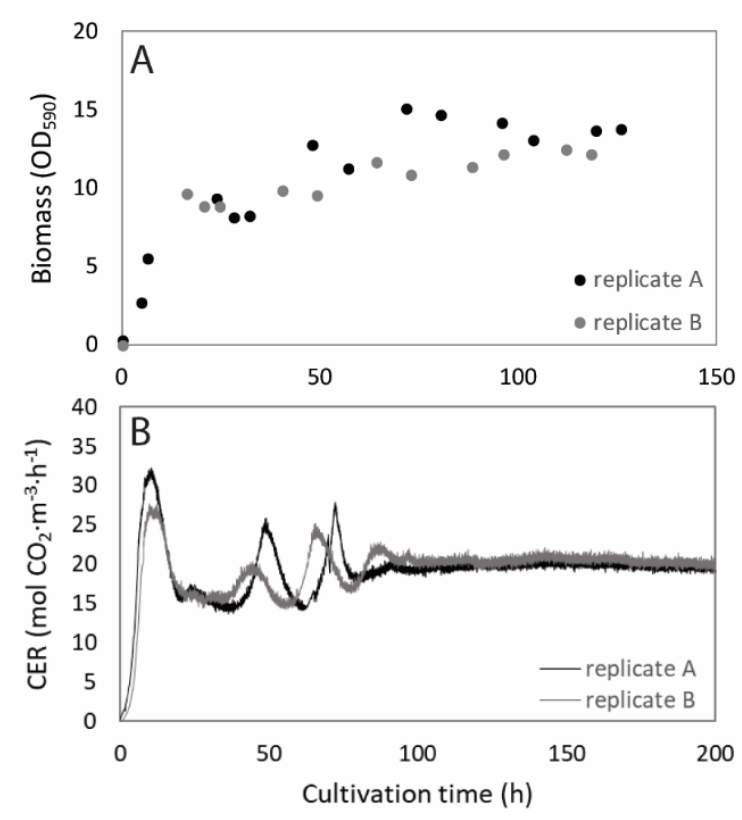

Figure 2. Pre-steady-state of the continuous cultivation. Two replicate chemostat cultures were started to characterize the pre-steady state phase. Graph A shows the biomass concentration measured by optical density measurements and the carbon dioxide evolution rate (CER) is shown in Graph B.

\section{Steady state reproducibility}

The reproducibility of the obtained steady state was assessed by comparing 5 replicate cultures (Figure 3). Culture to culture variability is low as indicated by the standard deviation of the carbon dioxide emission rate. A small decreasing trend can be observed that is caused by a lowered inflow of feed medium due to peristaltic pump tubing wear. Additionally, the bioreactor volume decreases slightly in time. Biomass concentrations are reproducible between cultures. A small increasing trend in biomass concentration can be observed that is caused by the altered bioreactor dilution rate because of the changes in the bioreactor volume and medium inflow mentioned earlier. Steady state OMV concentrations were on average $4 \times 10^{11} / \mathrm{mL}$. Steady state OMV productivity was maintained for at least $500 \mathrm{~h}$ (20 dilutions). OMVs were purified from two of the replicates at different time-points in the culture and showed similarity based on their protein composition (Figure 3D). 

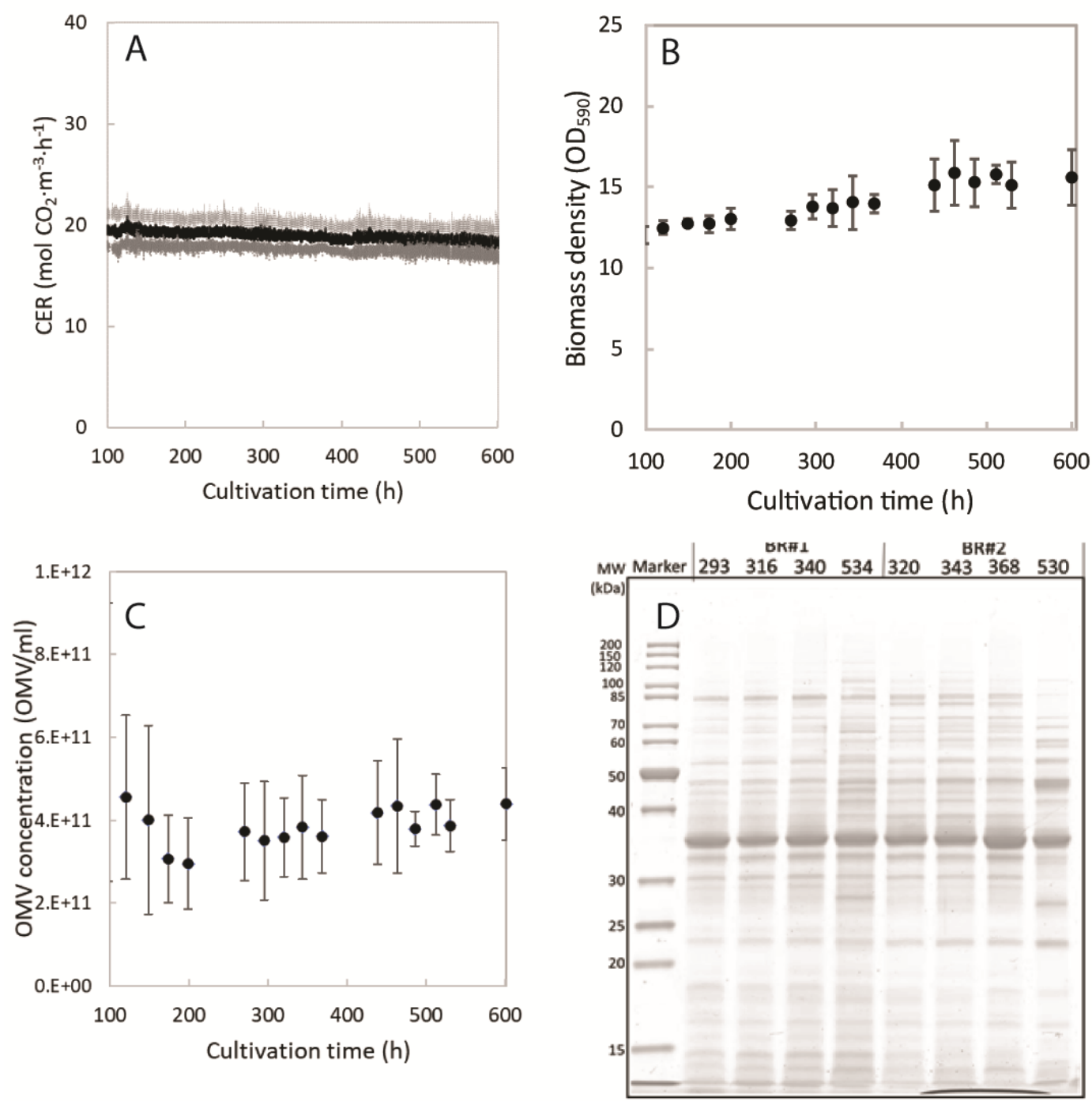

Figure 3. Reproducibility of continuous OMV production in Nm chemostat cultures. 5 chemostat cultures were compared to assess the culture to culture variation in OMV productivity. Graph A shows the mean carbon dioxide evolution rate (CER, black line) and standard deviation (grey line). Graph B shows the average biomass density and Graph C shows the OMV concentration. Error bars indicate the standard deviation from the mean. The protein composition is shown of OMVs purified from two replicates of a steady state chemostat culture at different times in the cultivation (Graph D).

\section{Optimization of the continuous sOMV production}

The dilution rate is a critical process parameter of continuous production processes. The continuous cultures described here so far, were based on a practical dilution rate of $1 /$ day $\left(0.04 \mathrm{~h}^{-1}\right)$. To optimize the volumetric productivity, an accelerostat culture was performed by slowly increasing the dilution rate $\left(\alpha_{D}=0.0055 \mathrm{~h}^{-2}\right)$ of a steady state chemostat culture. Previously, using the same accelerostat culture, we already showed 
that the specific productivity of $\mathrm{Nm}$ was only minorly influenced by the growth rate between $0.03 \mathrm{~h}^{-1}$ and $0.16 \mathrm{~h}^{-1}$ [33]. Since the specific productivity remains constant, the volumetric productivity increases linearly with the dilution rate (Figure 4). Thus, operating the continuous culture at $0.15 \mathrm{~h}^{-1}$ shows the highest volumetric productivity of $1.0 \times 10^{15}$ OMVs per liter reactor volume per day.

Next, a chemostat was run at 3.6/day $\left(0.15 \mathrm{~h}^{-1}\right)$ to confirm the results from the accelerostat culture. For this, the dilution rate of a steady state culture at $0.04 \mathrm{~h}^{-1}$ was increased to $0.15 \mathrm{~h}^{-1}$ (Data not shown). This steady state at $0.15 \mathrm{~h}^{-1}$ showed an OMV concentration of $2.1 \times 10^{14} \mathrm{OMVs} / \mathrm{L}$, which was slightly lower than the OMV concentration of $2.5 \times 10^{14} \mathrm{OMVs} / \mathrm{L}$ in the accelerostat culture at this dilution rate. As a result, the volumetric productivity was $7.4 \times 10^{14} \mathrm{OMVs} / \mathrm{L} /$ day, which is slightly lower than the expected volumetric productivity of $1.0 \times 10^{15} \mathrm{OMVs} / \mathrm{L} /$ day based on the accelerostat data. Taken together, a dilution rate of $0.15 \mathrm{~h}^{-1}$ shows an increased volumetric productivity of compared to the volumetric productivity of $4.0 \times 10^{14}$ $\mathrm{OMV} / \mathrm{L} /$ day at a dilution rate of $0.04 \mathrm{~h}^{-1}$.

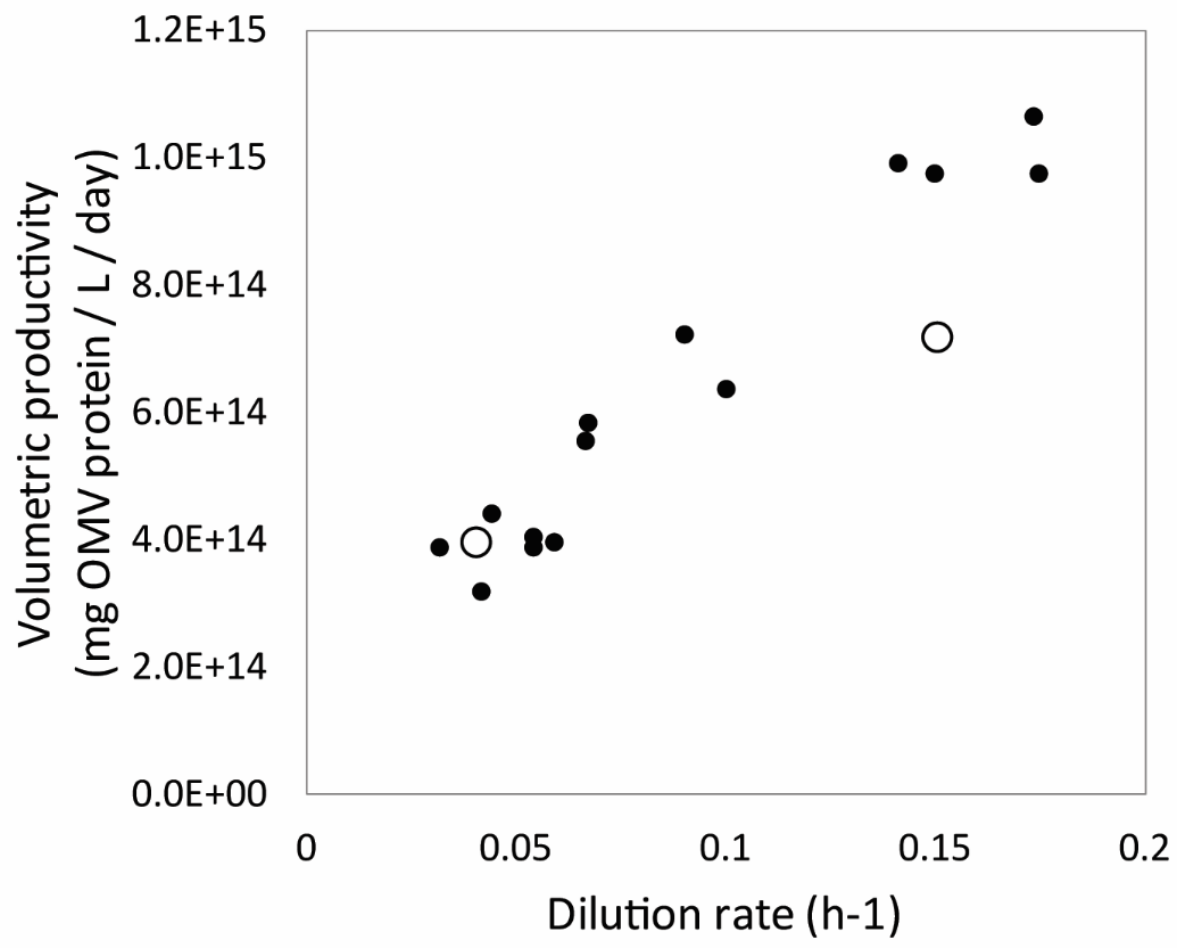

Figure 4. Volumetric OMV productivity as a function of the dilution rate. The volumetric productivity of SOMVs in an accelerostat culture of $\mathrm{Nm}$ shows to be linearly related to the dilution rate for growth rates between $0.03 \mathrm{~h}^{-1}$ and $0.18 \mathrm{~h}^{-1}$ (solid circles) The volumetric productivities of steady-state cultures at $0.04 \mathrm{~h}^{-1}$ and $0.15 \mathrm{~h}^{-1}$ are depicted as open circles. 


\section{Comparison to batch and fed-batch production}

The production of OMVs in continuous mode was compared to the production in batch and fed-batch mode. We compared these cultures (data shown in Supplemental Figure 3 ) to the continuous production of OMVs (Table 1). For this, we assumed that 20 days of harvest could be obtained from a continuous bioreactor per month, while for the batch process one harvest can be obtained per week. For the fed-batch production mode, we assumed an identical throughput time of one week as for the batch process since the fed-batch process requires only 12 hours more cultivation time than the batch process. Additionally, we assumed similar recoveries of OMVs in downstream purification regardless of the OMV production method. Based on these assumptions, 16-fold more volume could be processed from a continuous reactor on a yearly basis with a dilution rate of $0.15 \mathrm{~h}^{-1}$ (Table 1). This corresponds to an 9-fold increase in OMV production in this continuous mode compared to batch mode. DNA release during the steady state of continuous cultivations was significantly reduced compared to the batch process.

During batch and fed-batch cultivations, significant amounts of DNA accumulate caused by lysis of the bacteria (Supplemental Figure 3). Furthermore, accumulation of ammonium was observed during the glucose-limited fed-batch cultures, which inhibits enzymatic DNA degradation during OMV purification. Continuous OMV production is thus an improved method over batch and fed-batch production. 
Table 1. Production characteristics of batch, fed-batch, and continuous OMV production.

Benchtop N. meningitidis cultures were operated in three different operating modes. Batch mode is characterized by triggered OMV release through cysteine depletion. In fed-batch mode, glucose and glutamate was added to the culture to prolong the OMV releasing state. The continuous culture represents the steady state values of a culture with a dilution rate of $1 /$ day. Values represent the mean and standard deviation of triplicate cultures, except for the continuous culture at the dilution rate of 3.6/day that represents a single replicate. Non-colored cells indicate measured values, grey cells indicate assumptions, and light green indicate calculated values.

\begin{tabular}{|c|c|c|c|c|}
\hline & BATCH & FED-BATCH & CONTINUOUS & CONTINUOUS \\
\hline CULTIVATION TIME (H) & 24 & 40 & 1 volume / $24 \mathrm{~h}$ & 3.6 volumes / $24 \mathrm{~h}$ \\
\hline$[\mathrm{OMV}]\left(10^{13} / \mathrm{L}\right)$ & $38 \pm 6$ & $52+9$ & $40 \pm 7$ & 21 \\
\hline $\begin{array}{l}\text { BIOMASS DENSITY } \\
\left(O D_{590}\right)\end{array}$ & $8.7 \pm 1.5$ & $11.1 \pm 0.3$ & $14 \pm 1.6$ & - \\
\hline [DNA] (MG/L) & $1.3 \pm 0.2$ & $0.48 \pm 0.02$ & $0.09 \pm 0.03$ & - \\
\hline [AMMONIUM] (MM) & $46 \pm 2$ & $51 \pm 3$ & $34 \pm 5$ & - \\
\hline $\begin{array}{l}\text { VOLUMETRIC } \\
\text { PRODUCTIVITY (1014 } \\
\text { OMV/L/DAY) }\end{array}$ & 3.8 & 5.2 & 4.0 & 7.4 \\
\hline $\begin{array}{l}\text { HARVEST VOLUMES } \\
\text { PER CAMPAIGN }\end{array}$ & 1 & 1 & 20 & 72 \\
\hline CAMPAIGNS / YEAR & 52 & 52 & 12 & 12 \\
\hline $\begin{array}{l}\text { YEARLY } \\
\text { PRODUCTIVITY (10 }{ }^{13} / \mathrm{L} \\
\text { BIOREACTOR VOLUME) }\end{array}$ & 1976 & 2704 & 9600 & 17712 \\
\hline
\end{tabular}

\section{Discussion}

Continuous cultivations of $\mathrm{Nm}$ at a dilution rate of 1/day showed high and reproducible OMV concentrations. Increased volumetric productivities could be obtained, compared to batch and fed-batch cultivations. Further intensification of the process is possible by optimizing the volumetric productivity by increasing the dilution rate. Continuous OMV production at a dilution rate of 3.6/day results in a 9-fold increase in OMV production compared to batch-wise OMV production. 
The start of the continuous cultivation at a dilution rate of 1 /day showed an adjustment period of multiple dilutions, possibly caused by the complexity of the medium. The steady state was characterized by depletion of both carbon sources glucose and glutamate, as well as cysteine and arginine. Addition of glucose to a steady state culture resulted in bacterial growth, indicating that the culture was glucose limited. Future medium optimization should reduce the complexity of the medium, resulting in a single nutrient limitation that possibly reduces the time required to reach a steady state. Additionally, steady state biomass productivity can be optimized by future medium optimization. Specific OMV productivities can be further enhanced by inducing OMV release by for example high dissolved oxygen concentrations [17] .

Increased dilution rates were assessed in an accelerostat culture. At dilution rates above $0.18 \mathrm{~h}^{-1}$, the accelerostat culture showed reduced biomass concentrations, and was stopped. The maximum specific growth rate of $\mathrm{Nm}$ in batch cultures on this medium is $0.5 \mathrm{~h}^{-1}$. This difference is possibly caused by a different nutrient consumption profile at these different growth rates. Additionally, the release of OMVs during exponential growth in batch cultures is low [18]. Dilution rates of $0.18 \mathrm{~h}^{-1}$ and above are thus not expected to further improve the volumetric productivity due to an anticipated decrease in specific productivity. Therefore, the volumetric OMV productivity on this medium composition is maximized at dilution rates just below $0.18 \mathrm{~h}^{-1}$. To compare the continuous OMV production with batch and fed-batch OMV production, we used the dilution rate of $0.15 \mathrm{~h}^{-1}$ (3.6/day) and assumed this state can be maintained for 20 days. This length should be carefully considered based on future research to the stability of $\mathrm{Nm}$ upon prolonged cultivations. The genome of $\mathrm{Nm}$ is generally known as variable [34], although Nm subcultured after a production cultures showed to be genetically stable for at least 30 generations during exponential growth [14]. Besides monitoring of the genetically stability of the production culture, the OMV product should be characterized completely and monitored throughout production cultures. Here we looked at protein profile and OMV size and found no significant changes over the course of the continuous cultivation, indicating the feasibility of the process. In comparison, perfusion based monoclonal antibody production systems have been described to be able of production periods of over 60 days [35]. The advantage of perfusion systems is that the growth rate can be kept low resulting in less bacterial generations in time. 
Here we showed the continuous upstream production of OMVs. Purification of OMVs should be designed in a continuous manner to obtain a fully continuous OMV production process. Continuous separation of OMVs from the biomass, while maintaining the bacterial culture in a steady state, would be the most straightforward initial separation. Tangential flow microfiltration directly on the continuous reactor could be used. Such an approach would have similarities to high cell density perfusion based cultivation systems [36, 37]. Interestingly, a Nm perfusion based cultivation system has been described [38]. This system enabled the biomass production of 58 gram dry weight per L, although the amount of OMVs secreted in the culture was not described [38].

The development of new technologies for cleaner and more efficient manufacturing are supported by regulatory authorities [22], although currently no continuous production processes of vaccine have been described. Important aspects as suitable inline process analytical tools and the possibility of mutations introduced during the continuous production have to be addressed before registration of continuous products will be in sight. Continuous biopharmaceutical production processes have been researched for therapeutic proteins [39] and small molecules [40]. Recently, two production processes of monoclonal antibodies in a fully continuous manner have been described $[41,42]$.

This study shows the potential of continuous production of Nm OMVs to reach high volumetric OMV productivities. The high OMV productivities could be beneficial for the production of low-cost biotechnological applications based on OMVs such as enzyme carriers. Future development should focus on a fully continuous purification processes. Further research on online product quality analysis methods and batch-to batch variability could be the basis of future continuous OMV production process for OMV adjuvants and low-cost price OMV-based vaccines.

\section{Acknowledgements}

The authors thank Robert van de Put and Alex de Haan for the metabolite measurements and the Process Development Bacterial Vaccines team for their help in performing lengthy continuous cultivations. This work has been funded by the Ministry for Health, Welfare and Sports (The Netherlands). 


\section{References}

1. Kuehn, M.J. and N.C. Kesty, Bacterial outer membrane vesicles and the host-pathogen interaction. Genes \& Development, 2005. 19(22): p. 2645-2655.

2. Kulp, A. and M.J. Kuehn, Biological Functions and Biogenesis of Secreted Bacterial Outer Membrane Vesicles. Annual Review of Microbiology, 2010. 64(1): p. 163-184.

3. Park, M., et al., Positional Assembly of Enzymes on Bacterial Outer Membrane Vesicles for Cascade Reactions. PLOS ONE, 2014. 9(5): p. e97103.

4. Wang, S., J. Gao, and Z. Wang, Outer membrane vesicles for vaccination and targeted drug delivery. Wiley Interdisciplinary Reviews: Nanomedicine and Nanobiotechnology, 2018. 0(0): p. e1523.

5. Acevedo, R., et al., Bacterial Outer Membrane Vesicles and Vaccine Applications. Frontiers in Immunology, 2014. 5(121).

6. Tan, K., et al., Outer Membrane Vesicles: Current Status and Future Direction of These Novel Vaccine Adjuvants. Frontiers in Microbiology, 2018. 9: p. 783.

7. Granoff, D.M., Review of Meningococcal Group B Vaccines. Clinical Infectious Diseases, 2010. 50(Supplement_2): p. S54-S65.

8. Panatto, D., et al., Neisseria meningitidis B vaccines. Expert Review of Vaccines, 2011. 10(9): p. 1337 1351.

9. Sierra, G.V., et al., Vaccine against group B Neisseria meningitidis: protection trial and mass vaccination results in Cuba. NIPH annals, 1991. 14(2): p. 195-207; discussion 208-10.

10. Cassio de Moraes, J., et al., Protective efficacy of a serogroup B meningococcal vaccine in Sao Paulo, Brazil. The Lancet, 1992. 340(8827): p. 1074-1078.

11. Bjune, G., et al., Effect of outer membrane vesicle vaccine against group B meningococcal disease in Norway. The Lancet, 1991. 338(8775): p. 1093-1096.

12. Serruto, D., et al., The new multicomponent vaccine against meningococcal serogroup $B, 4 C M e n B$ : Immunological, functional and structural characterization of the antigens. Vaccine, 2012. 30: p. B87-B97.

13. van der Ley, P. and G. van den Dobbelsteen, Next-generation outer membrane vesicle vaccines against Neisseria meningitidis based on nontoxic LPS mutants. Human Vaccines, 2011. 7(8): p. 886-890.

14. van de Waterbeemd, B., et al., Improved OMV vaccine against Neisseria meningitidis using genetically engineered strains and a detergent-free purification process. Vaccine, 2010. 28(30): p. 4810-6.

15. Gerritzen, M.J.H., et al., Spontaneously released Neisseria meningitidis outer membrane vesicles as vaccine platform. Submitted_for_publication.

16. Gerritzen, M.J.H., et al., Insights in sulfur depletion triggered outer membrane vesicle production by Neisseria meningitidis. in preparation.

17. Gerritzen, M.J.H., et al., High dissolved oxygen tension triggers outer membrane vesicle formation by Neisseria meningitidis. Microbial Cell Factories, 2018. 17(1): p. 157.

18. van de Waterbeemd, B., et al., Cysteine depletion causes oxidative stress and triggers outer membrane vesicle release by Neisseria meningitidis; implications for vaccine development. PLoS One, 2013. 8(1): p. e54314.

19. Pollock, J., et al., Integrated continuous bioprocessing: Economic, operational, and environmental feasibility for clinical and commercial antibody manufacture. Biotechnology Progress, 2017. 33(4): p. 854-866.

20. Konstantinov, K.B. and C.L. Cooney, White Paper on Continuous Bioprocessing May 20-21 2014 Continuous Manufacturing Symposium. Journal of Pharmaceutical Sciences, 2015. 104(3): p. 813-820.

21. Lee, S.L., et al., Modernizing Pharmaceutical Manufacturing: from Batch to Continuous Production. Journal of Pharmaceutical Innovation, 2015. 10(3): p. 191-199.

22. Allison, G., et al., Regulatory and Quality Considerations for Continuous Manufacturing May 20-21, 2014 Continuous Manufacturing Symposium. Journal of Pharmaceutical Sciences, 2015. 104(3): p. 803-812.

23. Holten, E., Serotypes of Neisseria meningitidis isolated from patients in Norway during the first six months of 1978. J Clin Microbiol, 1979. 9(2): p. 186-8.

24. Gerritzen, M.J.H., et al., High throughput nanoparticle tracking analysis for monitoring outer membrane vesicle production. Journal of Extracellular Vesicles, 2017. 6(1): p. 1333883.

25. van der Ley, P., et al., Modification of lipid A biosynthesis in Neisseria meningitidis lpxL mutants: influence on lipopolysaccharide structure, toxicity, and adjuvant activity. Infect Immun, 2001. 69(10): p. 5981-90.

26. Tommassen, J., et al., Isolation of Neisseria meningitidis mutants deficient in class 1 (porA) and class 3 (porB) outer membrane proteins. Infect Immun, 1990. 58(5): p. 1355-9.

27. Steeghs, L., et al., Neisseria meningitidis expressing lgtB lipopolysaccharide targets DC-SIGN and modulates dendritic cell function. Cell Microbiol, 2006. 8(2): p. 316-25. 
28. Baart, G.J.E., et al., Scale-up for bulk production of vaccine against meningococcal disease. Vaccine, 2007. 25(34): p. 6399-6408.

29. Malloy, A. and B. Carr, NanoParticle Tracking Analysis - The Halo ${ }^{\text {TM }}$ System. Particle \& Particle Systems Characterization, 2006. 23(2): p. 197-204.

30. Dorresteijn, R.C., et al., Determination of amino acids using o-phthalaldehyde-2-mercaptoethanol derivatization effect of reaction conditions. Journal of Chromatography A, 1996. 724(1): p. 159-167.

31. Baart, G.J., et al., Modeling Neisseria meningitidis metabolism: from genome to metabolic fluxes. Genome Biol, 2007. 8(7): p. R136.

32. Gerritzen, M.J.H., et al., Sulfate depletion triggers overproduction of phospholipids and the release of outer membrane vesicles by Neisseria meningitidis Submitted_for_publication.

33. Gerritzen, M.J.H., et al., High dissolved oxygen tension triggers outer membrane vesicle formation by Neisseria meningitidis. Manuscript submitted for publication, 2018.

34. Schoen, C., et al., Genome flexibility in Neisseria meningitidis. Vaccine, 2009. 27(Suppl 2): p. B103-B111.

35. Whitaker, S., R. Francis, and R. Siegel, Validation of continuously perfused cell culture processes for production of monoclonal antibodies. 1998.

36. Clincke, M.-F., et al., Very high density of CHO cells in perfusion by ATF or TFF in WAVE bioreactor ${ }^{T M}$. Part I. Effect of the cell density on the process. Biotechnology Progress, 2013. 29(3): p. 754-767.

37. $\mathrm{Xu}, \mathrm{S}$. and $\mathrm{H}$. Chen, High-density mammalian cell cultures in stirred-tank bioreactor without external $\mathrm{pH}$ control. Journal of Biotechnology, 2016. 231: p. 149-159.

38. Dehottay, P.M.H., et al., Fermentation of fastidious bacterial strain in perfusion suspension culture. 2014, GLAXOSMITHKLINE BIOLOGICALS S.A.

39. Karst, D.J., F. Steinebach, and M. Morbidelli, Continuous integrated manufacturing of therapeutic proteins. Current Opinion in Biotechnology, 2018. 53: p. 76-84.

40. Kleinebudde, P., J. Khinast, and J. Rantanen, Continuous manufacturing of pharmaceuticals. Vol. 7703. 2017: John Wiley \& Sons.

41. Godawat, R., et al., End-to-end integrated fully continuous production of recombinant monoclonal antibodies. Journal of Biotechnology, 2015. 213: p. 13-19.

42. Karst, D.J., et al., Process performance and product quality in an integrated continuous antibody production process. Biotechnology and Bioengineering, 2016. 114(2): p. 298-307. 


\section{Supplementary data}
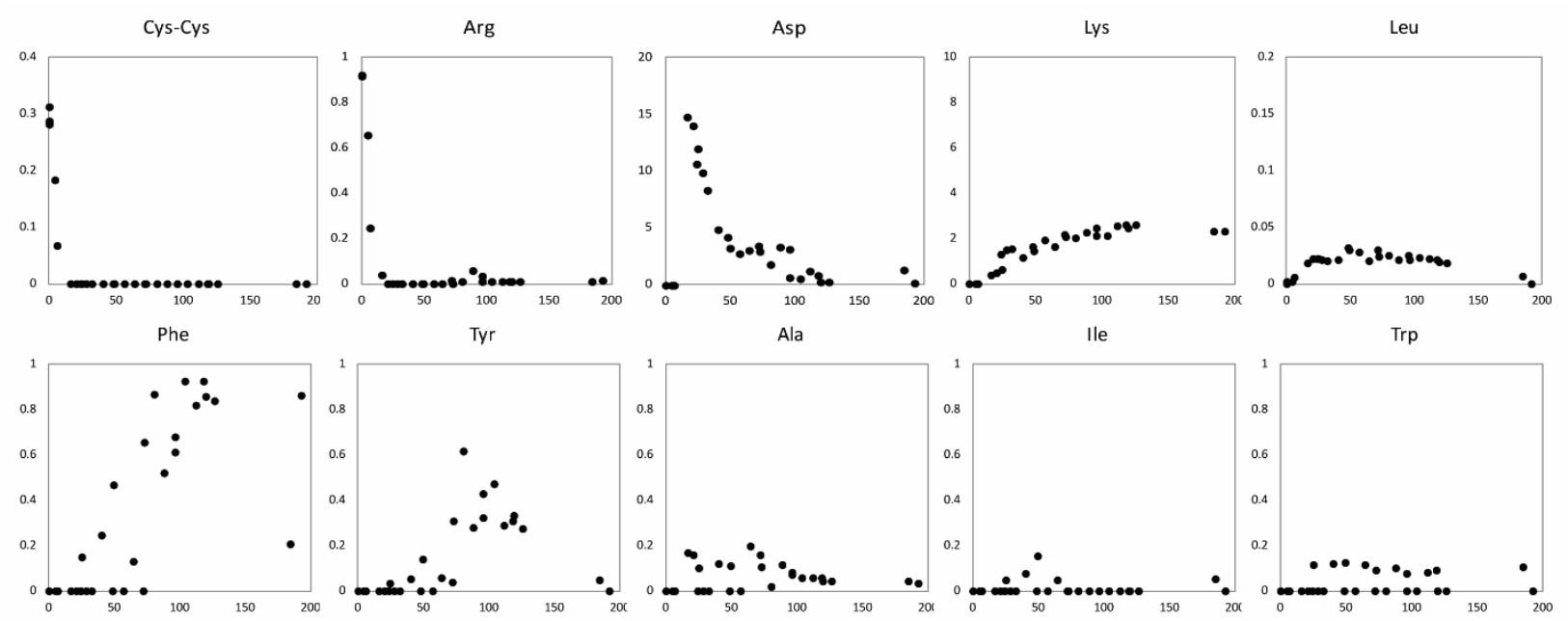

Ala

Ile

Trp
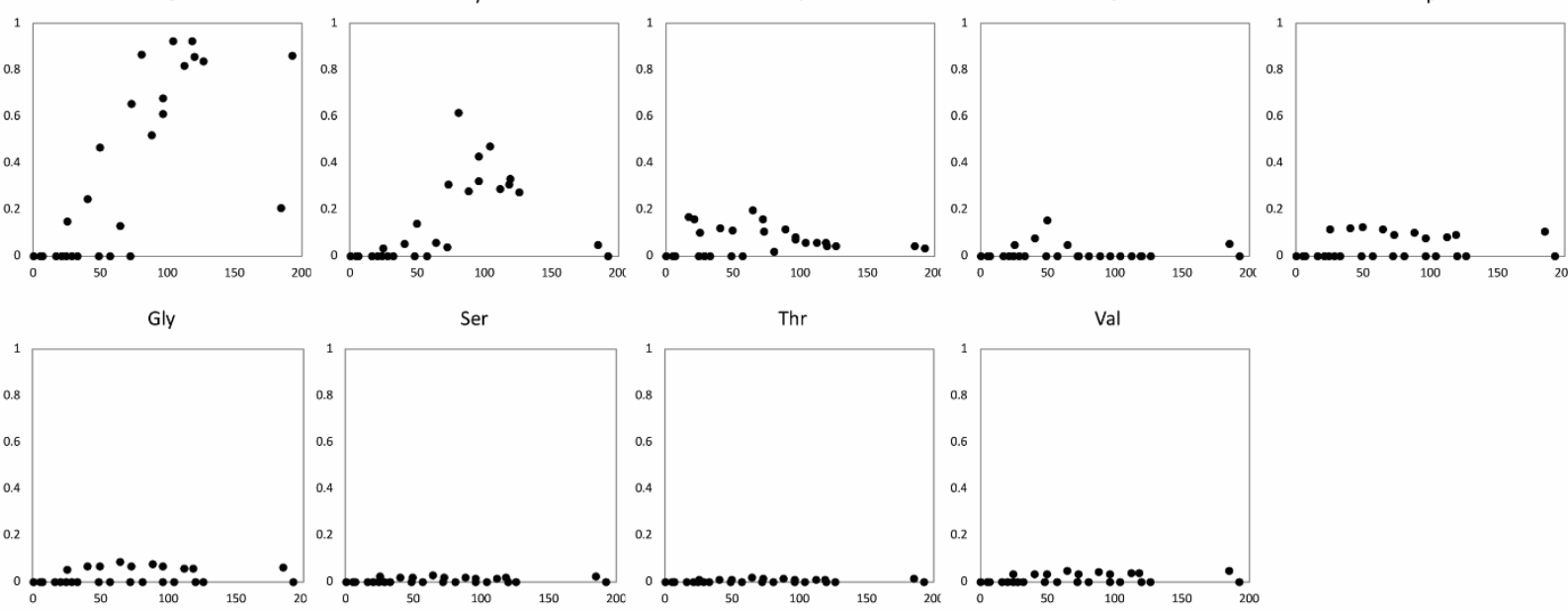

Thr

Val
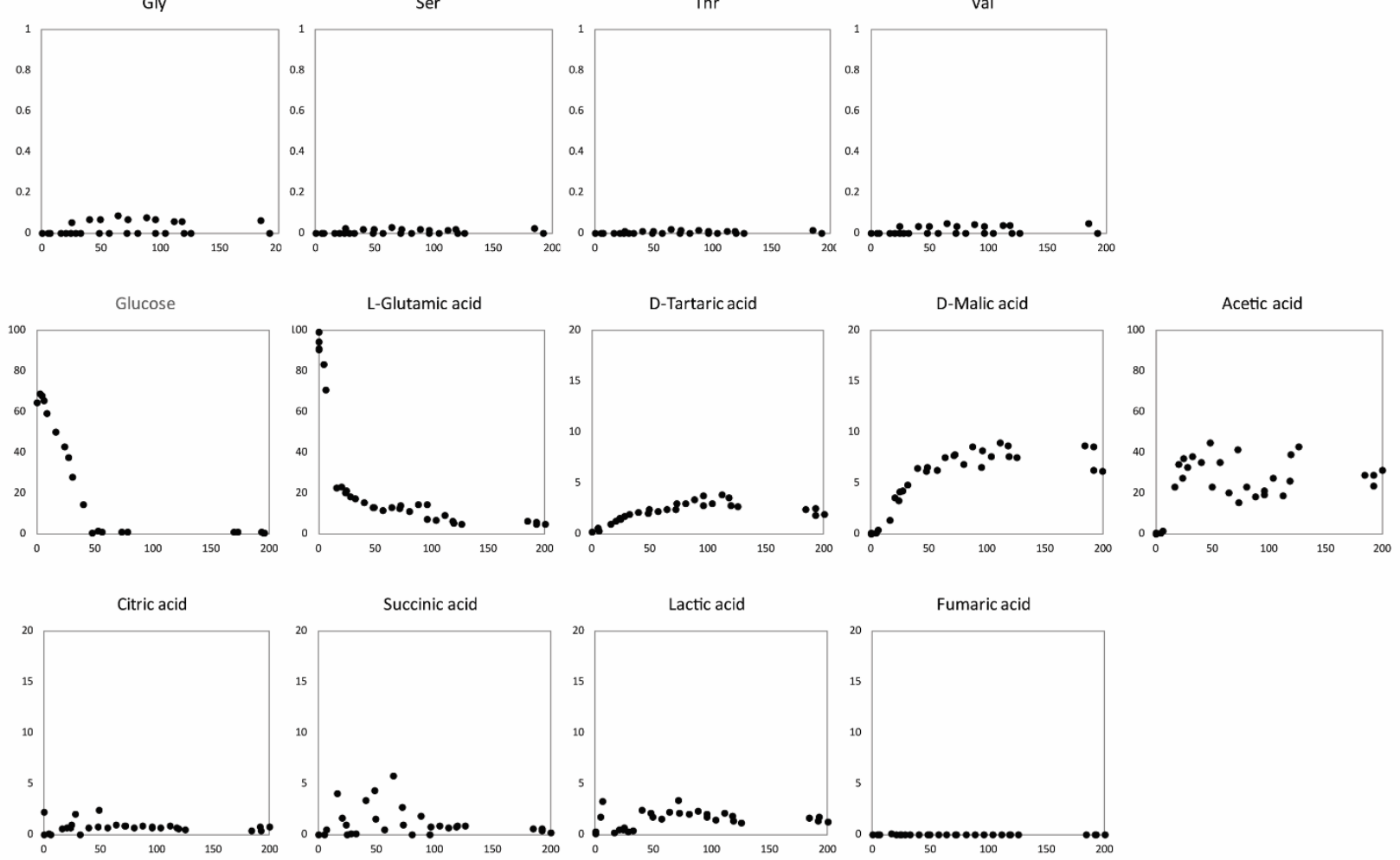

Supplemental Figure 1. Nutrient and metabolite profile of the chemostat. Measurements represent

the overlay of samples of the adaptation phased from two replicate chemostat cultures of $\mathrm{Nm}$ with a dilution rate of 1 day. All concentration measurement are in mM. Amino acids cystine (Cys-cys), arginine (arg), aspartate (Asp), lysine (Lys), leucine (Leu), phenylalanine (Phe), tyrosine (Tyr), alanine (Ala), isoleucine (Ile), tryptophan (Trp), glycine (Gly), serine (Ser) , threonine (Thr), and valine (Val) are measured, asparagine, glutamine, histidine, methionine, hydroxyproline, and proline were not detected (data not shown). 
A

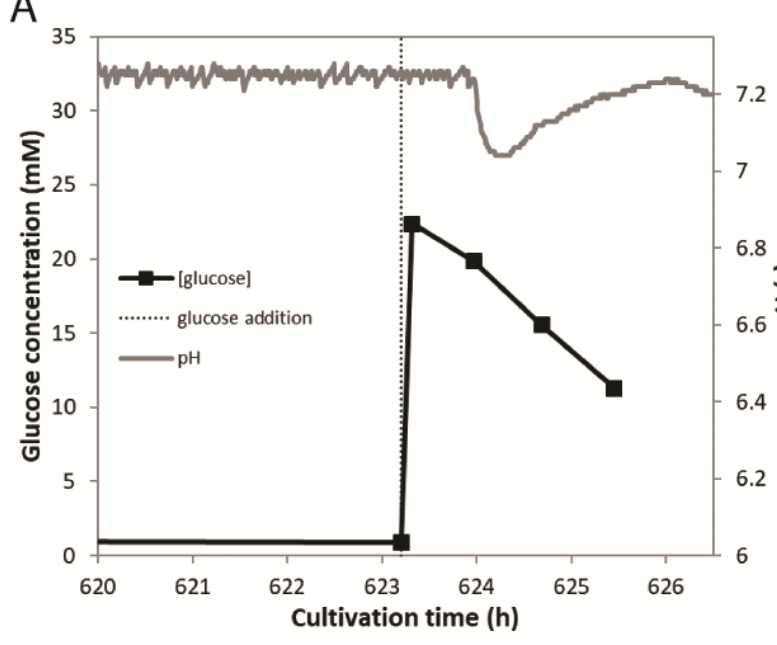

B

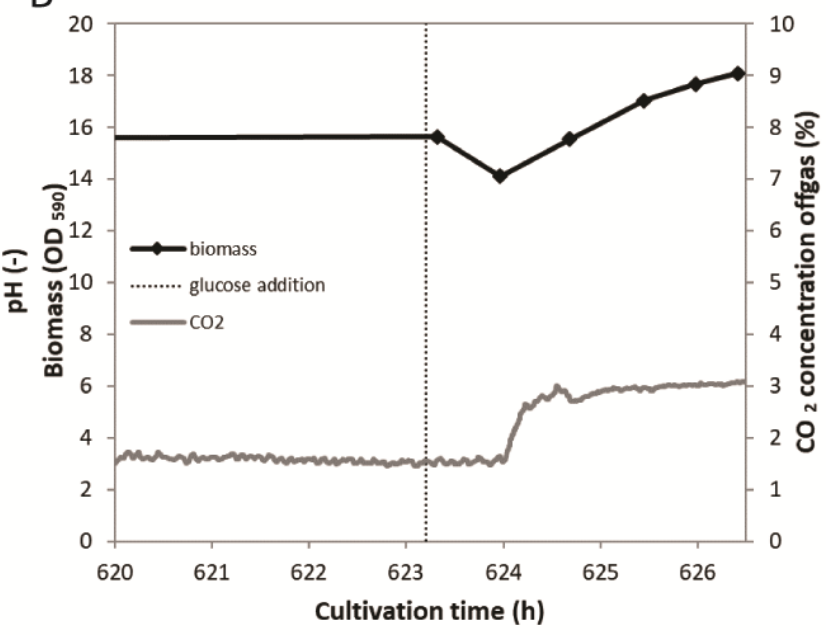

Supplemental Figure 2. Identification of the limiting nutrient. To a steady state culture of $N$. meningitidis, a shot of glucose solution was added to increase the glucose concentration with $20 \mathrm{mM}$ (graph A, dashed line). The biomass concentration is measured by optical density measurements and the carbon dioxide concentration was measured in the offgas (graph B).
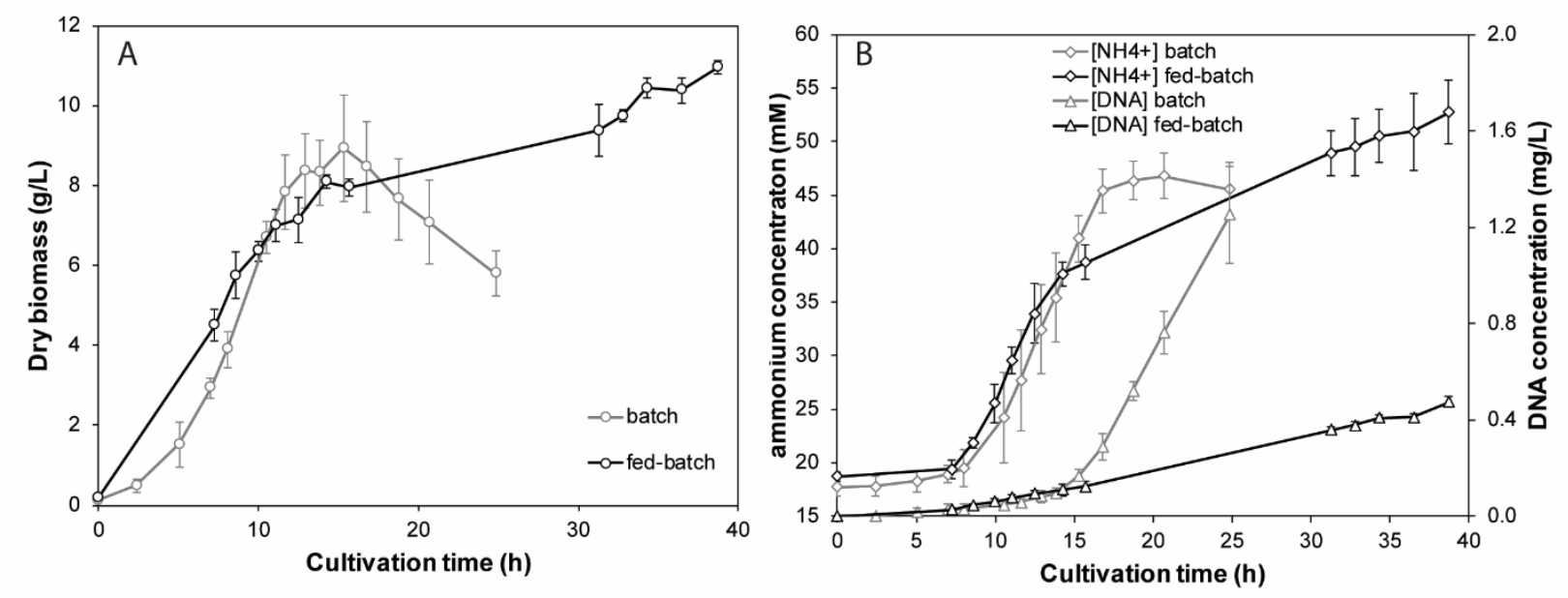

Supplemental Figure 3. Batch and fed-batch $\boldsymbol{N}$. meningitidis cultures. $\mathrm{Nm}$ cultures were operated in batch-mode, as well as in fed-batch mode using a glucose feed solution (Graph A). Accumulation of ammonia and DNA was measured in both cultures (Graph B). Data represents mean value of triplicate cultures; error bars indicate the standard deviation. 
Chapter 8

General discussion 


\section{Vaccine platforms}

Infectious diseases spread quickly in today's global and interconnected world. In the 21st century there have been outbreaks of new emerging diseases like Zika, Ebola, and SARS, as well as outbreaks of known diseases like cholera, plaque, and yellow fever [2]. The threat of both new emerging diseases as well as outbreaks of old diseases can be mitigated by development of new vaccines. For example, meningococcal serogroup $\mathrm{W}$ infections have risen recently in The Netherlands. As a response, The Dutch National Immunization Programme introduced meningococcal serogroup $\mathrm{W}$ vaccination by expanding the meningococcal vaccination from the single serogroup $\mathrm{C}$ vaccine to $\mathrm{a}$ combinatorial serogroup A, C, W, Y vaccine $[3,4]$, which was already available. Additionally, there is need for the development of new vaccines for bacterial infectious diseases, that were traditionally treated by antibiotic drugs [5]. More and more bacterial strains are acquiring antibiotic resistance and pathogens emerge that are virtually resistant against all available antibiotics [6]. The development of new prophylactic vaccines can be a solution for these multidrug resistant pathogens [7]. Moreover, the development of new therapeutic vaccines requires the development of vaccines against many new targets.

The vaccine development trajectory is a lengthy process for each new target. Traditional vaccines are based on the inactivation of the pathogens or on live attenuated pathogens. For their production, large amounts of pathogens are required, which form a risk to the production workers and to the environment in the case of impaired biocontainment [8]. The use of a vaccine platform, where the new vaccine antigens can be added or exchanged, can shorten the development period for new vaccines. This modular vaccine approach requires the identification of antigens, that can subsequently be expressed on the platform base (Figure 1). The immunogenicity of the expressed antigen should be enhanced by expressing it on the vaccine platform base, such that a safe vaccine is obtained without the risks associated to the pathogen to which the vaccine should induce protection. Additionally, the vaccine platform base should result in proper stability and allow for detailed characterization of the modular vaccine. A vaccine platform requires thorough initial development of the platform to ensure the robustness of the platform. This includes the development of a high yielding production process. Once the platform is developed, new vaccines can be generated in a much shorter time. Subunit vaccines, which are purified antigens produced in microbes, are 
an example but do require proper adjuvating agents to induce a proper immune response. Expression of antigens on OMVs, however, can be a platform that is selfadjuvating due to the OMV composition, and thus possibly an improved basis for vaccine platform development. In this chapter, the use of OMVs as vaccine platform is discussed with a focus on the production of OMVs and an outlook is given on future OMV-based vaccines for serogroup B meningococcal disease and other bacterial infection diseases.

\section{OMVs as vaccine platform}

Bacterial outer membrane vesicles (OMVs) are a good candidate for such a vaccine platform. OMVs are non-replicating nanoparticles that are packed with antigens and are self-adjuvating. OMVs contain proteins, phospholipids, and lipopolysaccharides derived from the bacterial outer membrane [9]. Outer membrane vesicles have been used in multiple meningococcal vaccines that were successfully used to stop epidemics in different countries [10-15]. The use of OMVs as vaccines has thus been shown to be safe and effective. A vaccine platform based on OMVs requires the addition of heterologous antigens to OMVs. Heterologous antigens can be added by expressing them in the OMV producing bacterium or by addition to the OMV by chemical coupling. The production process developed in this thesis is for a vaccine platform based on OMVs derived from

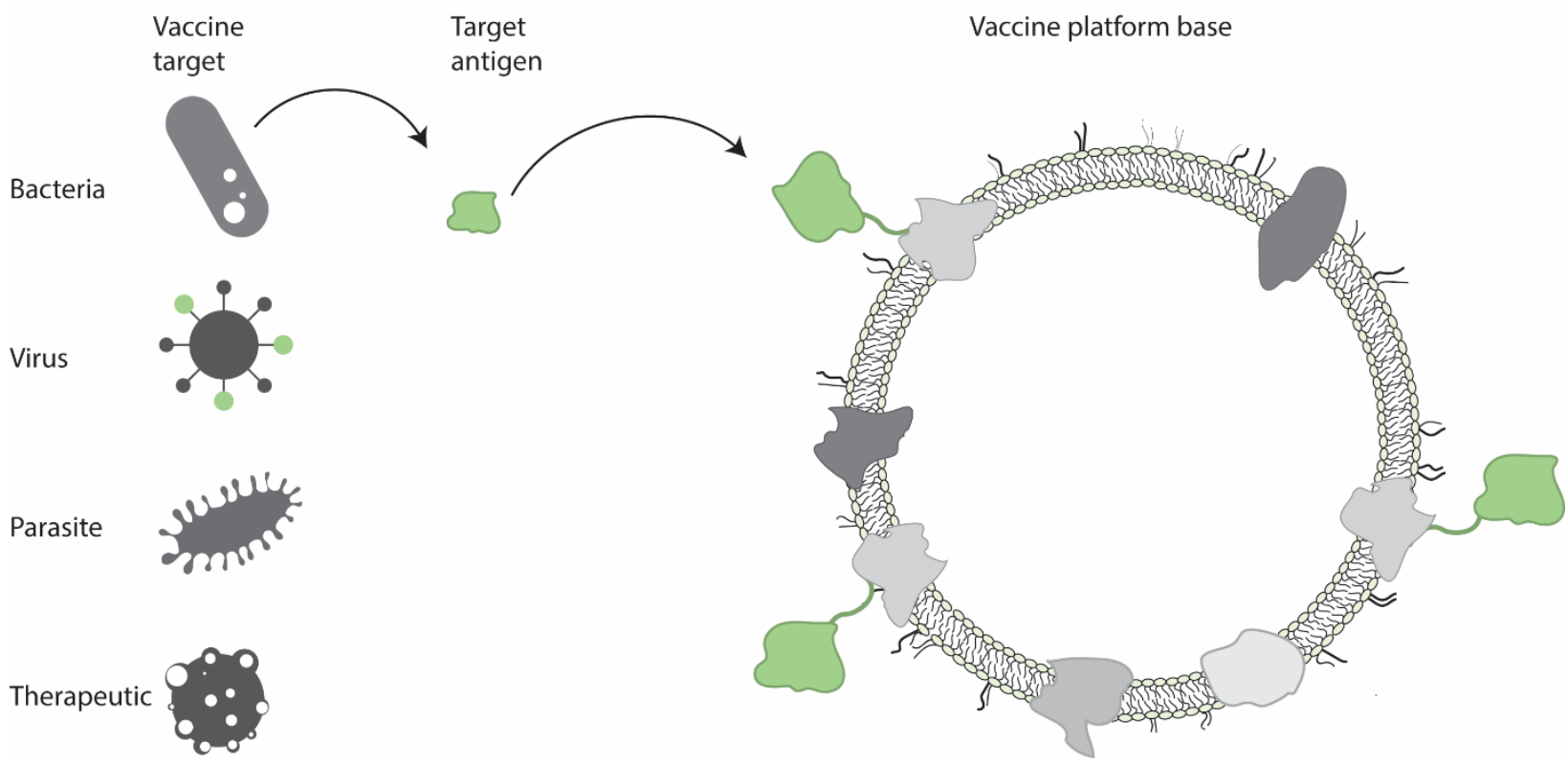

Figure 1. Modular vaccine approach. The basis of a vaccine platform is the addition of antigens of a vaccine target to a vaccine platform base. The vaccine platform base is here a bacterial outer membrane vesicle, that is a non-replicative nanoparticle derived from the outer membrane of the bacterium. 
Neisseria meningitidis ( $\mathrm{Nm}$ ) where the heterologous antigens are expressed by the bacterium itself. Nm OMVs are suitable as modular base for both prophylactic and therapeutic vaccines.

The process development of most Nm OMV vaccine production processes have been based on detergent extraction (dOMV) to reduce the content of endotoxic LPS in Nm OMVs. This results in OMVs with reduced LPS and phospholipid content compared to biologically derived OMVs [1] (Figure 2). This reduction in LPS was required to prevent endotoxic effects. However, genetically modified LPS variants have been developed that result in reduced endotoxic effects, while maintaining its potency to stimulate the immune response [16]. This opens the possibility to use sOMVs as a basis for a vaccine platform. In addition, OMV formation of both extracted OMVs and spontaneously released OMVs can be increased by reducing the linkage of the outer membrane to the peptidoglycan layer. In $\mathrm{Nm}$, the knockout of the peptidoglycan binding periplasmic protein RmpM, showed 2-fold increases in dOMV production and 10-fold increases in OMV released spontaneously by the bacterium (sOMV) [1]. The production of Nm OMVs by purification of OMVs released by the bacteria in the culture supernatant is advantageous over extraction-based OMV production. Less process steps are required as the extraction of OMVs can be omitted making the OMV production process more straightforward and more cost-effective. Therefore, OMVs released in the supernatant of the bacterial culture were chosen as the basis of the OMV vaccine platform.
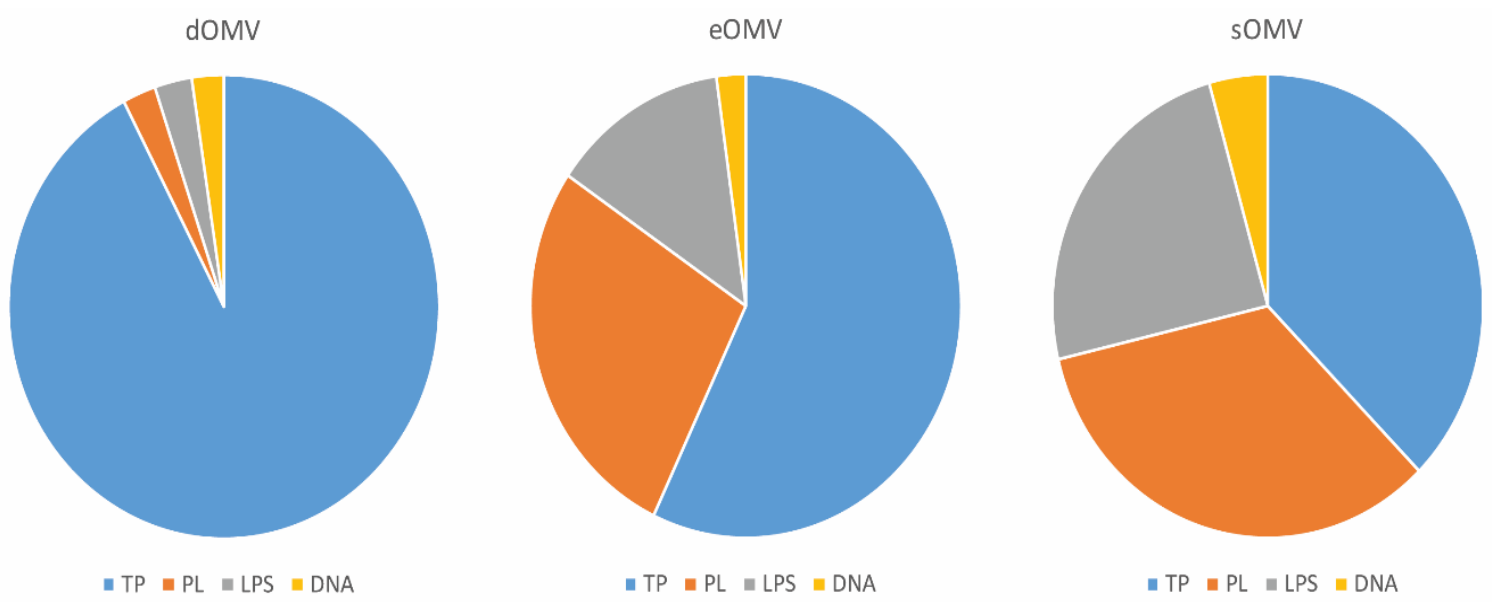

Figure 2. Biochemical composition of Nm OMVs. OMVs are extracted by detergent (dOMVs), EDTA (eOMV), or spontaneously released (sOMV). The composition of the major biochemical components was analyzed and given in relative amounts (weight \%) by total protein (TP), phospholipid (PL), lipopolysaccharide (LPS), and DNA. The presence of large residual amounts of detergent in dOMV or EDTA in eOMV are not measured and thus not included in this graph. Data adapted from Van de Waterbeemd et al. [1]. 
The platform was based on Nm due to the known safety of Nm OMVs [17] and the ability to express heterologous antigens in $\mathrm{Nm}$. The use of a pathogenic bacterium for the platform was preferred over lab-strains as E. coli, to exclude undesired immune responses against commensal bacteria. Additionally, immune responses directed against the OMV backbone, that contains components of the OMV producing microbe, could reduce the efficacy of the vaccine by reducing the immune responses against the heterologous antigen or by reduced immune responses upon repetitive use. Therefore, we reduced the antigenic content of the Nm OMVs by removing the major antigenic outer membrane porin PorA. Nevertheless, more research is required on the effect of immune responses against the OMV backbone. Despite all the research performed previously on SOMV biogenesis, the yield of Nm SOMV production processes is still low. To improve the yield of sOMV production, either the number of OMVs produced per bacterium can be targeted or the amount of biomass generated in the production process. In this thesis, we focused on increasing the amount of sOMVs released per bacterium by increasing our understanding on OMV release.

\section{Production of OMVs}

To study OMV production, a new method for direct OMV quantification was required. The first OMVs were observed as outer membrane fragments by radioactive labelling and detection in the cell free supernatant of bacterial cultures [18]. With electron microscopy it could be observed that the outer membrane fragments were spherical nanoparticles [19]. Additionally, OMV quantification can be based on protein or phospholipid content after purification of OMVs from the sample by ultracentrifugation or filtration. Electron microscopy is not a quantitative method and both electron microscopy and phospholipid analysis are elaborate and time-consuming. Real-time quantification of OMVs would accelerate process development. New techniques can be used for direct detection of nanoparticles, for example tunable resistance pulse sensing (TRPS), nanoparticle tracking analysis (NTA), or size exclusion chromatography-multi angle light scattering (SEC-MALS) [20]. In chapter 3, we showed that NTA can be applied to measure OMVs in sterile filtered culture samples directly. NTA allows simultaneous size and concentration measurements of OMVs, and was modified in this chapter to process samples in high-throughput. 
Analysis of purified OMV quality is currently mainly performed based on protein content and composition, in combination with OMV size. The OMV component of $4 \mathrm{CMenB}$, for example, is dosed based on total protein content ( $25 \mu \mathrm{g} / \mathrm{dose})$ and the protein composition is assessed by SDS-PAGE [21, 22]. For a vaccine composition based on heterologous antigens expressed on OMVs, the amount of heterologous antigen is likely to be highly important for the efficacy of the vaccine. Determination of the amount of antigen can be performed by methods like ELISA or a protein composition measurement in combination with a total protein measurement. These methods result in an average concentration of antigen in the OMV product. However, the distribution of antigen over the OMVs is unknown and could be variable. Insight in this distribution could be generated by labeling of the antigen and subsequent measurement by for example gold-labelled electron microscopy. Alternatively, fluorescence NTA can be used where specific labeling of nanoparticles with highly fluorescent molecules as quantum dots give insight in the distribution of labelling over the particles, as has been shown for the fluorescent labeling of extracellular vesicles [23]. Moreover, high-resolution flow cytometry [24] and MALS [25] could be suited methods for these measurements.

OMV release per bacterium could be enhanced by sulfur depletion of Nm cultures. Chapter 4 showed that sulfate depletion induced a higher release of OMVs than cysteine depletion. The release of OMVs by sulfur source depletion was associated with oxidative stress responses. Oxidative stress could be applied directly on bacterial cultures by increasing the dissolved oxygen concentration as shown in Chapter 5. Applying high dissolved oxygen concentrations on a cysteine depleted culture resulted in a four-fold increase in OMV concentrations. We speculate that application of high dissolved oxygen tensions on a sulfate depleted culture would result in an even higher OMV yield. Although the exact mechanism of OMV formation was not found in this study, we observed that OMV release upon sulfate depletion was associated with enhanced phospholipid and LPS production, and thus follows the model of phospholipid accumulation based OMV release [26]. The combination of cysteine depletion and high dissolved oxygen tension resulted in the production of $90 \mathrm{mg}$ OMV associated protein per liter culture. Assuming a dose of $25 \mu \mathrm{g}$ OMV associated protein, this process results in 3600 human doses per liter culture. A production scale of 300L would suffice to produce 1 million doses per campaign. This yield is highly sufficient for clinical testing of an OMV-based vaccine concept. The scalability of a similar batch cultivation of $\mathrm{Nm}$ 
has been shown previously to be feasible up to 800L [27]. However, for this a low dissolved oxygen concentration was used [27]. High dissolved oxygen concentrations are a convenient parameter to use at production scale as this parameter is well controlled.

For a vaccine platform it is worthwhile to invest extra time and resources to optimize the production process in terms of yield and quality, since all new vaccines made with this platform will profit. Interestingly, the yields obtained in Chapter 6 were based on a low cell-density cultivation process, with a maximum cell density of 4 gram dry weight per liter culture (gdw/L). Other studies have already shown that it is possible to cultivate Nm up to high cell densities [28], reaching densities of $58 \mathrm{gdw} / \mathrm{L}$ [29]. These densities have been reached by applying a perfusion process that allows for simultaneous nutrient supply and removal of cell debris and inhibiting components. Another approach to improve OMV productivity is by implementing continuous production processes. Chapter 7 showed that continuous cultivation results in increased yearly productivities over batch-wise production. A 60-liter continuous bioreactor culture suffices to produce 1 million doses per week, which is a 5-fold reduction in size compared to the batch process, assuming comparable purification losses and one batch production per week. Future research is required to assess the feasibility of implementing fully continuous processing.

\section{Outlook}

There are several targets that could benefit from vaccine development through an OMVbased vaccine platform. A modular vaccine approach reduces the development time since a known production process can be used. Additionally, the known safety profile of the vaccine platform base could result in accelerated clinical trials. The OMV-based vaccine platform can form a solution for pathogens that are difficult to culture in lab conditions or pathogens that require high containment levels. The approach of this thesis is based on the expression of heterologous antigens by $\mathrm{Nm}$, and thus limited to microbial targets or other targets characterized by non-glycosylated protein antigens. Alternatively, the reach of the vaccine platform can be expanded to viral and therapeutic targets if the antigen is produced separately and coupled to the OMV. In this section, an outlook on future production of OMV-based vaccine is given and vaccine targets are discussed that could benefit from development through the Nm OMV platform. 


\section{Future production of OMVs}

An OMV-based vaccine platform production process can profit from a process based on disposables. Since production in disposables prevents cross contamination during production of multiple drug substances in a single factory, changeover procedures are minimal to produce multiple products in the same production facility. However, the production of microbials in disposable equipment is still limited by the aeration, cooling, and the size of disposable bioreactors [30,31]. Recently a single use fermenter was presented that is capable of growing high cell densities of Pichia pastoris up to 2000L scale [32]. In this reactor, sparging aeration was applied in combination with antifoam to prevent foam buildup. Antifoam in OMV production processes should be used with caution since antifoam is likely to be included in the membrane structure of OMVs [33]. Therefore, mechanical foam disruption would be preferred over antifoaming agents, but this is currently unavailable for disposable bioreactors. To benefit from production in disposables, antifoaming agents approved as excipient could be used. Interestingly, the drug Simethicone has been based on an antifoaming agent to relieve pain and discomfort caused by excess gas in the intestine and stomach $[34,35]$. This drug is approved for oral use, but also included in the intramuscular delivered antibiotic drug Retarpen $®$ (Sandoz). Simethicone is sold as antifoaming agent as excipient grade and could therefore be used as antifoaming agent during the production of OMVs, although future research should elucidate the degree and effect of integration of antifoaming agent in OMVs. This antifoaming agent could thus be the basis to overcome aeration issues and allow for OMV production in disposable bioreactors.

\section{Broad coverage OMV-based Meningococcal disease vaccines}

The Nm OMV vaccine platform would be a good basis of a combinatorial meningococcal disease vaccine. Meningococcal disease in human is mainly caused by 6 serogroups of $N$. meningitidis $(\mathrm{A}, \mathrm{B}, \mathrm{C}, \mathrm{W}, \mathrm{X}, \mathrm{Y})$ that are classified based on their capsular polysaccharide $[36$, 37]. Currently there are vaccines on the market for either serogroup B meningococcal disease (Bexsero $®$, TrumenBa () and quadrivalent conjugate serogroup ACYW (Menveo $\AA$, Menactra $\AA$ ). At this time there is no serogroup X meningococcal vaccine available. However, a pentavalent conjugate vaccine for serogroup ACYWX has been designed and was shown to be well-tolerated in a phase I clinical trial [38]. 
N. meningitidis is a highly variable bacterium [39]. Antigenic outer membrane proteins will undergo antigenic shift or even gene deletion [40]. This can cause reduced efficacy of current serogroup B vaccines, which are based on outer membrane proteins. Moreover, serogroup replacement has been observed, although not associated with vaccine introduction $[41,42]$. Additionally, unencapsulated strains of $N$. meningitidis have been observed as the cause of multiple cases of invasive meningococcal disease [43]. Although rare, and possible associated with immunocompromised individuals, this does show that future protein based $\mathrm{Nm}$ vaccines can have advantages over capsule based conjugate vaccines. A possible solution to prevent antigenic shift is to generate OMVs lacking the highly variable major outer membrane porin PorA to induce immunity against more conserved surface antigens $[44,45]$. This approach was found to be safe, though less immunogenic than other vaccines [46]. Another approach, that was developed by the predecessors of Intravacc, is based on the expression of multiple variants of PorA on OMVs to induce protection against different Nm subtypes [47-49]. To improve this approach, a detergent-free extraction method has been developed to produce OMVs [1]. Interestingly, the use of sOMVs over eOMVs will result in an improved coverage against meningococcal disease as sOMVs showed immune responses against strains not included in the vaccine, indicating immune responses against other antigens than PorA, that were not observed in eOMV or dOMV groups [1]. Combination of the expression of multiple PorA variants and the use of Nm sOMVs could thus result in a broad protecting serogroup B meningococcal vaccine. Moreover, it would be possible to add polysaccharides to OMVs $[50,51]$. Capsular polysaccharides can be added by conjugation to OMVs, or by expression of the gene cluster for capsular polysaccharide synthesis in the OMV producing bacterium. By displaying capsular polysaccharides from serogroup ACWYX on serogroup B OMVs, a Pan (hexavalent) meningococcal vaccine composition could be created (Figure 3).

Taken together, the Nm OMV based vaccine platform could be the basis of new meningococcal vaccines. Such vaccines can provide a broad protection against different serogroups. Additionally, the use of spontaneous OMVs as vaccine basis could provide improved cross-protection over current vaccines. The development of a combinatorial meningococcal disease vaccine based on Nm OMVs is therefore promising. 

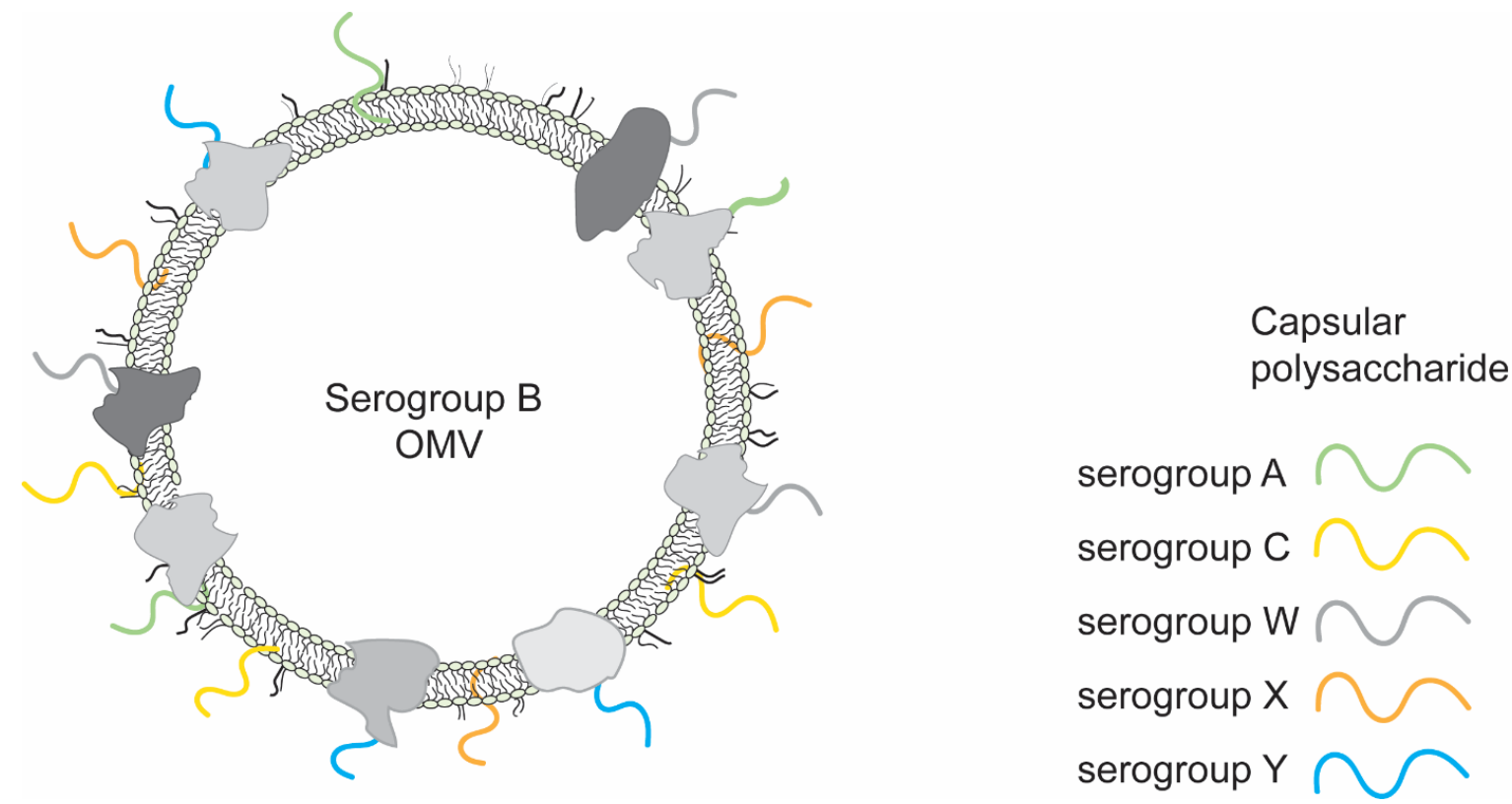

Figure 3. Multivalent meningococcal vaccine approach. The serogroup B N. meningitidis OMV can be used as carrier for capsular polysaccharides. Capsular polysaccharides from other serogroups could be either expressed on the OMV or produced separately followed by conjugation to the OMV vaccine base, to create a combinatorial meningococcal disease vaccine.

\section{OMV-based Lyme disease vaccine}

The production of heterologous antigens on Nm OMVs was assessed by introducing Lyme disease antigens as described in Chapter 6. The development of a Lyme disease (Lyme borreliosis) vaccine may thus be the most straightforward vaccine from the data presented in this thesis. A Lyme disease vaccine is highly desired since the incidence of Lyme disease continuously increases [52]. Lyme disease is caused by Borrelia burgdorferi sensu lato, that is transmitted to humans during the blood feeding of ticks $[53,54]$. No herd immunity can be obtained since B. burgdorferi is maintained in cycles between ticks and small mammal reservoirs [55]. While antibiotic treatment during early infection is often successful, diagnosis of infection is difficult and if untreated the localized infection can cause serious systemic disease. A vaccine was available (LYMErix) and effective, but was withdrawn due to inadequate market results in 2002, which was partially caused by the uncertainty about the vaccine-induced immunity period. Based on this, booster vaccination was required as often as once per year [56]. To date, no vaccine is available, while there is a need for a new Lyme disease vaccine [57]. 
LYMErix was based on recombinant lipidated surface protein OspA of B. burgdorferi adjuvated with aluminum hydroxide [58]. Concerns have been raised on possible autoimmunity due to the homology between OspA residues 165-173 with human leukocyte function-associated antigen 1 [59]. However, increased risk of arthritis or other adverse effects were not observed [60]. Modified OspA molecules have been generated lacking OspA residues 165-173, and thus eliminating the possibility of autoimmunity [61]. In Chapter 6, we expressed OspA on spontaneous released Nm OMVs. The induction of specific antibodies was shown previously for OspA expressed in a similar manner on the surface of detergent-free extracted OMVs [62]. The surface localization of antigens is likely required to improve the immunogenicity [63], as has been shown for whole-cells $[64,65]$. Immunogenicity experiments should be done to evaluate the evoked immune responses by the sOMVs produced in Chapter 6. An important question is whether there are differences in the immune response against the heterologous antigen expressed on eOMV or sOMV. Besides the immunogenicity, the location of expression needs to be characterized in more detail. This could be measured by antibody-labeled electron microscopy to elucidate the heterogeneity of antigens expressed on OMVs. The density of heterologous antigen is likely an important parameter for the efficacy of the vaccine. The expression of increased densities of heterologous peptides on virus-like particles, for example, showed that increasing densities enhanced the peptide specific immune response [66]. More research on the heterologous antigen concentration on OMVs is required to determine the design space of heterologous antigens expressed Nm OMVs as vaccine platform.

It remains questionable whether OspA alone would be sufficient for a new Lyme disease. OspA is expressed in the midgut of the tick and severely downregulated upon feeding of the tick. Anti OspA antibodies would thus inhibit transmission by targeting spirochetes in ticks. To obtain targeting of spirochetes after infection, other antigens must be included in future vaccine compositions. Many other antigens than OspA have been found, although most are highly variable [67]. Antibodies targeting outer protein $C$ (OspC), which is expressed in mammals, results in antibody mediated immunity [68].

Expression of B. burgdorferi antigens on Nm OMVs allows straightforward expression of both OspA and other antigens like OspC on a single OMV. Additionally, multiple OspA and OspC types could be presented on this platform. Furthermore, the Nm OMV will 
result in a balanced immune response, whereas more and more studies suggest a role for T-cell mediated immunity in the prevention of Lyme disease. Overall, the approach of expressing B. burgdorferi antigens on Nm OMVs shows potential as future Lyme disease vaccine.

\section{Nm OMV based vaccine platform for low income countries}

OMV-based vaccines could have potential for use in low-income countries. OMVs can be the sole active ingredient of a vaccine composition as OMVs are self-adjuvating and provoke broad immune responses. The use of OMV-based vaccines in low income countries may be counterintuitive, as the price of the current OMV-based meningitis vaccine Bexsero $®$ is $102 \$$ or $97 €$ per dose $[69,70]$. This price even made the OMVbased vaccine not cost-effective for routine vaccination programs in high-income countries [71-74]. The high cost of OMV production has been posed as a drawback of OMV-based vaccines [75]. However, improved yields may reduce the cost-price of new OMV vaccines. Research on the biogenesis of OMVs has led to higher productivities. The volumetric yield of OMV production can be further enhanced by the implementation of continuous processing. Additionally, the production of OMVs released in the supernatant requires less unit-operations than extraction based production of OMVs.

Besides production costs, cold-chain transport and storage is a major hurdle in vaccine availability. Cold-chain transport regularly copes with accidental freezing of vaccines and their subsequent loss in potency [76]. The vesicle structure of OMVs protects the antigens from degradation by its intrinsic thermostability. Bordetella pertussis OMVs in solution show to be stable in antigenicity after incubation at $40^{\circ} \mathrm{C}$ for 30 days [77]. More harsh incubation of $\mathrm{Nm} \mathrm{sOMVs}$ at $100^{\circ} \mathrm{C}$ for 5 minutes did not rupture the vesicles, although it is unclear if in these extreme conditions the folding of antigens could be maintained (unpublished data). Incubation of B. pertussis OMVs at $65^{\circ} \mathrm{C}$ for 30 days did result in minor loss of immunogenicity [77]. The authors showed that spray drying results in maintained effective immunogenicity and no change in antigenicity after incubation at $65^{\circ} \mathrm{C}$ for 30 days. Detergent extracted Nm OMVs showed degradation of the OMV structure upon storage at $37^{\circ} \mathrm{C}$ or $56^{\circ} \mathrm{C}$ during 1 year [78]. Detergent extraction of OMVs is thus unfavorable for the stability of the vesicles. Vaccines based on OMVs could thus likely lack expensive cold-chain storage without compromising vaccine quality. 


\section{Nm OMV vaccine platform for rapid response to emerging infectious diseases}

In case of rapidly emerging infectious diseases, vaccines should be developed in a severely shortened time period. This can only be accomplished by using suitable vaccine platforms. The Nm OMV-based vaccine platform can be used for such rapid responses. For this application, an antigen needs to be identified and sequenced, before it can be expressed in the platform. For expression, the antigen sequence is codon optimized for expression in $\mathrm{Nm}$, linked to the surface-exposed expression system and synthesized by DNA-printing. Next, a clone expressing the heterologous antigen is generated. The expression of the antigen should be confirmed and production of OMVs can start. Lastly, release testing should ensure the newly generated OMVs have the desired characteristics. In the most optimal scenario, the production of a first batch of OMVvaccines starting from a novel antigen sequence expressed in $\mathrm{Nm}$ could be reached within 8 days (Figure 4). Likewise, it has been claimed by Abera Biosciences, that the design and manufacturing of an OMV-based vaccine decorated with surface antigens can be within 14-30 days [79]. Besides the promising potential of the vaccine platform in the scenario of disease outbreaks, this speed of production of new vaccine concepts can boost the development of new vaccines as the development trajectory can be shortened.

In this process, getting the antigen expressed in $\mathrm{Nm}$ is highly important. For the expression of antigens on $\mathrm{Nm}$ in this thesis, traditional molecular biology tools were applied. Nm is naturally competent for the uptake of DNA sequences [80, 81]. This uptake is characterized by a preference for self-DNA, that is forced by preferred uptake of DNA that contains a short sequence that is repetitively found in the Neisserial genome [82]. This DNA uptake sequence (DUS) can be used to artificially improve the uptake of DNA sequence that are not found in the genome of Neisserria spp. The use of antibiotic markers in this method is preferably avoided since generation of strains expressing multiple antigens require many different markers. A marker-free method has been described for the genomic engineering of $N$. gonorrhoeae [83], which can be used for Nm [84]. Novel CRISPR-Cas9 based methods could further speed-up the expression of heterologous antigens. Moreover, the introduction of multiple genes of interest in a single transformation becomes feasible by this method. Nm contains a Type-II CRISPR system [85] that can be used for genome editing [86], although the serogroup B isolate H44/76 that was used throughout this thesis does not encode an 
active CRISPR system. Since it is known that CRISPR interference blocks natural transformation [85], this may be the reason for natural competence of this isolate.

The rapid expression of new antigens on the surface of Nm OMVs is the basis of a Nm OMV-based vaccine platform that can be used in as rapid response during infectious outbreaks. Moreover, it is an important aspect in the development of other new vaccines, since a quick expression method allows for rapid screening of many antigens. The Nm OMV-based vaccine platform could have potential especially for microbial targets. Viral and therapeutic targets can only be addressed by separate OMV and antigen production as these targets likely require complex glycosylation that cannot be obtained by expression in $\mathrm{Nm}$. With the development of a rapid and markerless expression method a Nm OMV-based vaccine platform could be developed that supports rapid responses against emerging infectious microbial diseases.

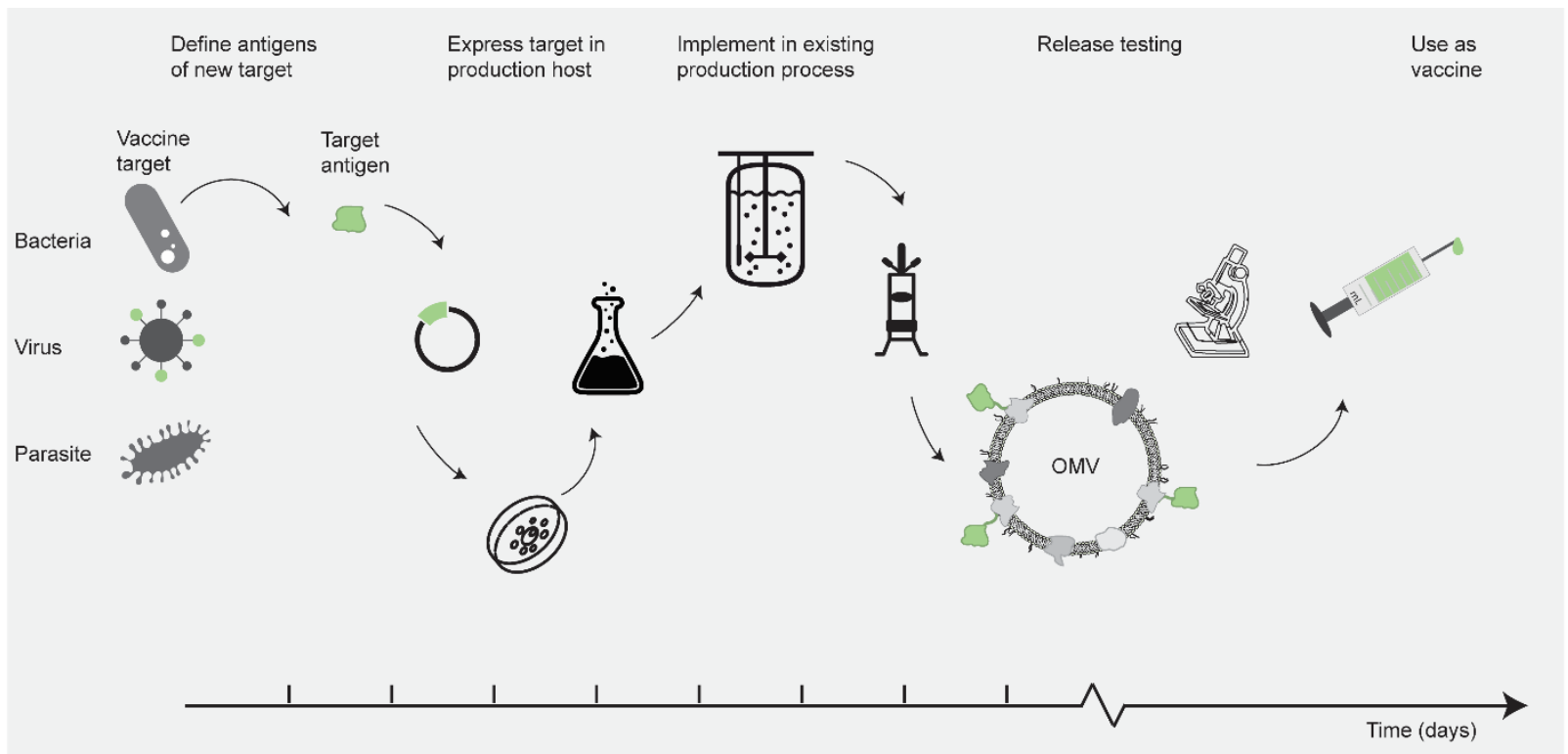

Figure 4. Nm OMV-based vaccine platform for rapid response against emerging infectious diseases.

After antigen selection, the sequence must be optimized for surface exposed expression in Nm. Clones expressing the antigen of interest are selected and used for OMV production. Starting from a shaker flask culture, a production culture is grown under OMV stimulating conditions, and OMVs containing the heterologous antigen are purified. After release testing and formulation, the OMVs can be directly used as vaccines.

\section{Conclusion: OMVs have potential as a modular vaccine platform}

The results of the studies in this thesis show that OMVs can be produced in large amounts based on scalable unit operations, which was reached by optimizing the amount of OMVs released by the bacteria in the culture supernatant. Heterologous 
antigens can be expressed on these OMVs, and the platform shows many interesting possibilities for the development of vaccines based on an Nm OMV-based vaccine platform. Increased knowledge on the release of OMVs can result in further optimization of OMV production processes and result in increased OMV yields and a further reduction in costs. Future studies should address the immune response against heterologous antigens and against the OMV-platform itself upon repetitive use. An OMVbased vaccine platform can provide a cost-price reduction in OMV production which could make OMV-based vaccines feasible for low-income countries. The development of OMV-based vaccines is highly suitable for antimicrobial resistant pathogens, for which vaccines are quickly needed. The development of vaccines for Lyme disease and serogroup B meningococcal disease based on Nm OMVs is possible in the near future and would provide an actual improvement for human health.

\section{References}

1. van de Waterbeemd, B., et al., Improved OMV vaccine against Neisseria meningitidis using genetically engineered strains and a detergent-free purification process. Vaccine, 2010. 28(30): p. 4810-6.

2. Christian, K.A., et al., What We Are Watching - Top Global Infectious Disease Threats, 2013-2016: An Update from CDC's Global Disease Detection Operations Center. Health Security, 2017. 15(5): p. 453462.

3. Knol, M.J., et al., Temporal associations between national outbreaks of meningococcal serogroup $\mathrm{W}$ and $\mathrm{C}$ disease in the Netherlands and England: an observational cohort study. The Lancet Public Health, 2017. 2(10): p. e473-e482.

4. Knol, M.J., et al., Implementation of MenACWY vaccination because of ongoing increase in serogroup W invasive meningococcal disease, the Netherlands, 2018. Eurosurveillance, 2018. 23(16): p. 18-00158.

5. Jansen, K.U., C. Knirsch, and A.S. Anderson, The role of vaccines in preventing bacterial antimicrobial resistance. Nature Medicine, 2018. 24: p. 10.

6. Nikaido, H., Multidrug Resistance in Bacteria. Annual review of biochemistry, 2009. 78: p. 119-146.

7. Mishra, R.P.N., et al., Vaccines and antibiotic resistance. Current Opinion in Microbiology, 2012. 15(5): p. 596-602.

8. Charlton Hume, H.K. and L.H.L. Lua, Platform technologies for modern vaccine manufacturing. Vaccine, 2017. 35(35, Part A): p. 4480-4485.

9. Kulp, A. and M.J. Kuehn, Biological Functions and Biogenesis of Secreted Bacterial Outer Membrane Vesicles. Annual Review of Microbiology, 2010. 64(1): p. 163-184.

10. Bjune, G., et al., Effect of outer membrane vesicle vaccine against group B meningococcal disease in Norway. The Lancet, 1991. 338(8775): p. 1093-1096.

11. Sierra, G.V., et al., Vaccine against group B Neisseria meningitidis: protection trial and mass vaccination results in Cuba. NIPH annals, 1991. 14(2): p. 195-207; discussion 208-10.

12. Cassio de Moraes, J., et al., Protective efficacy of a serogroup B meningococcal vaccine in Sao Paulo, Brazil. The Lancet, 1992. 340(8827): p. 1074-1078.

13. Thornton, V., et al., Safety and immunogenicity of New Zealand strain meningococcal serogroup B OMV vaccine in healthy adults: Beginning of epidemic control. Vaccine, 2006. 24(9): p. 1395-1400.

14. Boslego, J., et al., Efficacy, safety, and immunogenicity of a meningococcal group B (15:P1.3) outer membrane protein vaccine in Iquique, Chile. Vaccine, 1995. 13(9): p. 821-829.

15. Holst, J., et al., Properties and clinical performance of vaccines containing outer membrane vesicles from Neisseria meningitidis. Vaccine, 2009. 27: p. B3-B12.

16. Zariri, A., et al., Modulating endotoxin activity by combinatorial bioengineering of meningococcal lipopolysaccharide. Scientific Reports, 2016. 6: p. 36575. 
17. Keiser, P.B., et al., A phase 1 study of a meningococcal native outer membrane vesicle vaccine made from a group B strain with deleted lpxL1 and synX, over-expressed factor H binding protein, two PorAs and stabilized OpcA expression. Vaccine, 2011. 29(7): p. 1413-1420.

18. Mug-Opstelten, D. and B. Witholt, Preferential release of new outer membrane fragments by exponentially growing Escherichia coli. Biochimica et Biophysica Acta (BBA) - Biomembranes, 1978. 508(2): p. 287-295.

19. Beveridge, T.J., Structures of Gram-negative cell walls and their derived membrane vesicles. Journal of Bacteriology, 1999. 181(16): p. 4725-4733.

20. De Benedetto, G., et al., Multiple Techniques for Size Determination of Generalized Modules for Membrane Antigens from Salmonella typhimurium and Salmonella enteritidis. ACS Omega, 2017. 2(11): p. 8282-8289.

21. Serruto, D., et al., The new multicomponent vaccine against meningococcal serogroup B, 4CMenB: Immunological, functional and structural characterization of the antigens. Vaccine, 2012. 30: p. B87B97.

22. European_Medicines_Agency_Committee_for_Medicinal_Products_for_Human_Use. Assessment report: Bexsero, meningococcal group B vaccine. Nov 15, 2012 Oct 28, 2018]; Available from: https://www.ema.europa.eu/documents/assessment-report/bexsero-epar-public-assessmentreport_en.pdf.

23. Dragovic, R.A., et al., Sizing and phenotyping of cellular vesicles using Nanoparticle Tracking Analysis. Nanomedicine: Nanotechnology, Biology and Medicine, 2011. 7(6): p. 780-788.

24. Welsh, J.A., et al., Extracellular Vesicle Flow Cytometry Analysis and Standardization. Frontiers in Cell and Developmental Biology, 2017. 5(78).

25. Zhang, H., et al., Identification of distinct nanoparticles and subsets of extracellular vesicles by asymmetric flow field-flow fractionation. Nature Cell Biology, 2018. 20(3): p. 332-343.

26. Volgers, C., P.H.M. Savelkoul, and F.R.M. Stassen, Gram-negative bacterial membrane vesicle release in response to the host-environment: different threats, same trick? Critical Reviews in Microbiology, 2018. 44(3): p. 258-273.

27. Baart, G.J.E., et al., Scale-up for bulk production of vaccine against meningococcal disease. Vaccine, 2007. 25(34): p. 6399-6408.

28. Gerke, C., et al., Production of a Shigella sonnei vaccine based on generalized modules for membrane antigens (GMMA), 1790GAHB. PloS one, 2015. 10(8): p. e0134478.

29. Dehottay, P.M.H., et al., Fermentation of fastidious bacterial strain in perfusion suspension culture. 2014, GLAXOSMITHKLINE BIOLOGICALS S.A.

30. Shukla, A.A. and U. Gottschalk, Single-use disposable technologies for biopharmaceutical manufacturing. Trends in Biotechnology, 2013. 31(3): p. 147-154.

31. Jossen, V., et al., 7 - Stirred Bioreactors: Current State and Developments, With Special Emphasis on Biopharmaceutical Production Processes, in Current Developments in Biotechnology and Bioengineering, C. Larroche, et al., Editors. 2017, Elsevier. p. 179-215.

32. Mariner, J.C., et al., Rinderpest Eradication: Appropriate Technology and Social Innovations. Science, 2012. 337(6100): p. 1309-1312.

33. van der Pol, L., M. Stork, and P. van der Ley, Outer membrane vesicles as platform vaccine technology. Biotechnology Journal, 2015. 10(11): p. 1689-1706.

34. Valentine, W. and W.K. Valentine, Simethicone/calcium silicate composition. 1990, Google Patents.

35. Metcalf, T.J., et al., Simethicone in the Treatment of Infant Colic: A Randomized, Placebo-Controlled, Multicenter Trial. Pediatrics, 1994. 94(1): p. 29-34.

36. Halperin, S.A., et al., The changing and dynamic epidemiology of meningococcal disease. Vaccine, 2012. 30: p. B26-B36.

37. Rosenstein, N.E., et al., Meningococcal Disease. New England Journal of Medicine, 2001. 344(18): p. 1378-1388.

38. Chen, W.H., et al., Safety and immunogenicity of a pentavalent meningococcal conjugate vaccine containing serogroups A, C, Y, W, and X in healthy adults: a phase 1, single-centre, double-blind, randomised, controlled study. The Lancet Infectious Diseases.

39. Meyer, T.F. and S.A. Hill, 8 - Genetic Variation in the Pathogenic Neisseria Species, in Antigenic Variation, A. Craig and A. Scherf, Editors. 2003, Academic Press: London. p. 142-164.

40. Harrison, L.H., et al., Antigenic Shift and Increased Incidence of Meningococcal Disease. The Journal of Infectious Diseases, 2006. 193(9): p. 1266-1274.

41. Wang, X., et al., Changes in the Population Structure of Invasive Neisseria meningitidis in the United States After Quadrivalent Meningococcal Conjugate Vaccine Licensure. The Journal of Infectious Diseases, 2015. 211(12): p. 1887-1894. 
42. Zhu, B., et al., Prevalence and genetic characteristics of 4CMenB and rLP2086 vaccine candidates among Neisseria meningitidis serogroup B strains, China. Vaccine, 2018. 36(15): p. 1983-1989.

43. Ganesh, K., et al., Molecular characterization of invasive capsule null Neisseria meningitidis in South Africa. BMC Microbiology, 2017. 17(1): p. 40.

44. Weynants, V.E., et al., Additive and Synergistic Bactericidal Activity of Antibodies Directed against Minor Outer Membrane Proteins of Neisseria meningitidis. Infection and Immunity, 2007. 75(11): p. 5434-5442.

45. Shea, M.W., The Long Road to an Effective Vaccine for Meningococcus Group B (MenB). Annals of Medicine and Surgery, 2013. 2(2): p. 53-56.

46. Bonvehí, P., et al., Three Doses of an Experimental Detoxified L3-Derived Lipooligosaccharide Meningococcal Vaccine Offer Good Safety but Low Immunogenicity in Healthy Young Adults. Clinical and Vaccine Immunology : CVI, 2010. 17(9): p. 1460-1466.

47. Claassen, I., et al., Production, characterization and control of a Neisseria meningitidis hexavalent class 1 outer membrane protein containing vesicle vaccine. Vaccine, 1996. 14(10): p. 1001-1008.

48. van der Ley, P., J. van der Biezen, and J.T. Poolman, Construction of Neisseria meningitidis strains carrying multiple chromosomal copies of the porA gene for use in the production of a multivalent outer membrane vesicle vaccine. Vaccine, 1995. 13(4): p. 401-407.

49. van den Dobbelsteen, G.P.J.M., et al., Immunogenicity of a combination vaccine containing pneumococcal conjugates and meningococcal PorA OMVs. Vaccine, 2007. 25(13): p. 2491-2496.

50. Chen, L., et al., Outer membrane vesicles displaying engineered glycotopes elicit protective antibodies. Proceedings of the National Academy of Sciences, 2016. 113(26): p. E3609-E3618.

51. Price, N.L., et al., Glycoengineered Outer Membrane Vesicles: A Novel Platform for Bacterial Vaccines. Scientific Reports, 2016. 6: p. 24931.

52. Sykes, R.A. and P. Makiello, An estimate of Lyme borreliosis incidence in Western Europedagger. J Public Health (Oxf), 2017. 39(1): p. 74-81.

53. Johnson, R.C., et al., Borrelia burgdorferi sp. nov.: Etiologic Agent of Lyme Disease. International Journal of Systematic and Evolutionary Microbiology, 1984. 34(4): p. 496-497.

54. Stanek, G., et al., Lyme borreliosis. The Lancet, 2012. 379(9814): p. 461-473.

55. Kamradt, T., Lyme disease and current aspects of immunization. Arthritis Research \& Therapy, 2001. 4(1): p. 20.

56. Nigrovic, L E. and K M. Thompson, The Lyme vaccine: a cautionary tale. Epidemiology and Infection, 2007. 135(1): p. 1-8.

57. Plotkin, S.A., Correcting a public health fiasco: The need for a new vaccine against lyme disease. Clinical Infectious Diseases, 2011. 52(SUPPL. 3): p. S271-S275.

58. Steere, A.C., et al., Vaccination against Lyme Disease with Recombinant Borrelia burgdorferi OuterSurface Lipoprotein A with Adjuvant. New England Journal of Medicine, 1998. 339(4): p. 209-215.

59. Gross, D.M., et al., Identification of LFA-1 as a Candidate Autoantigen in Treatment-Resistant Lyme Arthritis. Science, 1998. 281(5377): p. 703-706.

60. Ball, R., et al., HLA type and immune response to Borrelia burgdorferi outer surface protein a in people in whom arthritis developed after Lyme disease vaccination. Arthritis \& Rheumatism, 2009. 60(4): p. 1179-1186.

61. Izac, J.R., et al., Identification of a defined linear epitope in the OspA protein of the Lyme disease spirochetes that elicits bactericidal antibody responses: Implications for vaccine development. Vaccine, 2017. 35(24): p. 3178-3185.

62. Salverda, M.L.M., et al., Surface display of a borrelial lipoprotein on meningococcal outer membrane vesicles. Vaccine, 2016. 34(8): p. 1025-1033.

63. Daleke-Schermerhorn, M.H., et al., Decoration of outer membrane vesicles with multiple antigens by using an autotransporter approach. Applied and Environmental Microbiology, 2014. 80(18): p. 58545865.

64. Rizos, K., et al., Autodisplay: Efficacious Surface Exposure of Antigenic UreA Fragments from Helicobacter pylori in Salmonella Vaccine Strains. Infection and Immunity, 2003. 71(11): p. 6320-6328.

65. Lee, J.-S., et al., Surface-displayed viral antigens on Salmonella carrier vaccine. Nature Biotechnology, 2000. 18: p. 645.

66. Chuan, Y.P., et al., Effects of pre-existing anti-carrier immunity and antigenic element multiplicity on efficacy of a modular virus-like particle vaccine. Biotechnology and Bioengineering, 2013. 110(9): p. 2343-2351.

67. Poljak, A., et al., Identification and characterization of Borrelia antigens as potential vaccine candidates against Lyme borreliosis. Vaccine, 2012. 30(29): p. 4398-4406. 
68. Bockenstedt, L.K., et al., Borrelia burgdorferi strain-specific Osp C-mediated immunity in mice. Infection and Immunity, 1997. 65(11): p. 4661-4667.

69. CDC. The CDC Vaccine Price Lists. 2018 01-aug-2018 [cited 201808 Aug]; Available from: https://www.cdc.gov/vaccines/programs/vfc/awardees/vaccine-management/price-list/index.html.

70. Christensen, H., et al., Epidemiological impact and cost-effectiveness of universal vaccination with Bexsero ${ }^{\circledR}$ to reduce meningococcal group B disease in Germany. Vaccine, 2016. 34(29): p. 3412-3419.

71. Pouwels, K.B., et al., Cost-effectiveness of vaccination against meningococcal B among Dutch infants. Human Vaccines \& Immunotherapeutics, 2013. 9(5): p. 1129-1138.

72. Christensen, H., et al., Re-evaluating cost effectiveness of universal meningitis vaccination (Bexsero) in England: modelling study. BMJ : British Medical Journal, 2014. 349.

73. Christensen, H. and C.L. Trotter, Modelling the cost-effectiveness of catch-up 'MenB' (Bexsero) vaccination in England. Vaccine, 2017. 35(2): p. 208-211.

74. Tirani, M., M. Meregaglia, and A. Melegaro, Health and Economic Outcomes of Introducing the New MenB Vaccine (Bexsero) into the Italian Routine Infant Immunisation Programme. PLOS ONE, 2015. 10(4): p. e0123383.

75. Tan, K., et al., Outer Membrane Vesicles: Current Status and Future Direction of These Novel Vaccine Adjuvants. Frontiers in Microbiology, 2018. 9: p. 783.

76. Matthias, D.M., et al., Freezing temperatures in the vaccine cold chain: A systematic literature review. Vaccine, 2007. 25(20): p. 3980-3986.

77. Kanojia, G., et al., Development of a thermostable spray dried outer membrane vesicle pertussis vaccine for pulmonary immunization. Journal of Controlled Release, 2018. 286: p. 167-178.

78. Arigita, C., et al., Stability of mono- and trivalent meningococcal outer membrane vesicle vaccines. Vaccine, 2004. 22(5): p. 629-642.

79. AberaBiosciences. Fast production at low cost. 2018 [cited 2018 2018-09-25]; Available from: aberabio.com/technology.

80. Catlin, B.W., Transformation of Neisseria meningitidis by deoxiribonucleates from cells and from culture slime. Journal of Bacteriology, 1960. 79(4): p. 579-590.

81. Hamilton, H.L. and J.P. Dillard, Natural transformation of Neisseria gonorrhoeae: from DNA donation to homologous recombination. Molecular Microbiology, 2005. 59(2): p. 376-385.

82. Obergfell, K.P. and H.S. Seifert, Mobile DNA in the pathogenic Neisseria. Microbiology spectrum, 2015. 3(3): p. MDNA3-0015-2014.

83. Johnston, D.M. and J.G. Cannon, Construction of mutant strains of Neisseria gonorrhoeae lacking new antibiotic resistance markers using a two gene cassette with positive and negative selection. Gene, 1999. 236(1): p. 179-184.

84. Richardson, A.R. and I. Stojiljkovic, Mismatch repair and the regulation of phase variation in Neisseria meningitidis. Molecular Microbiology, 2001. 40(3): p. 645-655.

85. Zhang, Y., et al., Processing-Independent CRISPR RNAs Limit Natural Transformation in Neisseria meningitidis. Molecular cell, 2013. 50(4): p. 488-503.

86. Hou, Z., et al., Efficient genome engineering in human pluripotent stem cells using Cas 9 from Neisseria meningitidis. Proceedings of the National Academy of Sciences, 2013. 110(39): p. 15644. 
Addendum

Summary

Dankwoord

Curriculum vitae

List of publications

Overview of completed training activities 


\section{Summary}

Development of new vaccines based on vaccine platforms forms an interesting opportunity to significantly reduce the development time. New vaccines are required to keep up with newly emerging diseases that spread quickly in the interconnected global world. The traditional development of new vaccines is a lengthy process, as a new production process must be developed for each vaccine. A vaccine platform allows the use of the existing production process for new vaccine targets. Bacterial outer membrane vesicles (OMVs) produced by Neisseria meningitidis are highly suitable candidates to form a vaccine platform. $N$. meningitidis OMVs have been safely used as meningococcal vaccines. OMVs are non-replicative nanoparticles derived from the bacterial membrane, that can display heterologous antigens. $N$. meningitidis OMVs have been produced by extraction of vesicle like structures from bacterial cells. However, OMVs spontaneously released from bacteria have advantages over extracted OMVs as they can be directly purified from the supernatant of the bacterial culture, have enhanced quality, and trigger broader immune responses. On the downside, the yields of spontaneously released OMVs are low. The aim of this thesis was to obtain a better understanding of outer membrane vesicle formation by Neisseria meningitidis and OMV quality, and use this to develop improved OMV production processes that can become a cost-effective basis for an OMV-based vaccine platform

A vaccine platform should be versatile and adaptable for the addition of heterologous antigens onto the OMV. In Chapter 2 we explored existing methods for antigen decoration of OMVs through a comprehensive literature review. We distinguished two approaches of OMV platforms, based on either separate production of antigen and OMV followed by coupling or production of the antigen directly by the OMV producing bacterium. Separate antigen production seems more suitable for viral and therapeutic targets as it allows coupling of complex glycosylated targets to the OMV. Production of antigens directly by the OMV producing bacterium is probably more suited for microbial targets and allows for the most straightforward production process.

To optimize OMV production processes, a new method for OMV quantification was needed as current quantification methods of OMVs were indirect and elaborate. In Chapter 3 we successfully used nanoparticle tracking analysis to quantify OMVs directly from sterile filtered culture samples, in a high-throughput manner. Now that we had a more reliable method available to quantify OMV release, our next step was to improve the OMV yields by studying the release of OMVs from the bacterium. A previous study had shown that cysteine depletion causes a stationary growth phase in which OMVs are released. In Chapter 4 we show that sulfur depletion in general resulted in OMV release of $N$. meningitidis cultures and found that sulfate depltion results in an even higher level of OMV release. Mechanistically, OMVs were enriched in phospholipids following sulfate depletion, suggesting that enrichment of phospholipids is an important factor in the OMV release process. 
A second parameter is oxidative stress that had been previously observed in cysteine depleted cultures, as well as in the sulfate depleted cultures described in Chapter 4 . We found that high dissolved oxygen tension could mimick this situation and trigger increased OMV release. Because dissolved oxygen tension is a well-controlled process parameter, high dissolved oxygen concentrations could be conveniently used to stimulate OMV release. This was demonstrated in Chapter 5 where we showed that sulfur depletion and high dissolved oxygen tension stimulate the OMV release per bacterium and can be applied in batch production processes.

Chapter 6 presents a proof of concept of the OMV-based vaccine platform in which the findings from the previous chapters were combined. We expressed outer surface protein A and outer surface protein C of Borrelia burgdorferi, the cause of Lyme disease, on $N$. meningitidis OMVs. These OMVs with heterologous model antigens were produced in a batch production process. In this process, sulfur depletion and high-dissolved oxygen concentrations were combined to establish high OMV yields. Purification based on scalable unit-operations resulted in a recovery of $90 \mathrm{mg}$ OMV associated protein per liter culture. This production proces could be used as a basis for the development of novel Lyme disease vaccines. Lastly, in chapter 7 we suggest that OMV production can be further improved by adopting continuous production processes. Continuous production results in increased volumetric productivities, enhanced process control, and reduced variability. However, before continuous OMV production can be used for OMV vaccines, a method to assure OMV quality in the lengthy cultivations needs to be developped.

This thesis shows that high yields of spontaneously released $N$. meningitidis OMVs can be obtained by stimulating release of OMVs from the bacterium by process parameters. These OMVs show potential as modular production platform and could boost future vaccine development. OMV based vaccine platforms will reduce the time required to develop new vaccines, which is urgently needed to meet the demand for new vaccines. 


\section{Dankwoord}

Vlak voordat dit boekje naar de drukker gaat, voelt het als een bijzonder moment om iedereen te bedanken die een bijdrage heeft geleverd aan dit proefschrift. In de maanden vakantie die tussen het schrijven van de hoofdstukken en het printen van dit boekje zitten heb ik veel aan jullie gedacht.

Als eerste wil ik uiteraard mijn promotieteam bedanken. Michiel, wat heb je veel tijd vrij gemaakt om dit project te begeleiden. Het was erg fijn dat de deur van je kantoor altijd open stond. Bedankt voor de vrijheid om mijn ideeën in dit project volledig de vrije loop te laten. René, bedankt voor het hartelijke welkom in jouw groep in Wageningen. Op de terugweg van onze overleggen heb ik eigenlijk elke keer teruggedacht aan jouw messcherpe samenvattingen, die elke keer begonnen met: "dus eigenlijk bedoel je..." Dirk, het was erg fijn dat je de tijd nam om zo vaak in Bilthoven te overleggen. Jouw betrokkenheid bij dit project heeft veel bijgedragen aan het onderzoek. Ik heb erg veel geleerd van jouw eerlijke en kritische blik op de proceskunde en de wetenschap. Ook heb je samen met René erg geholpen om mijn soms ietwat ambitieuze planning om te zetten in een realistische.

Bacteriële PD-afdeling: Wat is het een leuke groep om in te werken! Jan, Ronald, Lonneke, Jikke, Shimaira, Jorike, Lilli, Carolien, Robert, Maarten, en natuurlijk ook Michiel. Jullie maken dat het ontwikkelen van een kwalitatief vaccinproductieproces voelt als een gezellige cursus bierbrouwen voor gevorderden. We mogen trots zijn op de megagrote labs en alle glimmende apparatuur die er staat. Ook op de iets sneller roestende toestellen. We kijken al lang uit naar de pizza's in Zeist. Gaan we dat snel plannen?

Bas, als mijn stagebegeleider in 2013 heb je een perfecte voorzet gegeven voor mijn $\mathrm{PhD}$ project. Ik heb nog vaak teruggekeken naar de experimenten die de basis zijn geweest van zo ongeveer elk hoofdstuk in dit boekje. Leo, als afdelingshoofd heb je mij de kans gegeven om verder met het OMV-project te gaan. Ik hoop dat jouw procesontwikkelings-ervaring in de toekomst kan helpen om het platform een fase verder te helpen. Daniëlle, ontzettend bedankt voor het oneindige vertrouwen en dat ik altijd bij je terecht kon voor advies! We moeten toch ons Achterhoeks wat vaker laten horen in Bilthoven. 
Kamergenoten! Sven en Martijn, wat was het gezellig om met jullie een luxe kantoor met mini-bar te delen. Misschien dat we met iets geknutsel aan het mobiele kantoorkoelsysteem zelfs nog wel climate control kunnen toevoegen. Merijn, bedankt voor fijne samenwerking op het Lyme project. Ik hoop dat we in de komende jaren kunnen aantonen hoe geweldig deze OMVs zijn! Joost, wat een fantastische inzichten heb jij zichtbaar kunnen maken met de mass-spec. Gideon en Diana, bedankt dat jullie een stapje extra doen en veel nuttige en opbouwende kritiek geven op de goed te keuren manuscripten. During my thesis project there were several interns who wrote a MSc thesis on OMVs. Wannisa, Kristian, Jasper, Fabian, Kevin, Natalia, and Lilli. Thanks so much for your hard work! I learned a lot from your fresh views on single-use bioreactors, continuous processing of OMVs and Cas9-based gene editing of Neisseria. Met de privatisering van Intravacc staan er een boel veranderingen op de planning. Met alle glimmende apparatuur en de recente verbouwingen zijn we er meer klaar voor dan ooit. En belangrijker dan alle randzaken zijn natuurlijk alle fijne collega's met bovendien een onschatbare waarde aan kennis en kunde. Dank jullie wel!

Achterhoekers! Geen inspanning zonder ontspanning! Zonder dat jullie het weten hebben jullie ook veel bijgedragen aan dit boekje. Niet alleen staan jullie altijd klaar (ik wist niet dat een verhuiswagen zo snel ingeladen en uitgeladen kon worden!), maar is het ook altijd gezellig. Waar we ook zijn. Ik ben benieuwd waar we dit keer met Pinksteren onze tenten op gaan gooien! Patrick \& Jesse Laten we nog vaak buiten de Achterhoek afspreken om wat te drinken. Ik hoop dat jullie inmiddels mijn hamburgerverslaving delen, want we hebben nog niet alle burgerrestaurants gehad! Lieve Mierenfamilie, wat hebben we samen een hoop meegemaakt de afgelopen jaren. Jullie steun is hartverwarmend. Lieve Jeanet, Brian, Bram \& Eva, wat is het altijd fijn om bij jullie langs te waaien. Eigenlijk komen we veel te weinig... Ook de familie van Whitney ben ik erg dankbaar. Mike, Manon, Cheryl, Lucy, Jesse \& Chrissy, wat is het leuk om jullie als (schoon)broers en zussen te hebben. Lieve Rob \& Monique, bedankt dat ik me zo snel welkom voelde bij jullie. Ik heb alweer zin in de volgende zomervakantie onder de zon en de vreetborden na het eten. Het wordt tijd dat die traditie overgenomen wordt op maandagavond in De Goejestraat. Lieve Daniëlla \& Harry, dank voor jullie uitgebreide interesse in mijn onderzoek. Ik kijk met plezier terug naar de 
vele gezellige momenten samen zoals de fantastische avonden met het vreemde woorden woordenboekspel.

Lieve pap, wat is het altijd fijn om weer bij jou thuis in Terborg te zijn. Nog geen seconde binnen en je hebt alweer een klus of project om te laten zien. Al maak ik me wel eens zorgen als je weer eens vrolijk vertelt dat er een boom is gesneuveld door de kettingzaag. Zelfs in de zware tijd toen mama ziek was bleef jij een grote steun en ontzettend zorgzaam. Het is zo fijn om te zien hoe gezellig je het nu samen met Alies hebt.

Whitney, wat was het een ontzettend leuke tijd om (grotendeels) tegelijkertijd aan onze promotieonderzoeken te werken! Uiteraard was het soms even afzien als we allebei weer eens lange dagen maakten, maar ik kijk toch met veel plezier terug naar de afgelopen jaren. Zonder jou had ik nooit zo veel van de wereld gezien. En wat was onze laatste reis gaaf! Dan de boerderij met alpacaweiland die we op een haar na gekocht hadden. Wie weet wat de toekomst brengt. We hebben het nu ook erg goed voor elkaar in ons gezellig huisje in Leiden. Ik heb ook ontzettend veel zin in toekomst die ons hier samen te wachten staan! Whit, je bent de allerliefste! 


\section{Curriculum vitae}

Thijs Gerritzen was born on January 1st, 1990 in Doetinchem, the Netherlands. He attended secondary school at St.-Ludgercollege in Doetinchem, from which he graduated in 2008. After obtaining his BSc degree in Biotechnology at Wageningen University (2011) he continued studying this field within the master Medical Biotechnology. In the second year of this MSc program he has written his master thesis within the department of microbiology of Wageningen University, under supervision of dr. Stan Brouns and dr. Peter Fineran. This project explored primed spacer acquisition of the E. coli CRISPR-Cas system

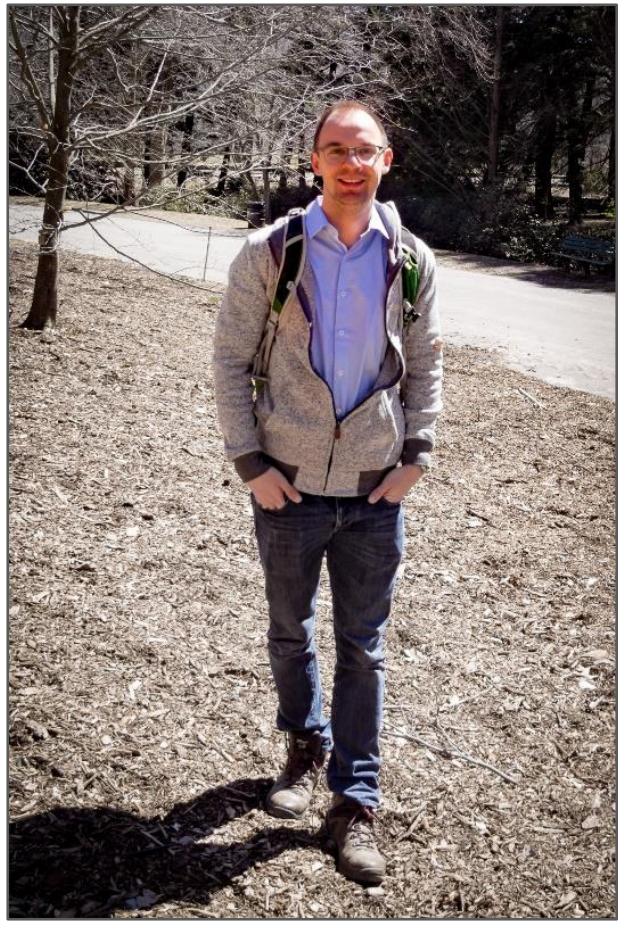
and resulted in an authorship on a publication. After this, he started an internship at the Institute for Translational Vaccinology (Intravacc), under supervision of dr. Bas van de Waterbeemd. During this internship he studied the possibilities of continuous outer membrane vesicle production. After obtaining his MSc degree in 2013 he started as junior scientist in the bacterial process development group of Intravacc. In 2016, the project on the production of outer membrane vesicles was continued by a $\mathrm{PhD}$ project in collaboration with the department of bioprocess engineering of Wageningen University and under supervision of prof. dr. René Wijffels, dr. Dirk Martens, and dr. Michiel Stork, which resulted in this thesis. During his $\mathrm{PhD}$ project he supervised several MSc students and presented his work at national and international conferences. After defending his thesis Thijs will continue working as scientist within the bacterial process development group of Intravacc. 


\section{List of publications}

Fineran, P.C., M.J.H. Gerritzen, M. Suarez-Diez, T. Kunne, J. Boekhorst, S.A. van Hijum, R.H. Staals and S.J. Brouns (2014). "Degenerate target sites mediate rapid primed CRISPR adaptation." Proc Natl Acad Sci U S A 111(16): E1629-1638.

Gerritzen, M.J.H., D.E. Martens, R.H. Wijffels and M. Stork (2017). "High throughput nanoparticle tracking analysis for monitoring outer membrane vesicle production." J Extracell Vesicles 6(1): 1333883.

Gerritzen, M.J.H., D.E. Martens, R.H. Wijffels, L. van der Pol and M. Stork (2017). "Bioengineering bacterial outer membrane vesicles as vaccine platform." Biotechnol Adv 35(5): 565-574.

Gerritzen, M.J.H., R.H.W. Maas, J. van den IJssel, L. van Keulen, D.E. Martens, R.H. Wijffels and M. Stork (2018). "High dissolved oxygen tension triggers outer membrane vesicle formation by Neisseria meningitidis." Microbial Cell Factories 17(1): 157.

Gerritzen, M.J.H., M.L.M. Salverda, D.E. Martens, R.H. Wijffels and M. Stork (2019). "Spontaneously released Neisseria meningitidis outer membrane vesicles as vaccine platform: Production and purification." Vaccine (accepted for publication)

Gerritzen, M.J.H., D.E. Martens, J.P. Uittenbogaard, R.H. Wijffels and M. Stork (2019). "Sulfate depletion triggers overproduction of phospholipids and the release of outer membrane vesicles by Neisseria meningitidis." Scientific Reports (accepted for publication)

Gerritzen, M.J.H., L. Stangowez. B. van de Waterbeemd, D.E. Martens, R.H. Wijffels and M. Stork. " Continuous production of Neisseria meningitidis outer membrane vesicles." (submitted for publication)

Gerritzen, M.J.H., van der pol, L.A., Stork, M. An improved process for producing outer membrane vesicles. European Patent Application Number EP17205138. 


\section{Overview of completed training activities}

\section{Discipline specific activities}

NBV meeting (KNCV, Wageningen)

Antiviral defense mechanisms (Wageningen university)

Dutch microbial biotechnology in action (KNVM, Utrecht)

NBV-PZ mini-symposium Vaccines (KNCV, Leiden)

Microbial Physiology and Fermentation Technology (BSDL, Delft)

Labchip basis training (Perkin Elmer, Bilthoven)

Nanoparticle Tracking Analysis (Malvern, Bilthoven)

NBV mini-symposium Continuous processing for therapeutic proteins (KNCV, Oss)

Applikon BioXpert training course (Applikon, Bilthoven)

Multifors training (Infors, Bilthoven)

International Society for Extracellular Vesicles meeting (ISEV, Rotterdam)

World of technology and science (WOTS, Utrecht)

Basics of extracellular vesicles (MOOC, ISEV)

Applikon Single-use technologies in upstream processing (Applikon, Delft)

BioProcess International European Summit (BPI, Amsterdam)

NBC-17 (KNCV, Wageningen)

NBV Workshop CRISPR/Cas (KNCV, Utrecht)

NBC-18 (KNCV, Wageningen)

Intravacc mini-Symposium (Intravacc, Bilthoven)

Vaccine technology VII (ECI, Mont Tremblant)

\section{General courses}

Tangential flow filtration (Spectrum Labs, Bilthoven)

Veilig werken met Biologische materialen (RIVM, Bilthoven)

Visie+ (GMP-X, Bilthoven)

GMP1 (GMP-X, Bilthoven)

Scientific writing (Wageningen Language Services, Wageningen)

Project Management Principles and Practices (MOOC, University of California)

Hands-on biological spill response (Intravacc, Bilthoven)

Spillkit training (course, Intravacc, Bilthoven)

Projectmatig werken (Schouten \& Nelissen, Bilthoven)

Six Sigma Yellow Belt (MOOC, University of Georgia)

\section{Optionals}

Preparation of research proposal

Monthly journal club

Seminars research department 

Intravacc (Institute for Translational Vaccinology) is a governmental institute for research and development activities in the field of translational vaccinology. All the research described in this thesis was funded by the Ministry of Health, Welfare and Sports (The Netherlands). The funder had no role in study design, data collection and analysis, or publishing decisions.

Printing of this thesis was financially supported by Wageningen University 


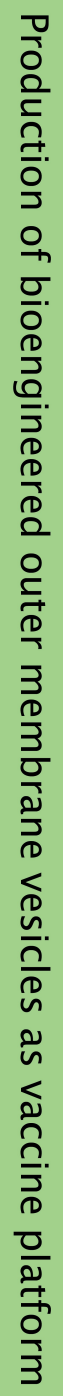

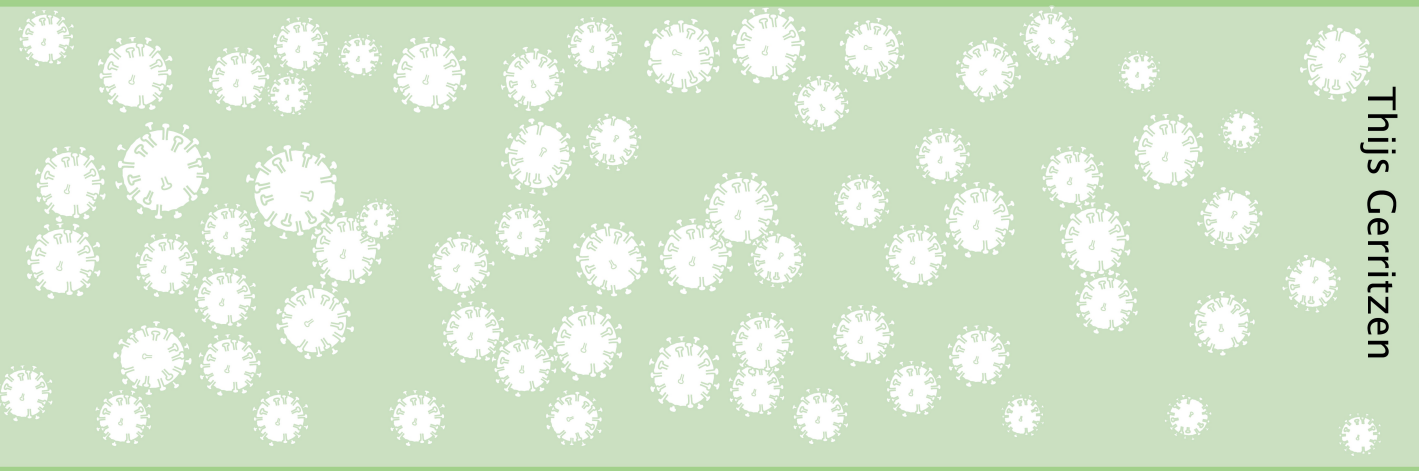

